COLUMBIA LIBRARIES OFFSITE

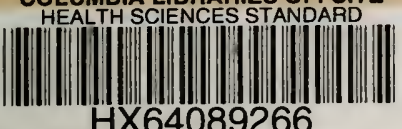

HX64089266

QP34 .P83 1906 An introduction to $p$

\title{
RECAP
}




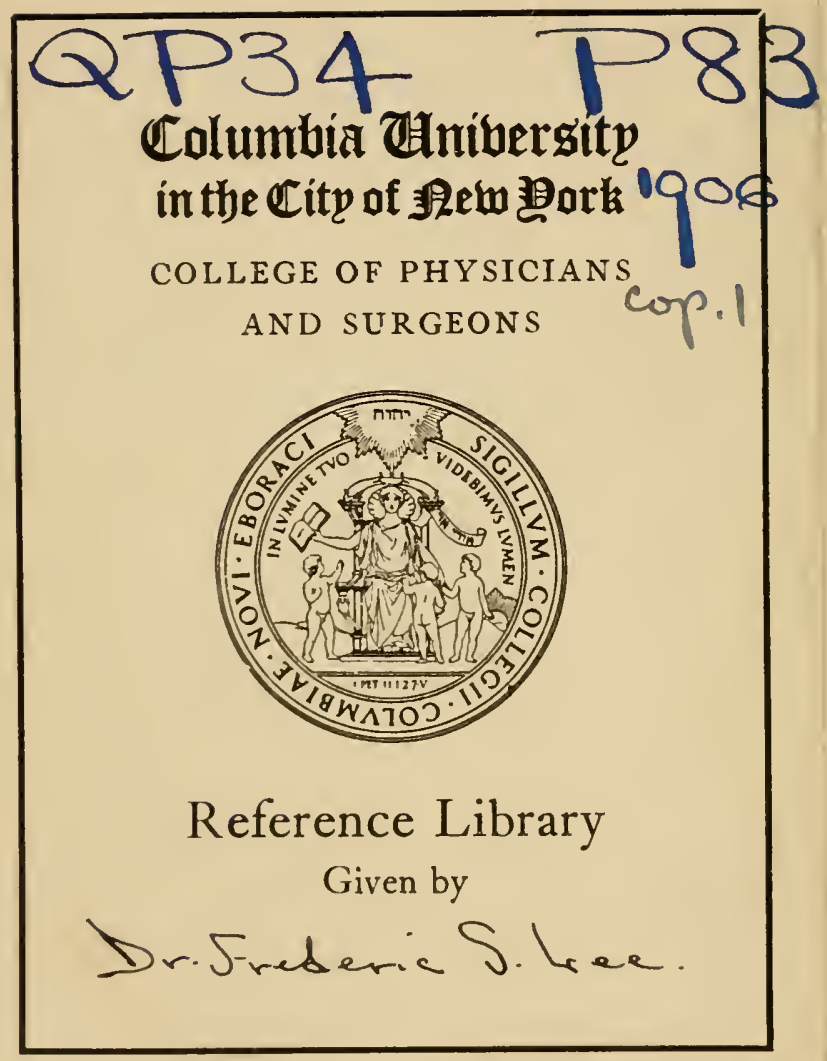


Thersinglece 

AN INTRODUCTION TO PHYSIOLOGY 



\title{
AN INTRODUC'TION
}

\author{
TO \\ P H Y S I O L G Y \\ BY
}

WILLIAM TOWNSEND PORTER, M.D.

ASSOCIATE PROFESSOR OF PHYSIOLOGY IN THE HARVARD MEDICAL SCHOOL

\section{THE UNIVERSITY PRESS}

Cambrioge, ftlass.

1906 
Copyright, 1906 ,

By W. T. Porter.

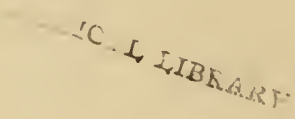

QP34

P8 3

1906

Uif 


\section{PREFACE TO THE SECOND EDITION}

Concentration, sequence, and election are fruitful principles in the higher education.

In 1898 the Committee on Medical Education, appointed by the Harvard Faculty of Medicine, reported in favor of the "concentration" system urged in the committee by the author in common with Professor W. T. Councilman. By this method, the first half-year in the Medical School is devoted to anatomy and histology, the second half-year to physiology and biological chemistry, the third half-year to pathology and bacteriology, and the fourth, fifth, and sixth half-years to practical medicine and surgery. Work under the new system began in the collegiate year of 1899-1900. In 1904, largely through the influence of Professor Bowditch, the seventh and eighth half-years were made elective, each student choosing for himself the studies best suited to his needs.

Concentration provides that the student shall not serve two masters, but shall study at one 
time only one principal subject, such as physiology or pathology, disciplines that do not yield readily to a divided mind. Sequence provides that a foundation shall be laid before the superstructure is attempted. Students now have an acquaintance with anatomy before they begin the study of physiology. Election somewhat tardily intrusts to university men rarely less than twenty-five years of age a. voice in the decision of their nearest affairs. The application of these principles to medical teaching has undoubtedly resulted in large savings of time and energy.

The economy of force secured by concentration and sequence has been highly valuable, though not indispensable, in the new teaching of physiology introduced by the author in February, 1900. The traditional teaching of physiology consists of lectures illustrated by occasional demonstrations and, in some instances, by experiments performed by the students themselves. The new method is fundamentally opposite. It consists of experiments and observations by the student himself. The didactic instruction, comprising lectures, written tests, recitations, conferences, and the writing and discussing of theses, follows the student's experiments and considers them in relation to the work of other observers. 
In the old method, the stress is upon the didactic teaching. In the new there is no less didactic teaching, but the stress is upon observation. The old method insensibly teaches men to rest upon authority, but the new directs them to nature.

The new method requires:

1. Printed accounts of the fundamental experiments and observations in physiology, taken from the original sources, and arranged in the most instructive sequence. The reference to the original source should be given in each case.

2. Accessory data grouped about the fundamental experiments. The accessory data should also be taken as directly as possible from the original sources, and the reference given in each case.

3. Apparatus of precision designed with the utmost simplicity upon lines that permit its manufacture in large quantities at small cost.

It is obvious that these conditions cannot be met without prolonged labor. Meanwhile, the annual classes in physiology must be taught. The present volume is a collection of fundamental and accessory experiments in several fields printed in an abbreviated form for the temporary use of Harvard Medical students and other interested persons. This collection is being completed 
viii PREFACE TO THE SECOND EDITION

and improved as rapidly as possible, and the data for the remaining fields are being brought together. In its final form this material will constitute "A Laboratory Text-book of Physiology."

The Harvard Medical School, January, 1906. 


\section{CONTENTS}

\section{PART I \\ THE GENERAL PROPERTIES OF LIVING TISSUES}

I

INTRODUCTION

Nerve-muscle preparation - Prelininary considerations regarding energy, stimulation, and irritability.

II

Methods of Electrical Stimulation

Introduction . . . . . . . . . . . . .

Kinetic theory - Osmotic pressure - Plasmolysis. Isotony - Estimation of osmotic pressure in the blood serum Surface tension - Electrolysis - Electrolytic solution pressure.

The Electhometer, the Rheochord, axd the Cell

Surface tension altered by electrical energy - Electrommeter - Rheochord - Long rheochord - Square rheochord - Simple key - Short-circuiting kej - Polarization - Pole-changer - Polarization current -- Dry cell.

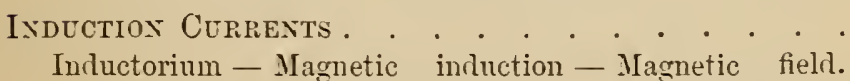
Lines of force-To produce electric induction, lines of magnetic force must be cut by circuit - Electromagnetic induction - On the construction of the inductorium-Enipirical graduation of inductorium - Platinum electrodes - Flat-jawed clamp - Round-jaweel 
clamp - Jouble clamp - Make and break induction currents as stimuli-Extra currents at opening and closing of primary current - Tetanizing currents - Induction in nerves - Exclusion of make or break current.

Unipolar IndUCtion . . . . . . . . • . .

\section{III}

The Graphic Method

The Graphic Method . . . . . . . . . .

Kymograph - Long paper kymograph - Light muscle lever - Writing lever - Tuning fork.

\section{IV}

The Electrical Stimulation of Muscle and Nerve

The Galuanic Current . . . . . . . 93

Non-polarizable electrodes - Moist chamber-Destruction of the brain by pithing - Paralysis of voluntary motion by curare - Opening and closing contraction - Clanges in intensity of stimnlus.

Polar Stimulation of Muscle . . . . . . . 101

Ureter - Gaskell Clamp - Intestine - Electro-magnetic signal-Tonic contraction - Physiological anode and cathole - Polar stimulation in heart.

Polar Strmulation of Nerve . . . . . . . . 113

Law of contraction - Changes in irritability - Changes in conductivity.

Stimulation of Human Nerves . . . . . . .

Stimulation of motor points - Polar stimulation of human nerves - Brass electrolles - Reaction of degeneration.

Galvanothopism . . . . . . . . . . . 137

Paramecium.

INFLULACE OF DURATton OF Strmulus . . . . . 138

I'onic contraction - Rhythmic contraction - Continuons galvanic stimulation of nerve may cause veriolic dis- 
Page

charge of nelve impulses - Polarization current - Polar fatigue - Opening and closing tetanus - Polar excitation in injured muscle.

Polar Ixhibition by the Galvanic Currext . . . 15.5

Heart - Polar inhibition in veratrinized muscle.

Stimulation Affected by the Form of the Muscle

Effect of the Axgle at Which the Currext Lines CUt THE Muscle Fibres . . . . . . . 157

The Ixduced Currest . . . . . . . . . 158

Chemical and Mechanical Stimulation

Chemical Stumulation

Effect of distilled water - Strong saline solutions - Drying - " Normal saline" - Importance of calcium Constant chemical stimulation may cause periodic contraction.

MLechaxical Stmulatiox . • . • . • • . . 166 Idio-muscular contraction.]

\section{VI}

\section{IrRITABILITY AND CONDUCTIVITY}

\section{IRRITABILITY AND CoNDUCTIVITY .}

Independent irritability of umuscle - Irritability and conductivity are separate properties of nerve - Minimal and maximal stimuli; threshold value - Summation of inadequate single stimuli - Relative excitability of flexor and extensor nerve fibres ; Ritter-Rollett phenomenonSpecific irritability of nerve greater than that of nuscle Irritability at different points of same nerve - Excitation wave remains in muscle or nerve fibre in which it starts - Same nerve fibre may conduct impulses both centripetally and centrifugally - Speed of nerve impulse. 


\section{PART II}

\section{THE INCOME OF ENERGY}

I

\section{Fermentation}

Hydrolysis of Starchi by Diastase

Conversion of starch to sugar by germinating barley - Conversion of starch to sugar by salivary diastase (ptyalin) Extraction of diastase from gelminating barley - Specific action of ferments.

Proteid Digestion by Pepsin

Gastric digestion of cooked beef and bread - Artificial gastric juice - Digestion with artificial gastric juiceExtraction of pepsin - Change of proteid to peptone by pepsin.

Splitting of Casein by Rennin

Rennin extract - Separation of remin - Precipitation of casein - Experiments of Arthus and Pagis.

Precipitation of Fiblin by Fibrin Fermest .

Buchanan's experinent - Extruction of fibrin ferment Extraction of fibrinogen - Precipitation of fibrinogen by fibrin fernent.

Ammoniacal, Felimentation of Uiea by Urease. .

Extraction of urease.

Sphiturix and Synthesis of Fats . . . . . .

Clocmistry of fats and soaps - Splitting of fats by the pancreatic juice - Preparation of neutral fat - The emulsion test for fatty acid - Extraction of lipase - IIycholysis of ethyl butyrate by lipase - Synthesis of neutral fat by lipase. 
IMMUNITY

Ehrlich's ricin experiments - Ricin antitoxine-Theory of immunity.

Haemolytic and Bacteriolytic Ferments . . .

Bordet's experiments.

Oxidizing FERMENTS

Schönbein's experiment-Further oxidations by animal tissues - Oxidation by nucleo-proteid - Oxidation about the nucleus - Glycolysis in blood - Oxidation not dependent on living cells of bloor - Relation of glycolysis to the pancreas and the lymph - Glycolytic ferment of pancreas.

Alcomolic Fermentation . . . . . . . . .

The yeast plant - Chemical relations of carbohydrates.

Activating Ferments . . . . . . . . . . .

Enterokinase - Conversion of trypsinogen to trypsin by - enterokinase.

\section{Absorption of Proteins}

Diffusion of proteids through dear membrane - Diffusion through living intestinal wall - Absorption velocity compared with diffusion velocity - Assimilable proteids Non-assimilable proteids - Alimentary albuminuria Albumose and peptone not ordinarily present in the blood or urine - Albumose and peptone changed in their passage through the intestinal wall.

absorption of Fats, Fat Acins, and Soaps . . .

Absorption of fat - Absorption of fat acids - Absorption of fat acid as a soap.

\section{LYMPH}

Permeability of vessel wall in inflanmation.

\section{BLOOD}

Specific Gravity . . . . . . . . . . . 264

Drawing the bloorl - Determination of specific gravity.

Counting the Corpuscles

Counting the red corpuscles - Counting the white corpuscles. 
Estimation of Haemoglobin . . . . . . . . . 269

Oxygeu capacity of the blood ; the colorimetrie determination of hæmoglobin.

Haemorrilage and Regeneration . . . . . . 273

Physical Aspects of Coagulation . . . . . 273

Physical action of salts in the coagulation of colloidal mixtures - Physical changes in coagulation.

Secretion • • . . . . . . . . . . . . 276

Speed of absorptiou and secretion.

III

\section{RESPIRATION}

Chemistry of Regpiration . . . . . . . . 277

Estimation of oxygen, carbon dioxide, and water.

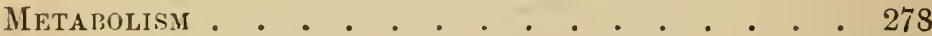

Effect of muscular exercise on the oxygen, carbon rioxide, and water of the respired air - Individual level of proteirl metalsolisn - Nitrogenons equilibrium - Effect of inuscular exercise on proteid metabolism.

\section{PART III}

\section{THE OUTGO OF ENERGY}

\section{I}

Animal Heat

Aximal Heat

Regional temperature - Eflect of lot aud cold drinks on the temperature of the inoutl - Hourly variation $-\mathrm{Re}$ action of cold and warm blooded animals to changes in the external temperature - Chemical aetion the source of animal lieat. 


\section{The Electromotive Phfomomexa of Muscle and Nerve}

The Demarcation Current of Muscle . . . .

Demarcation current of muscle - Stimulation by demarcation current - Interference between demarcation current and stimulating current; polar refusal.

Demarcation Current of Nerve . . . . . . Nerve may be stimulated by its own demarcation current.

Hypotheses regarding the Causation of the Demarcation Currext . . . . . . . . . 297

Action Currest of Muscle . . . . . . . . 302

Rheoscopic frog - Action current in tetanus; stroboscopic method-Action current of human muscle-Action current of heart.

Action Curpent of Nerve . . . . . . . .

Negative variation - Positive variation-Positive after current - Contraction secured with a weaker stimulus than negative variation - Current of action in optic nerve-Errors from unipolar stimulation.

Secretion Current . . . . . . . . . . .

Secretion current from mucous membrane - Negative variation of secretion current.

Electrotonic Currents

Negative variation of electrotonic currents ; positive variation (polarization increment) of polarizing current - Electrotonic current as stimulus.

Electric Fish . . . . . . . . . . . 329 


\section{The Change in Form}

Volume of Contracting Muscle . . . . . . 331

The Single Contraction or Twitch . . . . . 332

Muscle curve - Duration of the several periods - Excitation wave-Contraction wave-Relation of strength of stimulus to form of contraction wave - Influence of load on height of contraction - Influence of temperature on form of contraction - Muscle warmer - Influence of veratrine on form of contraction.

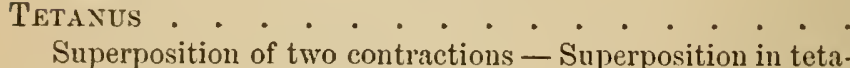
nus - Relation of shortening in a single contraction to shortening in tetanus.

The Isometric JIETHoD

Graduation of isometric spring - Heavy muscle lever Isometric contraction.

\section{Contraction of Human Muscle}

Simple contraction or twitch - Ergograph - Isometric contraction - Artificial tetanus - Natural tetanus.

Smootir Muscle

Spontaneous contractions - Simple contraction - Tetanus.

The Work Done . . . . . . . . . . .

Influence of load on work done-Absolute force of munscle - Total work done; the work adder - Total work done estimated by muscle curve-Time relations of developing energy.

Elasticity and Extensibility . . . . . .

Elasticity and extensibility of a metal spring - Of a rubber band - Of skeletal muscle - Extensibility increased in tetanus.

Fatigue

Skcletal muscle of frog - IIuman skeletal muscle. 
The Central Nervous System

Simple Reflex Actions

The spinal cord a seat of simple reflexes - Influence of afferent impulses on reflex action - Threshold value lower in end organ than in nerve-trunk - Summation of afferent impulses - Segmental arrangement of reflex apparatus - Reflexes in man.

\section{Tendor Reflexes}

Knee jerk - Ankle jerk - Gower's experiment.

Effect of Strychise on Reflex Action. . . . 37 t

Complex Co-ordinated Reflexes . . . . . . . 377

Removal of cerebral hemispheres-Posture, etc. - Balancing experiment - Retinal reflex - Croak reflex.

Apparent Purpose in Reflex Action . . . . 381

Reflex and Reaction Tiye . . . . . . . 382

Reflex time - Reaction time - Reaction time with choice.

Inhibition of Reflexes . . . . . . . . . . 384

Through peripheral afferent nerves - Through central afferent paths; the optic lobes.

The Roots of Spinal Nerves . . . . . . 386

Ludwig's demonstration - Localization of movements at different levels of the spinal cord.

Distribution of Sensory Spinal Nerves . . . 388

Muscular Tonus . . . . . . . . . . . 389

Brondgeest's experiment.

\section{V \\ THE SKIN}

Sensations of Temperature .

Hot and cold spots-Outline-Mechanical stimulation - Chemical stimulation - Electrical stimulation - Temperature after-sensation - Balance between loss and gain 
of heat - Fatigne - Relation of stimulated area to sen

sation - Perception of difference - Relatively insensitive regions.

Sensations of Pressure . . . . . . . . .

Pressure spots-Threshold value-Touch discrimination - Weber's law - After-sensation of pressure Temperature and pressure - Touch illusion; Aristotle's experiment.

\section{VI}

\section{General Sensations}

TICKLE
Irradiation - After image-Topography-Summation Fatigue.

PAIN

Threshold value - Latent period - Summation - Topography - Individual variation - Temperature stimuli.

Moror Sensations . . . . . . . . . . . 400

Julgment of weight-Sensation of effort-Sensation of motion.

\section{VII}

TASTe

TASTE

Threshold value - Topography - Relation of taste to area stimnlated - Electrical stimulation.

\section{VIII}

\section{Intronuction to Pilysiological, Optics}

Reflection from Plane Mirroks . . . . . 403

Angles of incillence and reflection.

Rhilegerion from Concave Mirloms . . . . . 405

I'rincipal focus - Conjugato foci - Virtual image - Construction of iurage from concave nirrors. 
Reflection from Convex Mirrors . . . . 410

Refraction . . . . . . . . . . . . 410

Refraction bx Prisus . . . . . . . . . . 413

Construction of the path of a ray passing through a prism.

Refraction By Convex Lenses . . . . . . . . 416

Principal focus - Estimation of principal focal distance Conjugate foci - Virtual image - Construction of image obtained with convex lens.

Refraction by Concave Lenses . . . . . . . 422

Refraction by Segirents of Cylinders . . . . 422

Refraction through Combined Convex axd CylisDRICAL LENSES. . . . . . . . . . . . . 424

Aberration . . . . . . . . . . . . . . . 426

Spherical aberration by reflection - Spherical aberration

by refraction - Dispersion circles - Myopia - Hypermetropia - Chromatic aberration - Aberration avoided by a diaphragm.

Numbering of Prisms and Lenses. . . . . . 435

Numbering of prisms - Numbering of lenses.

\section{IX}

\section{Refraction in the Eye}

Refraction in the Eye . . . . . . .

The eye as a camera obscura.

The Schematic Ete . . . . . . . . . . 438

Cardinal Points of the Cornea (System A) . . . 440

Construction drawing of System A - Principal focal distances - Construction of image - Calculation of position to conjugate foci.

Cardinal Points of the Crystalline Lexs (System B)

Construction drawing of System B-Optical centre-Nodal points - Principal surfaces - The point $s$ - Principal points - Principal focal distances.

Cardinal Points of the Exe (System C) . . .

Principal surfaces - Nodal points - Principal foci. 
Calculation of the Situation and Size of Dioptric

IMAGES • • • . . • • • • . . • . . 456

Reduced Eye . . . . . . . . . . . . . . 458

Relations of the Visual Axis . . . . . . . 463

Visual angle - Apparent size - Size of retinal imageAcuteness of vision - Smallest perceptible imageMeasurement of visual acuteness.

ACCOMMODATION

Scheiner's experiment - Dispersion circles - Diameter of circles of dispersion-Accommodation line.

Mechanism of Accommodation . . . . . . . .

Narrowing of pupil - Relation of iris to lens - Changes in the lens.

Measurfinent of Accommodation

Far point-Determination of far point-Near point Determination of near point - Range of accommodation.

OPHTHALMOSCOPY

Reflection from retina - Inflnence of angle hetween light and visual axis - Influence of size of pupil - Influence of nearness to pupil - Ophthalmoscope.

Direct Method

Emmetropia - Ametropia; qualitative determinationMeasurement of myopia - Measurement of liypermetropia - Measurement of astigmatism.

Indirect Methon . . . . . . . . 496

\section{$\mathrm{X}$ \\ VISION}

VISION

Mapjping the blind spot - Yellow spot - Field of vision.

Color Bundoness . . . . . . . . . . . . 501

Nethod of exannination and liagnosis. 


\section{$\mathrm{XI}$}

Mechanics of Respiration

Mechanics of Respiration

Artificial scheme - Inspiration - Expiration - Normal respiration - Forced respiration - Obstructed air passages - Asphyxia - Coughing; sneezing - Hiccough Perforation of the pleura.

\section{XII}

\section{The Circulation of the Blood}

The Mechanics of the Circulation . • . . 508 Circulation scheme.

The Conversion of the Internittent into a ConTINUOUS Flow . . . . . . . . . . . . 515

The Relation between Rate of Flow and Width OF BED . . . . . . . . . . . . . . . 519

The Blood-Pressure . . . . . . . . . . . 521

Relation of peripheral resistance to blood-pressure Curve of arterial pressure in the frog - Effect on bloodpressure of increasing the peripheral resistance in the frog-Changes in the stroke of the pump; inhibition of the ventricle - Effect of inhibition of the heart on the blood-pressure in the frog.

The Heart as a Pump. . . . . . . . .

Opening and closing of the valves - Period of outtlow from the ventricle - Sphygmograph tambour - Visible change in form - Graphic record of ventricular contraction.

The Heart Muscle . . . . . . . . . .

All contractions maximal - Staircase contractions - Isolated apex; Bernstein's experiment-Rhythmic contractility of heart muscle - Constant stimulus may cause periodic contraction - Inactive heart muscle still irritable - Refractory period; extra-contraction; compensatory pause - Transmission of the contraction wave in the ventricle; Engelmann's incisions - Transmission 
of the cardiac excitation from auricle to ventricle;

Page Gaskell's block - Tonus - Influence of "loul" on ventricular contraction-Influence of temperature on frequency of contraction - Action of inorganic salts on heart muscle.

The Heart Sounds . . . . . . . . . . . 541

The Pressure-Pulse. . . . . . . . . . . . 543

Frequency - Hardness - Form - Volume - Pressurepulse in the artiticial scheme-Human pressure-pulse curve-Low tension pressure-pulse - Pressure-pulse in anrtic regurgitation - Stenosis of the aortic valve - Incompetence of the mitral valve.

The Volume Pulse . . . . . . . . . 552

\section{XIII}

\section{The Innervation of the Heart and Blood-Vessels}

The Innervation of the Heait and Blood-Vessels 554

The Augaentor Nerves of the Heart . . . . 555

Preparation of the sympathetic - Action of sympathetic on heart.

The Inhubitory Nerves of the Heart . • . 558

Preparation of the vagus nerve - Stimulation of cardiac inhilbitory fibres in vagus trunk - Effect of vagus stimulation on the auriculo-ventricular contraction interval - Irritability of the inhibiterl heart - Intracardiac inhibitory mechanism - Tnhibition by Stamins ligature - Action of nicotine - Atropine - Muscarine - Antagonistic action of muscarine and atropint.

'The Centres of the Heatit Nerves

Inhibitory centre - Augnentor centre - Reflex inhibition of the heart; Goltz's experiment - Reflex angmentation.

The Innervation of the Bloot-Vessels . • . 568

Bulbar centre - Vasomotor functions of the spinal corr Effect of destruction of the spinal corl on the distribution of the blood - Vasomotor fibres leave the corl in the anterior roots of spinal nerves - Vasoconstrictor fbres in the sciatic nerve - Vasorlilator nerves - Reflex vasomotor actions. 


\section{ILLUSTRATIONS}

Diagrams which merely illustrate the grouping of apparatus for a particular experiment are omitted from this list.

Fig.

Page

1. Muscles of left hind limb of frog, dorsal view . . 6

2. Nerve-muscle preparation . . . . . . . . 7

3. Muscle clamp, stand, and nerve-holder . . . . 8

4. Tension indicator . . . . . . . . . . 25

5. Stage electrometer . . . . . . . . . 38

6. Long rheochord . . . . . . . . . . . . 43

7. Square rheochord . . . . . . . . . . 44

8. Simple key . . . . . . . . . . . . 45

9. Short-circniting key . . . . . . . . . . 46

11. Pole-changer, early form . . . . . . . . 49

12. Rocking key . . . . . . . . . . . . 50

14. Inductorium . . . . . . . . . . . 54

15. Platinum electrodes . . . . . . . . . 65

16. Flat-jawed clamp and round-jawed clamp . . . . 66

17. Double clamp . . . . . . . . . . . 66

19. Long paper kymograph . . . . . . . . . . \$2

20. Smoker . . . . . . . . . . . . . 84

21. Light muscle lever . . . . . . . . . . 86

22. Tuning fork . . . . . . . . . . . . 87

23. Non-polarizable electrodes . . . . . . . . . 94

24. Moist chamber . . . . . . . . . . . 95

25. Hind limb of frog, anterior view . . . . . . . 99

25. Gaskell clamp . . . . . . . . . . . . . 103

26. Electro-magnetic signal . . . . . . . . . 105

28. Frog board . . . . . . . . . . . . 112 
Fig.

Page

35. Motor points on the anterior surface of the forearm ancl hand . . . . . . . . . . . 128

36. Motor points on the posterior surface of the forearm 129 and hand

38. Brass electroules

42. Gas chamber, with bottle for generating curion dioxide . . . . . . . . . . 172

43. Sartorius . . . . . . . . . . . . 181

44. Gracilis . . . . . . . . . . . . . 183

4S. Scheme of myomeres in a parallel-fibred muscle . . 298

49. Scheme of myomeres in an oblique section . . . 299

50. Vibrating interupter . . . . . . . . . . 303

51. Vibrating interrupter arranged to make one contact per second . . . . . . . . . $30 t$

53. Heart lever . . . . . . . . . . . 311

54. Scheme of differential rheotome . . . . . . 313

58. Volume tube . . . . . . . . . . . . 332

59. Muscle warmer . . . . . . . . . . . 343

60. Heavy muscle lever . . . . . . . . . . 351

61. Ergograph . . . . . . . . . . . . 354

62. Work adder . . . . . . . . . . . . 359

63. Lantern and optical box . . . . . . . . 404

65. Reduced eye . . . . . . . . . . . . 459

67. Respiration scheme . . . . . . . . . . 504

68. Quantitative circulation scheme . . . . . . . 512

69. Mereury manometer . . . . . . . . . . 523

70. Sphygmograph . . . . . . . . . . 527

71. Sphygmograph tambour . . . . . . . . 528

72. Scheme of sympathetic nerve in frog . . . . . 556

73. Scheme of cervical nerves in frog . . . . . . 558

74. View of brain of frog from above . . . . . 565 


\section{PART I}

THE GENERAL PROPERTIES OF LIVING TISSUES 



\section{PART I \\ THE GENERAL PROPERTIES OF LIVING TISSUES}

I

\section{INTRODUCTION}

UNTIL recent times it was believed that many of the compounds found in the tissues of animals and plants could be made only by the action of organized, $i$. e. living matter. Such compounds were called organic to distinguish them from those found in inorganic or inanimate nature. The forces producing organic compounds were thought to be partly the ordinary chemical and physical processes known to science, and partly certain mystical agencies termed vital forces. The great discovery of Wöhler in 1828 that urea $\left(\mathrm{CO}_{2} \mathrm{NH}_{2}\right)$, a typical organic compound, could be made synthetically in the laboratory, overthrew this conception and was the beginning of a long and fruitful struggle to 
bring the phenomena of living matter within the operation of chemical and physical laws without recourse to the supernatural and occult. According to this new, unified view of nature, which is the foundation of modern physiology, all phenomena, whether animate or inamimate, are alike the expression of chemical and physical processes, some known, some unknown, none of which is fundamentally different from the rest.

The physiologist, therefore, now looks upon the reactions of living matter with the eye of the physicist, and it is of the first importance to beginners in physiology to acquire this point of view. To this end it is desirable to consider living tissues from the standpoint of energy and to divide, even imperfectly, the functions to be studied into those that have to do with the income of energy and those that are active in its outgo. These studies cannot, however, be profitably undertaken without some aequaintance with the general properties of living tissues, such as irritalility and contractility.

We shall begin, therefore, by examining a motor nerve and the muscle in which its fibres are distributed.

The Nerve-Muscle Preparation. - Wrap the frog in the cloth, the head out. Pass one blade 
of the stout scissors between the jaws. Bring this blade to the angle of the jaw, the other blade over the junction of the head and trunk. Cut off the skull with a single closure of the scissors. Thrust the pithing wire into the cranial cavity and then into the vertebral canal, destroying the brain and spinal cord. The frog ceases to move; the muscles are relaxed. Divide the body transversely behind the fore limbs. Remove the viscera. Seize the spinal column with the finger and thumb of one hand, and the skin of the back with the other hand, covered with a cloth to prevent slipping. Draw the hind limbs out of the skin. Lay the limbs down, back uppermost, upon a clean glass plate, which the outside of the frog's skin has not touched. The skin of the frog, like that of the salamander and some other batrachians, is provided with a protective secretion injurious to sensitive tissues. Note on the outside of the thigh the triceps femoris muscle; on the median side, the semi-membranosus; between these, the narrow biceps femoris. (Fig. 1.) Cautiously divide the connective tissue between the semimembranosus and the biceps femoris. On drawing these muscles apart, the sciatic nerve and the femoral vessels will be seen. Clear the nerve with scissors and forceps from the knee 
to the vertebral column. The nerve itself should not be touched with the instruments. Near the pelvis it will be necessary to divide the pyriform and the iliococcygeal muscles: carefully avoid the nerve while do-

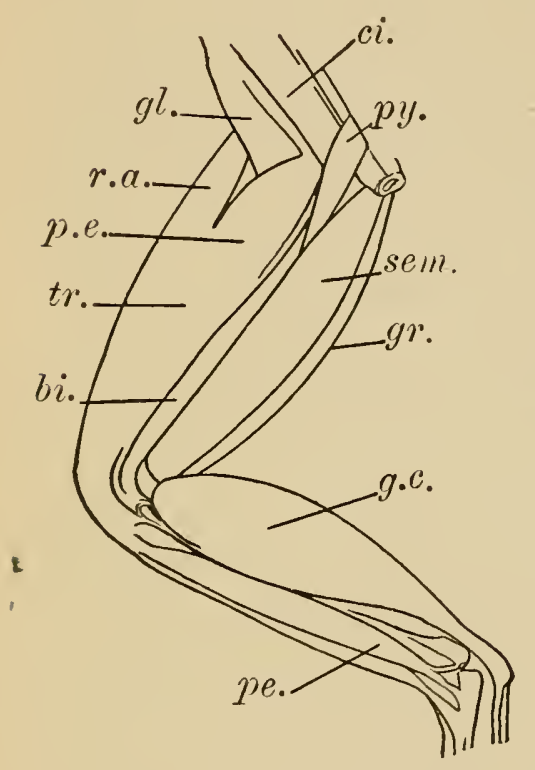
ing this.

With the forceps lift the tip of the urostyle (the 10th vertebra, a long, slender bone which forms the caudal end of the vertebral colu $\mathrm{mn}$ ) and remove the bone with the stout scissors as far as the 9th vertebra. Divide the spinal column transversely between the Fig. 1. Muscles of left him limb of 6 th and 7 th vertebra. frog, dorsal view (Ecker and Wieders- Turn the frog back
heim).

down. Bisect lengthwise the 7th, 8th, and 9th vertebræ. Grasp the half from which the prepared nerve springs and lift it gently, freeing the nerve with the scissors down to the knee.

Pass now to the leg. Cut through the Achilles tendon of the gastrocnemius muscle below 
the thickening at the heel. Free the muscle up to its origin from the femur, taking care not to harm the branch of the nerve which enters the muscle on its posterior surface near the knee. Cut through the tibia about one centimetre from the knee-joint. Clear away the muscles of the thigh from the lower end of the femur, avoiding the sciatic nerve. Cut through the femur about its middle. (Fig. 2.) Lay the sciatic nerve for safety along the gastrocnemius muscle. Fasten the lower fragment of the femur in the jaws of the muscle clamp. Let the whole nerve rest without stretching on the adjustable plate or nerve-holder, the filter paper covering which should be moistened with normal saline

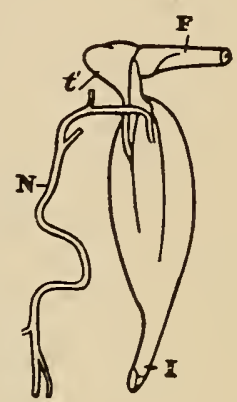

Fig. 2. Nerve-muscle preparation; gastrocnemius muscle and sciatic nerve. $\mathrm{F}$, end of femur; $\mathrm{N}$, sciatic nerve; I, tendo Achillis ; $t^{\mathbf{I}}$, attachment of smaller tendon of gastrocnemius to femur (Handbook for the Physiological

Laboratory). solution ( 0.6 per cent $\mathrm{NaCl}$ ).

Take care that the nerve does not dry between the nerve-holder and the muscle. (Fig. 3.) The filter paper should reach from the nerve-holder to the muscle.

Preliminary Considerations regarding Energy, Stimulation, and Irritability. - Pinch the muscle sharply with the forceps. 
The muscle passes into the active state; it shortens and thickens. The foot, which is rela-

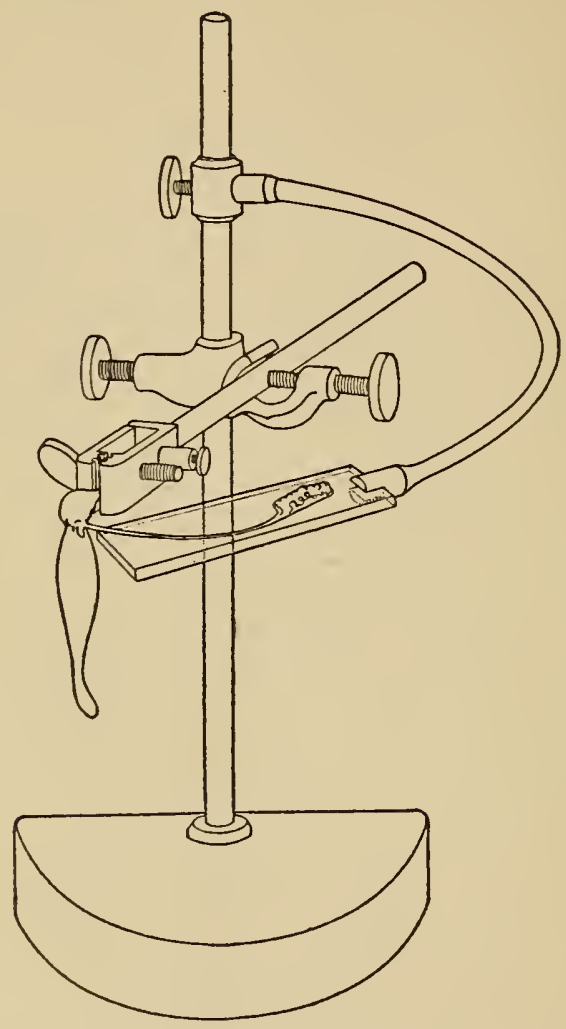

Fig. 3. The muscle clamp, stand, and nerve-holder. The nerve-holder supports the sciatic nerve, together with the portion of the spinal column from which it springs. The handle of the nerve-holder is of thick lead wire which may be bent as desired. The binding post on the muscle clamp provides electrical connection with the upper end of the muscle.

tively less fixed than the leg, is extended. The contraction is followed by a slower relaxation or return to the original form. 
Observe that the mechanical act of pinching caused the resting muscle to become active. Its stored energy was transformed into external, mechanical work, $i$. $e$. the moving of the foot. Not all of the energy set free takes this easily visible form. It will be shown later that much of it is made active as molecular motion, in the form of heat, chemical action, and electricity. Agents which occasion a transformation of energy within the living body are termed stim$u l i$, and tissues which convert energy of one form into energy of another in consequence of stimulation are said to be irritable. All living tissues are alike irritable, but the form in which their kinetic or active energy appears differs with the nature of the tissue. The contrast between muscle and nerve in this respect is especially instructive.

Pinch the end of the nerve.

No change will be seen in the nerve, but the muscle will contract.

Thus, while the most conspicuous form which the energy of muscle takes, when set free, is mechanical, the active nerve does not alter its form, but spends its energy in a molecular change, the nerve impulse, which passes from point to point along the nerve to the muscle, or gland, or other structure connected function- 
ally to the nerve. The effect produced by the nerve impulse depends on the nature of the tissue in which the nerve ends; for example, the energy set free in secreting glands is especially chemical; that set free in the electrical organ of Torpedo is especially electrical. In considering these illustrations of the ways in which the energy of living tissue may be set free, however, two facts should always be kept in mind; first, that by far the greater part of the stored energy of the body is set free as heat; and secondly, that while the several tissues are characterized by the especial prominence of some one form of energy, as contractility in the case of muscle, and the production and conveyance of a nerve impulse in the case of nerve, yet the transformation of energy in each tissue is a complex process, many steps of which, for example heat and chemical action, are common to all living substance.

We have made, then, the fundamental observation that an adequate stimulus will occasion in muscle a conversion of latent energy into mechanical change in form and in the nerve a molecular change that passes along the nerve as a nerve impulse. We must now examine systematically the usual methods of exciting the 
transformation of energy and inquire concerning their effect on muscle and nerve.

\section{Apparates}

Normal saline. Bowl. Cloth. Pithing wire. Scissors. Forceps. Pipette. Glass plate. Cement. Foil. Nerveholder (filter paper). Muscle clamp. Stand. Frog. 


\section{II}

METHODS OF ELECTRICAL STIMULATION

\section{INTRODUCTION}

THe stimulus most usually employed in the laboratory is electricity, because electricity will stimulate when used in quantities which do not destroy the tissues, as do many mechanical, chemical, and thermal stimuli, and because the intensity and duration of the electrical stimulus can be graduated with accuracy. It will be necessary, therefore, to examine with especial care the methods and the results of electrical stimulation. These matters are involved with problems of ionisation, surface tension, osmosis, and other molecular actions highly important in many fields of physiology. Such interdependent phenomena cannot be studied profitably without working hypotheses that shall attempt to relate them as forms of energy.

Kinetic Theory. - The particles of a gas, the simplest state in which matter exists, are identical with its chemical molecules. These particles 
are assumed by Clausius and Maxwell to be in constant rapid motion in all directions. It is this motion which causes the "disappearance" of a gas "set free" in the open air. The molecules of air are also in rapid motion and frequently collide with each other and with those of the escaping gas, but the air molecules are far from filling the space in which they move and the molecules of gas rapidly pass off between them. In their flight, they strike with a measurable force whatever opposes them, be it another flying molecule or some boundary wall. The force of the blow is the "pressure" of the molecule. If the gas be confined by an impermeable wall, that is, a wall the inevitable openings in which are too small for the gas molecules to pass, the sum of the blows of the molecules dashing against this wall will be the total pressure of the gas.

Partial pressure. - If the molecules of a second gas be within the containing vessel, they also will strike the boundary. The force of their blows will be entirely independent of the force of the blows struck by the molecules of the first gas. It is as if black and white balls were thrown at the same time against a wall; their blows would be independent of each other.

The molecules of gas and the confining walls 
are assumed to be perfectly elastic, so that the motion of a molecule is not lost when it collides with another molecule or strikes the wall; the direction of the flight is changed but the swiftness is unimpaired. The speed of the molecules of a gas is inversely proportional to the square root of its density. At $0^{\circ} \mathrm{C}$. the molecule of oxygen moves at the rate of almost eighteen miles per minute.

Diffusion of gases. - The molecules of two gases moving in the same space will intermix or "diffuse," but the rate will be surprisingly slow, for at ordinary pressures the molecules of gases are so near together that they cannot move far without colliding and rebounding. Their progress in any one direction is thus greatly hindered.

Every particle of matter attracts every other particle. Van der Waal assumes that with gases this attraction is proportional to the square of the density of the gas. Where the density is slight, that is, where there are few molecules in proportion to the space in which they move, this attraction need not be taken into account, but where the confining space is small, thus crowding the molecules together, this attraction becomes important. It is still more important in the case of liquids, for in liquids the molecules are much nearer than in a gas. 
Vapor pressure. - Were it not for the attractive or cohesive force just mentioned, the molecules of a liquid would rapidly pass into the space surrounding the liquid and the liquid would soon disappear (evaporation). But the molecules are so close' together that their attraction for each other largely prevents escape. Nevertheless, the motion of many of the molecules at or near the surface of the liquid is sufficient to break through this force of cohesion. These molecules escape and their places are taken by molecules which may in turn escape. Thus evaporation proceeds. In the vapor above the liquid the escaping molecules collide with other molecules and may be driven by the recoil back into the liquid. If the liquid be in a confined space, the number of vapor molecules recoiling into the liquid will increase, partly because they are brought nearer together and collisions are thus more frequent and partly because the molecules rebound from the containing wall. When the number of molecules returning equals the number leaving the liquid, the vapor and the liquid are in equilibrium (saturation). In other words, the vapor tension or pressure or force with which the liquid molecules tend to leave the liquid is balanced by the gas pressure or force with which the gas mole- 
cules strike against the liquid. As the speed of the molecules and therefore their power to escape from the liquid rises with the temperature, the vapcr pressure will also rise with the temperature.

Solution of a gas in a liquid. - A liquid brought near a gas is struck by many of the moving molecules of the gas. Some of these are held by the attractive force of the liquid. Some will at length escape. Finally, as the number of molecules striking the surface of the liquid remains constant at the same pressure, the gas molecules leaving the liquid will equal the number entering (saturation).

Solution of a solid in a liquid. - When a crystalline solid is placed in a liquid solvent, particles escape from the solid and enter the liquid. Some of these particles again enter the solid and are bound by it. Saturation is reached when the number of particles entering and leaving the solid is equal.

Solution tension. - The force with which the particles of the solid pass into the solvent is termed the solution tension. There is equilibrium, $i . e$. saturation, when the solution tension is balanced by the force with which the particles in the solvent return to the solid.

Osmotic Pressure. - It has been found that 
hydrogen gas will pass through the metal palladium when the metal is heated to $200^{\circ} \mathrm{C}$. Nitrogen will not pass through. Falladium at $200^{\circ} \mathrm{C}$. is therefore said to be semi-permeable, i.e. it is permeable to one gas, but not to another. If a tube of palladium at $200^{\circ}$ containing nitrogen gas at a pressure of one half atmosphere be placed in a vessel containing hydrogen gas at a pressure of one atmosphere, the hydrogen will pass through the palladium into the tube until the pressure of the hydrogen inside equals that outside the tube, namely, one atmosphere. The pressure in the tube will now be almost one and one half atmospheres, which is the sum of the partial pressure of the nitrogen (one half atmosphere) and the partial pressure of the hydrogen (one atmosphere), less an error due chiefly to the imperfect permeability of the palladium. Thus the pressure on one side of the palladium will be higher than that on the other side, as may be shown by connecting the tube with a manometer.

Substances in solution may exert a force like the partial pressure of a gas. This force is called osmotic pressure.

Osmotic pressure is measured most readily by the aid of semi-permeable membranes, first used for this purpose by Pfeffer. For demonstration they may be made as follows. Wash a porcelain 


\section{GENeRAL PROPERTIES OF LIVING TISSUES}

diffusion bulb twenty-four hours in running water. Dry the bulb and coat its neck inside and out with paraffin; when this has becume firm, fill the bulb to above the lower edge of the paraffin with solution of copper sulphate $(2.5 \mathrm{gm}$. per litre). Place the bulb in a beaker and pour in a solution of potassium ferrocyanide $(2.1 \mathrm{gm}$. per litre) until the lower edge of the paraffin is covered. Keep the bulb in this solution over night. Where the two solutions meet within the clay wall a precipitation membrane of copper ferrocyanide will form. This membrane is supported by the clay wall. Pour out the contents of the bulb and rinse with cold distilled water. Through such precipitation membranes water and some other solvents will readily pass, while many salts dissolved in the solvent are kept back.

Fill the bulb with one per cent solution of cane sugar. Insert in the neck of the bulb a tightly fitting rubber stopper pierced by a smallbore glass tube about ten feet long. Stand the bulb in distilled water and support the long tube in suitable clamps. The sugar will not pass out through the membrane, but water will pass through it into the bulb, and the solution will rise in the tube at the rate of several inches an hour. The rate is slow because the friction in 
the artificial membrane is great; the osmosis of salts through living membranes may be very rapid. When the liquid in the tube is high enough to balance the force with which the water would pass through the nembrane, the flow ceases. The difference in water level is the osmotic pressure, the analogue of gas pressure. The maximum osmotic pressure is so great that only perfect semi-permeable membranes will support it.

It will be obvious upon reflection that osmotic pressure must be independent of the semi-permeable membrane. The solvent is continuous through the membrane. The only function of the membrane is to render the osmotic pressure visible.

With constant temperature the osmotic pressure is nearly proportional to the concentration of the solution.

$\begin{array}{ccc}\begin{array}{c}\text { Concentration } \\ \text { of solution of } \\ \text { cane-sugar. }\end{array} & \begin{array}{c}\text { Osmotic pressure } \\ \text { in centimetres } \\ \text { of mercury. }\end{array} & \text { Ratio. } \\ \begin{array}{c}\text { Per cent. } \\ 1\end{array} & 53.5 & \\ 2 & 101.6 & 53.5 \\ 2.74 & 151.8 & 50.8 \\ 4 & 208.2 & 55.4 \\ 6 & 307.5 & 52.1 \\ & & 51.3\end{array}$

The osmotic pressure increases as the temperature rises. 
Ten years after these observations were published by Pfeffer, Van't Hoff pointed out the analogy between osmotic pressure and gas pressure. It has just been shown that, if the temperature be constant, the osmotic pressure is proportional to the amount of dissolved substance in a given volume; thus the osmotic pressure of a solution, like that of a gas, varies inversely with the pressure. Moreover, the osmotic pressure, like gas pressure, increases with the temperature. Pfeffer observed that the osmotic pressure of a cane sugar solution at $14.2^{\circ}$ C. was $51 \mathrm{~cm}$. $\mathrm{Hg}$. Assuming that the osmotic pressure, like gas pressure, is proportional to the absolute temperature, Pfeffer then determined by calculation that the osmotic pressure of this same solution at $32.0^{\circ}$ C. should be $54.2 \mathrm{~cm}$. Actual observation gave $54.4 \mathrm{~cm}$.

In short, the osmotic pressure of a dissolved substance is numerically equal to the pressure which the substance would exert were it present as a gas.

Plasmolysis. Isotony. - The cells of Tradescantia discolor possess a strong outer envelope permeable by both water and salts, and a thin inner envelope permeable by water, but not by salts. The cell is filled with an aqueous solution of glucose, salts of malic acid, etc. The osmotic 
pressure of the contents is from four to six atmospheres. When the cell is placed in water, the water penetrates both envelopes and the cell swells so far as the resistant outer envelope may permit. In a concentrated salt solution, the osmotic pressure pushes the inner envelope away from the outer envelope, contracting the volume of the cell contents (plasmolysis), and water leaves the cell until the concentration of the cell contents equals that of the surrounding solution. Solutions whose concentration is such that cells immersed in them are not deformed must possess the same osmotic pressure. Such solutions are termed isotonic or isosmotic.

Estimation of Osmotic Pressure in the Blood Serum. 1. The method of de Vries. ${ }^{1}$ - Make a section along the midrib of the violet side of the leaf of Tradescantia discolor and two other sections one on each side, parallel to and about $2 \mathrm{~mm}$. from the first. Now make transverse sections across the three vertical sections about $5 \mathrm{~mm}$. apart, and a final very thin section parallel to the surface. Place some of these thin tangential sections in the serum diluted with 20 per cent of water, and others in salt solutions of different concentrations $(0.60,0.65,0.70,0.75,0.80$

1 DE VRIEs: Zeitschrift für physikalische Chemie, 1888, ii, p. 419. 
per cent). From time to time remove the sections and observe under the microscope whether plasmolysis is present. The serous fluid that causes plasmolysis in half the cells immersed in it, is isotonic or "normal" with the salt solution having the same effect.

For example, if there were used 5 c.c. serum +1 c.c. water with 0.8 per cent solution of sodium chloride, the original serum would be isotonic with a sodium chloride solution of $\frac{5+1}{5} \times 0.8$ $=0.96$ per cent.

Should the osmotic pressure of the serum be too slight to call forth plasmolysis in the cells employed, add to the serum measured quantities of a strong saline solution, e.g. 5 per cent sodium chloride solution. ${ }^{1}$

2. The blood-corpuscle method of Hamburger. ${ }^{2}$ - Place 5 c.c. serum in each of six test-tubes. To these add from a burette $3.1,3.0,2.9,2.8,2.7$, and 2.6 c.c. water, respectively. Into each tube let fall three drops of defibrinated blood, and mix

1 Hamburger : Osmotiseher Druck und Ionenlehre, 1902, i, 1. 438.

The cells of Tradescantia cannot be used for the measurement of osmotic pressure in acil solntions. For the urine, the cells of liegonia manicata should be userl.

2 Hamburiere: Loc. cit. pp. 185 and 439. Also Archiv fïr 1'hysiologie, 1887, p. 31. 
by shaking, taking care that the mixture does not foam too much.

In each of six other test-tubes place 8 c.c. sodium chloride solution of $0.62,0.61,0.60,0.59$, 0.58 , and 0.57 per cent, respectively. Into each tube let fall three drops of the same defibrinated blood. Mix as before. After some hours the red corpuscles will have settled to the bottom in all the tubes.

In the first series the clear fluid will be red in some of the tubes. For example, the fluid in the tubes diluted with $3.1,3.0$, and 2.9 c.c. water may be red, while in the three other tubes it may not be red. In this case the mixture of 5 c.c. serum +2.9 c.c. water has caused the hæmoglobin to escape from the corpuscles ("laked" the blood), while the mixture of 5 c.c. serum +2.8 c.c. water has not accomplished this.

If the tubes with salt solution be now examined, escaped hæmoglobin will be found only in the 0.58 per cent and the weaker solutions. Consequently the mixture of 5 c.c. serum $+\frac{2.9+2.8}{2}$ water is isotonic with a sodium chloride solution of $\frac{0.59+0.85}{2}=0.585$ per cent. Hence the undiluted serum is isotonic with a sodium chloride solution of $\frac{5+2.85}{5} \times 0.585=0.92$ per cent. 
In a solution of this concentration the red bloodcorpuscles of mammals are in osmotic equilibrium. ${ }^{1}$ The isotonic (isosmotic or normal)

1 In this calculation it is assumed that the osmotic pressure is proportional to the concentration. This is not strictly correct. When serum is diluted with an equal volume of water, the osmotic pressure of the resulting liquil is not merely half so large as before, but is somewhat greater than one half the original pressure. For when the water is added, a part of the molecules not yet dissociated are split into ions and each ion has an osmotic pressure equal to that of an undissociated molecnle. If the serum upon dilution with water followed the same dissociation curve as the sodium chloride solution of 0.92 per cent, the above method of calculation would be strictly correct. This is, however, not the case ; on the contrary, the dissociation curves separate from each other. But this deviation, in the case of the dilutions considered here, lies within the limit of errors of observation, probahly because the osnotic pressure of the serum is due chiefly to sodium chloride (Hamburger, p. 186).

The above calculation also leaves out of account the fact that the blood-corpuscles are permeable for anions. It is evident that the exchange of ions between blood-corpuscles and surrounding liquid will differ according as this liquid is dilute serum or pure sodium chloride solution. For the partial tension of the ions in the two solutions is not the same. The partial pressure of the $\mathrm{Cl}^{\prime}$ ions in the 0.6 per cent sodium chloride solution is greater than in the serum diluted with 60 per cent water; while in the serum there is a pressure of $\mathrm{CO}^{\prime \prime \prime}$ ions, lacking in the sodium chloride solution. Moreover, the blood-corpuscles placed in these liquids acquire a different composition. The osmotic pressure of the blood-corpuscles is increased by immersion in sodium chloride solution.

Dissociation and permeability act with opposite sign, balancing each other. That the blood-corpuscle method is practically 
concentration for the corpuscles of frog's blood is 0.6 per cent.

Surface Tension. - The osmotic pressure of solutions is very great. In the ordinary reagents of the laboratory it amounts to many atmospheres. Obviously, such solutions could not be kept in thin glass beakers or bottles were these enormous pressures not restrained by some opposing force. This opposing force is surface tension, which acts with a pressure of probably hundreds of atmospheres upon the free surface of a liquid, whether that surface be bounded by air or glass. The semi-permeable membrane is filled with liquid, and therefore does not present a free

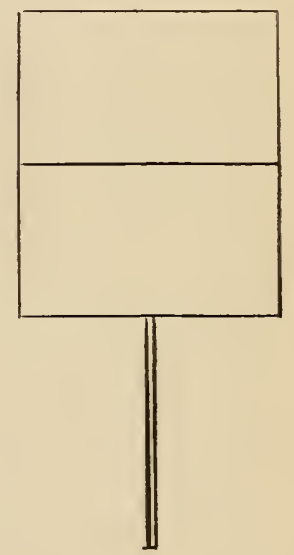

Fig. 4. The tension indicator. surface.

1. A thick wire is bent to enclose a rightangled space and the end prolonged for a handle. (Fig. 1.) A very fine slack-wire divides the space in halves.

accurate may be shown by comparison with the method of estimating osmotic pressure by the depression in the freezingpoint of the solution, which is proportional to the concentration of the solution (p. 439). 
Dip the tension indicator into a soap solution.

A thin layer of the solution will span the frame on both sides of the dividing wire. The tension of the membrane on one side of the dividing wire compensates that on the other and the wire will not move.

Hold the frame in a vertical position. The cross wire will sink slightly, owing to the force of gravity. Absorb the lower membrane with filter paper.

The cross wire will at once be lifted against the force of gravity by the surface tension of the remaining membrane. Destroy the remaining membrane and the wire will fall again.

2. Strew lycopodium ${ }^{1}$ upon a water surface. In the centre place a few drops of alcohol.

The lycopodium will be driven in all directions. The surface tension of alcohol is less than that of water. The water-air surface and the alcohol-air surface are therefore not in equilibrium. The water-air surface contracts violently, and the alcohol-air surface expands, producing the strong currents made visible by the motion of the lycopodium.

Electrolysis. - 1. Connect two dry cells in series (carbon of one cell connected with zinc of other). Attach wires to the terminal zinc and

1 Be careful not to bring the lycopodium near a flame. 
carbon. Touch the ends of the wires together. An electric current passes, as evidenced by the spark when the contact is made or broken, but no material change can be observed in the metallic conductor.

2. Place two pieces of platinum foil in a solution of copper sulphate and connect them to the terminal zine and carbon as before. Copper will be deposited on the platinum connected with the zinc, and oxygen will be deposited on the platinum connected with the carbon. Thus the passage of the current through the conducting solution or electrolyte has caused a change in the solution, evidenced by a movement of ponderable matter.

Faraday discovered that the quantity of matter moved by the.current was always proportional to the quantity of the current, independent of its strength or speed. In the electrolysis of hydrochloric acid 96,500 coulombs ${ }^{1}$ will set free $1 \mathrm{gm}$. of hydrogen. Faraday also discovered that the same quantity of current $(96,500$ coulombs) which carries $1 \mathrm{gm}$. of hydrogen will carry the chemical equivalent of any other sub-

1 A coulomb (ampere) of electricity is the quantity of current which, when passed through a solution of silver nitrate in water, deposits silver on the cathode, or negative pole, at the rate of 0.001118 gram per second. 
stance, for example $35.5 \mathrm{gm}$. of chlorine. In the above experiment on the electrolysis of copper sulphate solution, each 31.5 grams of copper moved to the zinc pole requires the passage of exactly 96,500 coulombs. The particles of matter travelling in the solution toward the anode were called by Faraday the anions and those travelling toward the cathode were called cations. On reaching the poles the ions give up their electric charge and resume their simple chemical nature. Thus each 31.5 grams of copper ions, on reaching the cathode, gives up 96,500 coulombs of electricity and becomes ordinary copper again, and each 96 grams of sulphate ions gives up 96,500 coulombs and becomes the ordinary radical $\mathrm{SO}_{4}$. This radical cannot exist uncombined in water, but forms sulphuric acid. $\mathrm{SO}_{4}+\mathrm{H}_{2} \mathrm{O}=\mathrm{H}_{2} \mathrm{SO}_{4}$ $+\mathrm{O}$. Thus the liquid around the anode becomes acid, and actual measurement has shown that the quantity of sulphuric acid formed at the anode is exactly equivalent to the quantity of copper deposited on the cathode.

The ions move very slowly. The high viscosity or internal friction of the liquid opposes a great resistance to the movement of ions as well as to the osmosis of dissolved substances. To drive $1 \mathrm{gm}$. of hydrion (OH) through pure water at the rate of $1 \mathrm{~cm}$. per second, a force equal to 
320,000 tons' weight is required. To diffuse $1 \mathrm{gm}$. urea through pure water at the rate of $1 \mathrm{~cm}$. per second, 40,000 tons' weight is required. In dilute aqueous solutions at $18^{\circ} \mathrm{C}$. a difference of potential of 1 volt between electrodes $1 \mathrm{~cm}$. apart will drive the cation $\mathrm{H} 10.8 \mathrm{~cm}$. per hour, the cation $\mathrm{Na} 1.26 \mathrm{~cm}$, the anion $\mathrm{OH} 5.6 \mathrm{~cm}$, and the anion $\mathrm{Cl} 2.12 \mathrm{~cm}$. per hour.

As the ions move so slowly and as the products of electrolysis appear at each electrode the moment the current is made, it is evident that the ions which inmediately appear at the anode cannot be derived from the same molecules as the ions which simultaneously appear at the cathode.

"Clausius explains this in the following way: According to the theory of molecular motion, of which he has himself been the chief founder, every molecule of the fluid is moving in an exceedingly irregular manner, being driven first one way and then another by the impacts of other molecules which are also in a state of agitation.

"This molecular agitation goes on at all times independently of the action of electromotive force. The diffusion of one fluid through another is brought about by this molecular agitation, which increases in velocity as the temperature rises. The agitation being exceedingly irregular, the encounters of the molecules take place with various 
degrees of violence, and it is probable that even at low temperatures some of the encounters are so violent that one or both of the compound molecules are split up into their constituents. Each of these constituent molecules now knocks about among the rest till it meets with another molecule of the opposite kind, and unites with it to form a new molecule of the compound. In every compound, therefore, a certain proportion of the molecules at any instant are broken up into their constituent atoms.

"Now Clausius supposes that it is on the constituent molecules in their intervals of freedom that the electromotive force acts, deflecting them slightly from the paths they would otherwise have followed, and causing the positive constituents to travel, on the whole, more in the positive than in the negative direction, and the negative constituents more in the negative direction than in the positive. The electromotive force, therefore, does not produce the disruptions and reunions of the molecules, but, finding these disruptions and reunions already going on, it influences the motion of the constituents during their intervals of freedom." I

1 Quoted from Clerk Maxwell by Walker in his admirable. "Introduction to Physical Chemistry," a book to which the present writer is much indebted. 
Views of Arrhenius. - It was Arrhenius who made these ideas regarding the dissociation of the molecules of solutions into ions quantitative and thus precise. Feeble electrolytes, i. e. poor conductors of electricity, are but slightly dissociated, whereas solutions that readily conduct electricity are largely dissociated. Ionisation may be measured by the degree of conductivity. Undissociated molecules carry no electricity. Each univalent ion has the same load : 96,500 coulombs.

Conductivity depends on the number of the ions and upon their speed. In this connection the following observation is of interest. If successive quantities of pure water be added to a salt solution, the conductivity will increase as the dilution increases. The first factor to be considered here is the viscosity of the solution, for the viscosity determines the resistance of the solution to the passage of ions, and thus determines the speed of the ions. As the addition of water continues a point is soon reached at which the amount of water in the solution is so great in relation to the amount of salt that the water may be regarded practically as pure. The addition of more water should not then appreciably affect the viscosity and thus the speed of the ions. If the conductivity were related solely to the speed of the ions, the conductivity should not then 
increase upon this further addition of water. Observation shows that it does increase. It must therefore depend on the degree of dissociation, $i$. $e$. the number of ions.

When the solution is heated, its viscosity and thus its fluid friction diminishes; the ions pass through it with less difficulty and the conductivity rises. Non-conducting substances added to aqueous solutions may affect both the number and the speed of the ions. The first additions of alcohol to a dilute solution increase the viscidity, and lessen the speed of the ions; after a certain limit, further additions have little effect on the speed, but markedly lessen the number of the ions, i.e. the degree of ionisation or dissociation. Comparisons of electrical conductivity in solutions of different concentration can be made only when the rate at which the ions move remains the same; in other words, the salt, the solvent, and the temperature must be the same in the two solutions.

Electrolytic solution Pressure. - When a salt is placed in water it dissolves until its solution pressure or tendency to pass into solution is balanced by the osmotic pressure of the dissolved particles. If the osmotic pressure exceed the solution pressure, salt will be deposited. As the salt dissolves, positive and negative ions are 
formed in equivalent quantities, so that there is no difference in electrical pressure.

When metallic zinc is placed in dilute sulphuric acid, it dissolves, and positively charged zine ions enter the solvent, but no negative ions are formed at the same time. The solution next the metal becomes statically charged with positive electricity, and in consequence the zinc itself becomes negatively charged. The electrical stress thereby produced compensates the difference between the osmotic pressure of the zinc ion and the electrolytic solution pressure of the zinc.

When copper is placed in a solution of copper sulphate, the osmotic pressure of the metal ion exceeds the electrolytic solution pressure of the metal and metallic copper is deposited on the surface of the electrode. The metal becomes positively charged and the solution negatively charged through the sulphanions that gather at the layer of solution next the metal. This process continues until the electrical stress balances the difference between the actual osmotic pressure and the electrolytic solution pressure.

The electrical double layer about each electrode is of only molecular thickness, so that the solution or deposition of an extremely small quantity of metal is sufficient to establish equilibrium. 
In galvanic cells, in which two metals are immersed in one or more electrolytes, there are three sources of electromotive force: the junction of the metals with the electrolytes, the junction of the two metallic conductors, and the junction of the electrolytes. The junction of the metals with the electrolytes is the principal source; the electromotive force produced by the junction of the metals is slight, and the ions of different neutral salts move at not far from the same speed, so that the electromotive force at the junction of the electrolytes is also relatively small.

The Electrometer, the Rheocord, and THE CelL

In order to study differences in electrical potential, 1 a galvanometer or some other elec-

1 'The difference of potential may be compared to the difference of water level between a reservoir and its distributing pipes. It produces an electromotive foree, comparable to the force which moves the water from the higher to the lower level. The unit of electrical pressure is the volt. The flow through an hydraulic system is measured by the quantity of water passing any point in a given time; similarly the quantity of eleetricity is the amount that flows through a cross-section of the conductor in a given time. The unit of yuantity is the ampere. Electricity passing through a conductor meets with a resistance which becomes greater as the cross-section of the conductor. 
trometer is necessary. In the galvanometer, the points of different potential are connected by a coil of wire near which is suspended a magnet. When the circuit is completed, the electrical energy acts on the suspended magnet by induction, and deflects it to an extent proportionate to the difference of potential. In the capillary electrometer, which is the electrometer preferred here, a capillary tube filled with mercury and sulphuric acid dips in a wider tube which contains sulphuric acid. The points the potential of which is to be measured are connected with the mercury and the acid respectively. When the connection is made, the tension of the surface of mercury in contact with the acid changes, causing the mercury to move in the capillary. The change in surface tension is proportional to

diminishes, just as water can be forced more easily through wide channels than through narrow ones. The unit of electrical resistance is the ohm. The precise definition of these units is as follows :

A volt is the electromotive force that, steadily applied to a conductor whose resistance is one international ohm, will produce a current of one international ampere. The practical ampere (coulomb) is the unvarying current which, when passed through a solution of nitrate of silver in water, deposits silver on the cathode, or negative pole, at the rate of 0.001118 gram per second. The $\mathrm{ohm}$ is the resistance offered to an unvarying electrical current by a column of mercury at the temperature of melting ice, 14.4521 grams in mass, of a constant crosssectional area, and of the length of 106.3 centimetres. 
the difference in potential. The action of the instrument will be more clear from the following experiments.

\section{Surface Tension altered by Electrical Energy. -} In a small porcelain evaporating dish place a globule of mercury about one inch in diameter.

The colhesion of the mercury is stronger than the attraction between the mercury and porcelain, - the mercury does not "wet" the porcelain. The free surface of the mercury is curved and not plane, as it would be were the molecules acted upon by the force of gravity alone. Obviously the spreading of the mercury is resisted by some force that strives to make the drop spherical, $i$. e. to make the surface as small as possible.

This force is called the surface tension. It is the attraction which the molecules beneath the surface exert on the side of the surface layer next them. The form of the drop is the result of the equilibrium between these opposing forces (Thomas Young, 1804).

Cover the mercury one centimetre deep with 5 per cent sulphuric acid. Note carefully the degree of convexity. Add a trace of potassium bichromate. The drop will flatten slightly.

When a metal is placed in an electrolyte, a difference of potential is created at the surfaces in contact. If the metal is positive compared 
with the electrolyte, an immeasurably thin layer of positively electrified molecules may be said to coat its surface, and in the electrolyte a parallel layer of negatively electrified molecules will collect. On every side of the parallel layer electricity of the same sign will be repelled. In the case of a liquid metal, for example mercury, the form of the surface will be altered, for the repulsion of like electricities will tend to stretch the surface layer, and will thus oppose the surface tension. The new form which the surface will take is the equilibrium between the electrical energy and the surface tension (Helmholtz). If this equilibrim is changed by the introduction of new electrical energy, the curvature of the surface will change (Henry).

Fasten an iron wire in the muscle clamp and clamp the latter to the stand. Bring the wire over the mercury and lower the muscle clamp until the wire just touches the edge of the mercury. Fix the clamp in this position.

The instant the two metals touch (iron and mercury in chromic acid solution) the existing difference of potential will be altered. The surface tension will thereby be increased and the globule will become more convex. This movement withdraws the margin of the globule from the iron and the globule flattens again, which 
brings it again into contact with the iron. This play is repeated until the chromic acid is all reduced to chromic sulphate.

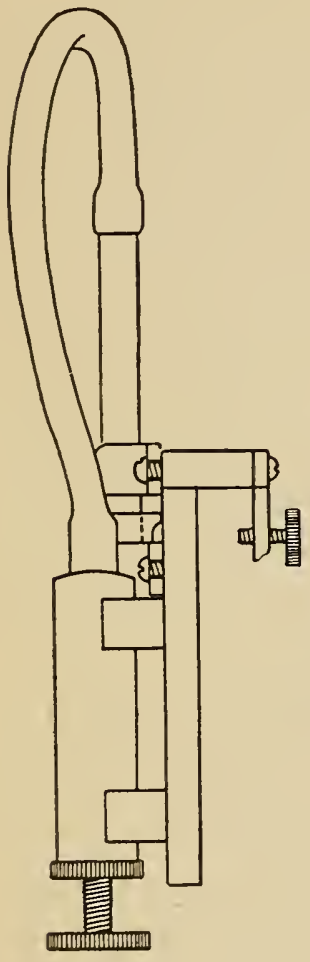

A

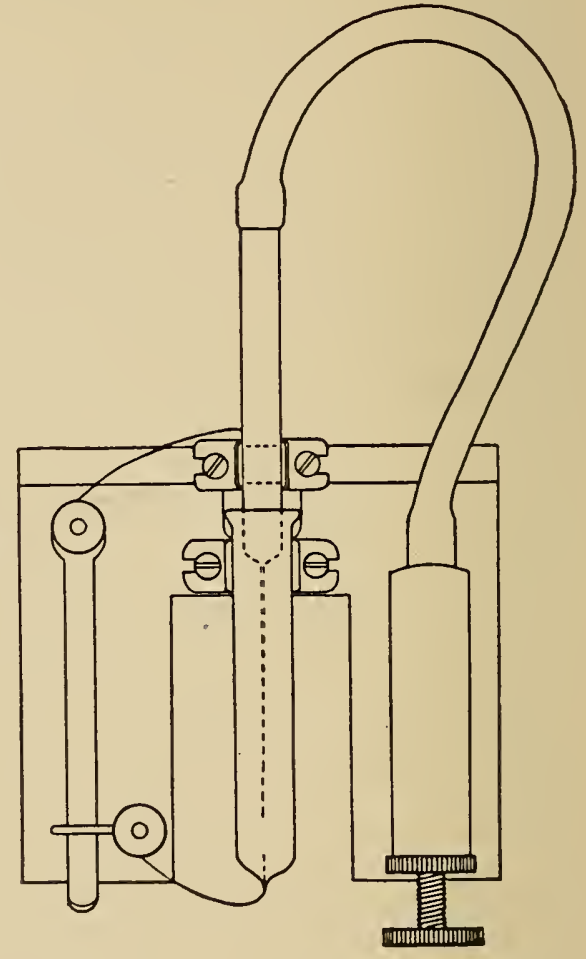

B

Fig. 5. Stage electrometer; ${ }^{1}$ about three-sevenths the actual size. A, Side view. B, Front view.

The Electrometer. - The electrometer consists of a vertical tube drawn out at the lower end into a fine capillary and filled with mercury. (Fig. 5.) The

1 Science, 1905 , xxii, p. 602. 
upper end of the tube is joined to a cylinder in which a piston is moved by a screw, thus making pressure on the mercury column. The end of the capillary dips in a reservoir containing twenty per cent sulphuric acid. Platinum wires lead from the acid reservoir and the mercury in the capillary to convenient binding posts. The platinum wire should never touch the acid, but should be protected by a covering of mercury. When mercury is placed in the vertical tube it enters the capillary until the weight of the column of mercury is balanced by the surface tension, which is inversely proportional to the diameter of the tube. If the capillary be now dipped in the reservoir containing the sulphuric acid, and the piston driven upward by its screw, mercury will be forced out of the capillary into the acid; and on lowering the pressure the mercury will retreat within the capillary, drawing the acid after it. Numerous adrantages are presented by this form of electrometer. It fits the stage of the microscope. The microscope need not be tilted very far, and the observer is therefore in. a comfortable position. The position of the electrometer on the stage may readily be changer. All the parts near the acid are of hard rubber, thus excluding currents that might arise from acid touching metal parts. The acid tube is flanged so that the acid cannot creep out along the capillary tube. The capillary can easily be brought against the wall of the acid tube. The tube from which the capillary springs descends within the acid tube, thus protecting the capillary 
against breakage. Either tube may at once be removed from its holder. The platinum wires extend to the binding post, and are not simply short pieces soldered to copper wire. The wire to the capillary tube extends to the bottom of the tube, thus maintaining the contact until all the mercury in the tube is used.

About one cubic centimetre of paraffin oil should be placed above the piston. Only absolutely clean double-distilled mercury should be used.

As the mercury in the capillary is kept from falling by the surface tension, it is obvious that whatever increases or diminishes the surface tension, for example an electric current, will raise or lower in corresponding measure the mercury in the capillary. The alteration in surface tension is accompanied by the movement of ions between the meniscus and the remaining electrode of the electrometer (the mercury in the acid reservoir). In practice it is found that this movement can be neither very rapid nor long continued, without injuring the sensitiveness of the instrument. The potential difference from even a single element (Daniell or dry cell) is far too large to be used safely. It is advisable to employ a potential divider, or rheochord, which shall permit only a fraction of the original potential (not more than 0.1 volt) to reach the 
electrometer. The platinum should never come in contact with the acid.

The electrometer should be kept short-circuited, except during an observation, so that the capillary and the mercury in the reservoir may always be connected through a conductor. The short-circuit key' is shown in Fig. 5, B. A strip of spring brass connected with one of the binding posts of the electrometer rests against a second piece of brass connected with the other binding post, except when depressed by the finger. The point of higher potential, when known, should always be connected with the capillary.

When the capillary electrometer is connected with two points of unlike potential the meniscus is displaced. The pressure necessary to bring it back to its original position is proportional to the electromotive force that displaced the meniscus. Thus by comnecting the electrometer with known differences of potential it may be experimentally graduated. In practice, the relation between the pressure and the potential must frequently be redetermined. It is usually easier to measure differences of potential, such as the demarcation current of nerve or muscle, by compensation (Fig. 47, p. 294). In this method the electromotive force of the demarcation current 
is measured in fractions of a Daniell cell, or any other constant element, by bringing into the same circuit with the current of injury, but in an opposite direction, so much of the current from the cell as will exactly balance the current of injury, $i$. e. so much as will keep the meniscus of the electrometer from moving in either a positive or negative direction when connected with the circuit.

Advantages of the Electrometcr. - The mass of mercury displaced in the movement of the meniscus is very small, and the distance through which it is moved is short. Hence the inertia of pasition is easily overcome and the inertia of motion (which is proportionate to the mass times the square of the velocity) is practically wanting. The absence of inertia errors, the almost instantaneous quickness with which the meniscus takes its new position, the ease with which slight electromotive forces $\left(\frac{1}{10000} \mathrm{volt}\right)$ may be measured, and simplicity of construction, are the principal advantages of this admirable instrument.

The Rheochord. - If two poles of a cell or other points of different potential be joined by a well-drawn wire, the potential through the wire will fall uniformly from the anode to the cathode. The greater the resistance in the wire, the more uniform will be the fall in potential. 
The Long Rheochord. - In the long rheochord (Fig. 6) a metre rule is screwed upon a wood base. At each end is a binding post. To post 0 is fastened the end of an unbroken German-silver wire twenty metres in length. This wire is carried along the metre stick to the second post, 1, then wound upon a spool, and the end fastened to a third binding

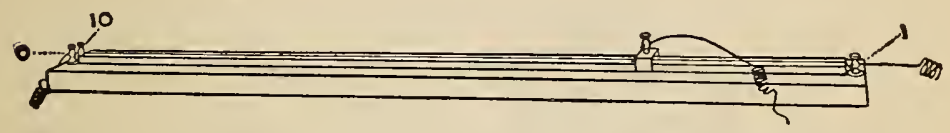

Fig. 6. The long rheochord; about one-thirteenth the original size.

post. The wire upon the metre rule is bare, the remaining nineteen metres silk-covered. A convenient spring contact with binding post slides along the metre rule.

The Square Rheochord. - Upon a block of hard maple, $12.5 \mathrm{~cm}$. square, is placed a centimetre scale beginning at the 0-post shown on the left side of Fig. 7 and ending at the 1 -metre post visible in the background to the left. For the sake of clearness the numbers on this scale have been omitted from the figure. Along the scale, between these two posts, is stretched the first metre of a continuous German-silver wire, $0.26 \mathrm{~mm}$. in diameter and twenty metres long. The remaining nineteen metres of this wire are coiled upon a spool, and the free end is fastened to the twenty-metre post shown in the background to the right of Fig. 7 . One of the posts may be turned, in 
order to keep the wire taut, in case changes of temperature have caused it to lengthen. (This device is not shown in Fig. 7.) The under surface of the contact block is bevelled so that the metal touches the wire only with one edge; the opposite edge is supported by a piece of hard rubber.

A flexible cable leads from the contact block to the binding post shown in the foreground to the right.

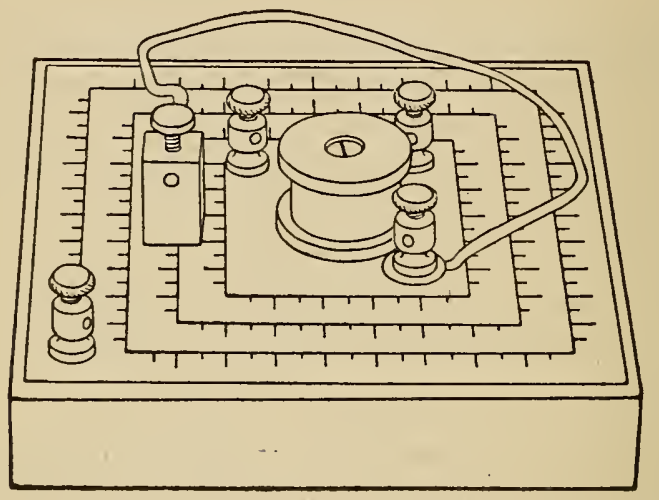

Fig. 7. The square rheochord ; two-fifths the actual size. ${ }^{1}$

The resistance in the 20 metres of thin German-silver wire is so great (about $184 \mathrm{ohms}$ ) that the internal resistance of the element furnishing the electromotive force, together with the resistance of the large copper connecting-wires, practically disappears for such measurements as we shall need to make. As the fall of potential is uniform throughout the 20 metres, the differ-

1 American Journal of Physiology, 1903, viii, p. xli. 
ence of potential between post 0 and post 1 will be practically one-twentieth the electromotive force of the element. Thus when the sliding contact is at post 1, the capillary electrometer receives one-twentieth the electromotive force of the element. By moving the slider from post 1 towards post 0 , any desired fraction of this onetwentieth may be measured by the electrometer.

The Simple Key. - A copper bar with hard rubber handle is pivoted at one end in a brass post with binding screw for electrical connection (Fig. 8). Near the other end of the bar is a platinum pin, which, when the key is closed, rests upon a platinum plate borne upon a second binding post.

The contact bar is held against the contact plate

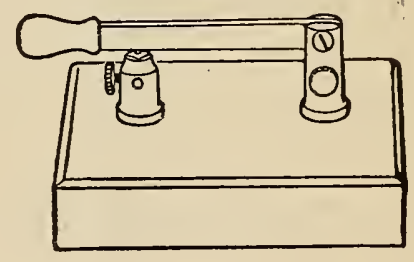

Fig. S. The simple key; about three-eighths the actual size. The wire spring which presses the bar against the contact plate is not shown.

partly by its own weight and partly by a wire spring not shown in Fig. 8. When it is desired to break the circuit the contact bar is turned back.

Many experiments in physiology require stimuli of uniform intensity. Variations in the make or break of the current due to faults in the contacts of the key in the primary circuit are a frequent source of error. With the key described here the break in the circuit may be made practically uniform. 
The Short-circuiting Key. - Two strips of brass, provided with a binding post at each end, are fastened to a block of dark slate (Fig. 9). At the centre of one strip is a post in which is pivoted a copper bar ending in a hard-rubber handle. The bar may be lowered between edges of spring brass.

Polarization. - Connect a platinum and a zinc ${ }^{1}$ plate through a simple key with posts 0 and 20

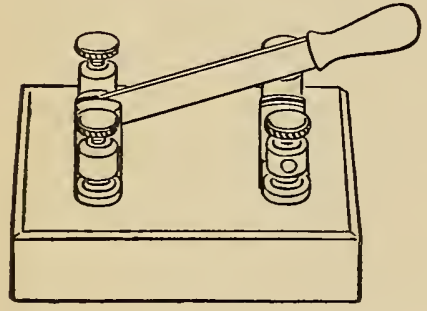

Fig. 9. The short-circuiting key; about three-eighths the actual size.

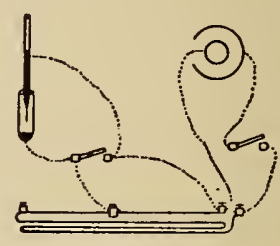

Fig. 10.

of the rheochord as shown in Fig. 10. Connect the zero post and the slider with the capillary electrometer through a short-circuiting key.

1 It will be observed that the zinc is amalgamated. Chemically pure zinc does not need amalgamation. Commercial zinc contains iron, arsenic, etc., as impurities. The contact of unamalgamated zinc and these dissimilar metals with an electrolyte creates a difference of potential, and parasitic currents run from the zinc to the foreign metals. These currents are prevented by covering the impurities with zinc amalgam, the electromotive properties of which, toward sulphuric acid, are those of pure zinc. As the zinc in the amalgam dissolves out, the film of mercury unites with fresh zinc. Zine is amalgamated best by 
Bring the capillary into the field of the microscope (Leitz objective 3, micrometer ocular), parallel to the micrometer scale. The end of the tube should be just visible at the upper margin of the field. If the meniscus is not visible, turn the pressure screw slowly to the right until the meniscus enters the field. Note the position of the meniscus on the scale. Close the battery key. Let an assistant place the metals in a beaker containing solution of sodium chloride. Open the short-circuiting key of the electrometer.

When the metals touch the electrolyte a difference in potential will be set up, and the meniscus will move in the capillary.

Note the number of divisions of the scale traversed by the meniscus. Close the electrometer key. Wait several minutes.

Now bring the meniscus back to its original position on the scale. Open the electrometer key.

The meniscus will move to a much slighter extent than when the circuit was first made.

adding 4 per cent of mercury to the molten zinc before casting; or the zinc may be dipped in 10 per cent sulphuric acid to clean it, and mercury rubbed over the surface with a brush or a stick padded with eloth; or the zinc may be dipped in a solution from which the mercury will deposit on the zinc. Formula for amalgamating fluid: warm gently 4 parts mercury in 5 parts concentrated nitric acid and 15 parts concentrated hydrochloric acid until dissolved, and then add 20 parts more of concentrated. hydrochloric acid. 
As the displacement of the meniscus is proportional to the electromotive force of the cell, it is obvious that the latter has rapidly diminished. The solution contains the ions of water as well as those of the salt. When the circuit between the platinum and zinc is completed the cations $\mathrm{H}^{+}$and $\mathrm{Na}^{+}$move towards the cathode. There the more easily de-ionized $\mathrm{H}^{+}$yields up its electricity, and hydrogen appears on the cathode. The corresponding quantity of electricity is conveyed into the solution at the anode by ionization of the zinc. The deposition of hydrogen on the negative plate checks the electromotive force setting from the zinc to the platinum in two ways: first, because gas is a bad conductor, and the effective surface of the platinum is thereby diminished by the bubbles collecting on it; and secondly, because hydrogen is electropositive, and creates an electromotive force in the direction from platinum to zinc, and thus "polarizes" the cell. This new electromotive force opposes the original current from zinc to platinum.

The Daniell Cell. - Daniell discovered an electro-chemical method of avoiding polarization, and thus was able to construct a cell that would furnish a current of unvarying strength. In the Daniell cell the two metals employed are zinc 
and copper. The amalgamated zinc is placed in a porous cup filled with dilute sulphuric acid. The copper is placed in a solution of copper sulphate kept saturated by crystals of the salt. When the circuit is closed, the zinc "dissolves" in the sulphuric acid, carrying with it the electricity with wich the zinc ions are charged. The electricity is carried through the solution by the migration first of hydrogen and then of

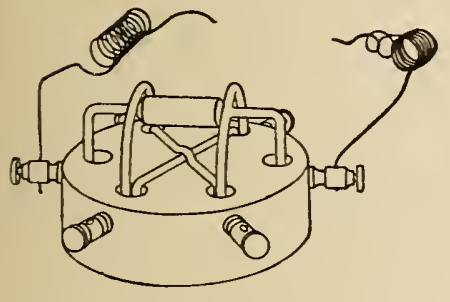

Fig. $11 \mathrm{~d}$.

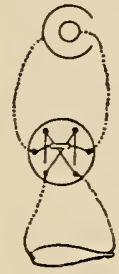

1

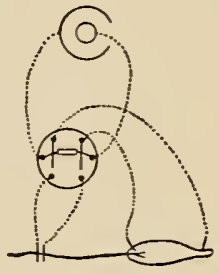

2

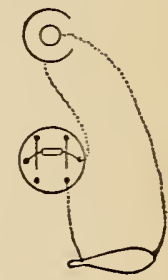

3

Fig. 11 B.

$A$, earlier form of pole-changer. The rubber handle prevents the crossing of the current from one side cup to the other. B, diagram of polechanger arranged (1) to change the direction of the current, (2) as a double key, without cross-wires, (3) as a simple key.

copper ions. It leaves the solution at the cathode where the copper ions are converted into metallic copper and deposited on the cathode. The quantity of zinc dissolved and copper deposited is proportional to the quantity of the current. One ampere deposits per minute 19.75 milligrams copper, and dissolves 20.32 milligrams zinc. 
It is to be observed that each metal is placed in a solution of its own salt. The ions carried to the respective poles are of the same nature chemically as the poles themselves, and hence do not set up opposing electromotive forces when they are de-ionized.

The current produced by the Daniell cell is almost perfectly constant, so long as sulphuric acid still remains uncombined, and so long as the sulphate of copper solution is kept saturated.

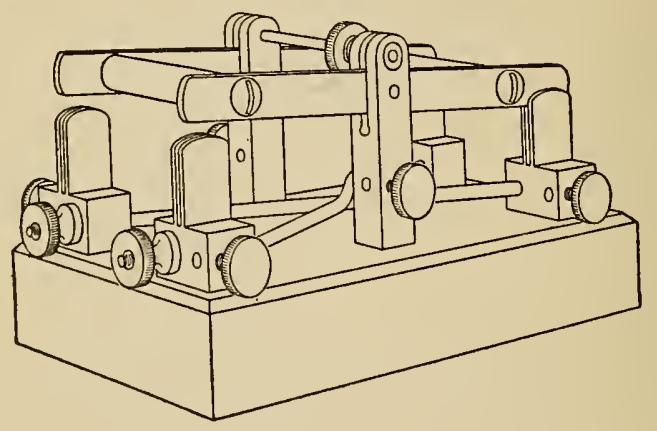

Fig. 12. Rocking key, metal contact; about one-half the actual size.

It may be remarked that the function of the porous cup is to keep the copper from depositing on the zinc.

The Pole-Changer. ${ }^{1}$ - The instrument illustrated by Fig. 12 serves as a simple key, short-circuiting key, and pole-changer. No mercury is used.

1 Science, 1905, xxi, pp. 752-754. 
The central binding posts are prolonged upwards and each is slotted to receive a brass bar, which is pivoted in the slot by a horizontal pin. The brass bars are held parallel by two rubber rods which serve as handles. When the bars are depressed to one side or the other, they engage between plates of spring brass set into brass blocks, each of which carries a binding screw. Cross-wires enter these blocks, as shown in the figure. At one end the cross-wires are soldered into the blocks, thus making an electrical contact. The two blocks at the other end are perforated by rubber cores or "bushings" through which the cross-wires pass. The cross-wires, therefore, make no electrical contact with these blocks. When a contact is desired, the nut borne on the head of each cross-wire is turned until its face presses against the brass block outside the bushing. In this position the key serves as a pole-changer, or cornmutator. When the nut on the cross-bar between the central posts is turned until its face presses against the post, it will shortcircuit the central posts.

Polarization Current. - Place two pieces of platinum foil in a solution of copper sulphate, and connect them to a pole-changer (without cross-wires). Connect the remaining pairs of posts with two dry cells in series (carbon of one cell connected with zinc of other), and with the 
0 and 1 metre posts of the rheochord, respectively. Connect the zero post and the slider to the capillary electrometer (Fig. 13). Turn the polechanger to pass the battery current through the copper sulphate solution or "electrolyte." The cation (copper) will be

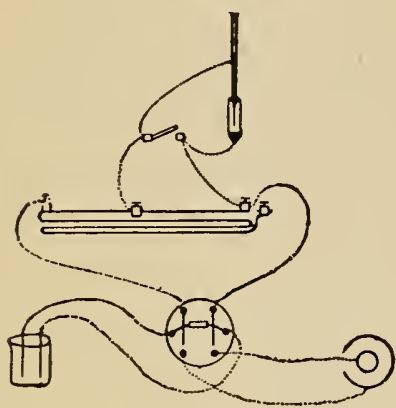

Fig. 13. partially de-ionized at the negative pole, or cathode, on which copper will be deposited in a fine film. The anion (sulphion, $\mathrm{SO}_{4}$ ) will pass towards the positive pole, or anode, where it gives up its electric charge and becomes the ordinary radical $\mathrm{SO}_{4}$. This radical cannot exist uncombined in water, but forms sulphuric acid, setting free oxygen, which therefore appears at the anode.

The elements copper and oxygen deposited respectively on the cathode and anode tend to fly back into the ionic state; and this tendency, taken in connection with the opposing osmotic force of the ions already in solution, sets up an electromotive force equal to that which caused the de-ionization, but in an opposite direction. Hence the polarization cur- 
rent. On cutting off the electrolyzing current, the polarization current may be measured.

Note the position of the meniscus of the capillary electrometer. Turn the pole-changer so that the cell is cut off and the electrodes are brought into the electrometer circuit.

The meniscus will indicate a current opposite in direction to the current from the cell.

Dry Cell. - A "dry" cell is very convenient for large classes. It usually consists of a zinc cup, lined with plaster of Paris, saturated with ammonium chloride, in the centre of which is a carbon plate surrounded with black oxide of manganese. When the cell is in action, the zincforms a double chloride of zinc and ammonium while ammonia gas and hydrogen are liberated at the carbon pole. These cells should never be used continuously for many minutes, for they are rapidly polarized by the accumulation of hydrogen on the carbon plate. The unused cell regains its difference of potential by the union of the hydrogen with the oxygen slowly given off by the manganese dioxide, which therefore acts as a depolarizer.

\section{INDUCTION CURRENTs}

A most useful method of electrical stimulation of living tissues is by the induced current, and 
a clear idea of the phenomena of induction must now be gained.

The Inductorium ${ }^{1}$ - The primary coil of the inductorium (Fig. 14), wound with double silk-covered wire of $0.82 \mathrm{~mm}$. diameter, having a resistance of 0.5

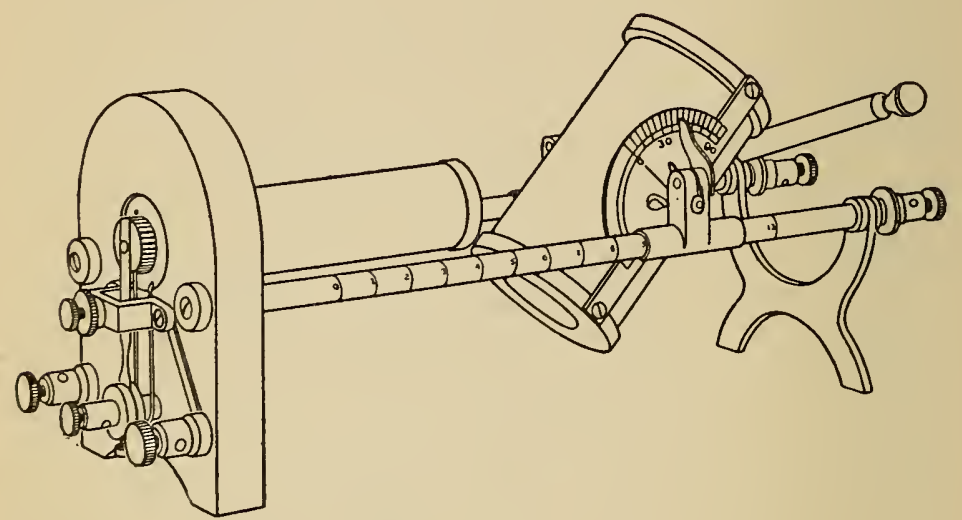

Fig. 14. The inductorium; one-third the actual size. (The set screw holding the trunnion block tube against the side rod is not shown.)

ohm, is supported in a head-piece bearing three binding posts and an automatic interrupter. The core consists of about ninety pieces of shellacked soft iron wire. This core actuates the automatic interrupter. The interrupter spring ends below in a collar with a set screw. By loosening the screw, the interrupter with its armature may be moved nearer to or

I American Journal of Physiology, 1903, p. xxxv. 
farther from the magnetic core. Once set, the interrupter will begin to vibrate as soon as the primary circuit is made. The outer binding posts are used for the tetanizing current. The left-hand outer post and the middle post are used when single induction currents are desired; they connect directly with the ends of the primary wire, thus excluding the interrupter. These several connections upon the head-piece are simply arranged and are all in view; there are no concealed wires.

From the head-piece extend two parallel rods 22 $\mathrm{cm}$. in length, between which slides the secondary coil, containing 5000 turns of silk-covered wire $0.2 \mathrm{~mm}$. in diameter. Over each layer of wire upon the secondary spool is placed a sheet of insulating paper. Each end of the secondary wire is fastened to a brass bar screwed to the ends of the hard-rubber spool.

The brass bars bear a trunnion which revolves in a split brass block, the friction of which is regulated by a screw. The trunnion block is cast in one piece with a tube $3 \mathrm{~cm}$. in length, which slides upon the side rods. A set screw, not shown in Fig. 14, holds the trunnion block tube and the secondary spool at any desired point upon the side rods. This screw also serves to make the electrical contact between the trunnion block tube and the side rod more perfect. The secondary spool revolves between the side rods in a vertical plane. When the secondary coil has revolved through $90^{\circ}$, a pin upon the side bar of the secondary coil strikes against the trunnion block 
and prevents further movement in that direction. The right-hand side bar bears a half-circle graduated upon one side from $0^{\circ}$ to $90^{\circ}$. An index pointer is fastened upon the trunnion block. One side rod is graduated in centimetres.

The side rods end in the secondary binding posts, so that moving the secondary coil does not drag the electrodes. Next the binding posts is placed a shortcircuiting key.

Magnetic Induction. - Faraday's experiment. Remove the secondary (larger) coil of the inductorium (Fig. 14) from its slideway and connect its terminals with the capillary electrometer. Raise the brass bridge between the binding posts. (If this bridge is down its thick metallic mass will offer such an easy path between the ends of the secondary wire that nearly all - practically all - the electricity produced in this coil will pass over the bridge, instead of by the relatively long, thin wires leading to the electrometer.) Bring the meniscus into the field. Thrust the north pole of a magnetized rod within the coil.

The meniscus will move, indicating that an electric current has been induced in the secondary coil. Note the direction of the current.

Let the magnet remain in the coil.

The meniscus will return to its former position. 
Evidently the induced current is of momentary duration.

Withdraw the magnet quickly.

The meniscus will move in the opposite direction.

Insert the south pole.

The induced current now has the direction opposite to that of the current induced by the insertion of the north pole.

Withdraw the magnet quickly.

The induced current has the direction opposite to that of the current induced by the withdrawal of the north pole.

These results may be thus expressed: the moving of a magnet in the neighborhood of a conductor, or of a conductor in the neighborhood of a magnet, produces in the conductor an electromotive force, which, on the circuit being completed, creates a current that would impart to the magnet or the conductor a movement in the opposite direction.

Magnetic Field. Lines of Force. - The space about a magnet in which the magnetic forces act is called the "field" of the magnet. If very fine iron filings are dusted through a muslin cloth onto a thin card perforated near the centre by a copper wire or other conductor, and a strong current is passed through the wire, the filings will 
arrange themselves in concentric circles around the wire, particularly if the card be gently tapped.

The position of these "lines of force" shows the direction of the magnetic force, and their number is an index of its intensity.

To produce Electric Induction, the Lines of Magnetic Force must be cut by the Circuit. Hold the magnet at right angles to the axis of the coil, and, keeping it in this position, rapidly advance it towards the coil.

The electrometer will show no current, because the number of the lines of magnetic force which pass through the field of the conductor has not been altered.

Electro-magnetic Induction. - An electro-magnet may be used in place of the bar magnet to produce induction.

Connect a dry cell through a simple key with posts 1 and 2 of the primary coil. ${ }^{1}$ Close the key.

When the current passes through the primary coil, the core of iron wire in the coil will be

1 It will be convenient to use the numbers 1 and 2 to designate the posts connected direetly with the ends of the primary wire, excluding the vibrating hammer; the numbers 2 and 3 will indicate the posts that eonnect with the ends of the primary wire including the hammer. When the battery is conneeted with posts 2 and 3 the hammer will vibrate. 
magnetized, as is shown by its attracting the head of the Wagner hammer.

Bring the meniscus into the field. Approach the primary coil to the secondary as in the experiment with the magnet. Withdraw the primary coil.

The electrometer shows the presence of induced currents, as before. These currents are momentary. The first induction current is inverse, $i$. e. it runs round the secondary coil in the direction opposite to that taken by the battery current in the primary coil. The second induced current is in the same direction as the primary current.

Place the coils at right angles to each other. Approach one towards the other.

No current will be induced.

Make and break Induction. - Close and open the key in the primary circuit, thus making and breaking the primary current.

The effect is the same as if the primary. were suddenly brought up to the secondary coil from an infinite distance and remored again. The make induction current is in the opposite, the break in the same, direction as the primary current.

Turn the secondary coil on its pivot until the axis is at right angles to the axis of the primary coil. Make and break the primary current.

No induction will take place provided the angle between the coils is precisely $90^{\circ}$. 
On the Construction of the Inductorium. - Examine the construction of the inductorium. The primary coil consists of a few turns of thick wire. More turns would increase resistance and selfinduction, - the counter induction set up in each turn of the primary wire by the passage of the primary current through neighboring turns, without increasing the induction effect in the secondary coil.

The iron core adds to the number of lines of magnetic induction which pass through the coils. It has been already shown (page 58) that the lines of magnetic induction produced by the passage of an electric current through a wire are closed circles. If the centre of the coil were filled with air, most of these circles would remain closed about their own wire, for air is not readily permeable to magnetism. But when the iron core is placed within the coil the greater part of the magnetic induction follows the iron (because it is more permeable) from end to end of the core, returning outside through the air. Thus the number of effective lines is increased. A bundle of iron wires is used instead of a solid core, because no induced current is then possible through the mass of the iron, as would be the case in a solid core. Such a current would slow the speed of magnetization and demagnetization. 
The secondary coil is made of many turns of fine wire, because the object of the inductorium is to transform the low electromotive force of the cell into the high electromotive force of the induced current. In the induction coil, as in other transformers, the electromotive forces in the primary circuit are to those produced in the secondary circuit approximately as the number of turns of wire in the primary is to the number in the secondary circuit.

If the induced current is to be passed through conductors of low resistance, the high internal resistance of the secondary coil, due to its great length of fine wire, will be of importance.

Place a dry cell with simple key in the pri. mary circuit of an inductorium (posts 1 and 2). Connect the secondary coil with a galvanometer. Note the excursion of the needle with a break induction current. Replace the secondary coil with one of fewer windings (the primary coil of a second inductorium will serve). Let the distance between primary and secondary coil be the same as before.

The excursion of the needle with a break induction current will be increased, or at least not proportionately diminished.

If, on the other hand, the induced current is to be passed through nerve, muscle, or skin, the 
resistance of the secondary coil will practically be nothing in comparison with the enormous resistance of animal tissue.

Repeat the preceding experiment, introducing in the secondary circuit a high external resistgnce, $i$. $e$. a nerve.

The secondary coil with many turns of fine wire now causes a much greater deflection of the galvanometer needle than the coil with fewer turns.

Interrupter. - Instead of making and breaking the primary circuit by hand, an automatic interrupter is provided. The primary circuit passes through a screw, the point of which conveys the current through a flat spring upon which is mounted an iron disk opposite and near to the core of wire in the primary coil. When the current enters the primary coil, the core is magnetized and draws upon the iron disk. The spring, to which the disk is attached, is thereby drawn away from the screw-point through which the current is passing. Thus the current is broken, and ceases to flow through the primary coil; the core no longer is magnetized, and releases the iron disk; the spring again makes contact with the screw-point, the current is reestablished, only to be at once again broken. Thus a rapid series of make and break induction currents is secured. 
Draw a diagram of the primary circuit, indicating the connections of the inductorium.

Empirical Graduation of Inductorium. - Connect the secondary coil with the galvanometer. Join the primary coil to a dry cell, interposing a simple key. Turn the secondary coil on its pivot until it is at right angles with the primary coil. Close the circuit.

The galvanometer needle will not swing. There is no induced current. ${ }^{1}$

Turn the secondary coil on its pivot, closing the key from time to time to test the induction.

The strength of the induction increases approximately as the cosine of the angle between the coils increases. An empirical graduation is sometimes placed on a circular scale beneath the coil.

When the axes of the two coils lie in the same plane, slide the secondary towards the primary, making and breaking the primary current from time to time.

The potential of the primary upon the secondary coil, $i$. $e$. the sum of the inductions of each element of the primary upon all the elements of the secondary coil, increases as the secondary is brought nearer the primary coil. The increase is not linear. As the distance between the coils

1 It is clifficult to place the coil precisely at an angle of $900^{\circ}$ 
diminishes, the increment of increase in the intensity of the induced current is not the same but greater for each centimetre of approach.

Graduation. - Fasten a strip of white gummed paper at the side of the base of the inductorium, beginning at the end block which holds the primary coil. Place the secondary coil at the end of the slideway. Make the primary current. Read the number of degrees of deviation for the break induction current only. Make a line on the paper band exactly opposite that end of the secondary coil which is nearer the primary. When the needle is again at rest, move the secondary nearer the primary coil, and find the distance at which the deviation of the needle in response to the break induction current is $n$ degrees (for example, two) of the scale larger than at the former position of the coil. Mark on the white strip the new position of the coil. Continue in this way to find the positions of the secondary coil at which the needle shows successively a deviation two degrees greater at each new position, and mark them on the paper band.

The marks on this empirical scale will be nearer together as the secondary approaches the primary coil. ${ }^{1}$

1 The rough method here employed serves merely to show that the increase in the intensity of the indnetion current as 
The Platinum Electrodes. - The stimulating electrodes are provided with platinum points projecting about $10 \mathrm{~mm}$., polished hard-rubber handle, $7.5 \mathrm{~cm}$. long, and very flexible silk-covered connecting wires $65 \mathrm{~cm}$. long, ending in nickel-plated brass tips (Fig. 15). The rubber handle is in two pieces, screwed together, permitting easy' access to the connection between the flexible wire and the stiff wire into which the platinum points are inserted.

The Flat-jawed Clamp. - The flat-jawed clamp, or "Femur Clainp" (Fig. 16), consists of strong, smoothly working brass jaws attached to a steel rod. The jaws are separated by a spring and brought together by a screw. They will hold objects of widely varying size, - for example, the femur of a nerve-muscle prepara-

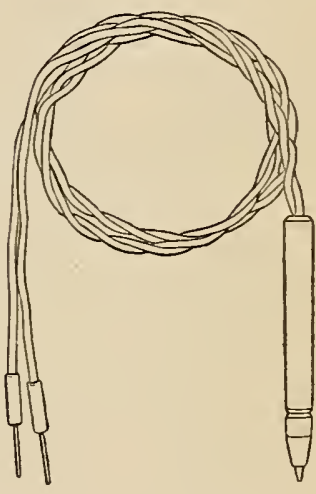

Fig. 15. The "platinum" electrodes; about one-third the actual size. tion or a board a centimetre thick. The clamp has a binding post for making electrical connection with a muscle or other conductor held between its jaws.

The Round-jawed Clamp.- The round-jawed clamp is convenient for holding burettes, tubing, rods, thermometers, etc. (Fig. 16).

The Double Clamp. - This is a strong clamp of enamelled iron with two brass nickelled screws (Fig. the coils approach is not linear. An exact method of graduation has been given by Kronecker. 
17). The screws move into an angle, against the sides of which a large or small rod may be held firmly and without sidelash.

Make and Break Induction Currents as Stimuli. - Make a nerve-muscle preparation. Connect a dry cell with simple key to the primary coil (posts 1 and 2). Fasten in the posts of the
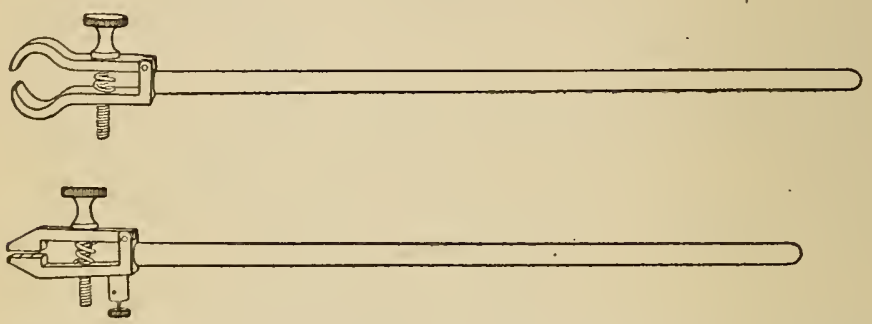

Fig. 16. The Hat-jawed clamp and the round-jawed clamp; one-fourth the actual size.

secondary coil the stimulation electrodes, $i$. $e$. the prolongation of the ends of the secondary wire which convenience demands. Put the secondary coil at the end of the slideway and turn the coil. Place the

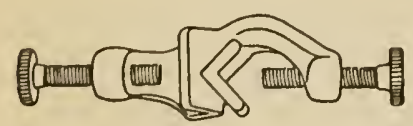

I'ig. 17. The double clamj, oncthird the actual size. electrode points against the nerve. Open and close the primary circuit.

The muscle does not contract.

Move the secondary towards the primary coil, opening and closing the primary cireuit. 
Presently the muscle will shorten. (Compare pages 175 and 176.) Observe that this contraction was the result of a break induction current, not a make.

Cautiously move the secondary coil still nearer the primary, making and breaking the current as before.

A point will be reached at which the make induction also causes contraction. Obviously, the break current is a stronger stimulus than the make induction current. The cause of the greater intensity of the break induction current lies in the primary coil. The current which enters the primary coil induces a current in this coil as well as in the secondary coil. The direction of this "self-induced" current is opposite to that of the primary current, and hence weakens it and delays its development. The stimulating power of electricity increases with both the intensity of the current and the quickness with which the intensity alters. Hence the stimulating power of the make induction current is lessened by the self-induction of the primary coil. When, on the other hand, the primary circuit is broken, the current stops, and although self-induction again takes place, it cannot affect the primary current, because the latter no longer exists. The self-induced current at the break of the primary 
current is in the same direction as the primary current before the break.

The Extra Currents at the Opening and Closing of the Primary Current. - 1. Remove the secondary coil from the inductorium. Connect posts 1 and 2 of the primary coil with a dry cell, interposing a simple key. Fasten the ends of the

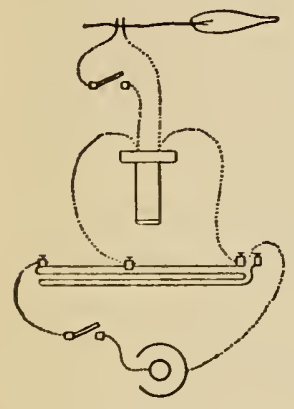

Fig. 18. electrode wires in these same posts. Close the primary circuit. Place the electrode points against the tongue. Open the key.

A shock from the selfinduced current developed in the primary coil will be felt. Draw a diagram of the circuits.

2. Connect a dry cell through a key to the metre posts of the rheochord (Fig. 18). Connect the positive post and the slider to the primary coil of an inductorium arranged for single induction currents. Bring wires from these posts of the primary coil through a simple key to the nerve of a nerve-muscle preparation. Close the key in the primary circuit. Open and close the key in the nerve circuit. The muscle will contract at closure and possibly at opening. By means of the slider, weaken the current through the primary coil until opening and closing the 
key to the nerve no longer produces contraction. Now let this key remain closed and make and break the primary circuit.

The muscle will contract both on opening and closure. The induction currents developed in the primary coil when the primary current is made and broken stimulate the nerve, although the galvanic current itself is powerless to do so.

Tetanizing Currents. - Connect a dry cell to posts 2 and 3 of the primary coil. The vibrating hammer will automatically make and break the current. Place the electrodes against the nerve or muscle.

The muscle will contract once for each induction current, but the contractions are so rapid that they fuse into a prolonged shortening termed tetanus.

Induction in Nerves. - Faraday discovered that currents can be induced in electrolytes as well as in metallic conductors. Induced currents may therefore appear in nerves lying sufficiently near a primary circuit.

Lay the well-moistened nerve of a nerve-muscle preparation around the primary coil protected by a piece of paraffin paper in such a way that the free end of the nerve touches the nerve near the muscle or touches the muscle itself, so as to form 
a closed circuit. Make and break the primary current.

Make and break currents will be induced in the nerve, and the muscle will contract.

Exclusion of Make or Break Current. - Connect the dry cell with posts 1 and 2, interposing a key. See that the short-circuiting key, i.e. the thick brass bridge between the posts on the secondary coil, is down. Connect the electrodes with the secondary coil, and place their points against the nerve of a nerve-muscle preparation. Close the primary key.

The muscle will not contract.

The resistance to the passage of the induced current through the portion of nerve between the ends of the electrodes is many thousand times greater than the resistance of the brass bridge or short-circuiting key. Practically none of the electricity will pass through the nerve when the short-circuiting key is closed.

Open the short-circuiting key and then open the primary key.

The muscle contracts.

Repeat the experiment, letting the make current pass and short-circuiting the break.

With the primary key and a short-circuiting key either break or make induced currents can be used as stimuli at will. 


\section{UNIPOLAR INDUCTION}

1. Arrange the inductorium for tetanizing currents (posts 2 and 3). Make a nerve-muscle preparation. Lay it on a clean dry glass plate. Let the nerve rest on a wire connected with one pole of the secondary coil. Set the inductorium in action. Connect the muscle with the earth by touching the muscle with the end of $x$ wire the other end of which rests on a gas or water pipe.

The muscle will show tetanic contractions, provided the induced current is sufficiently strong. If no tetanus is seen, move the secondary coil completely over the primary.

Unipolar induction may be produced by the electric currents in the skin. This may be demonstrated with a sensitive nerve-muscle preparation.

2. Ligature the nerve between the electrode and the muscle, and repeat the experiment.

Stimulation will still be secured. The unipolar discharge passes through the entire length of nerve and muscle to or from the point at which the connection with the earth is made, and thus stimulates the entire preparation.

DuBois-Reymond, who was the first to make the preceding experiments, pointed out that 
whenever the secondary gircuit was open (i. $e$. when the bridge between the ends of the secondary wire was up) the making and breaking of the primary circuit caused free electricity to gather on the ends of the secondary wire. When the electro-static induction becomes great enough the electromotive force overcomes the resistance in whatever connecting path may be offered, and the electricity passes from the coil to the earth. If a part of the path is formed by irritable tissues, they will of course be stimulated.

3. The quantity of electricity passing through the nerve may be increased by approximating the coils or by increasing the electrical capacity of the conductor, as follows:-

Remove the wire connecting the preparation with the gas pipe. Set the inductorium in action. Touch the muscle with the moistened finger.

Contraction follows.

Here the electrical capacity of the preparation is increased by connecting the preparation with the human body, a conductor of large surface (and through it with the earth). A similar result is obtained by unipolar stimulation of nerves and muscles while still in the body of the animal, as in many physiological experiments. It is not necessary that the surface of the conductor be enormously large. The follow- 
ing experiment shows that even very small surfaces will suffice.

4. On a carefully dried, clean glass plate lay four nerve-muscle preparations. Let the nerve of the first rest on a single wire the other end of which is fastened in one of the binding posts of the secondary coil. Place the end of the second nerve on the tendon of the muscle of the first preparation, the third on the second tendon, and the fourth nerve on the tendon of the third. Remove the secondary coil some distance (a few centimetres) from the primary, and set the inductorium in action. Gradually approximate the coils.

As the tension at the ends of the secondary wire increases by the approximation of the coils, the first preparation will contract. On further approximation, the first and second; then the first, second, and third; and finally all four will contract.

This instructive experiment shows that when the conducting surface is small, as in the present instance, the unipolar action is greater on the parts nearer the secondary wire than on parts farther away. The danger of unipolar action on tissues lying near the electrodes in ordinary artificial stimulation of nerves and muscles in situ is obvious. 
5. It is not even necessary that the conductor should be actually in contact with the preparation.

Comnect a nerve-muscle preparation, insulated on a glass plate, with one pole of the secondary coil, and set the inductorium in action. The secondary coil should completely cover the primary. Bring a moistened finger as near the muscle as possible without touching it.

With the proper intensity of the primary current, contraction will take place, though absent when the finger is removed.

The sudden approach of a condenser charged with static electricity will stimulate an isolated nerve or muscle.

6. The danger of error from unipolar action is particularly great in electrometer observations on the current of rest or action current of nerve and muscle, discussed and demonstrated experimentally in Part III, Chapter II.

The errors due to unipolar action can usually be prevented by the following precautions: The secondary coil should always be connected with the tissue to be stimulated through a shortcircuiting key, which should be kept closed except during the intentional stimulation of the tissue. With this good metallic connection between the ends of the secondary wire there will 
be no static electrification. Further, the appearance of positive and negative electricity during the period of stimulation must be provided against, especially if that period is at all protracted, for it must not be forgotten that the bridge of nerve, which completes the secondary circuit by uniting the two electrodes, possesses very high resistance, and thus affords but an imperfect closure of the ends of the secondary wire. This provision is made by connecting the positive electrode with the earth by a good conductor, for example by a copper wire leading from the electrode to the gas or water pipe In case of doubt, a control experiment should be made. The nerve should be severed between the stimulated point and the muscle, and one end laid on the other. Excitation through the passage of a nerve impulse along the nerve is thereby made impossible. If the muscle still contracts when the nerve is stimulated above the section, it is because of unipolar stimulation.

An additional reason for care is that the insulation of the secondary spiral is injured by leaving the secondary circuit open while the hammer of the inductorium is in action.

It may be stated that the direction of the unipolar discharge is of importance. Excitation takes place only where the positive charge enters 
the nerve or the negative charge leaves the nerve.

The break induction current is more effective than the make, as the slower development of the latter causes the terminals of the secondary wire to be charged more slowly than by the rapidly developed break current.

\section{Apparatus.}

Normal saline. Bowl. Towel. Pipette. Glass plate. Zinc wire, 4 inches long. Copper wire, 4 inches long. Porcelain dish. Mercury. 5 per cent sulphuric acid. 5 per cent solution of potassium chromate. Iron wire, 4 inches long. Muscle clamp. Iron stand. Capillary electrometer. Rheochord. Microscope (nicrometer ocular, objective 3). Daniell cell. Dry cell. Two platinum electrodes. Zinc electrode. Beaker. Sodium chloride. Simple key. 9 wires, 2 feet long. Saturated solution of copper sulphate. Pole-changer (in paper dish). Inductorium (with electrodes). Coil with few windings (primary coil of a second inductorium). Bar magnet. Iron filings. Galvanometer. Card, with thick copper wire. Ligatures. Frogs. Osmometer (for demonstration). Tradescantia discolor. Serum. Sodium chloride solutions $(0.60,0.65$, $0.70,0.75,0.80$ and 5.0 per cent, also $0.62,0.61,0.60$, $0.59,0.58$, and 0.57 per cent). Microscope. Defibrinated hlood. Twelve test-tubes. Tension indicator. Soap solution. Lycopodium. Alcohol. 


\section{III}

\section{THE GRAPHIC METHOD}

The studies next to be undertaken make use of the change of form of the contracting muscle as a partial index to the transformation of energy in the tissue. A permanent record is desirable. Further, the changes in the dimensions of the muscle are so small that it is necessary to have the graphic record enlarged, rather than of actual size. To satisfy these conditions, the muscle is attached near the fulcrum of a lever furnished with a recording point. The surface for the writing is usually glazed paper which has been covered with a thin layer of soot by passing the paper through the luminous part of a broad gas flame. The paper is fastened (before smoking) on a plate or on a drum which moves past the writing point, almost parallel to it, and furnishes thus a continuously fresh surface. ${ }^{1}$

1 The paper is cut wider and longer than the surface of the drum. The extra width is to protect the bearings of the drum from soot that might otherwise collect there in smoking the 
The writing point rubs off the soot in its path and leaves a white magnified tracing of the muscle's change in length or whatever dimension is the subject of record. The paper is then removed, drawn through a saturated solution of

paper. The extra length allows the edge of the overlap to be gummed to the paper below, permits the paper to be removed from the drum by cutting through the overlap parallel to the mucilage, - the surface of the drum being protected from the knife by the underlying paper, - and provides an unsmoked surface by which the paper can be handled on its removal from the drum. The drum should be laid in the centre of the strip of paper, the gummed edge to the left, and the axis of the drum precisely at right angles to the long axis of the paper; the mucilage should be moistened, and the ends of the paper brought around and fastened. If the paper is awry, the surface will not lie uniformly against the drum and the record will be deformed. The drum should now be placed in the smoking apparatns, revolved uniformly and not too fast, brought over the gas flame, lowered just below the upper eige of the flame, and covered with a chocolate brown layer of soot, beginning at the operator's left hand and passing gradually to the right. The speed should be such that one passage from left to right shall suffice. To trim the edges, hold the drum in the left hand, inclined downwards, and pass a sharp knifeblade around the lower edge. The handle of the knife should be kept lower than the blade, to avoid tearing. In removing the paper from the drum, hold the drum in the air with the left thumb pressed on the edge of the paper near the overlap, and eut through the overlapping edge near the mucilage. The loosened paper will hang down and may then be seized by the unsmoked overlap. In recording, let all the curves begin near the overlap. Attention to these details is indispensable to the best technical results. 
white shellac in 95 per cent alcohol, ${ }^{1}$ and hung up until the alcohol is evaporated. The soot will thus be coated over and held in place by a thin layer of shellac, and the record will be secure.

The Kymograph. - The improved kymograph ${ }^{2}$ is shown at the right of Fig. 19, in which it is mounted as part of the long paper device. It consists of a drum revolved by clockwork and also arranged to be more rapidly revolved or "spun" by hand.

The drum is of aluminium, cast in one piece turned true in the lathe to a circumference of $50 \mathrm{~cm}$. The height is $15.5 \mathrm{~cm}$. The weight is about 600 grams. The drum slides upon a brass sleeve in bearings $1.1 \mathrm{~cm}$. deep (to prevent "sidelash"), and is held at any desired height by a spring clip. The sleeve ends in a friction plate, which rests upon a metal disk driven by the clockwork. Sleeve and friction plate revolve about a steel shaft which passes through both the heary plates containing the clockwork, and is securely bolted to the bottom plate. The sleeve bears upon the steel shaft only by means of "bushings" at the ends of the sleeve, thus securing

1 To make this solution, the alcohol should be allowed to stand on the shellac a month or more before using. A satisfactory solution may be made in twenty-four hours by dissolving 375 grams of rosin in 2500 c.c. of alcohol.

2 Introduction to Physiology, 1901, p. 51. American Journal of Physiology, 1903, viii, p. xxxvii. Ibid., 1904, x, p. xxxix. Science, 1906. 
a bearing without "sidelash" and with little friction. As the sleeve with the drum rests upon the friction plate by gravity alone, it is easy to turn the drum by hand either forward or back, even while the clockwork is in action. At the top of the sleeve is a screw ending in a point which, when the screw is down, bears upon the end of the steel shaft and lifts the sleeve, and with it the drum, until the sleeve no longer bears upon the friction plate. The drum may then be "spun" by hand about the steel shaft. The impulse given by the hand will cause the drum to revolve for about one minute. The speed during any one revolution is practically uniform.

The clockwork consists of a stout spring about 6 metres in length, driving a chain of gears. The speed is mainly determined by a fan slipped upon an extension of the last pinion shaft in the chain. Four fans of different sizes are provicled.

The speed is regulated by a governor on the shaft that carries the fan. When the milled head shown to the right of the steel shaft in Fig. 19 is up, the gear on the extreme right of the chain no longer engages with the gear driven by the spring, but runs "idle," while the gear attached to the friction plate engages with the lower of the two gears at the left; the pinion of this lower left-hand gear engages with the spring gear. Fast speeds are then obtained.

When the milled head is down, the gear attached to the friction plate falls below the left-hand gear, while the right-hand gear engages with the spring 
gear and through a pinion drives the friction-plate gear. Slow speeds are then obtained.

These operations are easily and rapidly performed, though, as in all gear mechanism, an instant's pause is sometimes required to enable the gear teeth to engage. The clockwork should be in motion, without the fan, when the adjustments are being made.

With both fast and slow gearing four fans of different areas may be used. They are slipped upon an extension of the last pinion shaft in the chain. Five slow and five fast speeds (exclusive of spinning) are thus obtained. An additional slow speed $(50 \mathrm{~cm}$. per hour) may be obtained with a very large fan. All speeds are regulated by a friction governor fastened to the same shaft that carries the fan. With one winding the drum will revolve from about one to about seven hours, or longer, depending on the fan employed.

The Long Paper Kymograph. ${ }^{1}$ - In Fig. 19 the kymograph is arranged for use with a sheet of smoked paper about eight feet long. A rigid bench of steel about 97 centimetres long firmly supports two ]-shaped castings in which two aluminium drums revolve on pointed adjustable bearings. One of the castings slides along the bench, and may be fastened at any desired distance from the remaining or clockwork drum, so that paper from about 150 to 240 centimetres in length may be stretched between the

Science, $_{2} 1906$ 
82 GENERAL PROPERTIES OF LIVING TISSUES

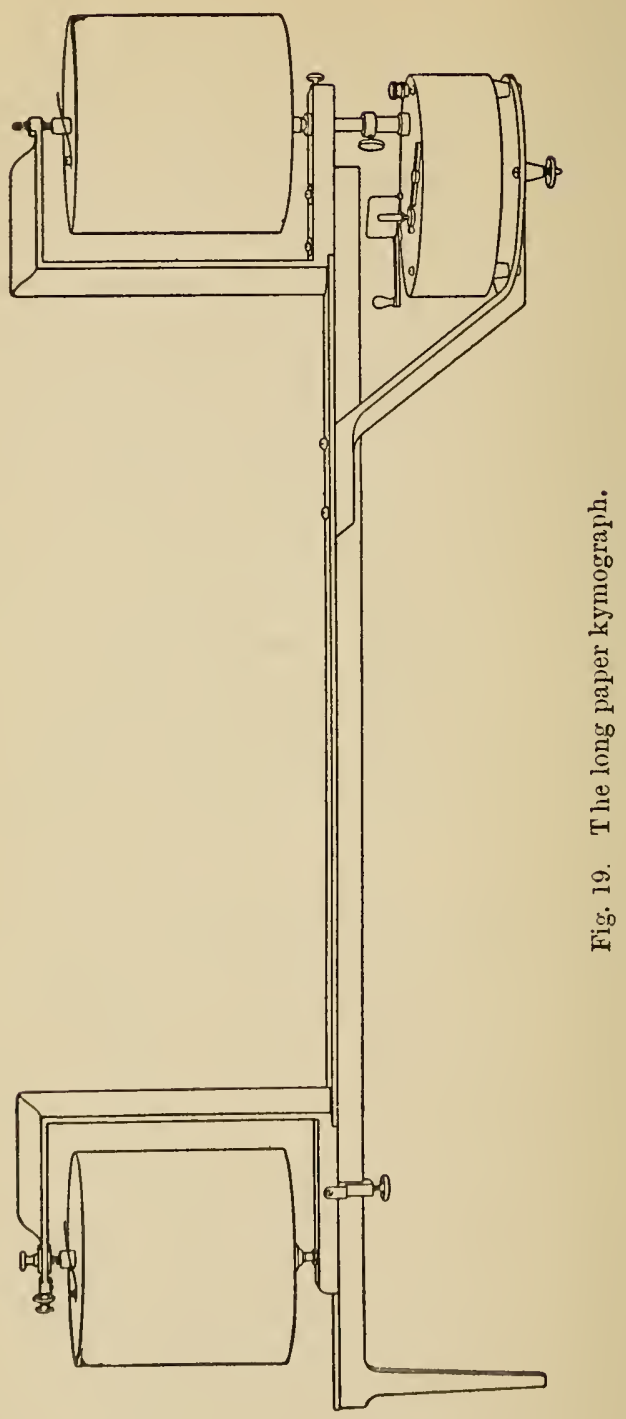


drums. Each drum is provided with an adjusting screw, by means of which the drum may be inclined until the strip of paper is stretched uniformly throughout its height. This adjustment should preferably be made upon the sliding drum. When the adjustment is complete, the abscissae drawn by a writing lever in successive revolutions will exactly coincide. The clockwork drum does not slide along the bench. Both drums may readily be removed from their bearings.

Beneath the clockwork drum is a circular plate of the exact size of that of the medium spring kymograph. This plate rests on two feet and in fact supports the anterior end of the steel bench. The clockwork drum is driven by a kymograph in which the vertical steel drum-rod and sleeve are replaced by a short rod the top of which is flush with the upper plate of the kymograph. The feet of this kymograph are hollowed to fit three rounded pins. When the kymograph is set upon these pins, it is at once "centred" and all side motion is prevented. A coupling sleeve is now let down from the shaft of the clockwork drum until two projections on the under surface of the coupler engage with corresponding slots in the kymograph rod. The clockwork operates like that of the medium-spring kymograph, having ten changes of speed. The speeds are, however, faster as a stronger spring is used, the maximum being about seven centimetres per second.

To smoke the paper, the coupler is raised, the 
kymograph clockwork is removed, and then the entire bench together with its drums is placed horizontally in the smoker frame (Fig. 20).

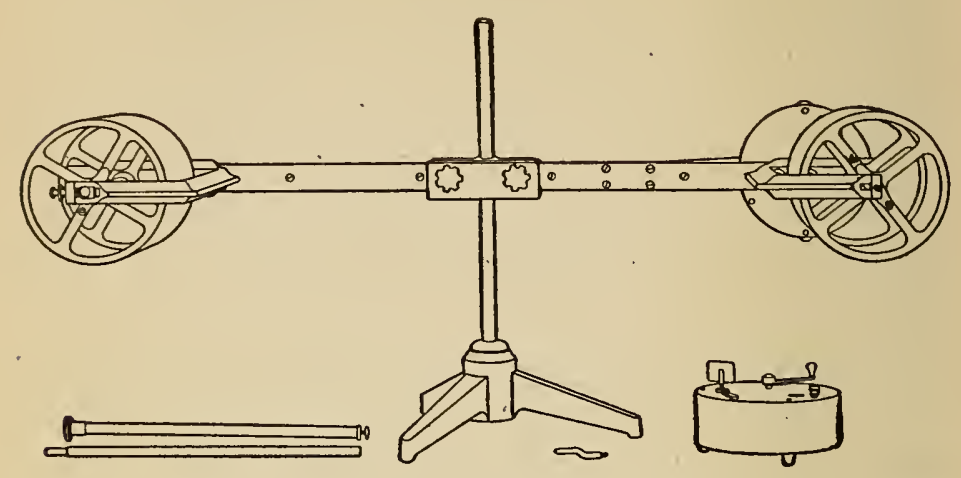

Fig. 20. The smoker, showiug the long paper kymograph in place. The paper is smoked with an oil lamp having a four inch wick. Near the stand are the handle with which the drum is revolved to carry the paper over the lamp flame, and the two rods which are inserted in the kymograph clockwork when the latter is used independently of the long pajer arrangement.

The graphic record involves the use of apparatus. It never should be forgotten that the use of apparatus always introduces more or less error. In every experiment the apparatus should be criticised sharply. The numerous imperfections which such scrutiny will bring to light are of two sorts, - the errors that may be neglected, and the errors that may not be neglected without seriously impairing the value of the method for the purpose in hand. For example, a count of the pulse rate with an ordinary watch will usually be incorrect by one or 
two beats in the minute, but such a record is quite accurate enough for most purposes. The use of a stop-watch marking fifths of seconds would add nothing to the value of the count, for the error introduced by numberless causes that slightly modify the heart-beat from minute to minute is greater than the error introduced by using an ordinary watch instead of a stop-watch. The correction of errors that are too small to alter essentially the value of the method for the purpose to which it is applied is usually wasteful.

With these points in mind, smoke a drum. Arrange the inductorium with simple key for maximal break induction currents. Prepare a gastrocnemius muscle, fasten it in the muscle clamp, tie a fine copper wire around the tendo Achillis, wrap the wire about the hook on the muscle lever, and fasten the end in the binding post of the muscle lever (Fig. 21). Connect the secondary coil with the posts on the muscle clamp and muscle lever respectively. Weight the muscle with ten grams: Arrange the lever to write on the drum. Record single contractions with various speeds.

Note that the muscle writes its contraction in the form of a curve, the ordinates of which measure the height to which the load is lifted. 
Light Muscle Lever. ${ }^{1}$ - A stout yoke (Fig. 21) bears two set screws holding a steel axle upon which is mounted a light piece of tubing and a hard-rubber pulley. One end of the tubing tapers slightly to receive the writing straw. The other projects behind the axle, and may be pressed upon by the accurately cut after-loading screw. The pulley is pierced with a hole for securing a fine wire by means of which a

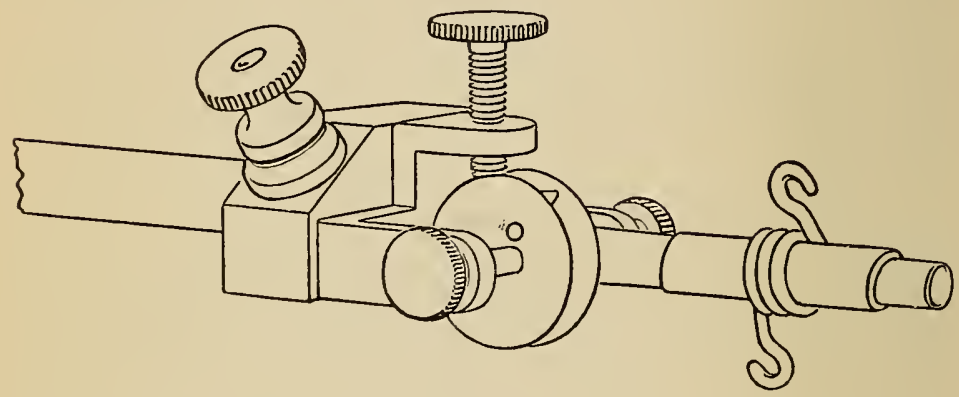

Fig. 21. The liglit muscle lever, with double hook straw fastener; the actual size.

weight may be suspended from the pulley when it is desirable that the weight should be applied near the axis of rotation. The muscle may also be weighted directly by means of a scale-pan suspended from the double hook to which the lower end of the muscle is attached. If the tendon of the muscle be fastened to the double hook by a fine wire, the free end of the wire may be carried to the insulated bind-

1 First Catalogue of Harvard Physiological Apparatus, September, 1901. 
ing post provided for convenient electrical stimulation. The upper end of the muscle may be grasped in the flat-jawed clamp (Fig. 16), and thus comnected electrically with the binding post upon it.

In obtaining the extension curve of muscle this lever, after-loaded, may be weighted to one hundred grams without bending and thus deforming the curve. The abscissa will be a straight line. The moving parts are very light. The apparatus is compact and occupies but little of the vertical space so valuable where several recording instruments must be placed upon the same stand.

Writing Lever. - A strip of aluminium, bent at one end to fasten with the double hook, pointed at the other, may be used in place of a straw.

Tuning Fork. - A nickelled polished steel fork (Fig. 22) with steel handle is filed until it gives one hundred double vibrations per second. The tuning fork may be provided with a paper or foil writing

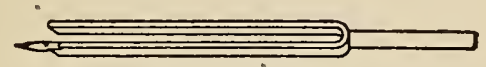

Fig. 22. The tuning fork; about one-sixth the actual size.

point and clamped to the iron stand. It serves to measure the latent period of muscular contraction and similar phenomena of brief duration.

Start the drum at very rapid speed. Bring the writing point of the vibrating tuning fork 
(Fig. 22) against the paper below the point of the muscle lever, and stimulate the muscle to contract.

Observe that the tuning fork now gives the time intervals on the abscissa of the muscle curve, from which the duration of the periods of shortening and relaxation may be known. Note also the difference in appearance of curves recorded on a slow and a rapidly moving surface.

Measure the interval between the beginning of contraction and the point of maximum shortening.

In your laboratory note-book write a critical account of the muscle lever.

Compare this account with the remarks which follow :-

The object of the muscle lever is to write a magnified record of the change in form of the muscle. Usually the muscle is suspended in a muscle clamp and its lower end attached to the lever, which then records the shortening of the muscle. The same lever may be used to record the thickening of the muscle; in this case the muscle is of course horizontal and the lever rests upon it. For either purpose the weight of the lever is an objection, for it tends to prevent the muscle from begin- 
ning its movement (inertia of position). Once in motion, the weight tends to keep moving, and thus to continue the record of contraction after the actual contraction has ceased (inertia of motion). As the inertia of motion increases with the mass and the square of the velocity, the lighter the lever the less the error. The disposition of the weight relative to the axis is also of importance. In a swinging system, the nearer the mass to the axis of rotation, the less are the after vibrations or pendulum-like oscillations which continue after the original impulse has ceased. For this reason, in experiments likely to be disturbed by after vibrations, the weight which the muscle lifts is attached to the small pulley, so as to be as near the axis as possible. In this case, the weight on the muscle is of course not the weight hung on the pulley; the pulley weight must be divided by the number of times the radius of the pulley is contained in the distance between the axis and the point of attachment of the muscle to the lever.

It will be observed that the writing point is a strip of tinsel bent slightly and placed parallel to the writing surface. It is very easily moved in a direction at right angles to the writing surface, but resists movement in a vertical direction. 
The bend makes the strip a weak spring, enabling the point to remain in contact with the drum throughout the excursion of the point on the paper. The writing point should be as nearly as possible parallel to the paper. Even in this position, the distance of the end of the straw from the paper is necessarily less when the lever is horizontal than when raised by the contraction of the muscle, for the end of the lever describes a curved line in a plane tangent to the recording surface. Were it not for the spring of the writing point, the latter would leave the drum. To remain on the drum at the height of the contraction, the point must at the beginning of contraction press against the drum with much more friction than is necessary simply for scratching through the layer of soot. Thus the distance of the writing point from the axis is constantly varying, and the magnification of the lever is constantly changing. Within the limits ordinarily employed in physiology, the deformation of the curve thereby produced is proportional to the length of the are through which the point moves; the curve should therefore be written no larger than is necessary for clearness.

When the smoked surface is at rest, and the contracting muscle lifts the lever, the writing point describes an arc; when the muscle relaxes, 
the writing point returns in the same line. When the drum revolves, the writing point describes a curve as the muscle contracts. The maximum shortening of the muscle, or height to which the load is lifted, is measured by a perpendicular drawn from the highest point of the curve to the abscissa. The time required for the muscle to reach this height, however, is not the distance on the abscissa from the beginning of the curve to the perpendicular, but to the point at which the segment of a circle of a radius equal to the length of the lever would cut the abscissa when drawn from the highest point of the curve. Practically, this measurement is made by turning the drum back until the point of the raised lever rests at the summit of the curve, and then, while the drum is at rest, allowing the lever to write the ordinate by falling down to the abscissa.

Perpendicular ordinates may be secured by a long pin passed transversely through the end of the writing lever, and bent twice at right angles, first parallel to the paper and then towards it. The lever is perpendicular to the paper and very near it; the weight of the pin keeps the point against the paper as the lever rises. The perpendicular writing has many faults in common with are writing. 
92 GENERAL PROPERTIES OF LIVING TISSUES

\section{Apparatus}

Normal saline. Bowl. Pipette. Towel. Glass plate. Kymograph. Glazed paper. Smoking apparatus. Shellacking trough. Shellac in alcohol. Muscle lever (weight pan). Muscle clamp. Stand. Inductorium. Electrodes. Simple key. Dry cell. 5 Wires. Fine copper wire. Ten gram weight. Tuning fork. Tin foil. Cement. Frogs. 
THE ELECTRICAL STIMULATION OF MUSCLE - AND NERVE

\section{The Galtanic Current}

THE study of the changes occasioned in muscle and nerve by electrical stimulation may profitably begin with the action of the galvanic current.

Non-Polarizable Electrodes. - When metal electrodes come in contact with an electrolyte, polarization currents develop (see page 51). Electrodes of metal for this reason should be avoided in the study of the effect of the galvanic current on muscle and nerve. A "non-polarizable" electrode should be employed. Strictly speaking, no electrode is non-polarizable, but practically the polarization errors are excluded by the device shown in Fig. 23.

The boot electrodes (Fig. 23) are made of potter's clay, skilfully fired, and are unglazed. The leg is pierced with a hole $28 \mathrm{~mm}$. deep and $8 \mathrm{~mm}$. in 


\section{9t GENERAL PROPERTIES OF LIVING TISSUES}

diameter, in which is placed the zinc. The foot is $20 \mathrm{~mm}$. long, measured from its junction with the leg. In the foot is a well for normal saline solution which shall keep the feet equally saturated. The boots should ordinarily be kept in normal saline solution. In use the hollow leg of the boot is half-filled with saturated solution of zine sulphate and placed in the clip. The well in the foot of the boot is now filled with normal saline solution. If metal clips are used

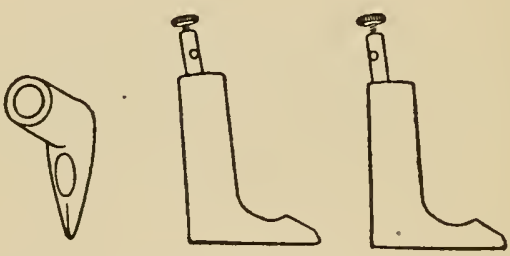

Fig. 23. Non-polarizable electrodes; about two-fifths the aetual size. 1

the boots should be mounted on separate rods, to prevent the current passing through the unglazed boot to the metal holder and thus to the other boot. This difficulty is avoided by the rubber holders shown in Fig. 24. The electrodes may be mounted on a brass rod ealled the mounting-rod, or in the moist chamber shown in Fig. 24. The boot electrodes serve equally well for leading off the nerve or mus-

1 First described in "Science," 1901, xiv, pp. 567-570. The well was added in Nov. 1905. 
cle current to the electrometer and for stimulation. After use, the boots should be emptied, rinsed in tap water, drained, and placed in several hundred cubic centimetres of normal saline solution until wanted again. If the foot of the boot is liept saturaterl with normal saline solution these electrudes will remain

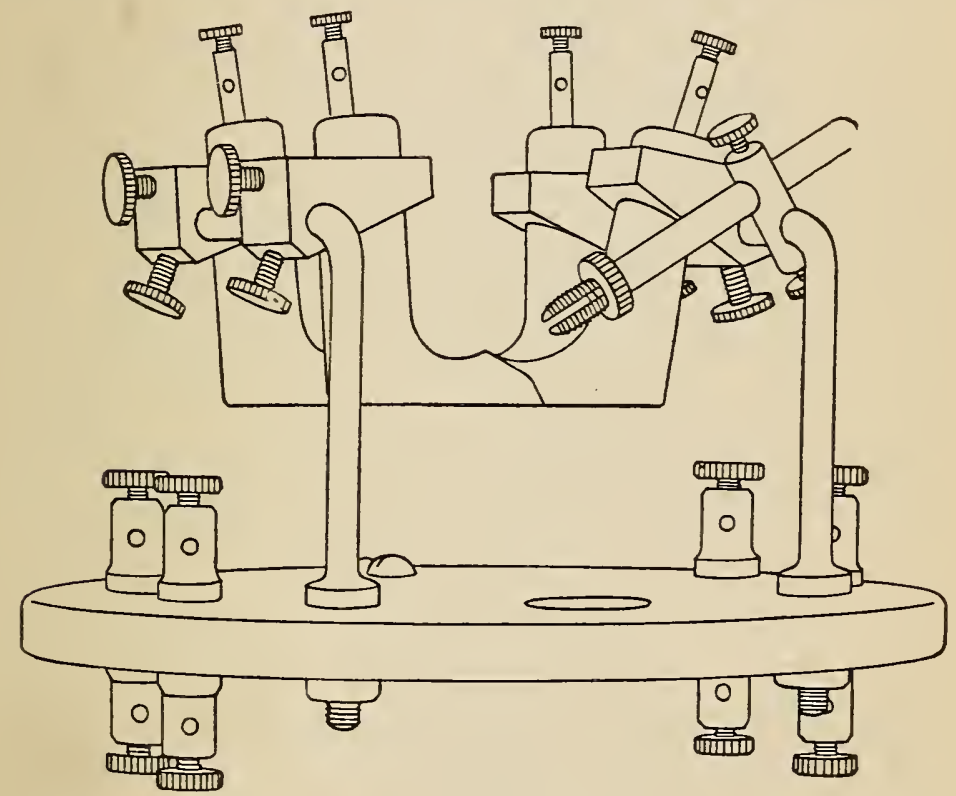

Fig. 24. The moist chamber; about three-fifths the actual size.

non-polarizable. They may also be used with normal saline clay.

The Moist Chamber. ${ }^{1}$ - The moist chamber (Fig. 24) consists of a porcelain plate which bears near

1 Science, 1901, n. s. xiv, p. 569. 
the margin a shallow groove. In this groove rests a glass cover which for the sake of clearness has been omitted from the figure. To the porcelain plate is screwed a rod, by which the plate may be supported on a stand. Within the glass cover are two rightangled rods. One of the rods carries a small clamp, composed of a split screw on which moves a nut, by means of which the femur of a nerve-muscle preparation may be firmly grasped. The holder for the split screw is arranged to permit of motion in all directions. Both right-angled rods carry umpolarizable electrodes. Each of these is borne by a hard-rubber holder. By turning the leg of the boot in the holder the foot may be brought as near the foot of the neighboring electrode as may be desired. It is desirable to mount the boots on opposite rods as in Fig. 24. A thick wire of freshly amalganated zinc, provided at one end with a hole in which a connecting wire may be fastened with a set screw, is placed in the leg of the boot, and the other end of the connecting wire brought to one of the four binding posts shown in Fig. 24. These four posts are in electrical connection with four other posts beneath the porcelain plate. The air within the noist chamber may be kept saturated with water vapor by applying moist filter paper to the inner side of the glass globe.

Destruction of the Brain by Pithing. - The next experiment requires a curarized muscle, and 
curarization is best accomplished by the injection of curare into the dorsal lymph sac of a frog the brain of which has been destroyed. Wrap the frog in the cloth, head out. Hold the frog with the fingers of the left hand, pressing down the tip of the frog's nose with the left thumb. Pass the right forefinger along the middle line of the head. A slight depression will be felt at the joining of the skull and trunk. Here the cerebro-spinal canal has no bony covering. Make at this point a cut about a centimetre $\left(\frac{2}{5}\right.$ inch) long through the skin in the middle line. Thrust the seeker vertically through the soft tissues until the point is stopped by the bony vertebrae. Turn the point of the seeker towards the head, and push it along the brain cavity, moving it gently from side to side.

Paralysis of Voluntary Motion by Curare. Make a very small hole in the skin of the back into the dorsal lymph sac. With a fine glass pipette inject a few drops of a straw-colored solution of curare. The curare of commerce is the dried juice of a species of strychnos. It is not a definite chemical compound and cannot therefore be given in an accurate dose. It is customary to make a one per cent solution of the crude mass. This solution may be kept from 
decomposition by a small crystal of thymol. The bottle should be shaken before the curare is withdrawn. The curare should be injected at the begimning of the laboratory day, so that there may be time for its action in a dilute solution. The motor nerves are paralyzed first and the effect should, if possible, be limited to them. Strong solutions paralyze other nerves, the heart, and probably other muscles.

Opening and closing Contraction. - Place two non-polarizable boot electrodes in rubber holders upon a mounting-rod. Fill the boots half full of saturated solution of zinc sulphate. Fill the well in the toe of each boot with normal saline solution. Place well amalgamated zincs in the boots and connect them through an open simple key with the poles of a battery. Prepare a sartorius muscle (Fig. 25) from a curarized frog, ${ }^{1}$ preserving the pelvic and tibial attachments. Lay the muscle upon the toes of the boot electrodes. Close the key.

The muscle will twitch when the current is made and probably when it is broken, but during the passage of the current there will be normally no contraction.

1 Be sure to cut off the head or otherwise destroy the brain of curarized frogs before operating on then. 
In frogs used during the period of hibernation and especially in those brought from a cold storeroom into a warm laboratory, the make and sometimes the break of the constant current may be followed by prolonged tetanus. In such frogs, the irritability of the muscles is greatly increased, and the changes, probably ionic, which occur while the current is passing and after it is shut off are sufficient to produce contractions not seen in the normal state (see page 147). Usually the muscle is stimulated only by a sudden change in the intensity of the current.

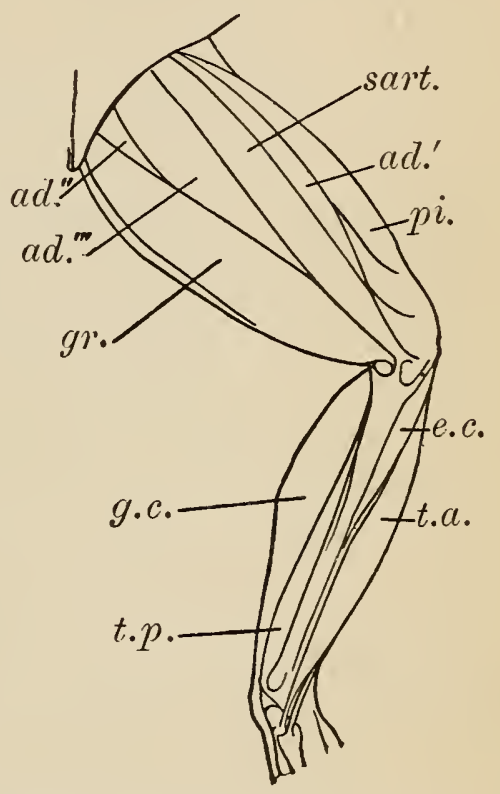

Fig. 25. Hind limb of frog, anterior view (Ecker-Wiedersheim).

\section{Changes in Intensity of Stimulus. - Connect} one of the electrodes used in the preceding experiment with one of the poles of a dry cell. From the other pole lead a wire to a bowl of salt water. To the other side of the same bowl bring a wire from the remaining electrode.

When the wires are slowly brought nearer 
together, there will be no contraction; when they are brought quickly together, thus quickly increasing the intensity of the current, the muscle will contract.

With Indirect Stimulation. - 1. Smoke a drum. Make a nerve-muscle preparation (sciatic nerve and gastrocnemius muscle). Place the femur in the clamp in the moist chamber. Let the nerve rest on non-polarizable electrodes connected through an open key with a dry cell. Attach the tendo Achillis to the muscle lever. Let the muscle lever write on a slowly moving drum. Close and open the key.

Both closing and opening contraction will be seen. (If the frog has been brought from a cold room into the warm laboratory, opening and closing tetanus will probably replace the usual twitch. See page 147.)

2. Repeat the experiment on page 99 , using the nerve-muscle preparation instead of the curarized muscle.

It will again be found that the intensity of the current must be increased with a certain rapidity in order to stimulate.

The experiments just made support DuBoisReymond's statement that the electrical current does not stimulate during the entire period of its flow through the irritable tissue, but only 
when the intensity is rapidly altered by making or breaking the circuit. These experiments, however, were made on the rapidly reacting skeletal muscle of the frog. The law does not hold good for sluggish contractile tissue. Indeed it can be disproved even for highly striated muscle by a very careful examination of the manner in which excitation takes place. Pfliger discovered that when the galvanic current is made, excitation takes place only at the points through which the current leaves the muscle or nerve (cathodal stimulation), and that when the current is broken, excitation takes place only where the current enters the irritable tissue. This "polar excitation" we must now consider. We shall find, among many other facts, the refutation of the idea that stimulation does not occur throughout the passage of the current.

\section{Polar Stimulation of Muscle}

1. Slit the curarized sartorius muscle trouserlike from the lower end. Lay each end on a boot electrode. Make and break the current.

On making the current the cathodal side will contract; on breaking, the anodal side.

2. Lay the muscle on ice covered with a small piece of paraffin paper, to shield the muscle from 
water. When thoroughly cold, place the muscle in the Gaskell clamp (Fig. 26), making very gentle pressure across the middle, and bring the non-polarizable electrodes against the ends. Make and, after a minute, break the current.

The excitation wave passes so slowly through cooled muscle that the contraction can be seen with the unaided eye to begin at the cathode on closing and at the anode on opening the circuit.

3. Ureter. ${ }^{1}$ - Place the extirpated ureter of any mammal on a glass plate set as a cover on a beaker containing hot normal saline solution, so that the hot vapor of the water shall keep the ureter warm. Bring the non-polarizable electrodes against the ureter. Note which electrode is the cathode. Close the key.

After a distinct latent period the ureter in the cathodal region, and nowhere else, will contract, and the contraction wave will spread from the cathode in both directions along the ureter.

Open the key.

The Gaskell Clamp. - The tapered edge of a hardrubber block is brought against a similar edge by means of a fine screw (Fig. 26). With this clamp the heart muscle may be compressed, after Gaskell's

1 The experiment succeeds also with extirpaterl pieces of intestine abont four inches long, provided they are kept warn with normal saline solution. 
STIMULATION OF MUSCLE AND NERVE 103

method, until conduction between auricle and ventricle is partially or wholly interrupted. The clamp

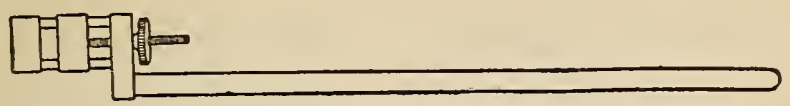

Fig. 26. The Gaskell clamp ; about one-third the actual size. ${ }^{1}$

is also used to press upon the sartorius until the conduction wave is blocked while the excitation wave still passes.

The contraction takes place now only at the anode, and the contraction wave spreads from that point over the muscle (as making the current is a less effective stimulus than breaking it may be necessary to increase the strength of the current, or to keep it closed a considerable time, in order to secure making contraction).

4. Intestine. - Place the non-polarizable anode on the intestine of a freshly killed rabbit or frog, the cathode on some indifferent point, for example the liver. Close the key.

The intestine will constrict in the anodal region and remain constricted during the passage of the current, provided it be not so long as to

1 This form was first described in the Catalogue of the Harvarl Apparatus Company, Nay, 1905. 


\section{GENERAL PROPERTIES OF LIVING TISSUES}

cause fatigue. A peristaltic contraction wave usually passes from the anode in both directions along the intestine.

Place the cathode on the intestine, and the anode on an indifferent point. Close the key.

A small, indistinct thickening will be seen in the cathodal region.

Thus the intestine, while it serves admirably to illustrate a polar action of the galvanic current, apparently differs from the tissues already considered in that closure causes contraction at the anode instead of the cathode. The exception is only apparent, and its explanation is that the point at which the electrode touches the peritoneal surface of the many-layered intestinal wall is not the physiological anode or cathode; i. e. not the point at which the current actually enters or leaves the muscular coat. This matter is discussed on page 110 .

5. Smoke a drum. Raise the drum off the friction bearing by turning the screw at the top of the shaft to the right. Arrange two muscle levers ${ }^{1}$ and the electro-magnetic signal (Fig. 27) to write on the drum in the same vertical line. Place the electro-magnetic signal, together with a simple key, in the circuit between one dry cell and the rheochord. Bring the slider near the positive post of the rheochord.

1 Or heart levers (Fig. 53, page 311). 


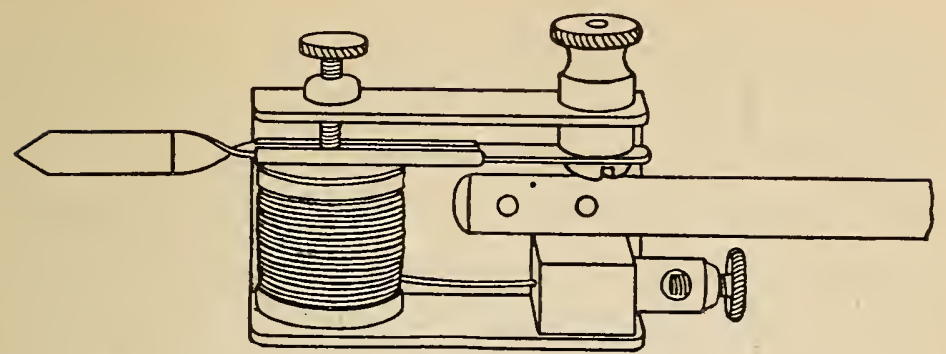

Fig. 27. The signal magnet; the actual size.

The Electro-magnetic signal. ${ }^{1}$ - A protecting metal box (Fig. 27), open at the front and ends, contains a strong magnet, the armature of which is mounted upon a steel spring. An accurate fine adjustment screw resulates the excursion of the armature. One binding post is mounted upon the metal box, the other is insulated by a rubber block. This signal, in circuit with a vibrating tuning fork, will record one hundred double vibrations per second. In the primary circuit of the inductorium it will record the make and break of the current without after-vibrations which are prevented by lead foil placed on the spring where it strikes the limiting screw. Residual magnetism is obviated by parchment paper, fastened to the spring with shellac at the point where the spring would touch the core of the magnet. The handle is long enough to bring the writing point directly above or below the writing point of the muscle lever clamped to the same iron stand.

The metal box is of soft iron and serves as an extension of the magnet core, thus completing the magnetic circuit, and doing away with a second spool. The magnetic power is further improved by boring ont the core, which is then "softened" by heat.

1 First Catalogue of Harvard Apparatus, September, 1901, p. 46. 
Fasten a curarized sartorius muscle by the middle in the Gaskell clamp; the pressure should be enough to prevent the contraction wave of one part reaching the other part, but not great enough to prevent the passage of the excitation. Place the muscle vertical to the writing levers. Tie a thread around the pelvic and tibial fragments and fasten each thread to a muscle lever, so that each half of the muscle may record its contraction independently of the other. Moisten two strands of lamp wick with normal saline clay. Tie one strand around each end of the muscle and lay the free portion of the strands on the toes of boot electrodes properly mounted. Note which lever is connected with the cathodal end. Make the current. If the muscle does not contract, move the slider along the wire a short distance towards the positive post (so as to bring a stronger current through the electrodes) and make the current again. When both make and break contractions are secured, see that the writing points record properly, and "spin" the drum, but not too fast. As soon as the drum moves steadily, make and then break the current.

The moment of making and breaking the current will be recorded by the electro-magnetic signal. An instant later the muscle levers will begin their reeord of the contractions. 
It will be found that the cathodal half of the muscle contracts first on closing, the anodal half on opening the circuit. Evidently the excitation began on closure at the cathode and passed thence to the anode, while on opening the circuit the excitation began at the anode and passed to the cathode.

In order to measure this interval accurately the drum should be turned back until the writing point of the signal lies precisely in the ordinate drawn by it during the experiment. The muscle should then be stimulated. The ordinate now drawn by the muscle with the drum thus at rest will be synchronous with that drawn by the signal during the experiment, and will mark upon the abscissa of the muscle curve the moment of stimulation,

6. Tonic Contraction. - Connect a dry cell through an open simple key with the metre posts of the rheochord. Connect non-polarizable electrodes with the positive post and the slider. Fasten one end of the curarized sartorius (prepared with fragments of pelvis and tibia attached) in the muscle clamp. Tie a thread to the other end and fasten the thread to the upright pin of the muscle lever. Let non-polarizable electrodes rest on the muscle near the respective ends. Use a strength of current that will just cause 
contraction on closure. Watch very closely the cathodal region near the junction of the muscle fibres with the tendon. Close the key.

After the closing contraction, the ends of the muscle fibres next the tendon in the cathodal region will show a faint but distinct thickening, which will remain until the current is broken.

These several experiments demonstrate that in galvanic stimulation of both skeletal and smooth muscle the excitation takes place at the points where the current leaves and enters the muscle. Before inquiring whether this law holds good for the heart, the muscle cells in which have a form intermediate between the smooth muscle cell and the cells of skeletal muscle, it will be necessary to consider whether the points of contact with the electrodes are always the real anode and cathode.

Physiological Anode and Cathode. - When the electrodes are placed directly on a nerve, or are applied to a muscle with straight parallel fibres in such a way that the current flows through each fibre from end to end, the anode and cathode obviously coincide with the points at which the electrodes touch the muscle. When, however, the fibres are of irregular shape, or are irregularly disposed, the current lines can no longer traverse the fibres from end to end, but will enter and 
leave fibres at points other than those in contact with the electrodes.

The difference between the operator's electrodes and the physiological anode and cathode is also obvious when the electrodes are applied to skin, connective tissue, mucous membrane, etc., covering the muscle or nerve, - the points at which the electrodes touch the covering tissue cannot be the points at which the current actually leaves or enters the muscle.

The failure to keep this distinction in mind may lead to wholly erroneous interpretations. Thus when the ureter is extirpated, or is raised from the tissues on which it normally rests, its reaction to the galvanic current follows the law, - contraction begins at cathode on making, at anode on breaking the current; but when the ureter is stimulated in situ, exactly the opposite effect is seen, - contraction begins at anode on making the current. The explanation is that the current lines in the latter case are very widely diffused through the conducting tissues on which the ureter lies, so that the current passes into and out of the muscle fibres for some distance either side of the positive electrode. Each point at which the current leaves a fibre is a secondary cathode, and if the number of such points is large, cathodal stimulation will 
take place in what, superficially regarded, is the anodal region (compare page 131, and Fig. 37). The same explanation holds good for the intestine (see page 103). The formation of physiological anodes and cathodes is well shown in the next experiment.

Physiological Anodes and Cathodes in Rectus Muscle. - Remove the rectus abdominis muscle, from a curarized frog. Note the tendinous cross bands which divide the muscle from side to side and divide it into parts. Lay the muscle smoothly on a glass slide. Connect the nonpolarizable electrodes through a simple key with a dry cell. Place one electrode on each end of the muscle. Close the key.

On closure, the cathodal side of each division of the muscle will show a sharply defined continued contraction of the ends of the fibres at their insertion in the transverse tendinous bands. On opening, the cathodal contraction disappears, and a similar thickening of the fibres is seen at the anodal side of each division. The twitch of each serment on closure and opening of the current also starts respectively from the cathodal and anodal ends of each segment. These effects are best seen through a magnifying glass.

Polar Stimulation in Heart. - The muscle cells of the heart are not only of irregular shape, but 
they are so joined with each other as to make it impossible to pass a current through the heart muscle without the current lines cutting fibres in every direction. It would seem therefore that secondary anodes and cathodes would be formed to such a degree that the demonstration of polar excitation would be difficult or impossible. Experimentation shows however that this is not the case. The heart behaves like a single hollow fibre.

Monopolar Method. - The small size and conical form of the ventricle of the frog's heart make the ordinary method of stimulation, in which the electrodes would both be placed on the heart, less suitable than the monopolar method. This method was suggested by the fact that the stimulating effect of the galvanic current depends on its density. If one electrode has a large surface, and the other a very small surface, the current lines will be distributed through a considerable cross-section in the first instance and converge to a small cone in the second. The threshold value of stimulation will not be reached at the large electrode, and stimulation will occur only at the small electrode. Thus the large "indifferent" electrode may be placed on any part of the frog's body, and the convenient small electrode be used to stimulate the heart. 
Cover the indifferent electrode (consisting of a brass plate furnished with a binding post) with cotton wet with saline solution. Mount a nonpolarizable boot electrode. Con-

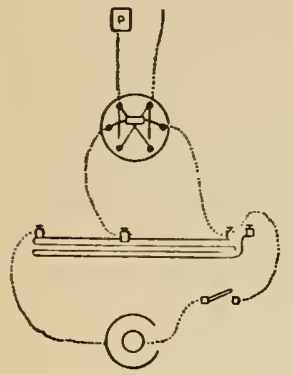

Fig. 2S. nect a dry cell with the metre posts ( 0 and 1 ) of the rheochord through a simple key (Fig. 28). ('onnect post 0 and the slider through a pole-changer (with cross-wires) with the electrodes. Expose the heart, according to the following method: Place the brainless frog, back down, in the holder (Fig. 29). Cut through the skin across the middle of the body from side to side. Make a second cut in the middle line from the

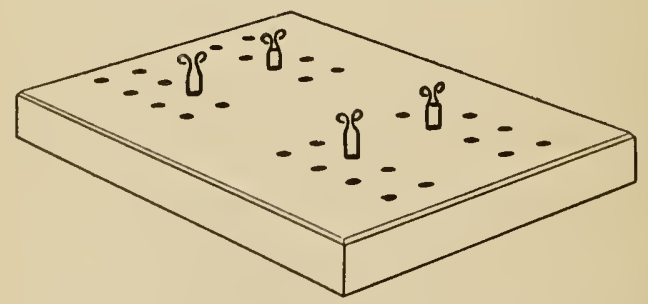

Fig. 29. The frog board, with spring elips ; about one-fourth the actual size.

first cut to near the lower jaw. Turn back the flaps. Cut through the sternal cartilage near its lower end, thus avoiding the epigastric vein. 
Cautiously remove the breast bone, doing no harm to deeper parts. Open the delicate membrane (pericardium) which surrounds the heart. Tie a ligature about the sinus-auricular junction, to stop the ventricular contractions. Place the indifferent electrode over the larynx and the nonpolarizable electrode on the ventricle. Turn the pole-changer so that the electrode on the heart becomes the anode. Close and then open the key.

Contraction will take place on opening only, if at all. Reverse the pole-changer so that the cardiac electrode becomes the cathode. Close and then open the key.

Contraction takes place at closure only.

\section{Polar Stimulation of Nerve}

Law of Contraction. - 1. Whether contraction will follow the galvanic stimulation of a motor nerve depends on the irritability of the nerve and the direction and intensity of the current. The current may pass through the intrapolar portion of the nerve towards the muscle (descending current) or away from it (ascending current). The intensity may be weak, medium, or strong; intensity in this case is evidently merely a relative term, depending on the irrita- 
bility of the particular nerve in hand. We will test first the effect of the ascending current.

Connect a dry cell through an open key with the metre posts of the rheochord (Fig. 30). Join the positive post and the slider through a polechanger (cross-wires in place), with the nonpolarizable electrodes placed in the moist

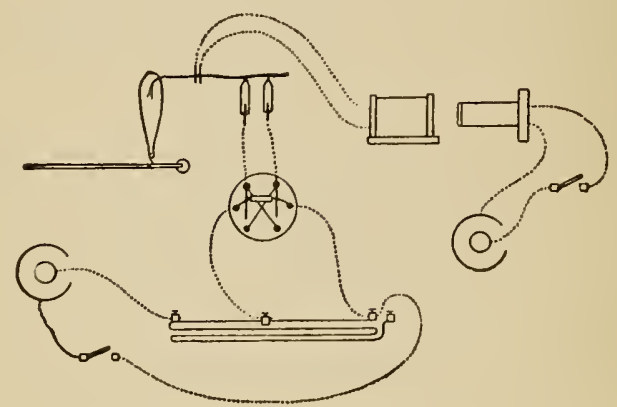

Fig. 30.1

chamber (Fig. 24, page 95), in the holders farthest from the opening for the muscle. Make a nerve-muscle preparation. Secure the femur in the femur clamp of the moist chamber. Let the nerve lie on the non-polarizable electrodes. Attach the Achilles tendon to the muscle lever. Keep the air in the chamber noist by lining the glass shade with filter paper saturated with water. Arrange the pole-changer so that the

1 The inductorium shown in Fig. 30 is not used in this ex- . periment, but in the first experinent on page 116 . 
anode shall be next the muscle. Move the slider near the positive post. Make and break the galvanic current. If no contraction is secured, move the slider to increase the current, and repeat the experiment.

The first contraction will take place on making the current. Continue to increase the current strength by moving the slider.

A point will be reached at which contraction will occur both on opening and closure.

Increase the intensity of the current by adding dry cells in series (zinc to carbon), testing the effect after each addition by closing and opening the current.

An intensity will be reached at which opening and not closure causes contraction.

In a similar manner, work out the law of contraction for descending currents. (It may be necessary to take a fresh nerve-muscle preparation.)

Set down the results in a table.

Intensity of current.

Ascending current. Make. Break.

Contr. Rest. Contr. Rest.

Weak. Medium. Strong.

$$
\text { Contr. }
$$

Contr.

Contr. Contr. Rest. Contr. Contr. Rest (Weak contr.).
Descending current. Make. Break.

2. The remarkable nature of these results is apparent on observing that contraction is easily 
secured on closing a weak ascending current and yet cannot be obtained with a strong one. The first step in the inquiry into the causes of the phenomena is to determine whether the stimulation is polar. That the nerve impulse really starts at the cathode on closure and at the anode on opening is shown (1) by the fact that the interval between stimulation and contraction, with the ascending current, in which the anode is next the muscle, is longer at closure than on opening, while the opposite is the case when the current is descending.

(2) With descending currents, it sometimes happens that opening produces tetanus instead of a simple twitch. If this tetanus appears, the student should sever the nerve between the electrodes. Immediately the contractions will cease. They must therefore have arisen at the anode, for the cathode still remains in full connection with the muscle.

Changes in Irritability. - The second step in this inquiry is to determine the nature of the changes at the poles. For this purpose the nerve should be stimulated in the cathodal and anodal regions during the passage of the constant current.

1. Pass two needles through a cork placed in the rubber holder next the muscle in the moist chamber. Connect them with the secondary coil 
of an inductorium (Fig. 30). Arrange the primary for single induction shocks, which must not be maximal. Turn the pole-changer to bring the cathode next the metal electrodes. Using a weak induction current as stimulus, record on a stationary drum three contractions: (1) before the passing of the galvanic current through the nerve, (2) during its passage, (3) after its passage.

The second contraction - that obtained by stimulating in the cathodal region during the passage of the galvanic current - will be greater than the other two.

Reverse the galvanic current and repeat the experiment, the stimulation now being in the anodal region.

The stimulation in the anodal region during the passage of the galvanic current causes less than the normal contraction.

2. The stimulating current may be superposed directly on the polarizing current by using the same electrodes.

Connect a dry cell through an open key with the 0 and 1 metre posts of the rheochord (Fig. 31). Connect the positive post of the rheochord with one of the non-polarizable electrodes. Join the slider to one end of the secondary wire of an inductorium; to the other end 
join the remaining non-polarizable electrode. If the positive pole of the secondary coil is not known, determine it by the electrolytic method (page 158). Arrange the primary coil of the inductorium for single

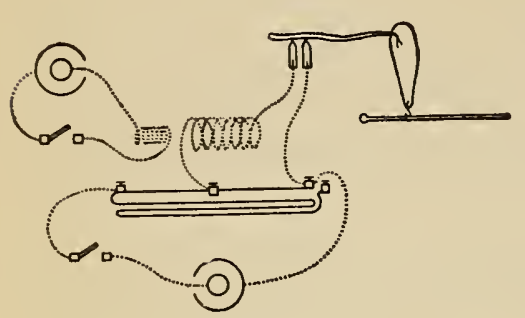

Fig. 31. submaximal induction currents. Make and break the induction current, and record the contractions on the drum. Now pass a weak polarizing current through the nerve and stimulate again with the induction current.

It will be found that the stimulating effect of the induction current is increased when the direction of the induction current coincides with that of the polarizing current, i.e. when the cathode (which is the sole source of the induction stimulus, as pointed ont on page 160) coincides with the cathode of the polarizing current. When the cathode of the induction circuit falls in the anodal region of the polarizing circuit, the stimulating effect is diminished. Very strong polarizing currents produce such alterations in irritability that the additional alteration caused by the bricf induction current is not great enough to be a stimulus. 
The law revealed by this experiment may be thus expressed. The same stimulating current has a greater stimulating effect when it coincides in direction with a pre-existing current, and a lessened effect when it is opposed in direction to a pre-existing current. This law explains the interference observed between stimulating currents and demarcation or injury currents of nerve and muscle (see page 292 ).

3. Place a drop of saturated solution of sodium chloride on the nerve in the extrapolar region near one of the non-polarizable electrodes. Record the irregular tetanus (chemical stimulation) on a slowly moving drum. Make the polarizing current.

Note that the tetanus is increased when the cathode is nearer the stimulating solution, but diminished when the anode is nearer.

Hence the irritability of the nerve is altered during the passage of the electric current (electrotonus) $;^{1}$ it is increased in the neighborhood of the cathode (catelectrotonus) and is diminished in the neighborhood of the anode (anelectro-

1 The change in the excitability of the nerve produced by the electric current is so generally called electrotonus that the term cannot well be changed. It should not be confused with the electrotonus describer on page 000 , though it is possible that the two phenomena have a similar if not identical first cause. 
tonus). In the intrapolar region, the cathodal touches the anodal area at the so-called indifferent point. This point approaches the cathode when the intensity of the polarizing current is increased.

The greater the length of nerve between the electrodes, the greater the extrapolar electrotonus. Catelectrotonus rises rapidly to a maximum as soon as the circuit is closed, and then gradually wanes. Anelectrotonus develops more slowly and does not reach its maximum for some time after closure.

On the opening of the circuit, the conditions at the anode and cathode are reversed, the irritability falls at the cathode and rises at the anode. The fall in the cathodal region is of short duration, and the irritability soon returns again towards normal. In the anodal region, the rise on opening is unbroken.

Changes in Conductivity. - We have seen that the irritability is altered by the galvanic current. The conductivity also is altered.

Connect a dry cell through a pole-changer with cross-wires to a pair of non-polarizable electrodes placed in the holders of the moist chamber farthest from the muscle (Fig. 32). Leave one wire uncoupled until the current is wanterl. Connect another cell with the primary coil of the inductorium arranged for break induction 
shocks, placing in the circuit a simple key and the electro-magnetic signal. Lead wires from the poles of the secondary coil to the side cups of a pole-changer (without cross-wires). In each of the remaining two holders of the moist chamber place a cork pierced by two metal electrodes. One wire in each pair should be insulated from its fellow by rubber tubing drawn over the part between the cork and the end of the electrode to be applied to the nerve. Connect the wire soldered to the basal ends of these electrodes with the remaining cups of the pole-changer in the secondary circuit of the

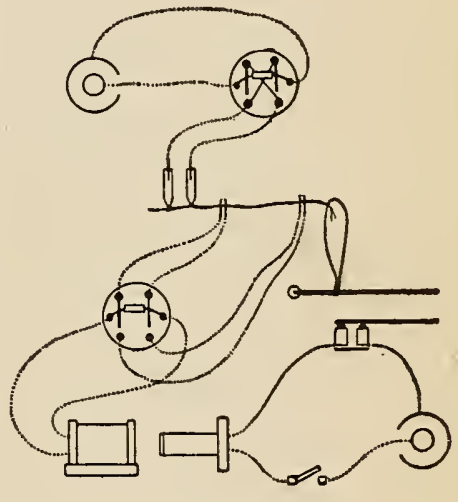

Fig. 32. inductorium. Arrange the signal to write on the smoked drum beneath the writing point of the muscle lever.

Make a nerve-muscle preparation. Let the nerve rest on the non-polarizable electrodes near the cross-section. Place one pair of the metal electrodes beneath the nerve near the muscle, the other pair near the non-polarizable electrodes. The clockwork of the drum should be fully 
wound (not over-wound), and the drum should revolye at its most rapid speed. Write two muscle curves. For the first stimulate through the metal electrodes nearer the muscle; for the second through the metal electrodes farther from the muscle.

While each curve is writing, let a tuning fork record its vibrations beneath the point of the the muscle lever. To mark on the abscissa of the muscle curve the exact moment at which the muscle was stimulated, turn back the drum until the writing point of the signal lies precisely in the line described by it when the current was broken. Now stimulate the muscle with another induction shock. The curved ordinate of the muscle lever will be synchronous with the ordinate of the signal.

The interval between the moment of stimulation, as recorded by the signal, and the beginning of contraction, is greater when the nerve is stimulated far from the muscle. The difference is the time required for the nerve impulse to traverse the length of nerve between the electrodes, provided of course that the interval between the arrival of the nerve impulse in the muscle and the beginning of the contraction is the same in both cases, an assumption considered reasonable by most physiologists. 
Write now three other pairs of curves: one while a galvanic current passes through the nonpolarizable electrodes in a descending direction (cathode nearer the muscle); a second while an. ascending current passes (anode nearer the muscle); and a third, after the galvanic current has been some minutes broken, as a control. During the writing of these curves measure the velocity of the drum with the tuning fork as before.

The speed of the nerve impulse will be found to be greater than normal when the nerve impulse starting at the second pair of metal electrodes passes through an extrapolar cathodal area (i.e. stimulation during descending current), and less than normal when that region is made anodal by reversing the galvanic current. In other words, the conductivity of the nerve has been increased by cathodal and diminished by anodal stimulation.

2. Conductivity is diminished by strong or protracted currents in the cathodal as well as in the anodal region. - Place two non-polarizable electrodes upon the nerve about $3 \mathrm{~cm}$. apart. Connect them through a pole-changer with two dry cells (Fig. 33). In the middle of the intrapolar region place two stimulating electrodes close together. Connect one of the stimulating electrodes directly to the secondary coil of an induc- 


\section{GENERAL PROPERTIES OF LIVING TISSUES}

torium arranged for single induction currents. Lead from the other stimulating electrode to a piece of nerve or muscle about $4 \mathrm{~cm}$. long, and thence to the secondary coil. The introduction of this great resistance will keep most of the polarizing current in the short bridge of nerve between the polarizing electrodes. Without this resistance, the polarizing current would pass

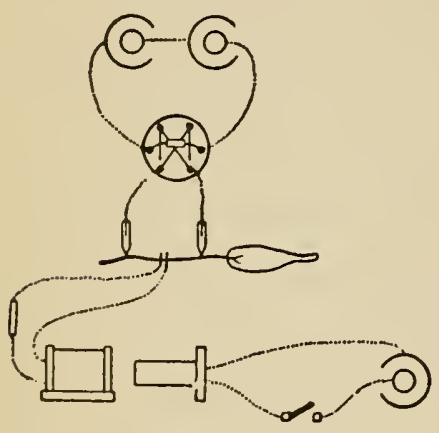

Fig. 33.

through the stimulating circuit in preference to crossing the nerve between the stimulating electrodes. Observe that the nerve impulse created by the stimulus must pass through the cathodal region, if the current be descending, or the anodal region, if the current be ascending, in order to reach the muscle.

Find the position of the secondary coil at which the muscle will barely contract on making the stimulating current. Arrange the polechanger to bring the anode between the stimulating electrodes and the muscle, and make the polarizing current. Stimulate with a make induction current during the passage of the polarizing current. Open tihe polarizing current. 
After three minutes' rest, bring the cathode next the muscle and make the polarizing current as before. Then stimulate again with a make induction current of the same intensity as before.

Contraction will be absent, or at most very weak. The impulse will be blocked in the cathodal region. In truth, during the passage of strong or protracted currents, the conductivity is more diminished in the cathodal than in the anodal region.

Griitzner and Tigerstedt believe that the opening contraction is due to the stimulation of the nerve or muscle by the polarization current which appears when the galvanic current is broken. The polarization current may be said to be closed when the galvanic current is opened. These observers, therefore, hold that stimulation takes place only at closure.

We are now in a position to account for the phenomena described by the law of contraction. The irritability of the nerve is increased at the cathode on closing, and at the anode on opening the galvanic current. This rise of irritability stimulates the nerve. The rise at the cathode is a more effective stimulus than the rise at the anode; consequently with weak currents the first stimulus to produce contraction is cathodal, $i$. $e$. at the closure of the circuit. As the current in- 
tensity is increased, the anodal rise becomes also effective, and contraction is secured by both making and breaking the current.

But we have to deal also with a decrease in irritability, and, still more important for the explanation of the effects of strong currents, with a decrease in conductivity. The irritability and conductivity are decreased on closure at the anode and on opening at the cathode. If the anode is next the muscle (Fig. 34), the decrease in conductivity on closure of a strong current will block the nerve im$\longrightarrow$ i_ pulse coming from the cathode; it will therefore never reach the

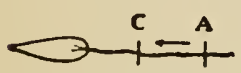

Fig. 34. muscle, and there will be no contraction on closure. If the cathode is next the muscle, the conductivity may be so decreased on opening that the nerve impulse coming from the anode may be blocked. The decrease at cathode, when the current is broken, is, however, less marked than the decrease at anode when the current is made, so that the cathodal decrease, even with strong currents, sometimes fails to block the impulse entirely. In that case, a weak contraction may be obtained at the break of the descending current. 


\section{Stimulation of Human Nerves}

Duchenne devised a method by which either the motor or the sensory human nerves can be stimulated at will, and the reaction of single muscles or groups of muscles to electricity determined. When electrodes are placed on the surface of the skin and the circuit is made, the current entering at the anode will spread in current lines through the entire body. At the cathode, all these lines will converge again. The density of the current depends on the concentration of the current lines. Thus the density is relatively great at the electrodes, and becomes rapidly weaker as the lines diverge between them. The smaller the electrode, the greater the density. The stimulating effect depends on the density. With small electrodes, a current not sufficient to cause stimulation may gradually be increased in strength until the density at the electrode becomes great enough to stimulate, while in all other regions it is not yet great enough. Thus a local stimulation is secured. But this local stimulus does not sufficiently distinguish between the sensory nerves and the motor nerves and muscles; for in order to reach the deeper lying motor nerves and muscles, the current must pass through the skin. The resistance of the epidermis is very great, and 
currents of considerable intensity are necessary to overcome it. Once through the epidermis, the current spreads immediately in all directions through the cutis, where it stimulates the very

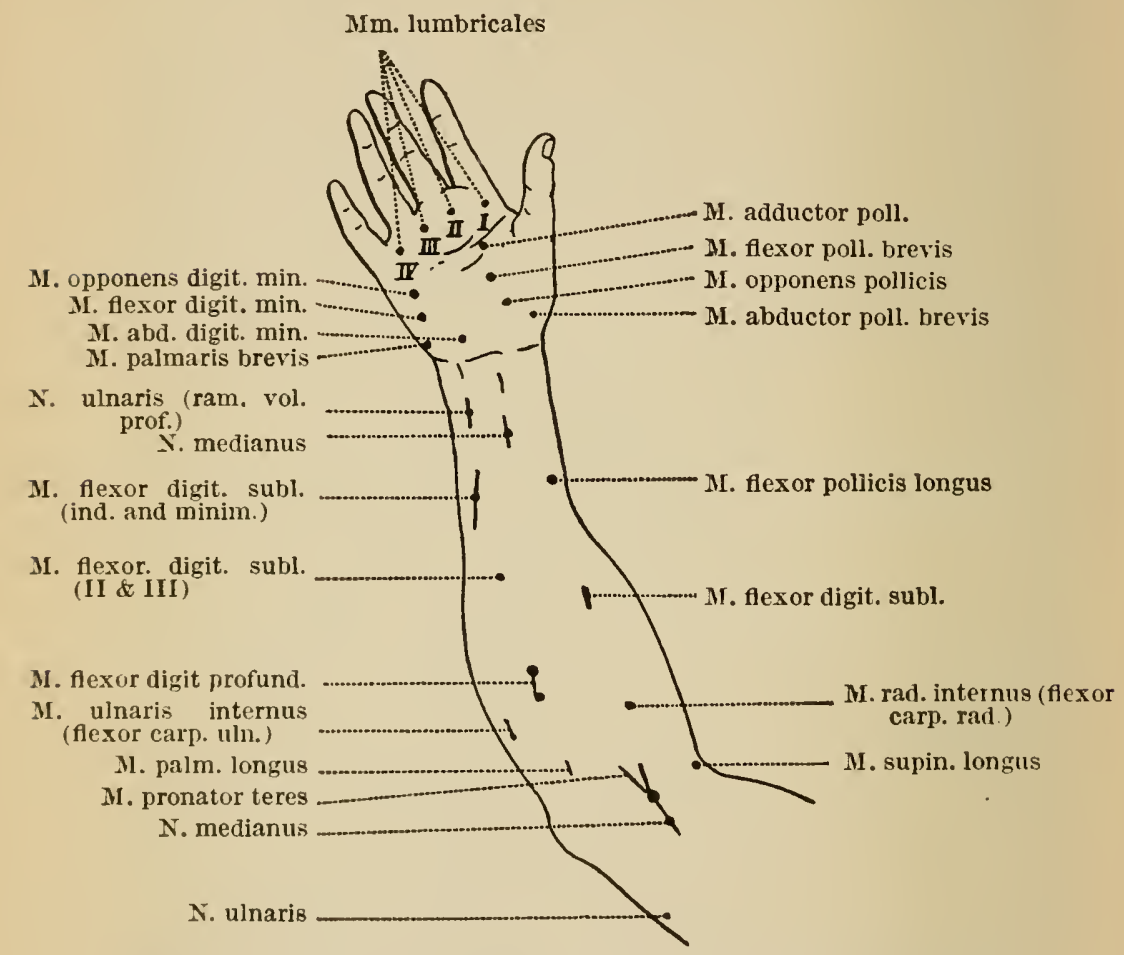

Fig 35. The motor points on the anterior surfuce of the forearm and hand.

numerous sensory nerves. When the muscles or motor nerves are reached, the density is much reduced, and may not suffice for stimulation. Thus the result may be not motor stimulation, but simply pain from stimulation of the sensory 
nerves. For painless motor stimulation it is, therefore, necessary to increase the strength of the current which reaches the muscle or motor nerve and to diminish the density of the current

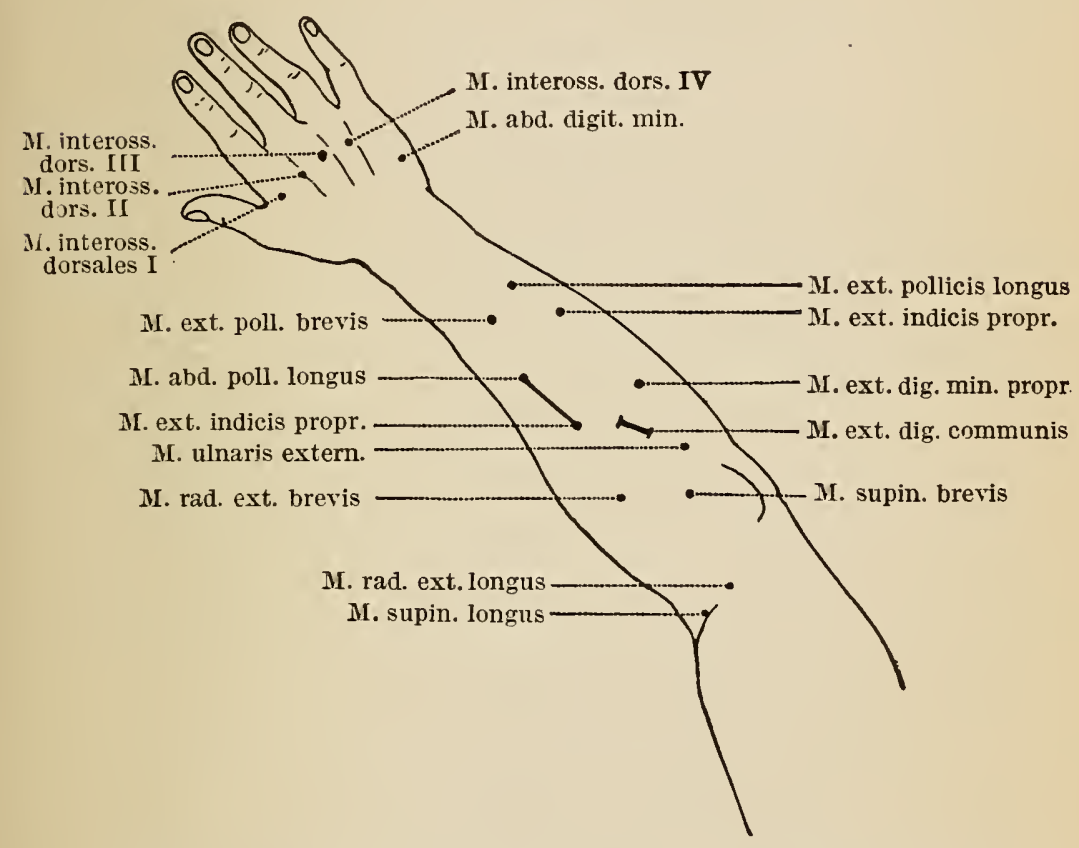

Fig. 36. The motor points on the posterior surface of the forearm and hand.

at the electrodes. These ends are accomplished by using for electrodes large metal plates covered with sponge or cotton wet with saline solution. The liquid diminishes greatly the resistance of the epidermis, so that more current reaches 
the deeper tissues; and the large surface offers a broad path for the current, so that the current lines are not so concentrated as to stimulate painfully the sensory nerves of the cutis. One sponge electrode may be made considerably smaller than the other without forfeiting this advantage, while the smaller size makes it easier to localize the stinuulus.

Muscles are best stimulated through their nerves, for two reasons: the nerve responds to a weaker stimulus than the muscle; and, secondly, it is much easier to secure contraction of the whole muscle by stimulating the nerve than by attempting to pass a current through the muscle directly. The smaller electrode should be placed over the nerve, the larger on some indifferent region. The indifferent electrode may be placed over the muscle itself, if it is important that the resistance shall not be increased by the too great separation of the electrodes.

Juchenne found that certain points were especially favorable for the stimulation of individual muscles. Remak discovered that these "motor points" were simply the places at which the nerves entered the muscle. The motor points of the forearm are shown in Figs. 35 and 36.

Stimulation of Motor Points. - Arrange the inductorium for single induction shocks. Determine by the electrolytic method which pole 
of the secondary coil is the cathode when the primary current is broken (page 158). To this pole connect the small (stimulating) electrode; to the other pole connect the large (indifferent) electrode. Place the indifferent electrode on the arm or neck. With the small electrode make out the motor points indicated in Figs. 35 and 36 .

Polar Stimulation of Human Nerves. - In the hands of the earlier observers the stimulation of nerves within the body gave results often contrary to the law of polar stimulation so easily demonstrated in extirpated nerves. The explanation of these inconstant results lay in the failure to comprehend the distinction between the stimulating positive and negative electrodes and the physiological anode and cathode (compare page 108). Even when the monopolar method is employed, and a small electrode is brought as near as possible to the nerve to be stimulated, while a large indifferent electrode is placed on some other part of the body, it is impossible to secure true monopolar stimulation. The current entering at the anode does not remain in the nerve, but very soon passes out into the surrounding tissues (Fig. 37). Hence there are physiological cathodes on both sides of the positive electrode, and for the like reason physiologi- 
cal anodes on both sides of the negative electrode. Thus both anodal and cathodal stimulation take place, whichever electrode rests over the nerve. It is therefore incorrect to speak of ascending and descending currents in the case of nerves stimulated in situ. It should be

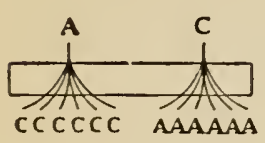

Fig. 37.

pointed out, too, that the density of the current is greater on the side of the nerve nearer the electrode than on the more deeply placed side cut by current lines already rapidly diverging.

With these facts in mind, we may compare the polar stimulation of human nerve with the law already determined for the isolated nerves of the frog (page 115).

The Brass Electrodes. - The brass electrodes, used chiefly for the stimulation of human muscles

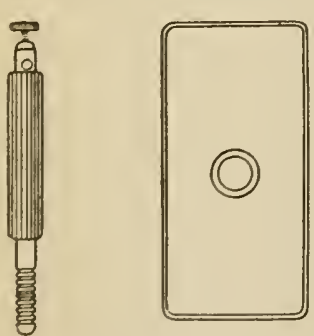

Fig. 38 . and nerves, are two in number: an "indifferent" electrode, consisting of a brass plate, $3 \times 6$ cm., with binding post, and a "stimulating" electrode, of brass rod, $6 \mathrm{~cm}$. long, ringed at one end and provided at the other with a binding post. Between these the rod is insulated with rubber tubing.

The electrodes should be covered with cotton wet 
with normal saline solution. The larger electrode may be fastened upon the arm or other indifferent region, and the smaller may be used to stimulate the nerves or muscles, for example the abductor indicis, or to find the "motor points."

Connect 8 dry cells in series (the carbon of one cell to the zinc of the next, etc.). Coupling in this way enables the electromotive force of each cell to be added with slight loss to that of the others, provided the resistance in the circuit outside the cells is so great that the internal resistance of the battery disappears in comparison, as is the case where living tissues form part of the circuit. Connect the terminal zinc and carbon pole through a pole-changer (with crosswires) to a small and a large electrode covered with cotton thoroughly wet with strong saline solution. Place the small electrode over the ulnar nerve between the internal condyle and the olecranon, a little above the furrow. Make and break the current. If no contraction is secured, add cells to the battery until contraction occurs.

It will be found that the first contraction occurs on closure with the cathode over the nerve. With this strength of current the opening contraction will be absent.

Turn the pole-changer so as to bring the anode 
over the nerve, and increase the intensity still further.

A strength will be reached at which closure with the anode over the nerve will cause contraction, but the opening of the current will still be without effect. A slightly greater intensity will now bring out the anodal opening contraction. ${ }^{1}$

In the mean time the cathodal closing contraction has increased in force with each addition to the intensity of the current. With about 18 cells, the muscle twitch on closure may give place to a continued contraction or tetanus, the cathodal closing tetanus. Further increase gives cathodal opening contraction, and finally very strong currents sometimes cause anodal closing tetanus. Thus we have

1. Cathodal closing contraction.

2. Anodal closing contraction.

3. Anodal opening contraction.

4. Cathodal closing tetanus.

5. Cathodal opening contraction.

6. Anodal closing tetanus (rare).

Sometimes the anodal opening precedes the anodal closing contraction.

1 Sometimes anodal opening contraction precedes the elnsing contraction. This inconstancy results from variations in current strength due to differences in the tissues surrounding the nerve. 
The apparent deviation from the law of polar excitation (cathodal on closure, anodal on opening) is explained by the presence of a physiological anode and cathode at each electrode, as already mentioned. The appearance of cathodal closing contraction before anodal closing contraction is due to the fact that when the negative electrode lies over the nerve the physiological cathode will be found on the side of the nerve next the electrode. The nearer the electrode, the greater the current density, and hence the earlier the threshold value is reached. When, however, the positive electrode lies over the nerve, the physiological cathode will be found on the side of the nerve farther from the electrode, where the density is less, owing to the divergence of the current lines. The threshold value will be reached first at the point of higher density, and consequently the first contraction will appear while the negative electrode rests over the nerve. The anodal opening contraction appears before the cathodal opening contraction for a similar reason.

Reaction of Degeneration. - Whenever a nerve is severed, the portion separated from the cell of origin of the nerve "degenerates." The degeneration does not begin at the section and advance to the terminal branches, but takes place al- 
most or quite simultaneously throughout the nerve. Ranvier states that it begins first in the end plates. Severed nerves in the brain and spinal cord degenerate in the same way, and this "Wallerian degeneration" (Waller, 1850) is a valuable aid in tracing the path of nerve fibres in the central nervous system. Degeneration is accompanied by changes in the reaction to the electric current which form a valuable aid in the diagnosis of the seat of the lesion in cases of paralysis. The muscle reacts imperfectly, or not at all, to the brief induction current, while its reaction to the long galvanic current may even be greater than usual.

Expose each gastrocnemius muscle in a frog, the left sciatic nerve of which has been severed ten days before this experiment. Stimulate each muscle with weak induction currents and with the galvanic current.

The muscle, the nerves of which are degenerated, reacts more readily to the galvanic current than to the brief induction current. The normal muscle shows the opposite reaction.

In man, the reaction of degeneration in the case of muscle consists of a lessened or lost excitability to the induced current with increased excitability to the galvanic current. The duration of contraction may be greater than normal. In 
polar stimulation, anodal closing contraction may appear before cathodal closing contraction, - a reversal of the normal sequence.

In degenerated nerve there is of course a total loss of irritability, corresponding to the destruction of the axis-cylinder.

\section{Galvanotropism}

Paramecium. - Connect two non-polarizable electrodes through a pole-changer with a dry cell. On a glass microscope-slide make with wax an enclosure about one centimetre square and a few millimetres high. Place in this a little hay infusion containing Paramecia. Bring near the two opposite sides of the wax cell non-polarizable electrodes, provided with a thick thread that shall dip into the infusion. Examine the infusion with a very low power. Close the key.

Upon closure each Paramecium turns the anterior end of the body towards the cathode and swims in that direction. In a very short time the anodal region is free, and the Paramecia are gathered at the cathode, where they remain so long as the current flows.

Change the direction of the current.

The Paramecia now turn to the anode and swim in that direction, but the anodal grouping is less complete than the cathodal, and lasts but 
a short time. Careful observation shows that in Paramecium the galvanic reaction consists in placing the long axis of the body in the current lines. The outermost individuals in the liquid will therefore describe a curve corresponding to the curved outer current lines.

All protozoa and many other animals (for example, the tadpole and the crayfish) show galvanotropism, but in some, movement on closure is toward the positive pole (positive galvanotropism).

These experiments on skeletal, smooth, and cardiac muscle, on nerve, and on infusoria, suggest that polar excitation occurs wherever a galvanic current passes through irritable tissue. Further experience would confirm this view. We have seen that the changes at the eathode when the current is made are not momentary, as required by the hypothesis of DuBois-Reymond, but continue so long as the current flows. This fact appears still more clearly when the influence of the duration of the current is examined.

\section{Influence of Duration of Stimulus}

1. Smoke a drum. Arrange a muscle lever to write on the smoked paper. Prepare non-polarizable electrodes and fasten them on the glass plate 
of the nerve holder. Arrange the inductorium for maximal induction currents. Lead from the secondary coil to a pair of the end cups of the pole-changer (without cross-wires), as in Fig. 39. To the opposite cups of the pole-changer bring wires from a dry cell. Connect the remaining cups with the non-polarizable electrodes. Turn the rocker towards the induction coil. Fasten the pelvic attachment of the curarized sartorius in

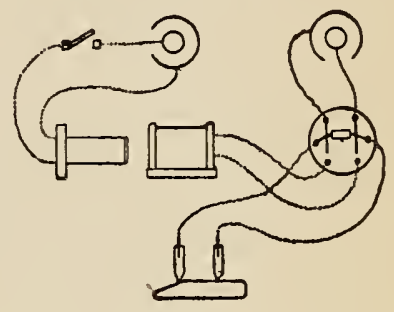

Fig. 39. the muscle clamp. Tie a thread to the fragment of tibia, and fasten the thread to the upright pin of the muscle lever, so that the horizontal muscle shall record its contraction on the drum. 'Start the drum at moderate speed. Record contractions, (1) with maximal break shocks, (2) with closure of galvanic current. Compare the curves.

The curve from galvanic stimulation will be of greater height and duration, and the summit of the curve will be less pointed, indicating that the muscle remains longer in the stage of extreme shortening.

Other evidence that the duration of the stimulus modifies the character of the contraction is afforded by the following experiments :- 
2. Make two cuts, 5 $\mathrm{mm}$. apart, through the frog's stomach at right angles to the long axis. Hang the ring thus secured in the moist chamber. Pass a bent hook through the lower end of the ring, and attach it by means of a fine copper wire to the hook on the muscle lever. Carry the end of the copper wire to the binding post on the muscle lever.

Stimulate not more than twice with single induction currents of a strength about the threshold value for skeletal muscle of frog.

There will be no contraction.

Stimulate with galvanic current (two dry cells), writing three curves, the duration of closure being approximately one-fifth second, one, and five seconds, respectively. Compare the curves.

The maximum shortening with currents of brief duration ( $\frac{1}{5}$ second) is very much less than with currents of three or four seconds or over. The briefer the current also, the quicker will the maximum shortening be reached, and the quicker will be the relaxation.

3. If the galvanic current is very rapidly made and broken, the muscle will not contract.

1 If the muscle does not respond, wrap it with filter paper moistened with nornal saline solution, and wait until the tonic contraction due to the cutting has passed off. The tonus may sometimes be lessened by passing a galvanic current through the preparation (p. 153). 
The same is true of the ureter (Engelmann).

4. Tonic Contraction. - Examine the contraction curve already recorded by the smooth muscle of the frog's stomach. Note that the muscle remains contracted during the passage of the current. The curves secured from the curarized sartorius (page 139) also show this, but to a much less degree; the sartorius does not resume its former length after the twitch or closure of the galvanic current, but remains contracted to a slight extent. This tonic contraction appears much more plainly in fatigued muscles.

Fatigue a sartorius muscle by stimulating it with a galvanic current repeatedly made and broken. After a time, the twitch on closure will become very feeble, and finally will disappear, while the tonic shortening during the passage of the current is still very evident.

5. The influence of duration is shown also in the opening contraction.

Fasten the pelvic attachment of a sartorius muscle in the muscle clamp and connect the other end with the upright pin of the muscle lever, so that the horizontal muscle shall record its contraction on a drum. Place the non-polarizable electrodes on the ends of the muscle. Allow the galvanic current from a dry cell to 
pass through the muscle until the closure tonic contraction has disappeared, then open the key. Neglect the opening twitch.

The muscle will not return to its original length, but will remain contracted for a time (opening tonic contraction).

Close the key again.

The tonic contraction will disappear.

The galvanic current in this case checks (inhibits) a contraction. This new action is discussed on page 153.

6. Rhythmic Contraction. - That the galvanic current acts as a stimulus so long as it continues to flow is shown also by the fact that its passage through contractile tissue may cause the muscle to fall into rhythmic contractions. These are easy to produce in muscles which normally contract in rhythms, for example, the heart; but they may under some circumstances be observed also in smooth muscle, and even in skeletal muscles.

Connect a dry cell through a simple key with the metre posts of the rheochord. Join the nonpolarizable electrodes to the positive post and the slider. Bring the slider against the positive post, so that no current shall flow through the electrodes when they are joined by the tissue.

Expose the heart. With a sharp knife bisect 
the ventricle transversely. Rest this "apex" preparation between the tips of two non-polarizable boot electrodes. Keep the tissue moistened with normal saline solution, but avoid excess. Close the key. Move the slider along the wire.

When the current taken off reaches the threshold value, the apex will begin to beat rhythmically. Increasing the current strength will increase (within limits) the frequency of contraction.

Skeletal Muscle. - The curarized sartorius may sometimes be brought into rhythmic contraction by constant currents (Hering). If the irritability of the muscle at the point of stimulation be increased by applying to the cathodal region a two per cent solution of sodium carbonate, the constant current will produce strong rhythmic contractions.

Smoke a drum. Fasten the pelvic end of the sartorius in the muscle clamp, and attach the tibial end by a thread to the vertical pin on the muscle lever so that the horizontally extended muscle may write its contraction on a drum. Lay on the tibial fifth of the muscle a piece of filter paper, wet with two per cent solution of sodium carbonate. Connect a dry cell through a simple key with the metre posts of the rheochord. Connect the non-polarizable electrodes 
with the positive post and the slider. Bring the slider near the positive post. When the sodium carbonate has acted for 15 minutes, bring the cathode against the tibial end, the anode against the pelvic end of the muscle. Close and open the circuit, moving the slider meanwhile to find the current which will give closing contraction. At this point keep the circuit closed.

Rhythmical contractions usually appear.

Periodic contractions are observed also in smooth muscle, stimulated with the constant current. Any form of constant stimulus will serve to produce them, pressure - as in the heart, bladder, and intestine - and chemical action, being especially noteworthy.

Continuous Galvanic Stimulation of Nerve may cause the Periodic Discharge of Nerve Impulses. If two non-polarizable electrodes are allowed to rest on the inuscle (horizontally suspended), and are connected to a capillary electrometer, the meniscus of which is projected through a slit onto rapidly moving sensitized paper, the shadow of the meniscus will make a straight line on the photographic paper so long as the muscle is at rest. When, however, the nerve of the muscle is stimulated with the galvanic current and closing tetanus appears, the straight line will be broken by $10-15$ oscillations per second. These 
oscillations are produced by the difference of potential created by each contraction wave as it passes over the muscle (contracting muscle is negative towards muscle at rest, see page 302), and demonstrate that the tetanus is a fusion of individual contractions produced by successive stimuli.

Hence, nerve, like muscle, responds to a continuous stimulus by a periodic discharge of energy.

Ulnar Nerve. - Connect 15 dry cells in series (zinc to carbon), and join the last zinc and carbon through a key to a small brass stimulating electrode one $\mathrm{cm}$. in diameter, and a large "indifferent" electrode (brass plate $6.5 \times 3.5 \mathrm{~cm}$. covered with cotton wet in solution of common salt). Hold the indifferent electrode in the left hand, and apply the stimulating electrode to the ulnar nerve at the elbow.

A peculiar tingling sensation will be felt so long as the current flows.

Polarization Current. - Let the sciatic nerve rest on a pair of non-polarizable electrodes in the moist chamber. Connect the electrodes to the side cups of the pole-changer (without crosswires). Connect one end pair of the pole-changer cups with a dry cell. Turn the rocker to the opposite side to prevent the battery current from reaching the electrodes until it is wanted. Con- 
nect the remaining pair of cups through a closed short-circuiting key with the capillary electrometer. Let the galvanic current flow some minutes through the nerve, then turn the rocker towards the electrometer and open the shortcircuiting key.

Note a movement of the meniscus in a direction indicating that the former cathode is now positive to the former anode.

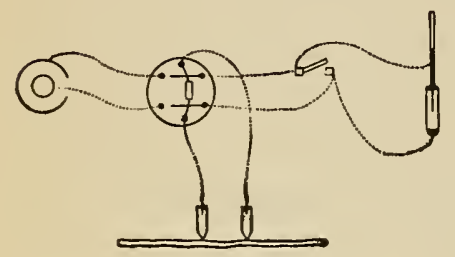

Fig. 40. The nerve is polarized. Positive Variation.If the polarizing current is strong and brief, the negative polarization after-current will speedily give place to a positive current, i.e. one in the direction of the polarizing eurrent. This positive current is really an action current. When the polarizing current is broken, the rise of irritability at the anode stimulates points nearer the anode more strongly than points farther away. Points nearer the anode become, therefore, negative to points farther away, and a current flows through the electrometer circuit from the less negative to the more negative pole, and through the nerve in the direction from anode to cathode. This positive variation is seen only in living nerves. 
Polar Fatigue. - Connect non-polarizable electrodes through a simple key with a dry cell. Fatigue a sartorious muscle by opening and closing the galvanic circuit (leave a brief interval between opening and closure). Closure will at length be followed by no contraction. Arrange an inductorium for single induction currents (the pole-changer may be placed in the primary circuit as a simple key). Test now the irritability of the muscle by stimulating it with single induction currents.

The muscle will be irritable except in the cathodal region. The fatigue has been local (polar).

Opening and Closing Tetanus. - 1. Arrange a moist chamber with a muscle lever to write on a smoked drum. Place two non-polarizable electrodes in the moist chamber and connect them through a pole-changer with a dry cell. Make a nerve muscle preparation from a frog that has just been brought from a cold room into the warm laboratory. Secure the femur in the femur clamp of the moist chamber. Let the nerve rest on the non-polarizable electrodes. Attach the muscle to the lever. Bring the writing point against the slowly moving drum. Close the key.

If the frog has been well cooled (below $10^{\circ} \mathrm{C}$.), the muscle will fall into tetanus both on closing and on opening the circuit. Note that the curve 
is quite regular. If tetanus fails to appear, paint the cathodal region with one per cent solution of sodic carbonate, thus raising the irritability, and repeat the experiment. The curve secured in this way is likely to be irregular.

Produce opening tetanus, and while the muscle is contracting close the current again.

The tetanus will disappear; the irritability will be reduced in the anodal region, from the polarization of which the tetanus was produced.

Open the current again. When the tetanus reappears reverse the pole-changer and close the current.

The tetanus will be increased; the irritability in the former anodal region will suffer a catelectrotonic increase.

2. A beautiful demonstration of polar excitation may be made in this experiment. Connect the electrodes in such a way that the intrapolar current shall be descending (i.e. towards the muscle). When the opening tetanus appears, cut away the anode by severing the nerve between the electrodes.

The contraction ceases with the removal of the source of stimulation.

3. The stimulating effect of the salts of the alkalies has been explained by their attraction for water, the loss of which increases the effect 
of the galvanic current on nerve. When the irritability of the nerve is raised by drying, weak currents may give opening contractions, although they are absent in normal, uninjured nerves. The interval between the opening of the current and the resulting contraction is then markedly long. In nerves in the first stage of drying the intensity of the nerve impulse (height of contraction of attached muscle) is also more than usually dependent on the duration of the current.

4. The opening tetanus (so-called Ritter's tetanus) is probably caused by the rise of irritability, which takes place in the anodal region when the current is shut off, acting on a nerve already in latent excitation. A similar condition can be produced as follows :-

Smoke a drum. Connect a dry cell through an open key and an electro-magnetic signal with the metre posts of the rheochord (Fig. 41). Connect the zero post and the slider of the rheochord with the pole-changer (with cross-wires), and the latter with two non-polarizable electrodes placed in the moist chamber. Make a nerve-muscle preparation, and secure the femur in the femur clamp of the moist chamber. Attach the muscle to the muscle lever. Bring the writing points of the muscle lever and the electro-magnetic signal 
against the smoked surface in the same vertical line. Let the nerve rest on the non-polarizable electrodes. In the remaining two posts in the moist chamber fasten stimulating electrodes. Connect the latter to the inductorium, arranged for tetanizing currents, short-circuiting key closed. Bring the stimulating electrodes against the nerve between the non-polarizable electrodes and the

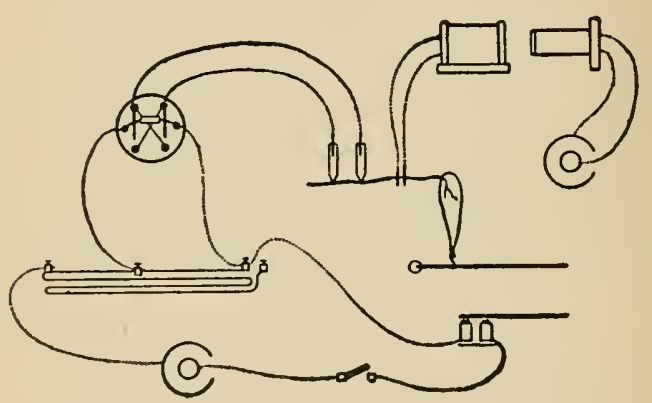

Fig. 41.

muscle. Let the secondary coil be at such a distance that the tetanizing current will be just below the threshold value. Turn the pole-changer so that the anode shall be next the tetanizing electrodes. Make and break the galvanic current, recording the contraction on a slowly moving drum. Now open the short-circuiting key, and after half a minute, and while the sub-minimal tetanizing current is still passing through the 
nerve, make and break the galvanic current again.

A moderately strong galvanic current will now produce an opening tetanus (anodal stimulation of a region the irritability of which has been raised by the sub-minimal tetanizing current). Other effects are a lengthening of the latent period, and an increased dependence on the duration of the galvanic current (see page 138).

Reverse the pole-changer, so that the tetanizing electrodes fall in the cathodal region. Repeat the experiment, comparing the results of cathodal stimulation without and with the sub-minimal tetanizing current.

With sub-minimal tetanization, an increase in the height of the closing contraction, when the galvanic current is not too strong, will be seen; when the galvanic current is stronger, closing tetanus will also be observed.

Polar Excitation in Injured Muscle. - Smoke a drum. Make non-polarizable electrodes. Connect a dry cell through a simple key and pole-changer (with cross-wires) with the nonpolarizable electrodes. Prepare a sartorius muscle with bony attachments. Fasten the pelvic end in the muscle clamp. Tie a thread to the tibial end, and fasten the thread to the upright pin of the muscle lever, so that the muscle is extended 
horizontally. Bring the writing point against the drum. Light a Bunsen burner. Heat a wire, and kill the pelvic end of the muscle by laying the hot wire against it. Bring one non-polarizable electrode upon each end of the muscle. Arrange the pole-changer so that the cathode shall be at the pelvic end, and the current therefore "atterminal," $i$. e. directed toward the "thermal cross-section." Close the simple key.

No contraction, or a very slight contraction, will be seen.

Open the key. Reverse the pole-changer, so that the current shall be "abterminal." Close the simple key.

The ordinary closing contraction will be seen.

The great difference here shown between the polar excitability in the uninjured and injured region is probably due to chemical changes in the injured part. Similar results can be obtained by painting the end of the muscle with one per cent solution of acid potassium phosphate. The irritability is lessened by this salt, but returns to normal if the altered end of the muscle is bathed in 0.6 per cent sodium chloride solution.

Sodium carbonate has an effect opposite to that of the potassium salts.

Wet the pelvic end of a fresh muscle with one per cent solution of sodic carbonate. After a 
short time, test the irritability to weak, ascending (i.e. cathode at pelvic end) currents.

The closure of ascending currents will give extraordinarily large contractions.

The cause of this change in irritability is not the presence of dead contractile tissue, for electrodes can be wrapped in dead muscle and used to stimulate normal muscle without loss of irritability being noticeable.

When the end of the fibre is killed, the pathological change passes gradually through the whole of the fibre.

\section{Polar Inhibition by the Galvanic Current}

It remains now to consider the inhibitory action of the galvanic current, to which attention was called on page 142 .

Heart. - Connect a dry cell through a simple key with the 0 and 1 metre posts of the rheochord. Connect non-polarizable electrodes through a polechanger with cross-wires (Fig. 30), with the slider and the positive post of the rherchord. Pith the brain, not the cord, of a frog, and place the animal, back down, in the holder (Fig. 29, page 112), and expose the heart, without unnecessary loss of blood, according to the method described on page 112. Open the delicate membrane (pericardium) 
which surrounds the heart. Let one electrode rest on the larynx. Lay upon the tip of the other electrode a strand of lamp wick or absorbent cotton wet with normal saline solution. Bring this electrode over the heart so that the free end of the strand rests on the ventricle and moves with it. Turn the pole-changer to make this electrode the anode. Make the current.

At each systole, the portion of the ventricle immediately about the anode will not contract with the rest, but will remain relaxed (local diastole). Thus while the greater part of the ventricle becomes pale as the blood is squeezed out of its wall by the contraction, the anodal region remains dark red. From this region the relaxation spreads over the rest of the ventricle. Reverse the pole-changer. Break the current.

The cardiac electrode is now the cathode. In the systole following the breaking of the current, the cathodal region will remain relaxed during contraction of the ventricle.

This experiment demonstrates that the galvanic current not only may stimulate, but may check or inhibit contraction. In the former case, the conversion of potential into active energy is set going; in the latter, it is prevented. Inhibition plays a large part in the physiology of the day. 
Polar Inhibition in Veratrinized Muscle. - A similar inhibitory effect can be demonstrated in skeletal muscle previously placed in continued ("tonic") contraction by veratrine poisoning. Inject with a fine glass pipette seven drops of one per cent solution of veratrine acetate in the dorsal lymph sac of a frog.

Arrange two muscle levers to write on a drum. Between them place an electromagnetic signal. Let all three writing points be in the same vertical line. Connect a dry cell through a simple key with an inductorium arranged for single induction snocks. Connect non-polarizable electrodes through another simple key and the electromagnetic signal with a dry cell. Prepare a sartorius muscle with pelvic and tibial attachments. Fasten the muscle about the middle in the cork clamp. Fasten the cork clamp vertically in the jaws of the muscle clamp. Carry threads from each end of the muscle to one of the muscle levers. Place the non-polarizable electrodes near the respective ends of the muscle. Note which is the anode. Bring wires from the secondary coil of the inductorium to the ends of the muscle. Start the drum moving slowly. Stimulate the muscle with a single induction shock. There will be a prolonged contraction, characteristic of veratrine poisoning. So 
soon as this contraction is well under way, make the constant current.

The anodal half of the muscle will show a distinct relaxation; the cathodal half will not relax, but may even contract a little more.

\section{Stimulation affected by the Form of the}

\section{Muscle}

Connect a dry cell through a simple key to the metre posts of the rheochord. Bring wires from the non-polarizable electrodes to the positive post and the slider, interposing the pole-changer with cross-wires so that the direction of the current can be changed. Place the slider against the positive post, so that all the current passes back to the cell.

Prepare a curarized sartorius muscle with its bony attachments. Fasten the pelvic fragment in the muscle clamp. Tie a thread about the tibia and fasten the thread to the upright pin of the muscle lever. Let the cathode rest on the tibial end of the muscle, the anode on the pelvic end; the current will then be descending. Move the slider a few centimetres away from the positive post, and make the current. If no contraction follows, move the slider farther along, and make the current again.

With careful work, it will be shown that with 
descending currents; the first contraction will be on closure only. With ascending currents, the first contraction will be on opening the current.

The explanation is that, with currents which pass through the sartorius from end to end the point of greatest density is the smaller, lower end. This is cathodal in descending currents, anodal in ascending currents.

Effect of the Angle at which the Current Lines cut the Muscle Fibres

Connect non-polarizable electrodes through a key with a dry cell. Build on a glass plate with normal saline clay two parallel walls a little longer than the sartorius muscle and one centimetre apart. Join the ends with wax, to make a rectangular trough. Remove a sartorius muscle from a curarized frog, avoiding all injury to the muscle. Place the muscle in the trough, and cover it with normal saline solution. Bring a non-polarizable electrode against the centre of each long side, so that the current lines shall cut the muscle fibres at right angles. Close the key.

There will be no contraction. The muscle is inexcitable to currents that cross its fibres at right angles. 
Alter the angle by moving one electrode to the right, the other to the left, and repeat the experiment.

The stimulating effect will increase as the angle between current lines and the long axis of muscular fibres diminishes.

Nerves also are inexcitable to transverse currents. Differences in resistance play a great part here. The resistance of nerves is said to be $2 \frac{1}{2}$ million times that of mercury, when the current passes along the nerve, and $12 \frac{1}{2}$ million times when it passes transversely.

\section{The Induced Current}

The break induction current, owing to its rapid rise from zero to maximum intensity, is a more effective physiological stimulus than the rnake current, and may therefore be chosen for experimentation.

1. The direction of the induction current in the secondary coil is most easily determined electrolytically.

Arrange the inductorium for maximal currents. Bring wires from the posts on the secondary coil to a piece of filter paper wet with starch paste containing iodide of potassium. Exclude the make currents with the short-circuiting key; 
pass the maximal break currents through the electrolyte.

Iodine will be set free at the anode and will combine with the starch to form blue iodide of starch.

Mark the positive post on the secondary coil with a plus sign.

2. Connect the poles of the secondary coil through a pole-changer with non-polarizable electrodes. Make a nerve-muscle preparation. Tie a ligature about the nerve about two centimetres from the central end. Place one electrode on each side of the ligature. The passage of a nerve impulse from the central electroale to the muscle will be prevented by the ligature, although the electric current can still pass between the electrodes. Turn the polechanger so that the electrode on the peripheral (muscle) side of the ligature shall be first the anode and then the cathode, and test the irritability to weak induction currents, beginning with the secondary coil some distance from the primary, and gradually increasing the intensity.

Only cathodal stimulation will produce contraction. The same result can be secured by separating the cathode and anode with ammonia. If the nerve is painted with ammonia in the intrapolar region, break currents cease to cause 
contraction when the cathode is on the central side of the painted zone. Painting the cathodal region directly also prevents excitation.

The failure of the induction current to stimulate at the anode, on opening the current, is due to the exceedingly brief duration of the induced current; there is not time for a sufficient anelectrotonic alteration in excitability. If the current is shortened still more (if it be less than 0.0015 sec.), the cathodal excitation also disappears. With very strong currents, however, opening the current stimulates as well as closure.

3. Additional evidence of polar action is secured by connecting the electrodes with the capillary electrometer through a closed shortcircuiting key. The meniscus is brought into the field, the nerve is stimulated repeatedly with maximal break currents, and then stimulation is stopped, and the short-circuiting key in the electrometer circuit. opened. The meniscus will move in a direction indicating a higher potential at the anode (positive anodal polarization current).

4. Finally, it may be added that the galvanic current may increase the stimulating effect of the induced current as pointed out on page 80 , but only when the cathode of the induced current falls in the cathodal region of the polarizing current. 
The law of polar excitation holds good then for the induced as well as the galvanic current. In fact, there is no essential difference between the physiological effects of induced currents and very brief galvanic currents.

Increasing the intensity of the induced current increases at first the excitation (height of contraction). At length, however, with ascending currents, a point is reached beyond which further increase in strength is followed first by the diminution and at length by the disappear ance of contraction. With still higher intensities, the contractions reappear. This gap in the contraction series is explained by the increasing depression of irritability at the anode blocking the cathodal impulse; when the intensity is still further increased, the opening of the current acts as a stimulus. A similar result may be secured with the galvanic current.

\section{Apparatus}

Normal saline. Bowl. Pipette. Towel. Simple key. Non-polarizable electrodes. Nerve holder. Potter's clay mixed with 0.6 per cent solution of sodium chloride. Saturated solution of zinc sulphate. Muscle clamp. Stand. 13 wires. Kymograph. Glazed paper. Two muscle levers. Thread. Rheochord. Two dry cells. Moist chamber. Glass plate. Ice. Paraffin paper. Cork clanip. Pole-changer. Beaker. Tripor, Sodium chloride. 


\section{GENERAL PROPERTIES OF LIVING TISSUES}

Inductorium. Electrodes. Bunsen burner. Intestine of a rabbit. Electromagnetic signal. Tuning fork. Brass electrodes. Fine copper wire. Frog board. 2 pairs of metal electrodes, each passed through cork. Electrometer. Paramecia. Microscope. Glass slide. Bent hooks. One per cent solution of veratrine acetate. Fine glass pipette. Filter paper saturated with starch paste containing potassium iodide. Frogs. Fine rubber tubing for insulating electrodes. Ammonia. One per cent solution of acid potassium phosphate. Two per cent solution of sodic carbonate. Ligatures. Filter paper. 
CHEMICAL AND MECHANICAL STIMULATION 163

\section{V}

CHEMICAL AND MECHANICAL STIMULATION

Chemical Stimulation

THE contractility, heat production, and other phenomena of the life of muscle rest at base on chemical processes. Anything that sufficiently alters these processes may be a stimulus. A most important source of stimulation is the alteration of the chemical composition of muscle through osmosis.

Effect of Distilled Water. - Place a sartorius muscle in distilled water.

Irregular contractions usually occur. The muscle soon sweils, and becomes white, turbid, cadaveric.

These striking changes depend on the withdrawal of certain bodies by osmosis. Muscle contains large quantities of proteid, particularly proteids of the globulin class; certain carbohydrates, such as glycogen; nitrogenous and other extractives; water; and a number of inorganic salts. Most of these bodies are largely or wholly insoluble in water, and require for their solution the presence of inorganic salts. 
The globulins, for example, are insoluble in distilled water, but soluble in dilute solutions of sodium chloride. The osmosis of salts into the distilled water in the above experiment first stimulates and then destroys the contractility of the muscle.

An increase in the saline content of the muscle juice or "plasma" also acts as a stimulus, and, if excessive, may be fatal.

Strong Saline Solutions. - Place a sartorius muscle on a slightly inclined glass plate. Cover the lowest fourth of the muscle with crystals of sodium chloride.

Irregular contractions will appear.

Drying. - The effect of loss of water is best shown in nerve.

Let the nerve of a nerve-muscle preparation dry. Note the twitching of the muscle as the water content diminishes. Test the irritability of the nerve from time to time with induction currents. It will first increase, then disappear as the nerve dries.

Wet the nerve with 0.6 per cent sodium chloride solution.

The irritability will reappear.

To keep muscles and nerves in good condition for experimentation, it is necessary to moisten them with a solution containing the inorganic salts most abundant in the tissue-liquids in the 
proportions in which they are present in those liquids. Practically, a 0.6 per cent solution of sodium chloride has commonly been employed, in the case of the frog. Such a solution is said to be isotonic, $i$. $e$. neither giving nor taking water from the tissue. That it is not perfectly indifferent appears from this experiment.

"Normal Saline." - Allow a sartorius muscle to stand half an hour in normal saline solution ( 0.6 per cent $\mathrm{NaCl})$. Record its contraction in response to a maximal break induction current. In place of a simple twitch, a prolonged contraction of abnormal height and duration will usually be secured.

Importance of Calcium. - Place the "normal saline" sartorius in 0.6 per cent sodium chloride solution containing 10 per cent of saturated solution of calcium sulphate. After ten minutes record the maximal break contraction.

The abnormal contraction will have disappeared.

Constant Chemical Stimulation may cause Periodic Contraction. - Place a sartorius muscle in a solution of 5 grams $\mathrm{NaCl}, 2$ grams $\mathrm{Na}_{2} \mathrm{HPO}_{4}$, and 0.4 gram $\mathrm{Na}_{2} \mathrm{CO}_{3}$ in one litre of distilled water.

Usually rhythmic contractions are seen. All contractile substance shows a tendency to periodic contractions in response to a constant stimu- 
lus, whether chemical, mechanical, or electrical. There are reasons for believing that the rhythmical contractious of the heart are the consequence of a constant chemical stimulus.

\section{Mechanical Stimulation}

Stimulate a nerve mechanically by pinching the cut end with forceps.

No change will be seen in the nerve, but the muscle will shorten, and then relax.

Mechanical stimulation has the advantage that it can be localized accurately, and for this reason it has been used where electrical stimulation seemed inapplicable. Tetanornotors have been constructed by Heidenhain and others to give a rapid succession of slight blows upon the nerve.

Sudden pressure on a muscle or sudden extension may cause contraction. Sometimes the whole muscle contracts, sometimes only the portion directly stimulated.

Idio-Muscular Contraction. - With the point of the seeker stroke the diaphragm and other muscles of a recently killed rat, or other small warm-blooded animal, in a direction at right angles to the course of the fibres.

$A$ wheal, $i$. e. a long-continued shortening and thickening of the fibre stimulated, will be seen. If the animal be not too long dead, a momentary 
twitch of the whole of the fibre stimulated will precede the continued local contraction or wheal.

The same phenomenon is seen for a briefer time on sharp mechanical stimulation of muscles in living animals, for example, the wheals raised by the blow of a whip. In men long ill of wasting diseases, $e . g$. phthisis, the idio-muscular contractions appear on drawing a pencil point across the muscles. Direct total stimulation of frog's muscle, especially in the spring months, may be followed by long continued contraction. Fatigue, cold, and many poisons, such as veratrine, favor the prolongation of the phase of shortening. The idio-muscular contraction is not a "tetanus," i. e. not a prolonged shortening due to successive contractions, the interval between which is too short to permit of relaxation, but a prolonged single contraction, the cause of which lies in the muscle and not in the nerve.

\section{Apparatus}

Normal saline. Bowl. Pipette. Towel. Glass plate. Distilled water. Sodium chloride. Solution of sodium chloride ( 0.6 per cent), containing 10 per cent of saturated solution of calcium sulphate. Solution containing 5 grams sodium chloride, 2 grams di-sodium hydrogen phosphate, and 0.4 gram sodium carbonate, in 1000 c.c. water. Small warm-blooded animal recently killed. Introduction coil. Dry cell. Key. Electrodes. 3 Wires. Frogs. 


\section{VI}

\section{IRRITABILITY AND CONDUCTIVITY}

IRRITABILITY is the power of discharging energy on stimulation. The form in which the kinetic energy of muscle appears is partly mechanical work (the visible contraction) and partly molecular, - heat, chemical action, and electricity. In the nerve, the kinetic energy is wholly molecular; an electromotive force is generated, probably heat is set free (though this statementwhich is based simply on analogy - is frequently disputed), and a molecular change - the nerve impulse - arises at the seat of stimulation. In both muscle and nerve, by virtue of their conductivity, the change induced by stimulation is as a rule not limited to the region stimulated, but passes in both directions along each stimulated fibre. In neither muscle nor nerve can the changes in energy spread transversely; they are limited to the muscle- or nerve-fibre in which they arise.

It will be shown that conductivity and irritability are essentially different functions. 
The Independent Irritability of Muscle. - The stimulus that causes the contraction of a muscle may be applied either to the nerve or to the muscle itself. If to the nerve, the muscle will be thrown into the active state not by the original stimulus, but by a nerve impulse. If to the muscle, the nerve will still be stimulated, for examination shows terminal fibres distributed, in skeletal muscle at least, probably to every fibre, and with few exceptions to all parts of the muscle. The fact that muscles may contract when an electric current flows through them, or when otherwise stimulated, does not therefore of itself indicate that electricity is a stimulus to muscle protoplasm. Before this can be established, it will be necessary to demonstrate contraction in parts of muscle not provided with nerves; for example, the distal part of the sartorius, or in muscles in which the nerves have been destroyed by curare or by degeneration.

Nerve-free Muscle. - Remove the sartorius muscle, together with the portion of the pelvis and the tibia to which the muscle is attached, and lay it on a glass plate. Stimulate the distal (tibial) fifth, in which examination with the microscope would show the absence of nerve fibres, with a strong break induction current.

The nerve-free muscle will contract. 
Muscle with Nerves Degenerated. - A nerve fibre severed from its cell of origin dies or "degenerates" down to its ultimate endings. Expose the sciatic nerve in the middle of the thigh of a frog in which the nerve has been severed near the pelvis ten days before, so that the whole of the nerve distal to the section shall have degenerated. Stimulate the degenerated trunk.

No contraction is seen in the muscles of the leg. Stimulate the muscles directly.

Contraction takes place.

The Nerve-free Embryo Heart. - Embryological studies show that the nerves of the heart are formed from epiblast in the walls of the neural canal, and do not grow into the heart until the close of the third day of incubation (chick). The heart, however, begins to beat during the second day of embryonic life, before even the blood which it shall pump is formed. Thus the heart muscle, in the embryo, is capable of contraction in the absence of nerves.

Cover an egg which has been incubated 60-70 hours with 0.75 per cent solution of sodium chloride warmed to $38^{\circ} \mathrm{C}$. Remove the shell with the forceps over one third of the egg, begimning at the broad end, and leaving the shell membrane behind. Now remove the shell membrane. Note the beating heart. 
Paralysis of Nerve Endings with Curare.Make two nerve muscle preparations $\mathrm{A}$ and $\mathrm{B}$, and fill two watch glasses with curare solution. In one watch glass lay the nerve trunk of preparation $\mathrm{A}$ and in the other watch glass the muscle of preparation B. Cover muscle A and nerve B with filter paper moistened with normal saline solution, to prevent drying. At intervals of ten minutes stimulate nerve $\mathrm{B}$ with induction currents.

When the poison has acted the stimulation of nerve $B$ will produce no contraction of the attached muscle, which lies in the curare. Either the muscle or the nerve has been poisoned.

Stimulate muscle B directly.

It contracts. Hence the curare has poisoned the nerve; probably the terminals of the nerve within the muscle.

Now remove nerve $A$ from the curare and stimulate the trunk of the nerve.

The attached muscle will contract. Hence the trunk of the nerve has not been poisoned by the curare.

It follows that curare poisons the endings of the nerve within the muscle. Therefore, the contraction of muscle $\mathrm{B}$, in which the nerve endings were paralyzed, must have been due to the independent irritability of the muscle fibres. 
The occurrence of idio-muscular contraction (see page 166) is an additional proof of the independent irritability of muscle.

Irritability and Conductivity are Separate Properties of Nerve. - 1. Carbon-dioxide. - Arrange the inductorium for tetanizing currents. Connect the secondary coil with the main posts of the pole-changer (cross-wires out). Connect the

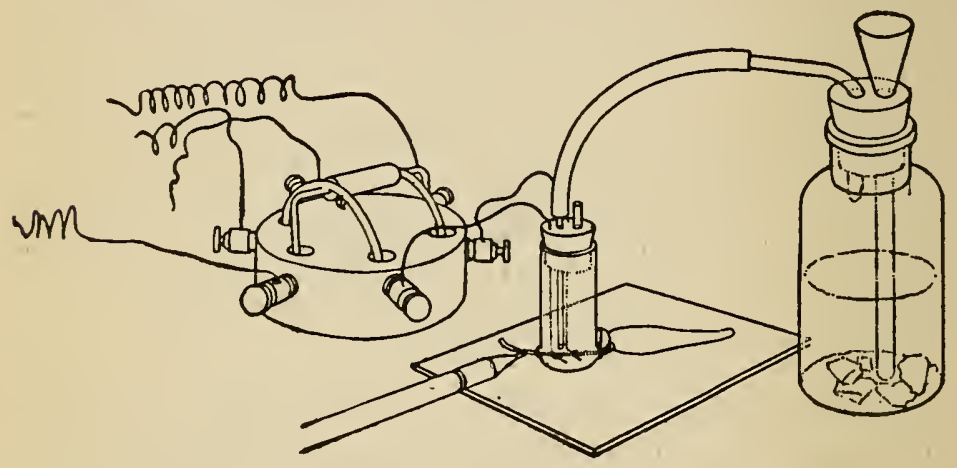

Fig. 42. The gas chamber, with bottle for generating carbon dioxide, and a pole-changer arranged to stimulate the nerve either within or without the ehamber. The holes in the glass through which the nerve passes are plugged with normal saline clay.

two other pairs of posts with the usual stimulation electrodes and the electrodes of the small gas chamber (Fig. 42). Join the inflow tube of the gas chamber with the outflow tube of the carbon-dioxide bottle. The gas chamber should rest on a glass plate. Make a nerve-muscle preparation, preserving the full length of the sciatic nerve up to the vertebral column. Tie 
a silk thread to the extreme end of the nerve; and fasten the thread to the end of the seeker by a drop of wax cement. With the aid of the seeker, pass the thread through the holes, and draw the nerve after, so that the nerve lies on the electrodes. The nerve should be drawn through until the muscle is close to the gas chamber. Stop the holes through which the nerve passes with normal saline clay. Bring the outer pair of electrodes against the central end of the nerve near its exit from the gas chamber. Determine which position of the pole-changer corresponds to each pair of electrodes. Stimulate the nerve first within the chamber, and then on the central end of the nerve, using a current just sufficient to cause tetanus. In both cases tetanus will result. Now pour 20 per cent hydrochloric acid on the marble in the generator. After the gas has passed through the chamber for a moment, stimulate as before.

Stimulation of the portion of the nerve exposed to the carbon-dioxide is no longer effective, while stimulation of the part central to the gas chamber still produces tetanus.

But the nerve impulses created by stimulation of the nerve central to the gas chamber cannot reach the muscle except by passing along the nerve and through the carbon-dioxide. The con- 
ductivity of the nerve therefore is still sufficient, while the irritability has been suspended by the action of the gas. Hence, conductivity and irritability are by no means interchangeable terms.

Their essential difference is further shown by the effect of alcohol vapor, which impairs conductivity while irritability is little changed.

2. Alcohol. - Disconnect the rubber tube from the gas generator, and blow through the gas chamber until the carbon-dioxide is driven out. The nerve will recover its irritability. Determine this by stimulating from time to time. When the nerve has recovered, drop a little alcohol through the long glass tube of the gas chamber, being careful that only the vapor of the alcohol comes into contact with the nerve. Stimulate both within and central to the chamber.

After a time, tetanus will no longer be produced by stimulating central to the chamber. Stimulation within the latter is still effective. Thus conductivity is impaired, while irritability remains intact, or at least is affected to a less extent. (The electrodes within the alcohol atmosphere should not be too far from the opening through which the nerve passes to the muscle, else the loss of conductivity in this part of the nerve may make difficult the demonstration of irritability.) 
Minimal and Maximal Stimuli ; Threshold Value. - Arrange the gastrocnemius muscle to write on a smoked drum. Connect one binding post of the secondary coil to the muscle clamp, the other binding post to the post on the muscle lever. Load the muscle with 10 grams. Describe an abscissa on the smoked paper, turning the drum by hand. Send a feeble break induction current through the muscle.

There will be no response.

Repeat the break currents, gradually moving the secondary closer to the primary coil.

At a certain point the muscle will just contract ("threshold value"). This is a minimal contraction produced by a minimal stimulation.

Turn the drum $5 \mathrm{~mm}$., move the secondary coil $5 \mathrm{~mm}$. nearer the primary, send in another break current, and record the contraction. Continue this.

The contraction in answer to each break curreut increases with the strength of the currents at first rapidly, then slowly, up to a certain point. Further increase in the strength of the stimulus produces no further increase of contraction. The stimulus and the resulting contraction have now become maximal.

There is a striking disproportion between the energy of the stimulus necessary to throw a 
nerve or muscle into the active state, and the energy that the stimulus sets free. It is as if a spark fell into powder; the active process is to be regarded, with some reservations, as an explosion. But only a part of the latent energy of muscle can be set free by any one stimulus.

Threshold Value Independent of Load. - Repeat the preceding experiment, and load the muscle with 50 grams instead of 10 .

The threshold value will not be changed.

\section{Summation of Inadequate Single Stimuli. -} Place the secondary coil of the inductorium at such a distance from the primary that a break current shall be nearly, but not quite sufficient to cause a contraction. Let the muscle rest without stimulation for about a minute. Repeat the inadequate single stimulation at intervals of five seconds. No curve need be written.

After a time, contraction will be secured.

The excitation outlasts the stimulus, and reinforces subsequent stimuli: finally, the summed excitations call forth a contraction. Summation is of frequent occurrence probably in all living tissues.

Relative Excitability of Flexor and Extensor Nerve Fibres; Ritter-Rollett Phenomenon. - Expose the sciatic nerve in a brainless frog in the pelvic region. Set the hammer of the in- 
ductorium in action (binding posts 2 and 3), and stimulate the nerve with weak induction currents.

The leg will be flexed.

Use stronger induction shocks.

As the intensity increases extension as well as flexion is seen. A still further increase causes extension only.

The gradations of intensity necessary to show these results are sometimes difficult to secure. The phenomenon of relative excitability is not limited to the case just cited. Weak stimulation of the vagus causes adduction of the vocal bands; stronger stimulation, abduction. Weak stimulation causes opening of the claw of the lobster, while stronger stimulation of the same nerve causes closure. Weak stimulation of the hypoglossal nerve in the dog and rabbit causes the tongue to be thrust from the mouth, while with strong stimulation the tongue is withdrawn into the mouth. It must not be forgotten that the anatomical nerves stimulated in these experiments are composed of many axis cylinders, each of which is a physiological nerve. That they should vary in excitability is to be expected.

A second and probably better explanation of the Ritter-Rollett phenomena is found in the difference in structure of the flexors and extensors. 
Muscle fibres consist of contractile substance inbedded in sarcoplasm. The relation between the contractile substance differs in the same muscle in different species and individuals, and differs further in the muscles of the same individual. In striated muscles of vertebrates, those rich in sarcoplasm have a turbid, opaque appearance, while those poor in sarcoplasm are translucent. Important differences in contractility, irritability, etc., depend on this difference of structure. Muscles which contain many "clear" fibres (poor in sarcoplasm) are more irritable than those containing many of the fibres rich in sarcoplasm. In the flexors of the frog the "clear" fibres are relatively more numerous than in the extensors.

Specific Irritability of Nerve Greater than that of Muscle. - Arrange an inductorium for single induction currents. Make as rapidly as possible two nerve-muscle preparations, A and B. Bring a wire from the secondary coil to each end of muscle $A$. Let the nerve of $B$ rest on muscle $A$. No stimulation can now reach $B$ except through that part of the nerve of $B$ which rests on muscle A. Place the secondary some distance from the primary coil. Stimulate muscle A with make induction shocks, the strength of which is gradually increased by approximating the coils. 
Muscle $B$, which is stimulated only through its nerve, will contract before muscle $A$, which is stimulated directly. Hence, the specific irritability of nerve is greater than that of muscle, provided (1) that the intensity of the stimulating current is equal for both nerve and muscle, and (2) that the irritability of the two muscles does not differ, and (3) that the stimulation of the nerve of $B$ is not by unipolar induction. The first source of error may be excluded, becanise the density of the current passing through the portion of nerve lying on muscle $A$ is certainly not greater than the density of the current passing through the muscle itself. The second possibility is tested as follows:-

Reverse the muscles and repeat the experiment.

The result will not be altered.

The third source of error is excluded as follows.

Tie a ligature about the nerve of $\mathrm{B}$, between muscles $A$ and $B$. The physiological conductivity of nerve $\mathrm{B}$ is thereby destroyed, and the nerve impulse cannot pass; but the physical continuity of the nerve, and hence its power to conduct electricity, is still present.

The strongest induction currents applied to muscle A will now fail to produce contraction of $\mathrm{B}$. 
Irritability at Different Points of Same Nerve. Determine the threshold value for the sciatic nerve near the gastrocnemius muscle and about two centimetres from the cut end of the nerve.

The farther from the muscle the nerve is stimulated, the lower will be the threshold value. It has been suggested in explanation of this that the nerve impulse gathers force as it passes along the nerve, and is the more powerful the longer the nerve which it traverses (avalanche theory). It has also been suggested that the nearer to the nutrient cell of origin the stimulus is applied, the greater the effect. The true explanation lies in the fact that the irritability of the nerve is raised in the neighborhood of the cross-section by the passage of the demarcation current through that portion, as explained on page 296. Tigerstedt has shown with mechanical stimuli that the uninjured nerve has equal irritability throughout.

The Excitation Wave remains in the Muscle or Nerve Fibre in which it starts. - In order to limit the stimulus to one or two fibres, the method of unipolar stimulation may be adopted.

Fasten in one post of the secondary coil of the inductorium arranged for tetanizing currents a wire soldered to a blunt needle. The needle, except near the free end, and the lower part of 
the connecting wire, should be inclosed in a glass tube for insulation.

Expose the sacral plexus in a brainless frog in which the skin has been removed from the hind limbs. Connect the preparation by means of a copper wire with the earth through the gas or water pipes.

Touch the sacral nerves here and there with the needle electrode, watching meanwhile the sartorius muscle.

Partial contractions will be seen in the sartorius, now of the inner, now the outer fibres, according to the nerve fibres touched

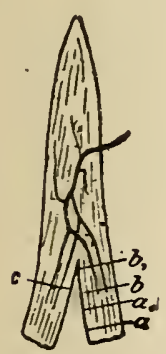

Fig. 43. The sartorius. by the needle.

Stimulate the sartorius directly.

Only the fibres touched by the needle contract.

Evidently the excitation wave remains limited both in the muscle and the nerve to the fibres in which it starts.

The same Nerve Fibre may conduct Impulses both Centripetally and Centrifugally. -1 . The nerve of the sartorius divides at the muscle, part going to each half of the muscle (Fig. 43). Microscopical examination shows that the division is not simply a parting of individual nerve fibres, but that each axis cylinder forks, one 
limb going upwards, the other downwards. If the muscle be severed between the forks, no impulse started in one half of the muscle could reach the other half, except by going up one branch to the original axis cylinder and down the remaining branch; for it is known that the nerve impulse does not escape transversely from one axis cylinder to other neighboring ones.

Remove a sartorius muscle with great care. Split the muscle in the middle line for one third of its length, beginning at the broad end, as indicated in the diagram. Stimulate the muscle fibres of the right segment mechanically, by snipping the preparation with scissors in the line $a$. Do not cut quite through the segment.

Only the right half twitches.

Repeat the stimulus by snipping in the line $\alpha_{1}$. Again only the right half twitches.

Stimulate in the line $b$.

Both segments twitch, or at least some fibres in each.

Repeat at $b_{1}$.

Both segments twitch again.

2. The gracilis of the frog is divided into an upper, shorter part and a lower, longer part by a tendon (Fig. 44, j). Each axis cylinder in the nerve $N$, on approaching the muscle, divides into two branches, one of which goes to the 
upper and the other to the lower portion of the muscle.

Remove the muscle together with a portion of its attached nerve, and examine the inner surface (Fig. ,44). The nerve $(N)$ divides into two branches, of which the upper $(K)$ rums to the shorter portion of the muscle and is unbranched for some distance, while the other $(L)$ has a very short stem and sinks almost at once into the

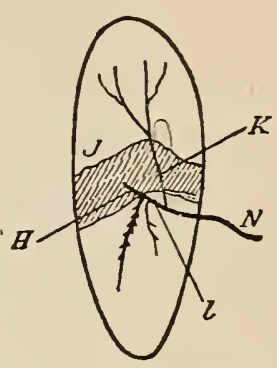

Fig. 4t. The gracilis. substance of the lower part. One of the branches (H) perforates the muscle and goes to the skin.

With a sharp pair of scissors cut out entirely the part shaded in the diagram, without injuring the nerves. The halves of the muscle are now united only by the

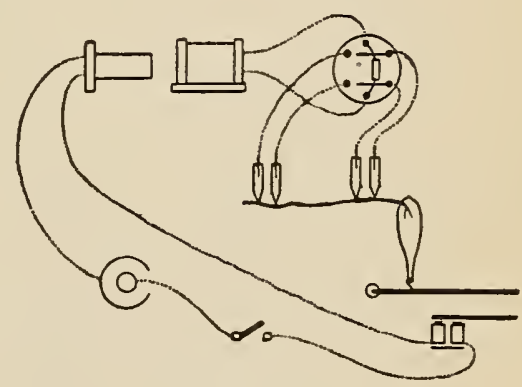

Fig. 45. forked nerve.

Stimulate the end branches of the nerve in one of the pieces of muscle by snipping with scissors; also chemically, with a lump of salt.

Both pieces will contract. 
Speed of Nerve Impulse. - Smoke a drum, and adjust it for "spinning." Place two pairs of needle electrodes in corks in the moist chamber. Arrange the inductorium for maximal make currents, placing a simple key and the electromagnetic signal in the primary circuit (Fig. 45). Connect the secondary coil to the side cups of the pole-changer. Connect the end pairs of cups each with one pair of the electrodes in the moist chamber. Make a nerve-muscle preparation, preserving the full length of the sciatic nerve. Fasten the femur in the clamp in the moist chamber. Connect the Achilles tendon to the muscle lever. Bring the point of the lever against the drum immediately over the writing point of the electro-magnetic signal. Let the nerve rest on the electrodes, one pair near the end of the nerve, the other near the muscle. Spin the drum slowly. Hold the writing point of a vibrating tuning fork against the smoked paper beneath the line drawn by the signal. Send a maximal induction current through first one pair of electrodes and then the other. Determine the interval between the moment of stimulation and the beginning of contraction in each instance. [This is done by turning the drum back until the writing point of the signal lies precisely in 
the vertical line marked by it when the current was made, and then stimulating the muscle to contract. The ordinate drawn by the muscle lever (the drum being still at rest) will be synchronous with the ordinate drawn by the signal during the experiment.]

It will be found that the interval between stimulation and contraction is greater when the nerve is stimulated far from the muscle than it is on stimulation near the muscle. The difference is the time occupied by the passage of the excitation wave along the nerve between the electrodes.

Measure the length of nerve between the electrodes, and calculate the speed of the nerve impuise per second.

It is assumed in this method that the interval between the closure of the primary circuit and the beginning of the nerve impulse is the same in both instances, and that the interval between the arrival of the impulse in the muscle, and the visible change of form, is likewise the same in both. If the mean and the probable deviation of a series of measurements are taken, a fairly accurate result may be expected. A better method, however, is to record the passage of the negative variation over a measured length of nerve by photographing the meniscus of the capillary 
electrometer. Similar measurements can be made with a differential rheotome (page 313 ).

Helmholtz found in motor nerves of the frog an average speed of 27 metres per second, but the individual variation is considerable. The speed is very slow compared with that of light, or even sound. It is modified by changes in temperature, nutrition, anæesthetics (alcohol, ether, chloroform, carbon dioxide), the intensity of the stimulus, - above a certain value, the greater the stimulus, the more rapid the conduction, and by many other factors. Specific differences are found depending on the structure of the nerve. Thus the velocity has been found in mammalian nerve to smooth muscle to be about 9 metres per second, while in the bivalve Anodonta it is said to be only 1 centimetre per second.

\section{Apparatus}

Normal saline. Bowl. Towel. Pipette. Glass plate. Dry cell. Inductorium. Key. Wires. Frog with sciatic nerve degenerated. Hen's eggr incubated 60-70 hours. $\mathrm{NaCl}$ solution $(0.75 \%)$. Ligatures. Filter paper. One per cent solution of curare. Pole-changer. Gas chamber. Carbon dioxirle generator. Twenty per cent hydrochloric acid. Broken marble. Alcohol. Muscle elamp. Stand. Mnscle lever with seale pan. Millimetre rnle. Ten gram weights. Neerlle electrorles (glass tuhe). Moist chamber. Two pairs of non-polarizable electrorles. Electro-magnetic signal. Recording drum. Glazed paper. 'Tuning fork. Normal saline elay. 


\section{PART II}

\section{THE INCOME OF ENERGY}





\section{P A R T I I}

\section{THE INCOME OF ENERGY}

\section{FERMENTATION}

\section{Hydrolysis of Starch by Diastase}

\section{Conversion of Starch to Sugar by Germinating}

Barley. - To 5 grams crushed, germinating barley add 10 grams potato starch, and 20 c.c. of cold water. Then add gradually 70 c.c. of hot water with constant stirring. Keep the mixture in a temperature of about $60^{\circ} \mathrm{C}$. for one hour.

The insoluble starch will be converted to a sweet liquid. ${ }^{1}$

Boil 10 c.c. of Fehling's solution, ${ }^{2}$ dilute the syrup with water and add it drop by drop to the boiling Fehling's solution.

1 Kirchoff : Schweigger's Journal für Chemie und Physik, 1815 , xiv, p. 389. There is a small amount of sugar and starch in the barley itself.

2 Fehling's solution. - In a large watch glass weigh 34.639 gms. pure cupric sulphate (clean crystals). Dissolve the crystals by warming them with about 150 c.c. water in an evaporating dish. Place the solution in a 500-c.c. measuring flask. Wash the remnant from the dish into the flask. Allow the liquid to cool completely. Add water to the mark on the neck of the flask.

Warm about 173 gms. potassium sodium tartrate in a little water until dissolved. Place the solution in a 500-c.c. measuring flask, add 100 c.c. sodium hydroxide, sp. gr. 1.34 (about 31 per cent), and, after the mixture has completely cooled, fill the flask to the mark on the neck.

In use, mix equal volumes of each solution in a dry glass.

One molecule grape sugar reduces five molecules cupric oxide to cuprous oxide ; 10 c.c. of Fehling's copper sulphate solution equals $0.05 \mathrm{gm}$. grape sugar. 
Red cuprous oxide or its yellow hydrate will separate.

The germinating barley causes the starch to take up water, thus changing to a reducing sugar. In this instance the agent is a living cell, or some substance or "ferment" secreted by the cell. It is now necessary to inquire whether ferments are separable from living cells.

Conversion of Starch to Sugar by Salivary Diastase (Ptyalin). - To 10 c.c. of starch paste ${ }^{1}$ colored blue with iodine (blue iodide of starch) add about 2 c.c. of filtered saliva and keep the mixture at $35-40^{\circ} \mathrm{C}$.

The starch paste will liquefy and become sweet. The blue color will become lighter and finally disappear.

Test with Fehling's solution. Reduction will take place.

Saliva hydrolyzes starch to a reducing sugar.

Saliva is secreted by the cells in the salivary gland, placed some distance from the mouth. The saliva itself contains no secreting cells. There are ferments, then, which act at a distance

1 Starch paste. - Rub $1 \mathrm{gm}$. potato stareh in a mortar with 25 c.c. cold water. I'our the mixture into an evaporating dish. Wash the remnant from the mortar and pestle into the dish with 75) c.e. water. Heat the mixture to boiling point with constant stirring. The stareh paste will turn blue upon addition of iodine (iodide of starch). 
from the cells that produce them. There seems thus an important distinction to be made between organized ferments, those acting apparently within the living cell, and unorganized ferments, like the salivary diastase, which is secreted by a living cell but remains active after leaving the cell. It will be seen that this distinction cannot be maintained.

Extraction of Diastase from Germinating Barley. ${ }^{1}$ - Crush freshly germinating barley in a mortar with about half its weight of water. Keep the mass two hours at $35-40^{\circ} \mathrm{C}$. Squeeze out the watery extract in a press, or strain by strong pressure through a linen cloth. Add excess of alcohol.

Diastase will be precipitated. It may be purified by dissolving it in water and reprecipitating with alcohol.

Add a little diastase to 10 c.c. starch paste, colored blue with iodine. The starch will be converted to sugar. The blue color will disappear.

It appears, therefore, that ferment action is not dependent on the life of the cell that secretes the ferment.

Specific Action of Ferments. - The question now arises whether the diastase acts only to

1 Payen and Persoz: Annales de chimie et de physique, 1833, liii, p. 78. 
change starch to sugar or whether it causes the decomposition of other substances.

Place a small piece of fibrin in a test-tube and add 2 c.c. filtered saliva. Keep the tube several hours at a temperature of $35-40^{\circ} \mathrm{C}$.

The fibrin will not change.

Place 0.5 c.c. nentral olive oil (page 207) and 2 c.c. filtered saliva in a test-tube.

Noteworthy changes will be absent.

From these experiments it is evident that diastase decomposes starches, but does not decompose proteids and fats. Its ferment action is thus far "specific." The belief that each ferment has its own characteristic product will be increased by the study of the following typical ferment actions.

\section{Proteid Digestion by Pepsin}

Gastric Digestion of Cooked Beef and Bread. At 7 A.M. feed cooked beef and bread to a cat which has fasterl twelve hours. At 11 А. M. kill the cat, expose the stomach, and apply double ligatures about $1 \mathrm{~cm}$. apart to the duodenum at the pylorus and to the oesophagus at the cardiac orifice. Remove the stomach. Open the stomach very cautiously by drawing a knife along the greater curvature.

"The stomach is very full, and still contains 
inuch meat and bread not wholly softened. The softening is greater in the portal region and in those portions of the food next the mucous membrane than in the middle of the stomach contents. The mucus secreted by the gastric mucous membrane is very abundant and is strongly acid. The stomach contents have a sour odor." 1

Artificial Gastric Juice. ${ }^{2}-1$. Strip the mucous membrane from the fourth stomach of a calf. Wash the membrane with cold water until the acid reaction disappears. Dry the mucous membrane in the air. Divide some of the dried membrane into small pieces and add dilute hydrochloric acid. ${ }^{3}$

2. Strip the mucous membrane of the pig or rabbit from the deeper layers of the stomach, cut the mucous membrane into the smallest pieces, wash slightly with water, pour off the water with all possible care, and cover the slightly moist residue with glycerine. ${ }^{4} \quad$ Before using, add dilute hydrochloric acid.

1 Eberle : Physiologie der Verdauung, 1834, p. 100.

2 Eberle : loc. cit., p. 79.

3 Dilute hydrochloric acid. - Add to 10 c.c. officinal $\mathrm{HCl}$, sp. gr. 1.124 (about 25 per cent $\mathrm{HCl}$ ), enough water to make 1000 c.c. This solution will contain about 0.281 per cent $\mathrm{HCl}$. (Salkowski's Practicum, 1893, p. 130.)

4 Von Wittich: Archiv für die gesarnmte Physiologie, 1869, ii, p. 194. 
Digestion with Artificial Gastric Juice.-Prepare three flasks, $A, B$, and $C$. In $A$ place 100 c.c. artificial gastric juice; in $B, 100$ c.c. 0.2 per cent $\mathrm{HCl}$; and in $C$, a piece of dried gastric membrane and 100 c.c. distilled water. In each of the three flasks place a small piece of cooked meat, and keep the flasks about five hours at $35-40^{\circ}$ C. ${ }^{1}$ Compare the result with that observed in natural digestion.

The artificial gastric juice will digest the meat as did the natural juice in the stomach, but neither the acid alone, nor the mucous membrane free from acid, will digest. There is a ferment in the mucous membrane, but it will not act except in an acid medium.

Extraction of Pepsin. - Pepsin more or less contaminated with proteid (pepsin may itself be a proteid) may be precipitated from a glycerine extract by alcohol. ${ }^{2}$ The pepsin may also be carried down mechanically by an indifferent precipitate as in Brūcke's method, ${ }^{3}$ in which the nucous membrane, acidulated with phosphoric acid, is allowed to digest until the proteids are mostly converted into soluble peptone. The mixture is then neutralized with lime water. The insoluble calcium plosphate thus formed falls as

1 Eherle : loc. cit.

2 Von Wittich: loc. cit., p. 195.

3 Briicke: Sitzungsherichte rler königliche Akadęmio der Wissenschaften zu Wien, 1862, xliii, 1. 601. 
a fine powder carrying the pepsin with it. The precipitate is dissolved in very dilute hydrochloric acid, and to this solution is added a solution of cholesterin in alcohol and ether. When the two solutions are mixed, the cholesterin separates as an abundant, fine powder bearing the pepsin with it. The cholesterin is removed with ether, leaving the pepsin.

Ammonium sulphate may also be used as the mechanical precipitant. ${ }^{1}$

Change of Proteid to Peptone by Pepsin. - 1 . Place in a test-tube five drops of the glycerine extract of pepsin with 5 c.c. 0.2 per cent hydrochloric acid and a small piece of fibrin.2 Keep the mixture at $35-40^{\circ} \mathrm{C}$.

In a short time the fibrin will be dissolved. Appropriate tests will show that it has been converted to peptone. 2. Repeat the preceding experiment, using commercial pepsin (never very free from proteid).

\section{Sylitting of Casein by Rennin.}

Rennin Extract. - Allow the mucous membrane of the stomach (preferably the fourth stomach of

1 Kühne and Chittenden: Zeitschrift für Biologie, 1886, xxii, p. 428.

2 Preparation of fibrin. - With a bundle of smooth rods whip blood as it flows from an artery until the fibrin gathers on the rods. Wash the fibrin in running water until the red corpuscles are removed and the fibrin shows its natural color. Preserve the fibrin in glycerine. 
the suckling calf) to stand twenty-fourhours in 150-200 c.c. $0.1-0.2$ per cent solution of hydrochloric acid. Then neutralize the acid with great care. ${ }^{1}$

Separation of Rennin. - The extract just prepared contains pepsin as well as rennin. The rennin may be separated as follows. The neutralized extract is repeatedly shaken with fresh amounts of magnesium carbonate. The resulting precipitates carry down almost all the pepsin and very little rennin. The filtrate still rapidly coagulates milk, but contains only traces of pepsin. This filtrate is now precipitated with lead acetate, the precipitate is decomposed with very dilute sulphuric acid, and the mixture filtered. To the filtrate, which contains the rennin, is added a solution of stearin soap in water. Thereupon the soap is thrown out of solution and falls, carrying the rennin with it. The soap is then removed by shaking with ether, and the rennin remains. ${ }^{2}$

Precipitation of Casein. - Add 1 c.c. of the neutral extract to 25 c.c. fresh milk at $36-38^{\circ} \mathrm{C}$. (Normal milk is amphoteric. If the reaction be acid, the acid should be very carefully neutralized.)

In a few minutes the milk will separate into

1 Hammarsten : Upsala Läkareforenings Förhandlingar, 1872, viii, pp. 63-86. Abstract by author in Maly's Jahresbericht über die Fortschritte der 'Thierchemie, 1872, ii, pp. 118-125.

2 Hammarsten : Lehrbuch der physiologisehen Chemie, 1895, p. 241. 
curd and whey. The curd is casein together with the fat globules carried down as it precipitates. The whey is a dilute saline solution of milk-albumin, milk sugar, etc.

Test the chemical reaction. The mixture is still neutral. Milk may also be curdled by acid, either added artificially or produced in the milk itself by lactic acid fermentation of milk sugar. The absence of an acid reaction in the above experiment excludes precipitation through acid fermentation of milk sugar. Casein prepared free from milk sugar is also precipitated by rennin. Finally, rennin, extracted by the method given above, does not act upon milk sugar, but rapidly precipitates casein.

Analogy suggests that the specific action of the rennin may be the splitting of casein and that the precipitation may be a secondary process. The following experiments determine this matter.

Experiments of Arthus and Pagès. ${ }^{1}$ - Prepare two solutions, $A$ and $B$.

\begin{tabular}{lr|ll}
\multicolumn{1}{c|}{$A}$. & \multicolumn{2}{|c}{$B}$. \\
Milk & 100 c.c. & Milk & 100 c.c. \\
$\begin{array}{l}\text { Neutral oxalate of } \\
\text { potassium 1\% }\end{array}$ & 5 c.c. & $\begin{array}{l}\text { Neutral oxalate of } \\
\text { potassium 1\% }\end{array}$ & 5 c.c. \\
Renuin 1 to 250 & 4 c.c. & Water & 4 c.c.
\end{tabular}

1 Arthus and Pagès: Archives de physiologie, 1890, p. 334. 
(Rennin, 1 to 250 , is a pastille of Hansen dissolved in 250 c.c. $\mathrm{H}_{2} \mathrm{O}$.)

Keep both mixtures at $38^{\circ}$ C. during forty minutes. 1. Boil 25 c.c. from each solution. Solution $A$ coagulates, while solution $B$ shows no trace of coagulation. Hence the action of rennin has rendered the casein in $A$ coagulable on boiling.

2. To 25 c.c. from each solution add $S$ c.c. of a solution of calcium chloride capable of precipitating exactly, in equal volumes, the solution of potassium oxalate. By this addition any excess of potassium oxalate is removed and the calcium chloride remains in slight excess.

$A$ will coagulate; $B$ will not. Hence the casein in solution $A$ has been so changed by rennin that it is precipitated on the addition of a small quantity of calcium chloride. Solution $A$ may also be precipitated by restoring its original content of calcium chloricle, i.e. by adding 5 c.c. of the above calcium chloride solution, which will exactly combine with the 5 c.c. of potassimm oxalate.

If small quantities of renuin be added to natural milk and equal portions of the milk be tested from time to time by boiling, the amount coagulated will be greater the longer the remnin acts. An amount of calcium chloride too small to produce coagulation in the early stages of 
rennin action is sufficient to produce coagulation when added in the later stages.

Evidently, in the clotting of milk by rennin two separate phenomena must be distinguished: (1) the chemical transformation of casein by rennin, (2) the precipitation of the transformed casein by the calcium chloride. (This salt favors also the splitting of the casein.) Rennin may therefore be classed with pepsin and trypsin.

According to Hammarsten the casein is split into phosphorus-free albumose and phosphorusholding paracasein. Heat is set free. It is the paracasein which precipitates. It is less soluble than casein.

Precipitation of Fibrin by Fibrin Ferment

Buchanan's Experiment. - Press blood clot through a linen cloth. Add the liquid thus obtained to a serous fluid, which does not clot spontaneously, such as ascitic fluid, pleural effusion, hydrocele fluid.

After some hours a firm, translucent clot will form. ${ }^{1}$

Extraction of Fibrin Ferment. Schmidt's Method. - Coagulate one part of serum from the blood

1 Buchanan: London Medical Gazette, 1835, xviii, p. 51; idem, 1845, xxxvi, p. 617. This discovery was first announced in 1831 . 
of ox, dog, or horse, by adding 15-20 parts strong alcohol. After at least fourteen days, filter, dry the moist residue over sulphuric acid, pulverize the dried substance, stir it with water (twice the volume of the serum originally taken) and after allowing sufficient time for solution, filter. The filtrate contains the fibrin ferment. ${ }^{1}$

Gamgee's Method. - Allow freshly prepared fibrin (obtained by washing a blood clot free from corpuscles) to stand three days in 8.0 per cent solution of sodium chloride. Filter. ${ }^{2}$

The filtrate is rich in fibrin ferment.

Extraction of Fibrinogen. - Receive three volumes of blood directly from an artery into one volume of saturated solution of magnesium sulphate, which will prevent the blood from clotting. Separate the corpuscles from the liquid plasma by the centrifugal machine. Add to the plasma an equal volume of saturated solution of sodium chloride. Flakes of fibrinogen will be precipitated. Filter as quickly as possible, for that purpose dividing the liquid among several funnels each with a folded filter paper. Press the filter papers containing the residue between fresh filter paper, in order to remove the adherent

1 Schmilt : Archiv fuir die gesammte Physiologie, 1872, vi, p. 457.

2 Gamgee: Journal of physiology, 1879, ii, p. 151. 
liquid. Tear the filter containing the fibrinogen into small pieces. Dissolve the fibrinogen which sticks to the filter as a tough, elastic mass, in a quantity of 8 per cent sodium chloride solution equal to about one-third the quantity of the magnesium sulphate solution originally taken. Filter off' the fragments of paper. Purify by reprecipitation with an equal volume of saturated solution of sodium chloride. Filter. Dry as before, and add a small quantity of water to the finely divided filter to which the precipitate clings. This water will take a small quantity of salt from the precipitate, and in this dilute saline solution the fibrinogen will dissolve. ${ }^{1}$

Precipitation of Fibrinogen by Fibrin Ferment.Add to the dilute saline solution of fibrinogen a solution containing fibrin ferment.

Fibrin will gradually form.

\section{Ammoniacal Fermentation of Urea by}

\section{UREASE}

1. Place 100 c.c. fresh human urine in each of three clean flasks marked $A, B, C$. To $B$ and $C$ add 1 c.c. of urine that has become ammoniacal upon standing in the atmospheric air. Add also

I Hammarsten : Archiv für die gesammte Physiologie, 1879, xix, p. 563. Also idem, 1880, xxii, p. 431. Hammarsten's first publication was in Nova acta regia societas scientiarum Upsaliensis, 1878, (3), ix. 
to $C \cdot 2$ per cent of a saturated solution of carbolic acid in water. Let $B$ and $C$ stand in a warm place sixteen days.

2. Withdraw 5 c.c. from flask $A$. Note whether the urine is clear or turbid, and whether it effervesces on the addition of a dilute acid. Withdraw 2 c.c. from flask $A$ and determine its percentage of urea by the hypobromite method.

Centrifugalize a portion of the remaining contents of flask $A$. With a microscope examine the sediment for crystals of ammonio-magnesium phosphate and for micro-organisms, especially the micrococcus ureæ, which occurs in long curved chains of round cells about $1.5 \mu$ in diameter.

3. After sixteen days repeat these observations on the urine in flasks $B$ and $C$. Record the results obtained from all three flasks in the table on page 203.

The table shows that the hydrolysis of urea into ammonium carbonate still takes place in urine containing enough carbolic acid to destroy the micro-organisms long known to be the cause of the ammoniacal fermentation. ${ }^{1}$ It is therefore probably due to a ferment, which escapes from the cells after their death.

1 Hoppe-Seyler: Medicinisch-chemische Untersuchungen, Berlin, 1866, p. 570. 
FERMENTATION

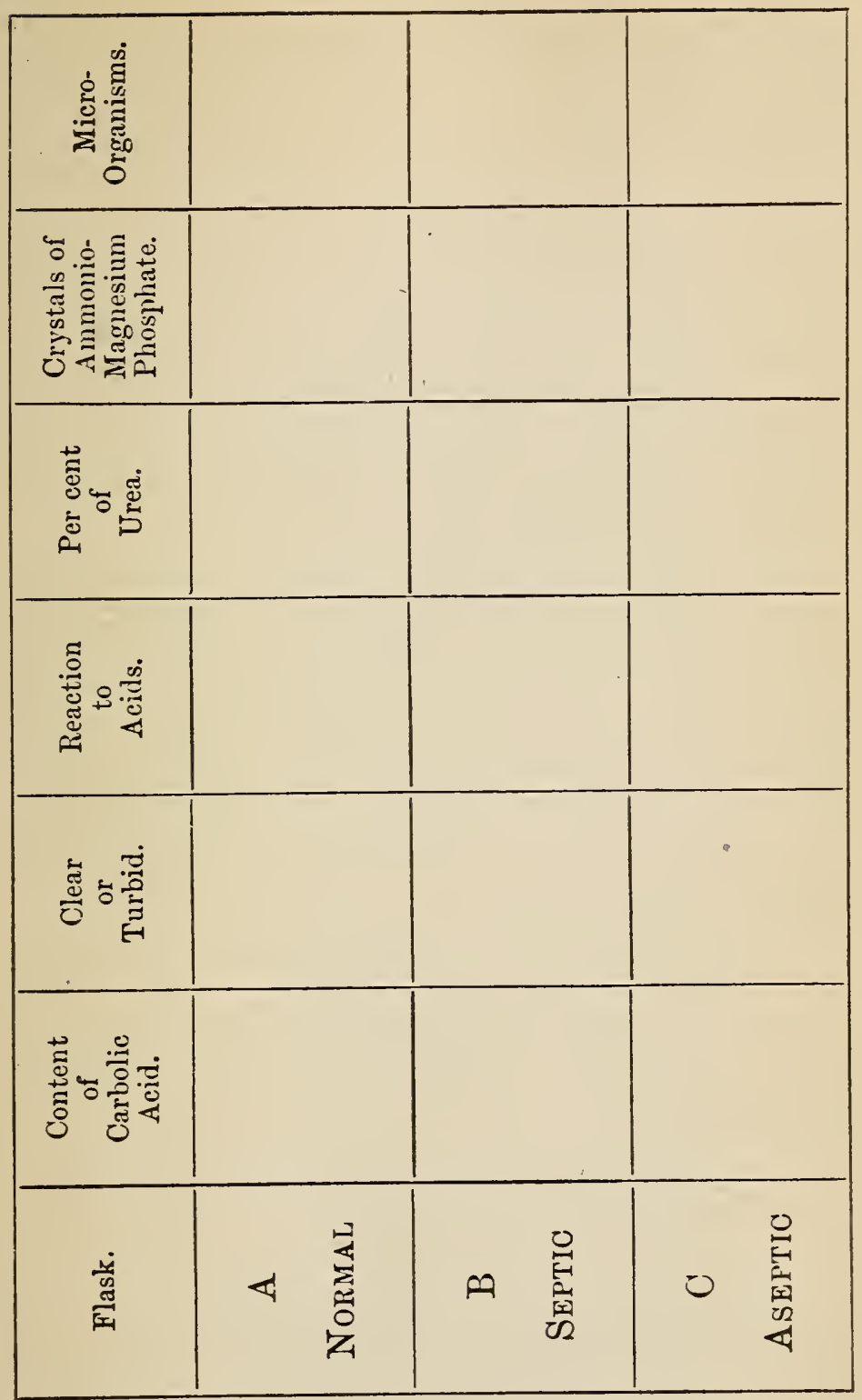


Prior to 1860 ammoniacal decomposition of urine was vaguely classed as a fermentation. In that year Müller ${ }^{1}$ suggested that it might be due to a body like beer-yeast. In 1862 Pasteur $^{2}$ discovered such a yeast, which he called Torula urece. Cohn first classed it with the micrococci. It is aerobic. Miguel finds seven species of bacilli, nine micrococci, and one sarcina, that decompose urea. These obtain their nitrogen ordinarily from proteids, but in the absence of proteids may utilize urea.

Extraction of Urease. - To 10 c.c. of urine undergoing an active ammoniacal fermentation, add 50 c.c. of strong alcohol, and allow the mixture to stand in a well-corked flask. After five days place the precipitate upon a very small filter and wash it with 50 c.c. of fresh alcohol. (Preserve both filtrates for recovery of the alcohol by redistillation.)

1. Add a very small quantity of this precipitate to a neutral 2 per cent solution of urea. Test the reaction. Place the mixture in a water bath at $38^{\circ} \mathrm{C}$.

After a few minutes again test the reaction. It will be strongly alkaline.

1 Müller: Journal für praktische Chemie, 1860, lxxxi, p. 467.

2 Pasteur: Comptes rendus de l'académie des sciences, Paris, 1860, 1, p. 869. See also Van Tieghem, idem, 1864, p. 210. 
After a short time the odor of ammonia will be perceptible. The alcoholic precipitate contains a ferment capable of quickly hydrating urea.

"The alcoholic precipitate from the unfiltered urine consists chiefly of various salts together with the cells of the Torula, hence when treated with water some of the salts are dissolved and pass with the ferment through the filter. If this first aqueous extract be again precipitated with alcohol, a portion of the salts will be again removed, and if this second precipitate be several times redissolved in water and reprecipitated with alcohol, the body with the ferment properties may be ultimately separated - as an amorphous white powder soluble to a clear solution in distilled water and not characterized by any special chemical reactions."

The ferment is not secreted by the cells into the surrounding liquid, but is retained within the cell bodies, for the living cells may be filtered off, and the filtrate will not hydrate the urea. ${ }^{1}$

\section{Splitting and Synthesis of Fats}

Chemistry of Fats and Soaps. - When olive oil is saponified, glycerine appears (Scheele, 1779).

1 Lea : Journal of Physiology, 1885, vi, p. 138. See also Musculus: Comptes rendus de l'académie des sciences, Paris, 1874, lxxviii, p. 132 ; idem, 1876, lxxxii, p. 333 ; Archiv für die gesammte Physiologie, 1876, xii, p. 214. 
It is related to the alcohols (Chevreul, 1813), being a compound ether or ester, a combination of an alcohol with an acid. Commercially glycerine is prepared by exposing neutral fats, such as stearin, to superheated steam, whereby the neutral fat is split into glycerine and fatty acid.

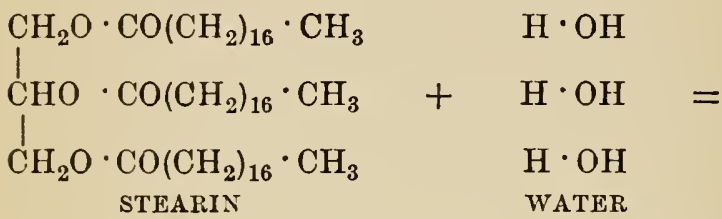

$$
\begin{aligned}
& \mathrm{CH}_{2} \cdot \mathrm{OH} \quad \mathrm{CO} \cdot \mathrm{OH}\left(\mathrm{CH}_{2}\right)_{16} \cdot \mathrm{CH}_{3} \\
& \stackrel{\mathrm{C}}{\mathrm{H}} \cdot \mathrm{OH}+\stackrel{\mathrm{CO}}{\mathrm{l}} \mathrm{OH}\left(\mathrm{CH}_{2}\right)_{16} \cdot \mathrm{CH}_{3} \\
& \stackrel{\mathrm{CH}}{\mathrm{CH}_{2} \cdot \mathrm{OH}} \quad \stackrel{\mathrm{OH}}{ }\left(\mathrm{CH}_{2}\right)_{16} \cdot \mathrm{CH}_{3} \\
& \text { GLYCERINE STEARIC ACID }
\end{aligned}
$$

\begin{tabular}{|c|c|c|}
\hline $\mathrm{CO} \cdot \mathrm{OH}\left(\mathrm{CH}_{2}\right)_{16} \cdot \mathrm{CH}_{3}$ & & $\mathrm{Na} \cdot \mathrm{OH}$ \\
\hline $\mathrm{CO} \cdot \mathrm{OH}\left(\mathrm{CH}_{2}\right)_{16} \cdot \mathrm{CH}_{3}$ & + & $\mathrm{Na} \cdot \mathrm{OH}$ \\
\hline $\begin{array}{c}\mathrm{CO} \cdot \mathrm{OH}\left(\mathrm{CH}_{2}\right)_{16} \cdot \mathrm{CH}_{3} \\
\text { STEARIC ACID }\end{array}$ & & $\begin{array}{c}\mathrm{Na} \cdot \mathrm{OH} \\
\text { SODIUM } \\
\text { HYDROXIDF }\end{array}$ \\
\hline
\end{tabular}

If an alkali be present, it will combine with the fatty acid to form a soap.

$$
\begin{array}{ll}
\mathrm{CO} \cdot \mathrm{ONa}\left(\mathrm{CH}_{2}\right)_{16} \cdot \mathrm{CH}_{3} & \mathrm{H} \cdot \mathrm{OH} \\
\mathrm{CO} \cdot \mathrm{ONa}\left(\mathrm{CH}_{2}\right)_{16} \cdot \mathrm{CH}_{3} & +\quad \mathrm{H} \cdot \mathrm{OH} \\
\mathrm{CO} \cdot \mathrm{ONa}\left(\mathrm{CH}_{2}\right)_{16} \cdot \mathrm{CH}_{3} & \mathrm{H} \cdot \mathrm{OH} \\
\text { SODIUM STEARATE } & \text { WATER }
\end{array}
$$

Splitting of Fats by the Pancreatic Juice. Bernard's Experiment. - Place 2 c.c. neutral olive 
oil in a test-tube and add a small quantity of pancreatic juice (or a piece of fresh pancreas or extract of pancreas). Test the reaction of the mixture. It is alkaline. Note that a white, creamy liquid forms almost immediately. This "emulsion" is composed of a multitude of small fat globules.

Test the reaction again. It gradually becomes acid.

It is evident that under the influence of the pancreatic juice the fatty matter is not simply finely divided and emulsified, but that it has also been modified chemically. ${ }^{1}$

In order to study the splitting of neutral fats by lipase, a ferment found in the pancreatic juice, it is necessary (1) to prepare a perfectly neutral fat, and (2) to recognize the fatty acid as soon as it is set free.

Preparation of Neutral Fat. - Shake commercial olive oil (which always contains fatty acid) for two hours at $95^{\circ} \mathrm{C}$. in a separating funnel with a saturated solution of barium hydroxide. Allow the mixture to stand until the oil separates from the hydroxide. Remove the hydroxide. Filter the oil.

The Emulsion Test for Fatty Acid. Brücke's

1 Bernard : Comptes rendus de l'académie des sciences, Paris, 1849, xxviii, p. 250 . 
Experiment. - 1. Shake 1 c.c. neutral olive oil in a test-tube with 5 c.c. 0.25 per cent sodium carbonate solution.

The oil will be broken up into large globules which will speedily reunite, leaving the liquid clear.

2. Shake 1 c.c. rancid olive oil (containing about 5.5 per cent fatty acid) with 5 c.c. 0.25 per cent sodium carbonate solution.

The mixture becomes instantly milky. The oil is divided into globules of microscopic size. The emulsion is permanent.

3. Shake 1 c.c. neutral olive oil with 5 c.c. water.

The water and oil will not mix.

4. Shake 1 c.c. neutral oil with water containing soap.

The oil will be emulsified. It is probable therefore that soap contributes to the emulsion, perhaps by coating the fine particles of oil with a membrane that prevents their reunion. ${ }^{1}$

Gad's Experiment.-1. Fill a watch glass about $5 \mathrm{~cm}$. in diameter with 0.25 per cent solution of sodium carbonate. With a glass rod carefully place a large drop of rancid olive oil (containing 5.5 per cent fatty acid) upon the surface of the soda solution.

1 Prïcke: Sitzungsherichte der kaiserlichen Akademie der Wissenschaften zu Wien, 1870, lxi, ['1. 613-614. 
The drop will come to rest, and for a moment both the drop and the surrounding liquid remain clear. Very soon, however, the oil is covered with a white layer, and through the soda solution spreads a white cloud which becomes denser and denser until the oil drop, steadily diminishing in size, floats in a milky white liquid.

2. Repeat the experiment, observing the oil drop under a low power of the microscope.

Note the extraordinary motion in the neighborhood of the oil drop, and how the particles of oil are thrown out in strong eddies.

3. Examine the completed emulsion under a higher power of the microscope.

There appear exceedingly small fat drops of very uniform size. The milky fluid is the finest and most uniform enulsion. ${ }^{1}$

Rachford's Experiment. - " Arrange a series of watch glasses containing 0.25 per cent solution sodium carbonate. Place in a test-tube 2 c.c. neutral olive oil and 1 c.c. pancreatic juice (or extract). Shake the tube and allow the juice and oil to separate, then pipette a drop of oil from the surface and place it on the soda solution-in watch glass 1. Again shake the tube and allow the oil and juice to separate, then pipette as before, placing a drop of oil in watch

1 Gad: Archiv für Physiologie, 1878, p. 183. 
glass 2. Again shake and pipette as before, and repeat this process every three or four minutes until the experiment is completed. The beginning of the experiment and the time of each pipetting must be carefully noted. If the pipettings are three minutes apart, then the first drop of oil will have been exposed three minutes to the action of the pancreatic juice, the second drop six minutes, the third nine minutes, and so on. ${ }^{1}$

The gradual increase in fatty acid will be shown by the gradual increase in the amount of the spontaneous emulsion. ${ }^{2}$

It has just been shown that lipase will hydrolyze neutral fats into fatty acid and glycerine. We must now enquire whether this ferment ean effect the synthesis of fats, in other words whether its action is reversible. For this pur-

1 Rachford: Journal of physiology, 1891, xii, p. 81. Rachford used $\frac{1}{3}$ c.c. fresh pancreatic juice obtained by placing a glass tube in the pancreatic duct of the rabbit (see page 80 ).

2 "There is a possible error in this method which had better be spoken of here. It would seem that the alkali of the pancreatic juice would combine with the fatty acids forming soap, and in this way the oil would soon be emulsified in the juice itself and not separate after shaking. This would indeed be a serious ilrawback if it actually ocenrrel, but in truth it does not occur until late in the experiment after we have obtained the information we have sought by the spontaneous emulsion method." (Rachford, loc. cit., 1). 82). 
pose an extract of lipase may be used, first, to split a reutral fat (or glycerol ester) into its constituent fatty acid and alcohol (glycerine is a trihydric alcohol), and second, to form a neutral fat from fatty acid and alcohol.

Extraction of Lipase. From Pancreas. - Remove the pancreas of the pig within thirty minutes after the death of the animal. Dissect off as much of the fat as possible. Reduce the pancreas to a fine pulp in a mortar with coarse wellwashed white sand. Extract the lipase with a little water or glycerine.

From Liver. - Remove the liver of the pig within thirty minutes of the death of the animal. Reduce $50 \mathrm{gms}$. to a fine pulp in a mortar with about 200 c.c. water. Filter. Dilute the watery extract to 500 c.c.

Hydrolysis of Ethyl Butyrate by Lipase. Place in each of two test-tubes, $A$ and $B, 4$ c.c. water, 0.1 c.c. toluene, ${ }^{1}$ and 0.26 c.c. ethyl butyrate. ${ }^{2}$ Cork the tubes tightly. Place them in the water bath for five minutes, to bring them to the temperature of the bath, $40^{\circ} \mathrm{C}$. Add 1 c.c. of

1 Toluene is an antiseptic, which prevents the splitting of the neutral fat by bacteria.

2 Ethyl butyrate hydrolyzes more rapidly than butter fat. It has the further advantage that the amount split by the temperatures employed during the time of the experiment is too small to be measurable. 
the aqueous extract of lipase to each. Boil tube $B$. Place both tubes at $40^{\circ} \mathrm{C}$. for fifteen minutes. Remove them from the bath and plunge them into ice-water (to check further ferment action). Titrate with $\frac{n}{20} \mathrm{KOH}$, using neutral litmus as the indicator. ${ }^{1}$ The initial acidity of the

1 A normal solution contains in each litre one equivalent weight of the active substance, i.e. that mass of the active substance which is equivalent to the atomic weight of a univalent element in the reaction for which the normal solution is to be employed. Equal volumes of different normal solutions are equivalent to each other. Thus, 1 c.c. normal alkali solution requires for neutralization exactly 1 c.c. normal acid, no matter what acid is employed to make the normal solution.

Preparation of Normal Potassium Solution. - The content of $\mathrm{KOH}$ in 1 litre is 56.16 grams. Dissolve 60 gms. purest commercial $\mathrm{KOH}$ (which always contains considerable water) in a graduated cylinder in about 950 c.c. water. Determine the true content of $\mathrm{KOH}$ by titration with a normal oxalic acid solution (prepared by dissolving its equivalent weight $63 \mathrm{gms}$. in 1 litre water) as follows. Thoroughly stir the potassiun hydroxide solution, fill a burette with a portion of the well-mixed solution. Place 10 c.c. normal oxalic acid solution in a beaker and add a few drops of solution of rosolic acid as indicator. Add the alkali from the burette cautionsly until the end point of the reaction is reached, i.e. until the indicator gives a red color which does not quickly disappear. As 10 c.c. of acid solution should exactly nentralize 10 c.c. of alkali solution, provided both were normal, it follows that the quantity of $\mathrm{KOH}$ solution necessary to nentralize is to $10 \mathrm{c}$.c. as the total quantity of the original $\mathrm{KOH}$ solution is to $x$. $x$ will be the number of cubic centimetres to which the $\mathrm{KOH}$ solution must be diluted in order to make it normal. A portion of the normal solution should then be diluted 1:20, and preserved in an air-tight. 
enzyme solution, usually 0.1 to 0.2 c.c. $\frac{n}{20} \mathrm{KOH}$, should be deducted from the cubic centimetres $\mathrm{KOH}$ required to neutralize the fatty acid formed. ${ }^{1}$

Fatty acid will appear in tube $A$, but not in tube $B$, in which the enzyme was destroyed by boiling.

Synthesis of Neutral Fat by Lipase. - 1. Place 5 c.c. $\frac{n}{100}$ butyric acid, 2 c.c. 13 per cent alcohol, 1 c.c. diluted glycerine extract of pig's pancreas (or aqueous extract of liver) in each of two testtubes, $A$ and $B$. Boil the contents of test-tube $B$. Seal both tubes. Keep them thirty-six hours at $48.5^{\circ} \mathrm{C}$.

On opening the tubes, $A$ will give a distinct odor of ethyl butyrate; none will be found in $B$, in which the ferment was destroyed by boiling. ${ }^{2}$

2. Place 5 gms. glycerine, 2 gms. isobutyric acid, 125 gms. water, 1 c.c. neutralized blood serum (or aqueous extract of pig's liver) in each of two

flask. (Compare Müller and Kiliani: Kurzes Lehrbuch der analytischen Chemie, 1900, p. 31 and p. 83).

At $30^{\circ}$ (summer temperature) 0.26 e.c. ethyl butyrate weighs 0.2300 gram. This quantity, if completely hydrolyzed, would require 39.7 c.c. $\frac{n}{20} \mathrm{KOH}$.

1 Kastle and Loevenhart: American chemical journal, 1900, xxiv, pp. 491-525. Also Loevenhart: American journal of physiology, 1902, vi, pp. 331-350.

2 Kastle and Loevenhart: loc. cit., p. 518. 
test-tubes, $A$ and $B$. Boil the contents of tube $B$. Place both at $37^{\circ} \mathrm{C}$. At intervals of half an hour titrate a portion from each tube with $\frac{n}{20} \mathrm{KOH}$ solution. The acidity will diminish in both, but much more rapidly in the tube containing the active ferment.

The acidity is diminished by the combination of the fatty acid with the glycerine to form a neutral fat. ${ }^{1}$

Fats are hydrolyzed to some extent in the stomach, ${ }^{2}$ but stomach lipase is active only in neutral solutions. It is inhibited or destroyed by 0.3 per cent hydrochloric acid. Other ethereal salts besides the fats are hydrolyzed in the intestine, e.g. salol. ${ }^{3}$

The rate of change by lipase increases with the amount of the enzyme, present. ${ }^{4}$

Reversible action is seen in ferments other than lipase, as in the following experiments.

Splitting of Hippuric Acid by Histozyme. - A pig's kidney was perfused four hours with one litre defibrinated pig's blood to which 0.8 gram hippuric acid

1 Hanriot: Comptes rendus de la société de biologie, 1901, p. 70 .

2 Marcet: Proceedings Royal Society, London, 1858, ix, 1. 306. Ogata: Arehiv für Physiologie, 1881, p. 515. Cash: Arehiv für Physiologie, 1880, p. 323.

3 Baas: Zeitschrift fur physiologische Chemie, 1890, xiv, p. 416 .

4 Kastle and Loevenhart: loc. cit., p. 511. 
(sodium salt) had been added. The blood passed through the kidney 9-10 times.

Upon analysis, there appeares 0.087 gram benzoic acid, produced from 0.1276 gram hippuric acid.

Synthesis of Hippuric Acid by Histozyme. - A pig's kidney was perfused three hours with one litre defibrinated pig's blood containing a neutral solution of 0.5 gram benzoic acid and 0.6 gram grlycocoll. The blood passed ten times through the kidney.

Found: $94 \mathrm{mgm}$. hippuric acid. ${ }^{1}$

These actions depend upon a ferment, histozyme, extracted by Schmiedeberc.

Some hypothetical considerations will be of value here. Compounds of carbon may be divided into those in which the carbon atoms are arranged in an open chain, for example ethane, $\mathrm{C}_{2} \mathrm{H}_{6}$,

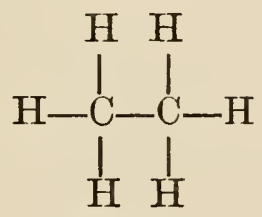

ETHANE

and those in which the chain is closed to form a "carbon ring," for example, benzene, $\mathrm{C}_{6} \mathrm{H}_{6}$, which consists of six carbon atoms, in a closed, ring-shaped chain, the "benzene nucleus," with a hydrogen atom joined to each carbon atom by its fourth affinity (Kekulé, 1865).

${ }^{1}$ Schmiedeberg: Arehiv für experimentelle Pathologie und Pharmakologie, 1881, xiv, pp. 382-383. 


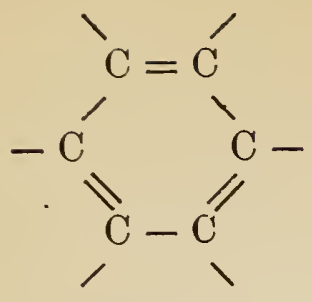

BENZENE NUCLEUS OR RING

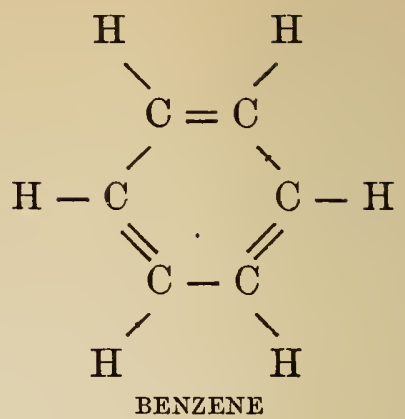

The benzene ring is not easily opened, but derivatives of benzene may be readily obtained by replacing hydrogen atoms. Thus, in aniline or amido-benzene, $\mathrm{C}_{6} \mathrm{H}_{5} \cdot \mathrm{NH}_{2}$, one hydrogen atom is replaced by amide radical; in carbolic acid, or phenol, $\mathrm{C}_{6} \mathrm{H}_{5} . \mathrm{OH}$, by hydroxyl; in toluene or methyl benzene, $\mathrm{C}_{6} \mathrm{H}_{5} . \mathrm{CH}_{3}$, by the radical $\mathrm{CH}_{3}$. The carbon atom in methyl benzene is not a part of the henzene ring, but is chained to the side of the ring. The hydrogen atoms in the side-chain differ in their affinities from those attached to the ring; the hydrogen in the ring may be replaced by groups $\left(e \cdot g \cdot \mathrm{NO}_{2}\right)$ which will not readily replace the hydrogen of the side-chain. This is a matter of special interest in relation to the specific action of poisons, ferments, etc. By substituting hydroxyl for the hydrogen of the side-chain, benzyl alcohol, $\mathrm{C}_{6} \mathrm{H}_{5} \cdot \mathrm{CH}_{2} \cdot \mathrm{OH}$, is formed. By introducing carboxyl, benzoic acid, $\mathrm{C}_{6} \mathrm{H}_{5} \cdot \mathrm{CO} \cdot \mathrm{OH}$, is obtained. It has been shown above that benzoic acid and glycocoll are united in the kidney to form hippuric acid. Glycocoll is amido-acetic acid, $\mathrm{CH}_{2}\left(\mathrm{NH}_{2}\right) \cdot \mathrm{CO} \cdot \mathrm{OH}$. It 
unites with benzoic acid by replacing the hydroxyl in the side-chain, thus forming

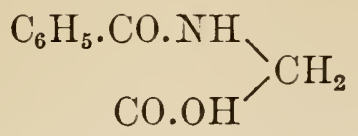

HIPPUIIC ACID

Cinnamic acid, toluene, and other aromatic substances are similarly excreted as hippuric acid when taken internally.

The reversible action of the kidney ferment is important in hastening the establishment of the equilibrium between benzoic acid and glycocoll. If these two bodies pass through the kidney, a certain amount of hippuric acid is formed; if hippuric acid itself passes through the kidney, a certain quantity is hydrolyzed.

Relation of Reversible Action to Absorption of Fat."Pancreatic juice is capable of hydrolyzing all the fat of a fatty meal in the period of pancreatic digestion. In the living intestine the hydrolysis should be complete, inasmuch as the removal of the products of the hydrolysis by absorption prevents the establishment of equilibrium. On the other hand, the products of the hydrolysis in their transition through the epithelial cells come in contact with a lipolytic enzyme, the presence of which in these cells has been demonstrated in the above.

"The lipase now finds itself in contact with only fatty acid and glycerine, and hence in acting catalytically to bring about the chemical equilibrium, it effects 
the synthesis of a fat. This would offer a satisfactory explanation of the presence of fat granules in these cells. As the fatty acid and glycerine diffuse out of the cells through the basement membrane, the fat in these cells would speedily disappear were it not that these substances were constantly being absorbed from the lumen of the intestine. When absorption ceases, however, the fat present is at once hydrolyzed by the lipase present. This hydrolysis is in all probability complete for the reason that the products of the hydrolysis, viz., glycerine and fatty acid, are being constantly removed by diffusion. According to this view, therefore, no fat ever enters or leaves the epithelial cells as such, but as fatty acid and glycerine.

"These two substances then enter the central lacteal, where equilibrium is again established and there is a large production of fat." 1

\section{IMMUNITY}

Ehrlich's Ricin Experiments. ${ }^{2}$ - Powder Albert biscuits weighing 6.75 grams. Add to each cake

1 Kastle and Loevenhart: loc cit., p. 522.

2 Ehrlich: Deutsche medicinische Wochenschrift, 1891, xvii, pp. 976-979.

Ricin is a toxalbumin extracted from the seeds of the castor oil plant. It is poisonous in the slightest traces. Weight for weight it is a billion times more poisonons than colrosive sublimate. Intravenous injection of 0.03 milligram $(0.00003 \mathrm{gram})$ per kilo of body weight is fatal. One gram commercial ricin would kill one and one-half million guinea-pigs. The effect is about one hundred times less when taken by the mouth, yet 
3.2-3.5 c.c. of water containing ricin. The beginning content of ricin should be $0.02 \mathrm{gm}$. ricin for each cake; $0.035 \mathrm{gm}$. is fatal in the course of five or six days. Mix the biscuit powder and ricin solution to a stiff dough, roll the dough into rods, divide them into equal lengths, and dry the portions quickly on a wire sieve. Determine the effect on white mice of successively increasing doses, as follows:

\begin{tabular}{clcc} 
DAY & \multicolumn{1}{c}{ DOSE } & DAY & DOSE \\
1 & $0.002 \mathrm{gm}$. & 9 & 0.02 \\
2 & $\cdots .$. & 10 & 0.03 \\
3 & 0.006 & 11 & 0.04 \\
4 & 0.008 & 12 & 0.05 \\
5 & $\cdots$ & 13 & 0.06 \\
6 & 0.01 & 14 & $\cdots$ \\
7 & 0.0125 & 15 & 0.08 \\
8 & 0.015 & 16 & 0.01
\end{tabular}

On the 17th day inject subcutaneously a fresh mouse with the fatal dose -1 c.c. of a $\frac{1}{200000}$ solution per $20 \mathrm{gm}$. of mouse. At the same time

even thus 0.18 gram will kill a full-grown man. The cause of death is agglutination of red blood corpuscles, and hence multiple thrombosis, especially of the abdominal vessels. Clinically, violent diarrhcea and progressive exhaustion are observed. The toxicity is greatly dependent on species. Guineapigs are far more susceptible than white mice. With white mice the fatal subcutaneous injection is 1 c.c. of a solution containing $\frac{1}{200000}$ ricin per 20 grams of body weight. 
inject the immunized mice with a dose one hundred times as great. ${ }^{1}$

Observe the non-immune and the immune mice for several days and note the results.

Ehrlich continued the above experiment until the immunized mouse received daily $0.5 \mathrm{gm}$. of the ricin by the mouth. Such animals bore safely subcutaneous injections of $\frac{1}{500}$ and even more. The immunity also appeared in that solutions of $0.5-1.0$ per cent applied to the eyes of non-immune mice caused violent panophthalmitis, while immune mice bore easily the application of 10 per cent solutions.

This absolute local immunity was fully established when the general immunity had attained only a middle grade. Normally the subcutaneous injection of $\frac{\overline{4} 01}{400 \overline{0} \sigma}$ ricin solution causes severe local inflammation, but thoroughly immunized animals bear $\frac{1}{1000}$. Quantitative experiments show that the resistance to the poison is not increased during the first four days, and the increase is donbtful on the fifth day, but on the sixth day a relatively high (for example thirteenfold) general immunity is suddenly established. The sudden fall toward normal temperature observed in diseases with a "crisis," such as pneumonia, may depend on the " critical" establishment of immunity.

Immunity is not increased by continued administration of the same dose, day by day. An equilibrium appears to be established.

1 The mice in these experiments must be carefully protected against cold and wetting. 
The immunity once established endures a considerable time; six months and possibly much longer.

Ricin Antitoxine. - Defibrinate the blood of the immunized mice. Divide it into two portions. 1. To one portion add ricin solution in such a ratio that the mixture shall contain $\frac{1}{100000}$, i.e. twice the fatal amount.

Inject a fresh mouse subcutaneously with 1 c.c. of this mixture per 20 grams of weight.

The poison will be borne. It has been neutralized by the serum of the immune animal. This result accords with the discovery of Behring and Kitasato that immunity in diphtheria and tetanus depends on the power of the serum to neutralize the poison.

2. Divide the second portion of the antitoxine blood among six small test-tubes. To the first add a few drops $\frac{1}{10000}$ ricin solution. To the others add amounts increasing in a definite ratio. At first there will be no effect (immunity). As the amount of ricin added is increased, a point will be reached at which agglutination of red corpusles will be produced. This is the neutralization point.

Evidently, there is a definite quantitative chemical relation between the toxine and the antitoxine. 
Theory of Immunity. ${ }^{1}$ - Jenner discovered the protective action of vaccinia against small-pox. The small-pox virus when passed through a susceptible animal becomes attenuated. This weakened poison introduced into the circulation in man protects the individual for long periods against the original disease - it establishes an artificial immunity against smallpox. Schwann found that fermentation and putrefaction arose through the agency of micro-organisms coming from without. Pasteur and Koch demonstrated that the inoculation of animals with pure cultures of certain bacteria produced specific infectious diseases, and that these cultures could be modified at will, either by passing through the animal body, as in Jenner's method, or in artificial culture media. Pasteur produced artificial immunity by using attenuated virus. Behring discovered that the blood-serum of animals immunized against diphtheria contained a substance which would protect other animals against the toxine of diphtheria. So also with tetanus. Ehrlich introduced the quantitative study of toxines and antitoxines by means of test-tube experiments, thereby eliminating the uncertain factor of the animal body. Thus it was shown in experiments on tetanus toxine that the action of antitoxines is accelerated by heat, retarded by cold, dependent on concentration - in short, that it is a chemical action. In the above experiments on ricin, it is shown that the relation

1 Ehrlich: Croonian Lecture, Proceedings of the Royal Society, London, 1901, lxvi, pp. 424-448. 
between toxine and antitoxine is quantitative. These results, obtained by test-tube experiments, have been confirmed by observations on living animals. Thus it was established that a fixed quantity of toxine is neutralized by a fixed quantity of its specitic antitoxine.

Chemical substances affect only those tissues with which they are able to come into chemical contact. They must first reach the tissue. This general law is illustrated by the experiments of Dönitz with tetanus toxine. ${ }^{1}$ When the toxine is injected directly into the circulation and immediately followed by a chemically equivalent amount of antitoxine, the animal is not poisoned; all the toxine circulating in the blood is neutralized. When the same neutralizing dose is injected eight minutes after the toxine, death occurs from tetanus exactly as if no antitoxine had been used. In these eight minutes a lethal quantity of toxine must have left the blood and entered the tissues. This toxine which has entered the tissues may still for a time be withdrawn by injection of the specific antitoxine in quantities much greater than the simple neutralizing dose. The longer the delay, the larger the saving dose. But after a fixed interval, or "period of incubation," no amount of antitoxine, however large, will prevent tetanus. There must, therefore, be present in the brain or cord (the organ principally affected by tetanus toxine) certain atom groups which, like the antitoxine, have a chemical affinity for the toxine. At the close of the period of incuba-

1 Dönitz : Klinisches Jahrbuch, 1900, vii. 
tion the chemical union between these atom groups and the toxine is complete and the antitoxine is shut out. Wassermann ${ }^{1}$ found that when tetanus toxine was mixed with fresh brain or cord substance from the guinea-pig, the toxine united chemically with the nerve centres so that neither the surrounding liquid nor the mixture itself was poisonous when injected into an animal.

The stable benzene ring and the less stable side-chains of the benzene derivatives ${ }^{2}$ suggested to Ehrlich that living cells also consist of a stable centre and less stable side-chains. 'The side-chains enable the cell to form chemical combinations with food stuffs and other bodies that possess atom groups having a chemical affinicy with the atom groups in the side-chains. It is in this way that the toxine is bound to the cell. Experiments have shown that the binding atoms in the toxine molecule are not the poison atoms. If for a portion of fresh toxine there be determined quantitatively (1) the killing power and (2) the amount of antitoxine required to neutralize the toxine, and if the remainder of the toxine be then allowed to stand for a time, it will be found, on again determining the toxic power and the combining power; that the toxic power has diminished, while the combining power remains almost the same. Hence, two separate and independent groups exist. Ehrlich terms the combining atoms the haptophore group, while the poison atoms are the toxophore

1 Wassermann : Berliner klinische Wochenschrift, 1898.

2 See page 216. 
group. The haptophore atom group ( $\ddot{a} \pi \tau \omega$, I cling to) unites with the antitoxine, if there be any present, or with any other atom group for which it has chemical affinity. If this latter atom group be in the side-chain of a living cell, its union with the haptophore atoms of the toxine will necessarily bring the poison atoms of the toxine into intimate chemical relationship with the central atoms of the cell. Poisoning will then take place. If the cells of vital organs have no atom groups with chemical affinity for the haptophore group of a toxine, no union between cell-atom group and haptophore takes place, the toxophore is not brought into intimate contact with the cell, and poisoning does not occur. The animal is naturally immune to this particular toxine. Thus a toxine in sausages is excessively poisonous to man, the monkey, and the rabbit, while even large amounts are not injurious to the dog.

The haptophore group of the toxine acts immediately after injection into the organism, while in most or all toxines the toxophore group becomes active only after a longer or shorter incubation period. During this period the animal may often be saved by placing it in conditions in which the toxophores cannot act. Thus frogs kept at less than $20^{\circ} \mathrm{C}$. are not poisoned by large doses of tetanus toxine, though much smaller doses are fatal at a higher temperature (Morgenroth).

The toxophile atom group of the cell was not predestined to unite with a remotely possible toxine, it has a normal function, probably that of attaching food to the cell. When it enters into its firm and 
enduring union with the haptophore group of a toxine, this normal function is lost. Such a loss acts as a physiological stimulus. ${ }^{1}$ New side-chains are produced by the cell, only to unite with fresh toxine. The production and the loss of side-chains continue until all the toxine in the blood is neutralized. By this time the cell has become habituated to a more than normal production of these special atom groups. The excess is cast off like a secretion and circulates in the blood. These free sille-chains, possessing a special affinity for one specific toxine, constitute the antitoxine of that toxine.

Their continued production after the neutralization of all the toxine protects the animal against fresh toxine, $i$. e. establishes continued immunity.

It has already been stated that by special means the toxophore group of a toxine may be weakened or destroyed while its haptophore group is unchanged. Such altered and non-poisonous toxines are termed toxoids. As their affinity for the sicle-chains of the cells remains unaltered, the toxoids by continuing to unite with the side-chains of the cells may stimulate the production of such side-chains in excess, or, in other words, may assist in making antitoxine and thus establishing immunity.

1 Weigert: Deutsche medicinische Wochenschrift, 1896. 


\section{Haemolytic and Bacteriolytic Ferments}

Bordet's Experiments. ${ }^{1}$ - Inject into the peritoneum of a guinea-pig 10 c.c. defibrinated rabbit blood on five successive days. After two more days bleed the guinea-pig and obtain the serum, by allowing the blood to stand in test-tubes in a cool place until the shrinking clot has pressed out the serum.

1. Mix a drop of serum from a fresh guineapig (one not injected with rabbit blood) with a drop of defibrinated rabbit blood and examine under the microscope. The corpuscles show a very slight agglutination, but are otherwise uninjured. The normal serum of the guinea-pig is almost inactive upon rabbit blood.

2. A. Mix a drop of the serum from the injected guinea-pig with a drop of defibrinated rabbit blood and examine under the microscope. The corpuscles are strongly agghutinated. ${ }^{2}$

B. Mix 0.5 c.c. of the serum with 1.5 c.c. defibrinated rabbit blood.

1 Bordet: Annales de l'Institut Pasteur, 1898, xii, pp. 692694.

2 Agglutinated blood looks granular, especially on gentle shaking; the massed corpuscles sink rapidly ; they will not pass through filter paper. Agglutination of blood corpuscles is similar to the clumping of the typhoid bacillus in the serum of a typhoid-fever patient. 
The corpuscles are agglutinated and their haemoglobin is set free. The mixture becomes red, clear and limpid in two or three minutes. With the microscope nothing can be found but the stroma of the corpuscles, more or less deformed, very transparent and scarcely visible.

The continued presence of blood corpuscles of the rabbit in the blood of the guinea-pig has developed in the latter the power to agglutinate the corpuscles and to set free their haemoglobin. It is thus that the guinea-pig protects itself; it acquires immunity.

3. Heat 1 c.c. of serum to $55^{\circ}$ C. for half an hour. Add 0.5 c.c. of this to 1.5 c.c. defibrinated rabbit blood as in Experiment $2 \mathrm{~B}$.

The serum which was heated to $55^{\circ} \mathrm{C}$. no longer destroys the corpuscles, but still strongly agglutinates them. ${ }^{1}$

Evidently the agglutination of the corpuscles and the setting free of the haemoglobin (termed "laking") are effected by different substances. The agglutinating body resists a temperature that destroys the blood-laking body.

4. To the mixture used in the preceding experiment, add 2 c.c. of fresh serum from a normal

1 A very slow destruetion of the red corpuscles may be observed. This, however, is due to the fresh serum in the 1.5 c.c. defibrinated rabbit blood, as will be evident from Experiment 4. 
guinea-pig (one that has not been injected with rabbit blood).

In a few minutes the mixture becomes limpid and red. The laking power is restored.

Obviously, with the fresh serum was added the unstable body destructive to red corpuscles. Ehrlich and Morgenroth have shown that at low temperatures the stable body unites with the red corpuscles while the unstable body remains in the serum; in this case the haemoglobin is not set free. At higher temperatures the haemoglobin separates and the unstable body is found to have left the serum. It has joined the stable body in the sediment.

Following the side-chain theory already mentioned, Ehrlich and Morgenroth assume that the stable substance has two combining powers; on the one hand it unites with the red corpuscles, on the other with the unstable substance, thus bringing it to the cell which it may then destroy.

Immunity against toxines and foreign red corpuscles are only two of the protective actions of the blood. The injection of cells of the most varied kinds is followed by the production of specific protective bodies $;^{1}$ thus, the injection of

1 Metchnikoff: Annales de l'Institut Pasteur, 1900, xiv, p. 369. 
bacteria causes the formation of bacteriolysines, which destroy the injurious organism.

Many haemolysines and agglutines are found in plants; others, for example, the tetanus bacillus, are bacterial; still others, such as snake venom, are animal secretions.

\section{Oxidizing Ferments}

Schönbein's Experiment. ${ }^{1}-1$. To five c. c. hydrogen peroxide add tinctcre of guiac (freshly prepared by dissolving guiac resin in alcohol) drop by drop until the liquid is milky. Now add from eight to ten drops of a somewhat concentrated extract of malt, prepared in the cold.

The guiac will be oxidized and will turn blue.

2. Repeat the experiment, adding in place of the malt extract from eight to ten drops of blood.

The guiac will be oxidized, as before.

Further Oxidations by Animal Tissues. ${ }^{2}$ 1. Soak strips of bibulous paper in a diluted solution made as follows:

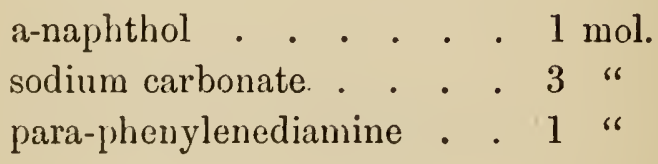

1 Schönbein : Zeitschrift für Biologie, 1868, iv, p. 367.

${ }^{2}$ Spitzer : Archiv fiir die gesammte Physiologie, 1895, lx, vp. $322-323$. 
This solution, left in the atmosphere, oxidizes slowly to indophenol (violet color).

Place a drop of a known oxidizer, e.g. ferricyanide of potash or potassium bichromate, on the saturated paper.

The color will change at once, in consequence of immediate oxidation.

(1) $\mathrm{C}_{6} \mathrm{H}_{4}\left(\mathrm{NH}_{2}\right)_{2}+\mathrm{C}_{10} \mathrm{H}_{7} \mathrm{OH}+\mathrm{O}=$

PARA-PHENYLENEDIAMINE A-NAPHTHOL $\quad \mathrm{NH}<{ }_{\mathrm{C}_{10} \mathrm{H}_{6} \mathrm{OH}}^{\mathrm{C}_{6} \mathrm{H}_{4} \mathrm{NH}_{2}}+\mathrm{H}_{2} \mathrm{O}$

(2)

$$
\mathrm{NH}<{ }_{\mathrm{C}_{10} \mathrm{H}_{6} \mathrm{OH}}^{\mathrm{C}_{6} \mathrm{H}_{4} \mathrm{NH}_{2}}+\mathrm{O}=\underbrace{\mathrm{C}_{\mathrm{C}_{10} \mathrm{H}_{6} \mathrm{O}}^{\mathrm{C}_{6} \mathrm{H}_{4} \mathrm{NH}_{2}}}_{\text {INDOPHENOL }}+\mathrm{H}_{2} \mathrm{O}
$$

Each of the combining molecules has been acted upon by a different oxygen atom; hence the oxygen molecule must have been split.

2. Rub the test paper with finely divided tissue from the liver or any other organ.

Oxidation will occur.

A drop of blood placed on the test paper is soon surrounded by a characteristically colored ring.

Extraction of Nucleo-Proteid from Liver. ${ }^{1}$ Perfuse a fresh liver $(\mathrm{dog})$ with tap water until the washings are no longer colored by haemo-

1 Spitzer: Archiv für die gesammte Physiologie, 1897, lxvii, p. 616 . 
globin. Grind the liver to a pulp and press through several thicknesses of gauze. Add five volumes of distilled water. Allow the mixture to stand twenty-four hours at low temperatures. Remove the opalescent watery extract with a pipette and filter through linen. Demonstrate with the microscope that liver cells are absent from the liquid. Add $\frac{n}{10} \mathrm{HCl}$ drop by drop until there is no further precipitation, and the supernatant fluid is clear. Since the precipitate redissolves in acid, use lacmoid as an indicator. Cease when the lacmoid shows a trace of excess. Decant the precipitate, filter, wash the residue with water.

Oxidation by Nucleo-Proteid. - Place in a widenecked flask 50 c.c. water containing 0.2 gram of the fresh, brown substance and 10 c.c. hydrogen peroxide in a small glass cup. The hydrogen peroxide must be neutralized with from 1 to 1.5 c.c. $\frac{n}{10} \mathrm{NaOH}$. Connect the flask with the lower end of a eudiometer by means of a bent tube. Shake the flask so that the hydrogen peroxide shall come in contact with the tissue. Oxygen is at once set free. Read in the eudiometer the oxygen developed from minute to minute. Spitzer found:

$\begin{array}{lllllllll}\text { After minutes . } & 1 & 2 & 3 & 4 & 5 & 8 & 9 & 16\end{array}$ C.c. $\mathrm{O}_{2}$ developed $19 \begin{array}{llllllll}19 & 28 & 41 & 55 & 69 & 85 & 87 & 95\end{array}$ 
Oxidation about the Nucleus. ${ }^{1}$ - Introduce the oxidizable solution of a-naphthol and para-phenylenediamine (page 230), beneath the cover glass of a fresh preparation of teased thymus or spleen.

"Granules of the intense greenish-blue oxidation product shortly make their appearance within the leucocytes. Their first appearance is typically at the boundary between nucleus and cytoplasm; eventually the latter may become so densely laden as completely to obscure the nucleus. . . The nucleus is the chief agency in the intracellular activation of oxygen. The active or atomic oxygen is in general most abundantly freed at the surface of contact between nucleus and cytoplasm."

Glycolysis in Blood. Bernard's Experiment. ${ }^{2}$ 125 c.c. dog's blood were divided into five equal parts. The sugar in each was estimated as follows :

Sugar

Grams per 1000 .

1. Analysis made at once . . . . 1.07

2. " " " after 10 minutes . 1.01

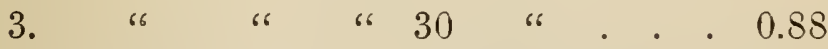

4. " " " " " 5 hours . . 0.44

5. " "

1 Lillie : American journal of physiology, 1902, vii, p. 420.

2 Bernard: Comptes rendus de l'acarlémie des sciences, Paris, 1876, lxxxii, p. 1406. 
Sugar disappears from the blood on standing.

It has been found by Lépine and Barral ${ }^{1}$ that the glycolytic power of the blood increases as the temperature rises to $52.5^{\circ} \mathrm{C}$., which is the optimum. At $54^{\circ}$ the ferment is destroyed.

Oxidation notDependent on Living Cells of Blood. - Place the following solutions at $34-35^{\circ} \mathrm{C}$. for six hours, allowing a stream of air to pass through the liquid. Then estimate the sugar. ${ }^{2}$

\section{A. Calf's blood . . . . . . . . 100 c.c. \\ Water containing 1.14 gram grape sugar 10 c.c. Seegen ${ }^{3}$ recovered 1.000 gram.}

1 Lépine and Barral: Comptes rendus de l'académie des sci. ences, Paris, 1891, exii, p. 146.

2 Test the filtrate by adding a drop of acetic acil and a little ferrocyanide of potassiun.

The absence of a precipitate shows freedom from proteids and ferrie salts. Concentrate filtrate to $150-200$ c.c.

Titration of the Sugar Extract. - Make the volume of the solution such that its probable content of sugar shall lie between 0.0004 and 0.0010 . Causse (Bulletin de la Société chimique de Paris, 1, p. 625 ) recommends that 1750 c.c. of water containing 5 grams of ferrocyanide of potassium be added to each 250 c.c. of Fehling's solution. Boil 10 e.c. of this mixture and ald the sugar solution drop by drop, mitil the blue liquid is decolorized (Arthus: Arehives de physiologie, 1891, p. 425$)$.

${ }^{3}$ Estimation of Sugar in Blood. Extraction of the S'ugar from the Bltort. - T'o 350-400 c.c. boiling water add all at once 50 c.e. blood containing 5 c.c. one per cent acetic acid. Let 


\section{B. Calf's blood . . . . . . . 100 c.c. Water containing 1.14 gram grape sugar 10 c.c. Chloroform . . . . . . . . . 1 c.c. Seegen recovered 0.960 gram.}

The chloroform destroys the cells, but fails to check the oxidation.

Relation of Glycolysis to the Pancreas and the Lymph. ${ }^{1}$ - Remove the pancreas aseptically from an anaesthetized dog which has fasted thirty-six hours. Estimate the sugar in the urine at intervals of a few hour's.

Sugar will be present in large and increasing quantities, ${ }^{2}$ rising even to twenty per cent.

Inject into the jugular vein 15-20 c.c. of lymph from the thoracic duct of a dog fed a few hours before upon one litre of milk.

the mixture boil for a few minutes. Filter throngh a small linen cloth.

Separation of Proteids. - Boil the filtrate. Most of the proteids will separate by coagulation. The remainder, if necessary, may be removed by adding to each 300 c.c. of filtrate, 5 c.c. saturater solution of sodium acetate, and a small quantity of a dilute solution of ferric chloride, neutralizing almost completely with dilnte soda solution, and boiling. The ferric chloride will precipitate as ferrous chloride and will carry down the last traces of proteid substances. Filter. Wash with hoiling water. (Seegen: Centralblatt für Physiologie, 1891, v, p. 824.)

1 Lépine: Comptes rendus de l'académie des sciences, Paris, 1890, cx, p. 742 .

2 Von Mering and Minkowski: Archiv fiir experimentelle Pathologie und Pharmakologie, 1890, xxvi, p. 371. 
The glycosuria will greatly diminish.

After a few hours, the glycosuria will become once more intense, continuing until death. The quantity of sugar in the blood is also greatly increased.

Glycolytic Ferment of Pancreas. ${ }^{1}$ - Remove the pancreas aseptically from a dog immediately after death. Crush it at once in 100 c.c. sterile water containing 0.2 gram sulphuric acid. Allow it to macerate two hours at $38^{\circ} \mathrm{C}$. Neutralize the acid with soda, add 0.5 gram pure glucose, and keep the mixture one hour at $38^{\circ} \mathrm{C}$. Estimate the sugar.

The loss will be from ten to fifty per cent.

When pancreatic extract made without acid is used, the loss of sugar is much less. Probably, therefore, the glycolytic ferment is produced from a zymogen by hiydration.

Malt diastase, or salivary diastase, kept three hours at $38^{\circ}$ in water containing one tenth per cent sulphuric acid loses the power to change starch to sugar, but acquires a glycolytic power.

If the pancreatic juice which flows upon stimulation of the peripheral end of the vagus (Pawlow) is treated with dilute acid, $1: 1000$, the amylolytic power is lost, but glycolytic power is acquired. During the excitation of the nerve - while the juice is flowing - the

1 Lépine: Comptes rendus de l'académie des sciences, Paris, $1895, \operatorname{exx}$, l. 139. 
blood in the pancreatic vein has almost no glycolytic power; after the juice ceases to run, the blood has considerable glycolytic power. Here the external is balanced against the internal secretion of the pancreas.

Oxidative ferments are very widely distributed both in animals and plants. The above experiments show their presence in the blood, pancreas, liver, and lymph. They are present also in the urine.

The stomach contains a ferment that oxidizes lactose to lactic acid. ${ }^{1}$

Urushi, the milky secretion of Rhus vernicifera, dries in the air to a translucent varnish (Japanese lacquer). It contains urushic acid, which does not dry spontaneously, and a ferment, the addition of which to urushic acid causes the latter to dry to lacquer. A sample of fresh juice boiled to stop the action of the ferment on urushic acid contained 15.01 per cent oxygen; lacquer dried in the usual manner contained 20.52 per cent oxygen.

Many oxidations are effected by the tissues without the aid of ferments, so far as is yet known. These belong properly to metabolism, but in passing, it may be noted that while substances exceedingly resistant to oxidation, for example, proteids, are oxidized in the body, other substances very easily oxidizable may be excreted unchanged ; oxalic acid is one of these. ${ }^{2}$

1 Hammarsten : Maly's Jahresbericht der Thierchemie, 1872, ii, p. 118. (Original in Swedish.)

2 Pohl: Archiv für experimentelle Pathologie und Pharmakologie, 1896, xxxvii, p. 413. 
Hoppe-Seyler's Theory. ${ }^{1}$ - Living tissues consist of easily combustible relucing substances, which split the oxygen molecules, taking to themselves one atom of $\mathrm{O}$ and setting the other free in active state to unite with any oxidizable substance present.

Traube's Theory. ${ }^{2}$ - In living protoplasm oxygen is rendered active by an oxidizing ferment, which brings the oxygen to bodies ordinarily oxidizable only by such powerful agents as heat and strong alkalies.

Inorganic bodies, e.g. platinum black, the oxides of copper, silver, mercury, and vanadium, and certain iron salts similarly act as oxygen carriers. Thus

(1) $\mathrm{Pt}+\mathrm{O}_{2}+\mathrm{H}_{2}=\mathrm{PtO}+\mathrm{H}_{2} \mathrm{O}$

(2) $\mathrm{PtO}+\mathrm{H}_{2}=\mathrm{Pt}+\mathrm{H}_{2} \mathrm{O}$

The oxygen carrier reduces $\mathrm{H}_{2} \mathrm{O}_{2}$, takes one atom $\mathrm{O}$ to itself, then gives off this atom in an active or nascent state to oxidize any oxidizable compound present; e.g. guiac. Grape sugar takes O from indigo-blue, producing thereby indigo-white. The indigo-white oxidizes itself to indigo-blue, then gives up another atom of $\mathrm{O}$, and so on.

\section{Alcoholic Fermentation}

The Yeast Plant. - Observe a solution of sugar undergoing alcoholic fermentation. ${ }^{3}$ Note the bubbles of gas, the scum, the sour odor.

I Hoppe-Seyler: Zeitschrift für physiologische Chemie, 1879, ii, p. 1.

2 Traube : Berichte der deutschen chemischen Gesellschaft, 1883, xvi, pp. 123, 1201, and earlier papers in volumes $\mathrm{x}$ and $\mathrm{xv}$.

8 The fermentation is assisted by providing the yeast plant 
Examine some of the mixture under the microscope. Note the multitude of globular or slightly ovoid bodies, the largest about $\frac{1}{10}-\mathrm{mm}$. in diameter. They are motionless. Many have put forth buds. They seem to be plants in active growth. ${ }^{1}$

1. Place 300 c.c. of the nutrient liquid (Experiment 1) in a flask holding 500 c.c. Add a piece of fresh compressed yeast the size of a pea. Place the flask in a temperature of $35^{\circ} \mathrm{C}$.

Note that as fermentation advances the yeast increases in quantity.

2. Place a small piece of fresh compressed yeast in a test-tube. Fill the tube with nutrient liquid and invert it in a dish of similar liquid. The tube may be kept upright by a clamp. Let the mixture stand twenty-four hours in a warm room.

with the salts present in the ash of yeast (Pasteur). A useful substitute is

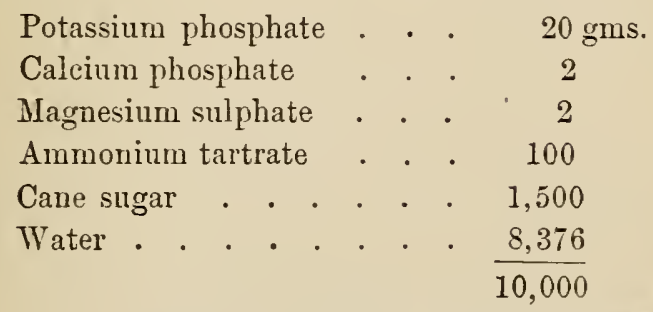

(Practical biology, Huxley and Martin.)

${ }_{1}$ Cagniard-Latour: L'Institut, $183 \overline{5}$, iii, p. 150 ; also Annales de chimie et de physique, 1835, 1xviii, p. 206. The yeast plant was first observed microscopically in beer-yeast by Leeuwenhoek, 1680, but he did not associate fermentation with the growth of the yeast. 
The tube will fill with gas. With a bent pipette introduce about 1 c.c. of a solution of sodium hydroxide (sp. gr. $1.12=11$ per cent). The gas will be absorbed, with formation of sodic carbonate, and the liquid will rise in the tube.

The growth of the yeast plant is accompanied by the production of carbon dioxide.

3. Return to Experiment 1. After the fermenting liquid has ceased to give off gas, place a stopper with a bent tube in the mouth of the flask and distill the contents of the flask in a water bath. Condense the first fifth of the distillate. Saturate this with sodium carbonate. Redistill, and condense.

Test for alcohol by warming the distillate with potassium dichromate and dilute sulphuric acid, whereby the alcohol will be oxidized to aldehyde, with characteristic odor.

Alcohol is present.

The production of alcohol by the yeast is the work of the ferment zymase. ${ }^{1}$ This body is closely bound to the protoplasm of the cell, very easily destroyed, not produced in excess, and not secreted free. Only sugars containing three, six, and nine carbon atoms are attacked. The saccharobioses must be "inverted" before they can be fermented. Thus, cane sugar must

1 Buchner: Berichte der deutschen chemischen Gesellschaft, 1897, xxx, pp. 117, 1110, 2668. 
first he inverted to grape sugar by invertin, ${ }^{\mathbf{1}}$ and malt sugar by maltase. Lactase is present in some yeasts, enabling them to ferment milk sugar. Diastase is also found.

The action of these several ferments becomes clear when the chemical nature of the carbohydrates is recalled.

Chemical Relations of Carbohydrates. - Carbohydrates were furmerly defined to be compounds containing six, or a multiple of six carbon atoms, together with hydrogen and oxygen atoms in the proportion in which they exist in water. The researches of $\mathrm{E}$. Fischer have shown that all aldehydes (bodies which are the first oxidation products of primary alcohols, and which contain the carbonyl group $\mathrm{CO}$ ) and all ketones (bodies which are the first oxidation products of secondary alcohols and which likewise contain the carbonyl group $\mathrm{CO}$ ) contain carbon, hydrogen, and oxygen, there being two atoms of hydrogen to one atom of oxygen, as in water.

The carbohydrates, therefore, no longer occupy an isolated position, but are to be classed with the fats, being methane derivatives in which the carbon atoms are arranged in an open chain; thus, grape sugar is an aliehyde alcohol, and fruit sugar a ketone alcohol.

The carbohychates are divided, according to the size of their molecule, into monosaccharides, disaccharides, and polysaccharides. The monosaccharides (e.g. grape

1 For extraction, see Lea: Journal of physiology, 1885, vi, p. 142. 
sugar) are the first oxidation products of the hexahydric alcohols; the higher carbohylrates are anhydrides of the monosaccharides. Most of the higher carbohydrates cannot be fermented directly, but must first be hyclrolyzed (i.e. take up water). This hydrolysis may be accomplished by the prolonged action of dilute acids at high temperatures, hy the action of water at still higher temperatures, or by specific ferments, e.g. diastase, at the relatively low temperature of the body.

The polysiccharicles, consisting of the starches, the gums (e.g. dextrine or starch gum) and the celluloses (wood fibre) differ greatly from the lower carbohyIrates. The polysaccharides are usually amorphous and are not easily soluble in water.

Carbohydrates. ${ }^{1}$

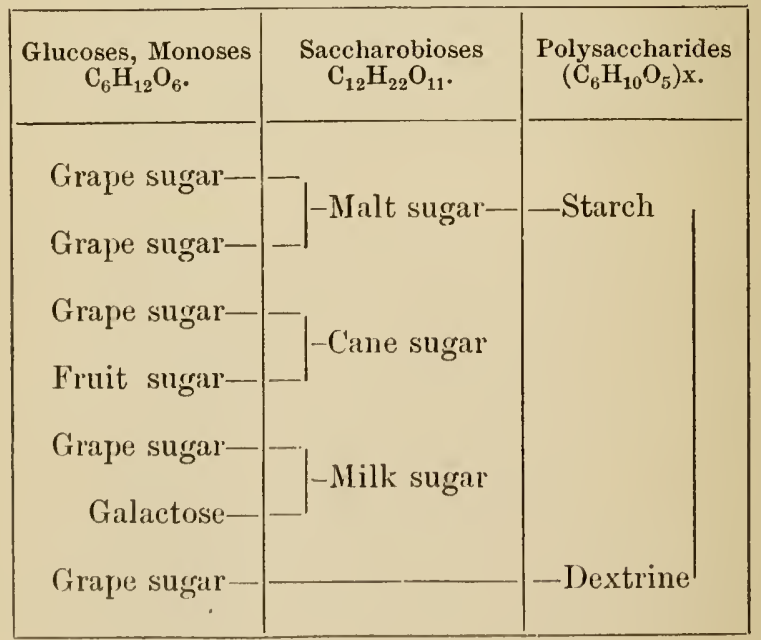

1 Richter's Organic Chemistry, Third Anerican Edition, i, 1. 121 . 
The zymase attacks only those sugars which present a specific stereo-configuration. The position of their atoms in space must fit the position of the atoms of the ferment (the lock and the key). Thus, only the dextro-rotatory forms of the aldehyde sugars (d-glucose, d-mannose, d-galactose) are attacked ; the sugars that rotate the plane of polarized light to the left are not attacked. It is probable that the zymase of different species of yeast presents characteristic differences. It is known that the products formed in the fermentation of sugar by different species of yeasts are to a large degree characteristic. Often these products are injurious. Upon this specific action of ferments rests the work of Hansen, ${ }^{1}$ who tanght the brewers to make pure cultures of the most favorable species of yeast, and thereby raised the brewing industry to the level of an applied science.

\section{Activating Ferments}

Enterokinase. - In 1899, Chepowalnikow, ${ }^{2}$ in Pawlow's laboratory, found that pancreatic juice obtained by Pawlow's ${ }^{3}$ method contained very

1 Hansen: Untersuchungen an der Praxis der GährungsIndustrie, 1895.

2 Chepowalnikow: Thesis (Russian), St. Petersburg, 1899, Paris, 1901.

${ }^{3}$ In Pawlow's method the intestine is resected and the portion of the intestinal wall containing the opening of the pancreatic duct is stitched to the edges of the abdominal wound, where it soon unites; the pancreatic duct then discharges upon the surface of the abdomen, where the juice may be caught by applying a suitable vessel. 
little trypsin and had a correspondingly slight action on proteids. When intestinal juice was added to this pancreatic juice, the pancreatic juice at once became active in proteid digestion. Pawlow called the activating body enterokinase. ${ }^{1}$ In 1902, Delezenne and Frouin ${ }^{2}$ found that pancreatic juice obtained by catheterizing the pancreatic duct contained no trypsin whatever; their procedure prevented any contact between the juice and the intestinal mucous membrane at the orifice of the pancreatic duct. It has been shown that enterokinase is a ferment, secreted in the small intestine, and that it converts trypsinogen contained in pure pancreatic juice into trypsin, the active proteid ferment.

Preparation of Enterohinase. - Scrape lightly with the handle of a scalpel the upper part of the mucous membrane of the small intestine (dog or cat). Digest the scrapings during two days in a closed vessel of water to which a few drops of chloroform have been added to prevent decomposition. Filter through paper, then through a Berkefeldt filter. The resulting solution is perfectly clear, contains a certain amount of coagulable proteid, and will retain its activity

1 PAwLow: The Work of the Digestive Glands, translated by W. H. Thompson, 1902, p. 160.

2 Delezenne and Frouin: Comptes rendus de la société de biologie, Paris, 1902, Yp. 691-693. 
at room temperature for many months. It is rapidly destroyed at $35^{\circ}-40^{\circ} \mathrm{C}$. $^{1}$

Conversion of Trypsinogen to Trypsin by Enterokinase. - Place 5 c.c. of 0.25 per cent solution of sodium carbonate in each of two test-tubes $A$ and $B$, containing gelatine prepared by Fermi's ${ }^{2}$ method. To $\mathrm{A}$ add a few drops of pure pancreatic juice $;^{3}$ to $\mathrm{B}$ add the same quantity of pure pancreatic juice and a few drops of enterokinase. Place at a temperature of $30^{\circ}-35^{\circ} \mathrm{C}$. The pure juice will not act on it, but the juice to which enterokinase was added will dissolve the gelatine. In order to determine accurately the amount of gelatine dissolved, the tubes often must be cooled to $10^{\circ} \mathrm{C}$.

\section{Absorption of Proteids}

Diffusion of Proteids through Dead Membrane. - Bend a cylinder of parchment paper and fasten the ends to a glass rod. Place in the parchment tubes thus formed 25 c.c. of each of the follow-

1 Bayliss and Starlixg: Journal of Physiology, 1903, $\mathrm{xxx}$, p. 80.

2 Fermi and Repetto : C'entralblatt für Bakteriologie und Parasitenkunde, 1902, xxxi, p. 404. Narrow glass tubes, preferably graduated, are tilled one half full of gelatine (5 to 10 per cent) containing one per cent of sodium fluoride.

3 Obtained by catheterizing the pancrentic duct of the rabbit (Rachford's method), Jourual of Physiology, 1891, xii, p. 81. 


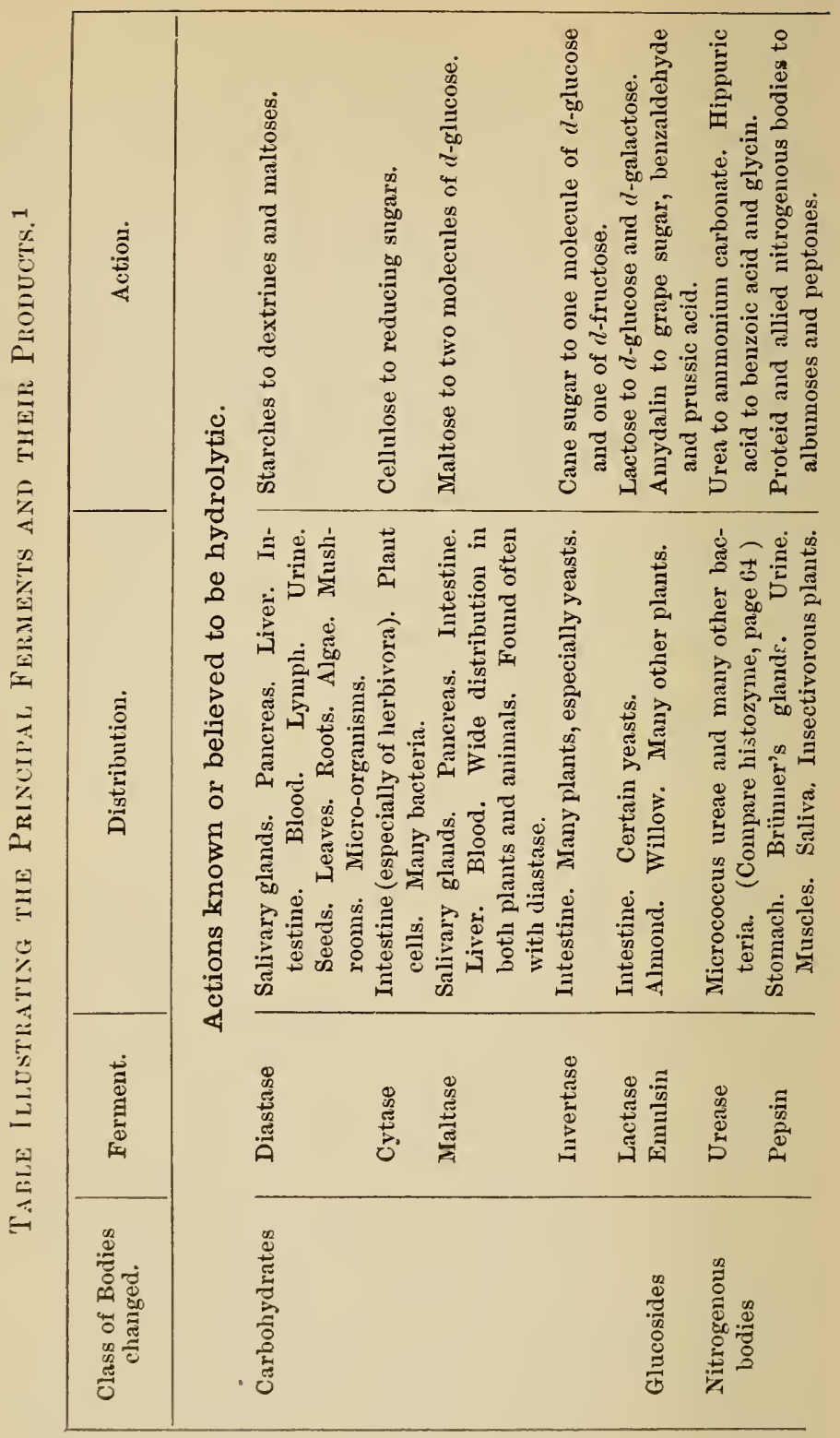




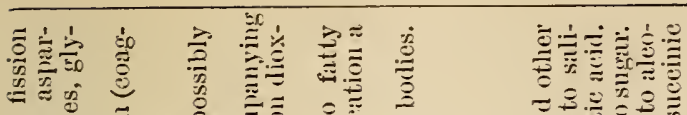

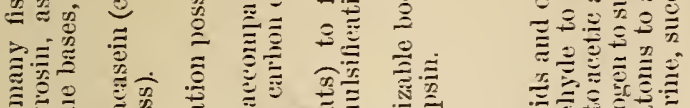

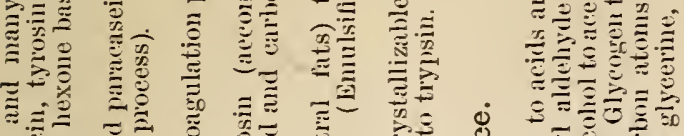

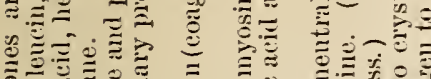

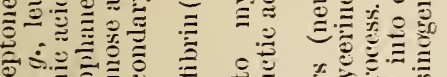

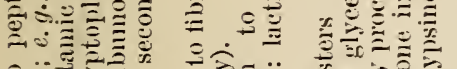
Q 3

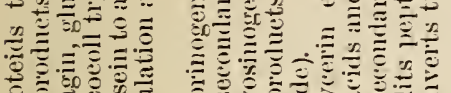

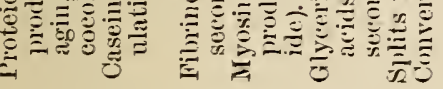

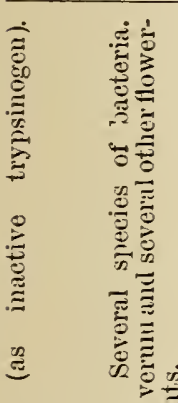

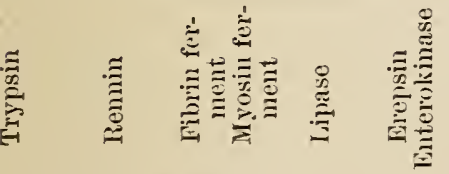
8 일

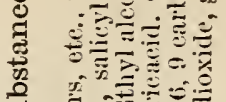
की (2)

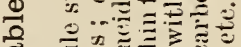
象

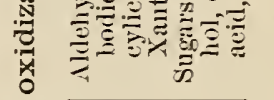

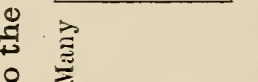

s

窟范

总

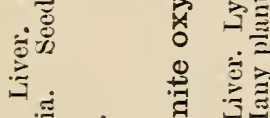

安

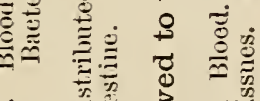

政

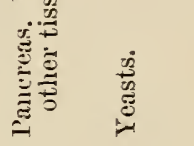

窝

ڤ

ב

है 
ing solutions : ${ }^{1}$ (1) Egg-albumin, (2) Myosin, (3) Alkali-albumin, (4) Peptones. Suspend the eggalbumin and the peptone in vessels containing one litre of normal saline solution ( 0.8 per cent sodium chloride); the alkali-albumin in one litre

1 Preparation of egg-albumin. - Thoroughly mix the whites of twelve or more eggs with saturated solution of magnesium sulphate at $20^{\circ} \mathrm{C}$. Filter from the precipitated globulin. Saturate the filtrate at $20^{\circ} \mathrm{C}$. with sodium sulphate. Filter the precipitated albumin. Press in filter paper. Dissolve in water. Dialyse until the dialysate is free from sulphates. (Starke, quoted by Hammarsten : Lehrbuch der physiologischen Chemie, 1897, p. 372.) Determine the albumin in a measured quantity of the solution, as follows.

Quantitative Estimation of Egg-albumin. - To 25 c.c. of the albuminons liquid add an equal quantity of 4 per cent sodium chloride solution. Neutralize exactly. Boil 5 c.c. of this mixture in a test-tube. To the boiling liquid add one drop of acetic acid from a burette. Filter from the precipitated proteid. Pour the filtrate carefully down the side of a conical glass containing about 5 c.c. concentrated nitric acid, so that the lighter liquid rests on the heavier acid. If the surface of separation remain clear, all the albumin in the 5 c.c. was precipitated by adding to the boiling liquid one drop of acetic acid. If a white ring form, albumin is still present (Heller's test). In this case boil a fresh portion (5 c.c.), add two drops acetic acid, filter, and test the filtrate. Determine in this way how much acetic acid must be added to each 5 c.c. of the albuminous liquid to precipitate all the albumin when the liquid is boiled. Heat 25 c.c. of the remaining portion of the albuminous liquid in a water bath. Add slowly with constant stirring the calculated quantity of acetic acid. Heat ten minutes longer. Filter through weighed paper. Test filtrate once more for albumin. If none be present, wash with water, alcohol, and ether, dry at $40^{\circ}$, weigh, 
of 0.1 per cent sodium carbonate solution; and the myosin in one litre of 5 per cent sodium chloride solution.

\section{After three hours determine the amount of}

subtract the weight of the filter. There remains the weight of the albumin in 25 c.c. of the original solution. ${ }^{1}$

Preparation of Iyosin. - Use muscle containing little blood (calf, rabbit, fowl, frog). Hash the muscle. Wash with water until the washings are free from proteid. Remove excess of wash water by pressure. Mix with enough 15 per cent solution of ammonium chloride to cover the mass. After four hours, filter through cloth and then through paper. The filtrate should be clear, opalescent, somewhat thick. Dialyse in running water. As the nentral salt is removed, the myosin will separate in fine flocks (Danilewsky). Redissolve in 5 per cent sodium chloride solution.

Quantitative Estimation of Myosin. - Dialyse 25 c.c. of the saline solution of myosin until the dialysate is free from salt. Wash the precipitated myosin into a tall narrow beaker. When the niyosin has settled, decant as much of the supernatant liquid as possible. To the remainder add alcohol in such proportion that the mixture shall contain 80 per cent. After coagulation, filter through a weighed filter. Wash with alcohol and ether. Dry at $100^{\circ}$. Weigh. Subtract the weight of the filter.

Preparation of Alkali-albumin. - Warm the whites of twelve or more eggs with 1 per cent sodium hydrate at $40^{\circ} \mathrm{C}$. Filter. Neutralize very cautiously with hydrochloric acid, at first 1 per cent, later 0.1 per cent. The alkali-albumin will be precipitated. Allow to stand several hours. Filter. Boil the filtrate. Filter from the fresh precipitate and add residue to first pre-

1 Literature. - KüHNE: Untersuchungen über das Protoplasma, 1864, p. 2. Danilewsky : Zeitschrift für physiologische Chemie, 1S81, v. p. 158. Halliburton: Joumal of Physiology, 1857, viii, p. 132. KüнNE and Chittexden : Zeitschrift für Biologie, 1Ss9, xxv, 1. 358. 
proteid in each tube and state the per cent that has passed through the membrane.

Diffusion through Living Intestinal Wall. ${ }^{1}$ Through a small opening in the linea alba of a fasting anrsthetized cat ${ }^{2}$ draw out a loop near the middle of the small intestine. Remove the contents by careful stroking. Tie double ligatures $0.5 \mathrm{~cm}$. apart around the intestine at one end of the loop and similar ligatures at a point $30 \mathrm{~cm}$. from the first pair. With a hypodermic

cipitate. ${ }^{1}$ Wash with water. Redissolve in water containing 0.1 per cent sodium hydrate.

Quantitative Estimation of Alkali-albumin. - Neutralize a measured quantity of the solution. Separate the neutralization precipitate upon a weighed filter. Wash with water, alcohol, and ether. Dry and weigh.

Preparation of Peptones. - The separation of peptone from the albumoses ${ }^{2}$ with which it is obtained in the tryptic digestion of proteids is so difficult that it should not be attempted in these experiments. Add commercial peptone, often containing albumoses as an impurity, to a small quantity of boiling neutral distilled water. Filter.

Quantitative Estimation of Peptone. - To 10 c.c. of the liquid add alcohol in such proportion that the mixture shall contain 80 per cent. Filter through weighed filter paper. Dry at $40^{\circ} \mathrm{C}$. Weigh. [This method is not exact, but is to be preferred for the purpose in hand.]

1 VoI'T and BAUER : Zeitschrift für Biologie, 1869, v, p. 562.

2 These and subsequent operations will be done by an instructor assisted by a committee of the class.

1 HAwk and Gies: American Jomrnal of Physiology, 1902, vii, p. 460.

2 KünNe: 'Veitselırift fiir Biologie, 1892, xxix, p. 1. 
needle attached to a burette inject into the loop sufficient egg-albumin solution to distend it slightly. Measure the volume of the solution injected. The content of this solution was found in the course of the experiment on diffusion through dead membranes (page 245).

Replace the loop in the abdomen. At some distance from this loop prepare a control loop with double ligatures in the same way, but leave the control loop empty. Sew up the abdominal wound.

After three hours, kill the animal (best by puncture of the spinal bulb). Remove the loops by cutting between the double ligatures. Rapidly wash the outer surface with water, dry the surface with filter paper, open the loops, measure the volume of the contents, wash the inner surface, add the washings to the contents, and estimate the proteid in a measured portion.

Perform a similar experiment with solutions of (2) myosin, (3) alkali-albumin, and (4) peptone.

Compare the results of absorption of proteids through the living intestinal wall with absorption through dead membranes. It will appear that the living cells of the intestinal wall modify absorption so that it does not follow the law of diffusion through dead membrane.

It is also evident that egg-albumin, myosin, 
alkali-albumin, and peptone may be absorbed unchanged. Indeed, the absorption of alkalialbumin is almost or quite as complete as that of peptone. The conversion of proteids to peptones is advantageous but not essential to absorption.

Absorption Velocity Compared with Diffusion Velocity. ${ }^{1}$ - Prepare a cat as in Experiment 2. Fill one intestinal loop with a measured quantity of 5 per cent dextrose solution, the other with 0.25 per cent solution of sodium sulphate. After one hour kill the animal, measure the liquid remaining in the two loops and estimate its content in dextrose and sodium sulphate respectively. ${ }^{2}$

The dextrose solution will be found to have been largely or completely absorbed, while rela-

1 Rïhmann: Archiv für die gesammte Physiologie, 1887, xii, p. 456.

2 The quantitative estimation of dextrose is described in "Experiments for Students in the Harvard Medical School," third edition, p. 38.

Quantitative Estimation of Sodium Sulphate. - Boil the solution, make the reaction acid with a few drops of hydrochloric acid, add hot solution of barium ehloride in slight excess (until barium sulphate ceases to be precipitated). Boil a few minutes. Wait for the precipitate to settle. Decant the clear liquid through a filter, the ash of which is of known weight. Boil the precipitate in the beaker repeatedly with water. Place the preeipitate on the filter. Wash with boiling water. Dry. Heat to reduess in a weighed crucible. Weigh when eold. (The atomic weights are: barium, 137.4 ; sulphur, 32.06 ; oxygen, 16.) 
tively little of the sodium sulphate will have left the intestine. Yet sodium sulphate is somewhat more diffusible than dextrose. ${ }^{1}$

Assimilable Proteids. - With a catheter remove the urine from the bladder of an anæsthetized female cat, and apply Heller's test for albumin (page 248). Albumin should be absent. Slowly inject into the jugular vein 25 c.c. of solution of alkali-albumin (page 249) at the temperature of the body. Test the urine for albumin twice, at intervals of half an hour.

No albumin will be found. The alkali-albumin has not been removed from the blood by the kidneys.

Non-Assimilable Proteids. - Perform a similar experiment on another cat, injecting solution of egg-albumin instead of alkali-albumin.

Albumin will be found in the urine. Eggalbumin, present in the blood, is at once removed by the kidneys. It cannot be used unless changed ("digested") in the intestine." Albumoses and peptones are also non-assimilable; they produce a dangerous fall in blood-pressure.

1 Compare Hoffuars: Eckhard's Beiträge zur Anatomie und Physiologie, 1860, ii, p. 65.

2 Munk, J., and M. Lewandowsky (Archiv für Physiologie, 1899, Supplement, pp. 73-88) find the non-assimilable proteids of Neumeister (not including albunoses and peptones) may be assimilated if injected very slowly into the blood. 
Alimentary Albuminuria. - Test the urine of a human subject for albumin at half-hour intervals. After the first test let the subject swallow the whites of six raw eggs.

Albumin will probably be found. In many subjects a portion of any unusual quantity of egg-albumin may be absorbed unchanged into the blood, whence it is removed by the kidneys.

Albumose and Peptone not ordinarily Present in the Blood or Urine. - Dissolve as completely as possible ten grams of commercial peptone, which contains albumose as an impurity, in a small quantity of water. Boil. Filter. Measure the filtrate.

Through a small opening in the linea alba of a fasting anæsthetized cat draw out a loop near the middle of the small intestine. Remove the contents by careful stroking. Tie double ligatures $0.5 \mathrm{~cm}$. apart around the intestine at one end of the loop and similar ligatures at a point $30 \mathrm{~cm}$. from the first pair. With a hypodermic needle inject into the loop sufficient peptone solution to distend it slightly. Measure the amount injected. Replace the loop in the abdomen and close the wound. Keep the animal in a cage arranged to collect voided urine.

After two hours, withdraw the urine from the bladder and add it to any that may have been spontaneously voided. 
Bleed the animal from the carotid artery, receiving the blood into an equal volume of saturated solution of ammonium sulphate, to prevent coagulation. Remove the intestinal loop by cutting between the double ligatures. Measure the liquid remaining in the intestine. It will be found that most of the peptone has disappeared. Test the blood and the urine for peptone ${ }^{1}$

1 Recognition of Peptone in Blood. - To the blood already mixed with an equal volume of anmonium sulphate solution add crystals of ammonium sulphate to saturation. Filter from the precipitated proteids. To the clear filtrate apply the biuret test for peptone.

Biuret reaction. - To the saturated ammonium sulphate filtrate add half its volume of saturated solution of potassium hydrate. Shake the dense precipitate. Allow the tube to stand two or three minutes until the heat developed by the chemical action passes off. Add a drop of very dilute solution of cupric sulphate. The fluid, dense white from the precipitated salts, assumes a pale blue color, due to the solution of hydrated cupric oxide in the ammonia generated. The same quantity of saturated potassium hydrate as before is now allowed to flow down the tube, and to form a layer at the bottom. If peptone is present a rose red ring is formed at the junction of the two layers. The contrast of the red ring with the pale blue above it renders the test very delicate (Neumeister's method modified by Shore: Journal of Physiology, 1890, xi, pp. 532-534).

Recognition of Peptone in Urine. - Remove the coloring matter by (1) adding solid lead acetate and filtering from the heavy precipitate; (2) adding to the filtrate ammonium sulphate, and filtering from the copious precipitate of lead sulphate ; (3) saturating the filtrate with crystals of ammonium sulphate and filtering from the additional precipitate. On filtration the 
with the biuret reaction. No peptone will be found. ${ }^{1}$

An examination of the lymph would show that it also contains no peptone. Apparently the peptone absorbed from the intestinal loop is changed in its passage through the intestinal wall. This conclusion is made secure by the experiments of Salvioli, ${ }^{2}$ who removed the jejunum of the dog or rabbit, tied a cannula in the mesenteric artery and vein, and established through these vessels an artificial circulation of defibrinated blood diluted with isotonic saline solution. One gram of peptone was dissolved in 10 c.c. of normal saline solution and placed in the intestine, and the artificial circulation maintained four hours. The peptone disappeared from the intestine, but none could be found in the blood.

Albumose and Peptone changed in their Passage through the Intestinal Wall. - Kill a fairly large ancsthetized rabbit by bleeding. Beat the blood

solution is free from lead, but usually still contains a trace of yellow pigment. Apply the biuret reaction as above. If the urine requires to be concentrated, it is better to evaporate the final ammonium sulphate filtrate, as boiling the urine at first deepens the color. (Nemneister and Shore, loc. cit.)

1 Neumeister: '/eitschrift fiir Biologie, 1888, xxiv, pp. $278-279$.

2 Salvioli : Archiv fiir Physiologie, 1880, Supplemental volumie, p. 112. 
until all the fibrin separates. Filter through gauze into a cylinder holding 100 c.c. To the 30 c.c. defibrinated blood add 30 c.c. sodium chloride solution ( 0.5 per cent) containing 0.6 gram salt-free peptone. The mixture will thus contain 1.0 per cent of peptone. Reserve 5 c.c. of the mixture. Place the rest in a half-litre flask, provided with a stopper pierced by two glass tubes, one reaching to near the bottom of the flask, the other ending just beneath the stopper so that air may be drawn through the blood-peptone solution by an aspirator. Place the flask in a beaker with a heavy iron ring around the neck to prevent the flask being driven upward, fill the beaker with water at $40^{\circ} \mathrm{C}$. and place it in a water bath also at $40^{\circ}$.

Separate the intestine carefully from the mesentery and especially the pancreas. Slit the intestine from the pylorus to the ilio-cæcal valve with scissors, cut it into several pieces, wash it in a large water-bath filled with 0.5 per cent sodium chloride solution at $40^{\circ} \mathrm{C}$. Repeat the washing in a second and a third bath. Cut the intestine into finger-lengths. Collect the pieces on a porcelain sieve, wash them with 1 per cent peptone solution, and then place them in the blood-peptone solution. Draw air through the solution, with every possible care against foam- 
ing. These several operations, beginning with the bleeding of the rabbit, should take not more than fifteen minutes.

After two hours interrupt the experiment, pour the solution through a sieve, stir into the filtrate solid ammonium sulphate to saturation, filter off about 10 c.c., add to the water-clear filtrate an equal volume of absolute sodium hydrate (70 per cent), stir thoroughly with a glass rod, and let the precipitated sodium sulphate settle.

Add to the clear liquid drop by drop 2 per cent cupric sulphate solution. No biuret reaction will be obtained, but the liquid will show at once a pure blue color.

Repeat the test, with the same quantitative relations, upon the reserved 5 c.c. of the bloodpeptone solution: a purple color will be obtained.

Hence the not inconsiderable quantity of peptone in the blood covering the pieces of intestine has disappeared.

Rub the pieces of intestine with sand to a pulp, boil with as little water as possible, saturate with ammonium sulphate, and test as before.

No biuret reaction will be obtained. Hence the peptone which disappeared from the bloodpeptone solution is not stored in the intestine. ${ }^{1}$

1 Nemmeistel: : Zeitschrift für Biologie, 1890, xxvii, pp. 324327. 
Cohnheim ${ }^{1}$ attempted to find in the intestinal wall the peptone which disappears from the intestine without entering the intestinal blood and lymph. His failure led him to the dis-

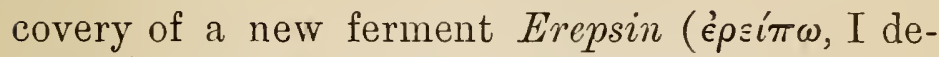
stroy), the action of which is to split peptone into crystallizable substances. This ferment, which is found in many tissues, was isolated by fractional precipitation with ammonium sulphate. Two parts of intestinal extract were mixed with three parts of concentrated ammonium sulphate. The resulting thick precipitate, consisting largely of proteid was dialyzed, and the erepsin found in the dialysate.

The disappearance of peptone from the intestine is probably therefore not to be explained by the assimilation of the peptone or its reconversion into other forms of proteid, but by the splitting of the peptone through the action of the ferment erepsin.

Absorption of Fats, Fat Acids, and Soaps ${ }^{2}$

Absorption of Fat. - 1. Place a few drops of neutral olive oil in the pharynx of a frog that

1 Cohxherm: Zeitschrift für physiologische Chemie, 1901, xxxiii, pp. 451-465.

2 Will: Archiv für die gesammte Physiologie, 1879, $\mathrm{xx}$, pp. 255-262. These experiments are best performed upon summer frogs, $i$. e. not during the normal period of hibernation. 
has fasted at least fourteen days. After about twenty-four hours, remove the intestine and immerse it from thirty to forty minutes in 0.25 per cent osmic acid solution. ${ }^{1}$

Slice the epithelial layer from its base. Tease on a glass slide and examine under the microscope.

Particles of fat stained deep-brown by the osmic acid will be found in the epithelial cells.

In a "control" frog that has fasted fourteen days, show that the intestinal epithelium is free from fat.

2. Open the stomach of a frog the brain and spinal cord of which have been destroyed. Tie a glass cannula in the pylorus. Tie a ligature around the lower end of the intestine. Remove the intestine. Place about 1 c.c. normal saline solution in a test-tube. Hang the intestine in the test-tube by passing the cannula through the cork. Place a little neutral olive oil in the intestine. After about twenty-four hours stain the epithelium with osmic acid and examine as before.

Drops of fat will be found in the cells, but the

1 Preparation of Osmic Acid Solution. - The glass capsule containing a known quantity of osmic acid is placed in a bottle and enough water is then added to make the required solution. The capsule is then broken. [The vapor of osmic acid is very irritating.] 
quantity absorbed will be less than in the living animal.

3. Repeat Experiment 2, using an emulsion of commercial olive oil and 0.25 per cent solution of sodium carbonate.

Absorption will be increased by the giving of the fat in an emulsion.

Absorption of Fat Acids. - Place in the pharynx of a frog a pill of pure palmitic acid made with a few drops of glycerine. After about twenty-four hours examine as before.

Numerous drops of fat will be found in the intestinal epithelium. These globules are not free fat acid absorbed as emulsion, for miscrocopic examination of the contents of the intestine shows no enulsion. Moreover, palmitic acid must be liquid to be enulsified, and as its melting-point is $62^{\circ} \mathrm{C}$. it could not melt in the intestine of a cold-blooded animal at room temperature.

Absorption of Fat Acid as a Soap. ${ }^{\mathrm{l}}$ - Feed a frog with palmitin soap containing a few drops of glycerine. After about twenty-four hours examine the intestine for fat, as before.

1 Preparation of Palmitin Soap. - Dissolve ten grams pure palmitic acid in hot alcohol. Add enough 5 per cent potassium hydrate to combine with the fat acid. Drive off the alcohol by heating on a water-bath. Dilute with water and add a few drops of glycerine. 
Fat globules will be found in the epithelial cells.

\section{LYMPH}

Permeability of Vessel Wall in Inflammation. - 1. In a curarized frog whose brain has been destroyed by pithing spread the mesentery over the glass plate of the mesentery board, and observe the capillary circulation under the microscope. Note the following changes. Dilatation of the arteries, veins, and capillaries, in the order named. With the dilatation an increase in the speed of the blood-stream, most noticeable in the arteries. After half an hour to an hour the acceleration gives place to slowing. All the vessels are now dilated, many capillaries are plainly visible that could hardly be made out in the normal state, the pulsation in the arteries is uncommonly strong down to their smallest branches, yet the circulation is everywhere sluggish. In consequence of the slow blood-stream, the capillaries become crowded with corpuscles, so that they appear redder and more voluminous than normal, yet their cross-section is only slightly increased. In the veins the normally almost clear plasma next the wall fills gradually with lencocytes. The white corpuscles pass through the walls of the veins and capillaries. 
Red corpuscles escape from the capillaries. Hand in hand with the extravasation of corpuscles, there is an increased transudation of lymph. The tissue swells with lymph, which soon exudes upon the free surface of the mesentery, where it clots. The surface is then covered with a fibrinous membrane, crowded with white corpuscles, and containing also some red corpuscles. ${ }^{1}$

2. Place on the frog's tongue a small drop of croton oil mixed with fifty times its volume of olive oil. After thirty seconds wipe off the croton oil. Observe the inflammatory process under the microscope.

3. Place a rubber band around the base of a white rabbit-ear and thus interrupt the venous flow. Hold the tip of the ear in warm water until it has a temperature of about $44^{\circ} \mathrm{C}$. Take the ear from the water and remove the band.

- Note the rosy swelling (œdema with slight extravasation of blood-corpuscles) in the inflamed area. ${ }^{2}$

1 Cohnheim: Allgemeine Pathologie, 1882, i, pp. 237-241.

2 Id.: Loc. cit., pp. 244-245. 


\section{BLOOD}

\section{Specific Gravity}

Drawing the Blood. - Wash the lobe of the ear with a bit of absorbent cotton dipped in clean water. ${ }^{1}$ Rub the lobe dry with another piece of cotton. Pass a three-sided surgical needle through a Bunsen flame. (Do not heat the needle red or the temper will be drawn and the sharpness lost.) Stretch the skin of the lobe between the fingers of the left hand. Make a quick puncture one-eighth inch deep in the edge of the lobe. Press gently to start the flow. The blood must now flow freely. On no account use blood squeezed out.

Determination of Specific Gravity. ${ }^{2}$ - Fill a small beaker half full of a mixture of benzol and chloroform of a specific gravity of about 1059 . Let a drop of the blood fall into this mixture. The drop will remain spherical, for blood does not mix with benzol and chloroform. If the drop sinks, add chloroform drop by drop, meanwhile stirring the mixture with a glass rod, until

1 Subjects who are "bleeders" are not to be used for this observation.

2 Roy: Journal of Physiology, 1884, v, p. ix. HamMerschlac, A.: Wiener klinische Wochenschrift, 1890, iii, 1. 1018. 
the drop neither rises to the surface nor sinks to the bottom but swims with the mixture. If the drop rests upon the surface, add benzol in a similar manner. When the drop neither sinks nor floats, its specific gravity must be that of the benzol-chloroform mixture. Pour the mixture into a glass cylinder, through a piece of linen to hold back the blood-drop, and take the specific gravity of the benzol-chloroform with an areometer. The result is also the specific gravity of the blood.

The values obtained are slightly too low. The error is one unit in the third decimal place.

Determine the specific gravity of the blood under the following conditions. Record the results in the laboratory note-book. Hand to the instructor a copy of your observations written in ink upon a laboratory blank. The material collected by the class will be analyzed statistically by a committee and a report made.

1. The specific gravity of the blood in a healthy man.

2. In the same man half an hour after drinking 750 c.c. of water.

3. In the same man one hour after drinking 750 c.c. of water.

4. In the same man after profuse sweating. Note any feeling of thirst. 


\section{In a healthy woman.}

Hammerschlag found the specific gravity in chlorosis and nephritis diminished as the hæmoglobin diminished. No relation was observed between the appearance of odema and a reduction in the specific gravity.

\section{Counting the Corpuscles}

Counting the Red Corpuscles. - See that the pipettes of the Thoma-Zeiss apparatus are perfectly clean and dry. Open the bottle containing Gower's solution (sodium sulphate, 7.3 grams; acetic acid, 20 c.c.; water, 125 c.c.). Prick the ear as directed on page 264. In a large drop which has collected without pressure put the point of the smaller Thoma-Zeiss pipette ("red counter"). Fill the pipette to the mark 0.5 by careful suction. Should the mark be passed, lower the column to the mark by touching the point of the pipette to filter paper. When the mark is reached, clean the outside of the pipette, dip the end in Gower's diluent solution, and draw the liquic very carefully up to the mark 101. (Should the liquid pass the mark, the pipette must be cleaned and dried and the whole process repeaterl.) Close the ends of the pipette with the fingers, and shake it gently for one 
minute in order to mix the blood thoroughly with the diluent. The blood will now be diluted 200 times its volume.

Remove the rubber tube from the pipette. Blow out the unmixed solution in the capillary tube, between the point and the bulb, and several drops of the mixture in the bulb. Wipe off the end of the pipette. Touch it to the ruled disc. Let a very small drop flow out. Place the cover. glass on the drop. The flattened drop should almost cover the glass. If it spread into the moat, clean the disc and use a second, smaller drop. If Newton's color-rings cannot be seen between the cover-glass and the disc by placing the eyes near the level of the cover-glass, another preparation must be made, with cleaner disc and cover-glass.

Use Leitz No. 5 or Zeiss D objective. Bring the drop into focus and then, using the micrometer screw, find the ruled field.

On the central portion of the disc 1 square millimetre has been ruled into 400 squares, each square having therefore an area of $\frac{1}{400}$ square millimetre. Each 16 small squares are surrounded by double lines, thus forming a "large square." In the Zappert-Ewing slide, the central square of $1 \mathrm{~mm}$. is surrounded by eight other squares of $1 \mathrm{~mm}$. each, and the central ruling is 
extended through the surrounding squares, which are intersected by lines $\frac{1}{4} \mathrm{~mm}$. apart. Count the number of corpuscles, square by square, in 200 small squares. Corpuscles touching the north and south lines of each area are to be counted in, those touching the east and west lines are to be omitted from the count.

Each square has an area of $\frac{1}{40} \overline{0}$ square millimetre. The thickness of the layer of blood, $i$. $e$. the distance from the ruled disc to the coverglass, is $0.1 \mathrm{~mm}$. The volume of the space above each square, therefore, is $\frac{1}{400 \overline{0}}$ cubic millimetre. As the blood is diluted 200 times its volume, and the number of squares counted is 200 , the total number of corpuscles in a cubic millimetre is

$$
\frac{x \times 200 \times 4000}{200}
$$

$x$ being the total number of corpuscles counted. In short, to obtain the number of corpuscles in a cubic millimetre, multiply by 4000 the number counted in 200 squares. Clean the pipette as soon as the counting is done.

Cleaning the Pipette.-Draw clean Gower's solution through the pipette, then alcohol, and finally ether. Dry the pipette by sucking (not blowing) air through it. ${ }^{1}$

1 Do not use alcohol and ether in cleaning the disk. Pipettes left dirty will be eleaned at the student's expense, or, where necessary, a new; one purchased. 
Control Counting.-Count the red corpuscles in a second drop. If the result differ greatly from that of the first count, the corpuscles in a third drop must be counted.

Counting the white Corpuscles. - Have ready a diluting solution of glacial acetic acid (onethird of one per cent). This solution will make the red cells invisible. Obtain a very large drop of blood. By very gentle suction fill the large Thoma-Zeiss pipette to the point 0.5. Keep the pipette nearly horizontal, both in obtaining the drop and in drawing in the diluting solution; the bottle should be tilted. Count the white corpuscles in the entire ruled disc. Repeat with a second drop. Calculate the number of white corpuscles in a cubic millimetre.

\section{Estimation of Haemoglobix}

Oxygen Capacity of the Blood; the Colorimetric Determination of Hæmoglobin. ${ }^{1}$ - Haldane and Smith ${ }^{2}$ have shown that "the coloring power of the blood of different mammals varies in exact

1 Haldaxe : Journal of Physiology, 1901, xxvi, pp. 497504. This experiment should be substituted for that giren in "Experiments for Students in the Harvard Medical School," third edition, pp. 100, 101.

2 Haldaxe and Surth : Journal of Physiology, 1900, xxv, pp. 331-343. 
proportion to its oxygen capacity. The latter can be easily and accurately determined by means of the ferricyanide method. ${ }^{1}$ Thus blood - of a certain oxygen capacity has also a certain coloring power; and it is possible to standardize the coloring power in terms of the oxygen capacity. We can therefore make the unit of volume the basis of our definition of the unit of coloring power employed in hemoglobin estimations. Since, however, oxy-hiemoglobin is not stable, I have adopted as a standard a dilute solution of blood of known oxygen capacity saturated with coal gas. ${ }^{2}$ This solution is sealed up in a narrow test-tube after all the contained air has been displaced by coal gas, and when thus completely sealed is permanent."

The standard solution for the himoglobinometer is a one per cent solution, saturated with coal gas, of ox or sheep's blood of the average oxygen capacity of the blood of normal adult males, found to be 18.5 per cent. If it be borne in mind that 100 per cent on the hæmoglobinoneter scale corresponds to an oxygen capacity

1 In this method the oxygen is displaced from laked blood by ferricyanide of potassium, and the resultant gas measured. HALDANE: Journal of Physiology, 1900, xxv, pp. 295-302.

2 Coal gas eontains earbon monoxide as an impurity, and thus converts the oxy-hæmoglobin to CO-hæmoglobin. 
of 18.5 per cent, it is of course easy to express the results in terms of oxygen capacity. The exact percentage of hæmogiobin corresponding to 18.5 per cent oxygen capacity is still uncertain. According to Huifner's latest results it would be 13.8 per cent.

In using the hæmoglobinometer, place 15-20 c.c. water in the graduated tube, for dilution of the blood. Draw $20 \mathrm{cb} . \mathrm{mm}$. of blood into the pipette, with the necessary precautions. ${ }^{1}$ Gently blow the blood out of the pipette on to the surface of the water in the graduated tube. Before mixing the blood with the water introduce into the free part of the tube a narrow glass tube connected with the gas-tap, turn on the gas, and push the gas-tube down to near the level of the water, so that the air may be instantly displaced from the tube. Avoid any loss of liquid. If the upper part of the tube, or the liquid itself, is warmed by the fingers while the solution is being mixed or saturated with carbon monoxide, a little liquid is apt to spurt out. This can be avoided by holding the tube in a cloth. Withdraw the gas-tube while the gas is still flowing. Close the top of the graduated tube with the finger and invert the tube about a dozen times,

${ }^{1}$ See page 264 ; remember not to use blood squeezed from the ear. 
so that the hæmoglobin is thoroughly saturated with carbon monoxide and the full pink tint of the CO-hæmoglobin appears. Then add water drop by drop from a pipette until the tint in the graduated tube equals that in the standard tube. In comparing the tints of the two tubes, it is best to hold them up against the light from the sky. The precaution must always be taken of repeatedly transposing the tubes from side to side during the observations: otherwise very considerable error may arise. The percentage is read off on the tube after half a minute has been allowed for the liquid to run down. Another drop is now added, and if necessary another, until the tints again appear unequal. Usually the tints will appear equal for two or possibly three additions. The mean of the readings which gave equality is taken as the correct result. The results in successive experiments with the same blood should agree within one per cent of the inean.

The average percentage of hæmoglobin in the blood of women is 11 per cent, and in the blood of children 13 per cent below that of adult men. In calculating the proportion of hrmoglobin in the blood of women and children as percentages of the average normal proportion, it is evidently necessary to add about one-eighth for women 
and one-seventh for children to the percentage found by the hæmoglobinometer, with the standard solution described above.

\section{Hæmorrhage and Regeneration}

Determine the specific gravity, number of red and white corpuscles per millimetre, and percentage of hæmoglobin in the same animal under the following conditions: Normal; two hours after a profuse hæmorrhage; one day, three days, and five days after the hæmorrhage. Plot all three curves upon one co-ordinate system.

\section{Physical Aspects of Coagulation}

Physical Action of Salts in the Coagulation of Colloidal Mixtures. - 1. Boil egg-albumin diluted with about eight volumes of water. The colloid will not coagulate. Add crystals of magnesium sulphate gradually. Coagulation will take place. ${ }^{1}$

2. Dip a thin thread of silk in 2 per cent solution of calcium chloride and lay the thread upon a glass slide beneath a cover-glass. Allow boiled solution of egg-white $(1: 8)$ to run under the cover-glass. Examine the process of coagulation under a magnification of about 500 diam-

1 Haycraft and Duggan: British Medical Journal, 1890, p. 167 . 
eters. The fluid at first is free from visible particles. Near the silk thread appears a fine cloud, the particles in which grow in size until they form spherules having a maximum diameter of 0.75 to $1 \mu$. They are now seen to be arranged in patterns forming an open net with regular polygonal meshes, having diagonals as long as $6 \mu$. The threads of the net are formed of contiguous sphierules. This stage, however, is not one of equilibrium — the net shrinks, the meshes become smaller, and the spherules apparently shift their points of attachment until, in place of being bounded by threads composed of several spherules, the image has the appearance of the typical fine net with spherules at the nodal points joined by tiny threads. Whether these joining-threads or bars have a real existence, or whether they are purely optical and the spherules actually touch one another, it is impossible to say at present. When the particles are large enough to be clearly visible with a magnification of 500 diameters they do not show Brownian movement - in other words they are probably already in some way linked to one another.

The following explanation of these phenomena may be given. On boiling the egg-allumin, the heat chemically alters the dissolved proteid and produces a suspension of particles having an 
average diameter commensurable with the mean wave-length of light. ${ }^{1}$ Under the influence of electrolytes (the salt solution) the particles aggregate to larger and larger masses. When these molecular aggregates attain a certain size the fluid condition is no longer possible; this would follow immediately from Graham's observation that actual coagulation is preceded by a continuous increase in the viscosity of the liquid. The following conditions determine this generic action of salts as coagulants, as distinguished from any specific chemical action. 1. The point at which coagulation appears is determined by the concentration of the solid in the colloidal mixture, and the temperature, molecular concentration (gram-molecules per litre), and nature of the electrolytes present. 2. The concentration necessary for coagulation is lowered by a rise of temperature, or by an electrolyte. 3. The coagulative energy of electrolytes as measured by the number of gram-equivalents per litre necessary to produce coagulation is determined almost solely by the nature of the metal of the salt; and amoug the metals themselves it is determined by the valency of the metal. ${ }^{2}$

1 Picton and Linder: Transactions of the Chemical Society, 1895, 1xvii, p. 63.

2 HARDY : Journal of Physiology, 1899, xxiv, pp. 181-183. 
Physical Changes in Coagulation. - 1. Clotting of Plasma. - Wet a small filter with cane-sugar solution ( 0.5 per cent). Cut a frog's ventricle across near the base so that 0.5 c.c. blood shall fall into a beaker containing an equal quantity of canesugar solution. Pour the mixture on the filter. Receive the filtrate on a watch-glass. Note the physical changes in this filtrate.

2. Fibrin Threads. - Place a blood-drop under a cover-glass. With the microscope observe the appearance of fibrin threads.

3. Receive 1 c.c. blood into 0.5 c.c. saturated solution $\mathrm{MgSO}_{4}$. Note (1) absence of clotting, and (2) its appearance after dilution.

4. Receive 0.5 c.c. blood in a watch-glass. Let it stand twenty-four hours. Note physical changes during the first ten minutes and at end of period.

\section{SECRETION}

Speed of Absorption and Secretion. - Place 5 c.c. of thin starch paste and 2 c.c. concentrated nitric acid in each of ten test-tubes and mark them $2,4,6,8,10,12,14,16,18$, and 20 minutes. Let one of each pair of students swallow a gelatine capsule containing ten grains of potassium iodide. Immediately rinse the sub-

1 The subject should have had a small, early breakfast. 
ject's mouth until the wash water gives no blue color (iodide of starch) on the addition of potassium iodide and concentrated nitric acid (to set free the iodine). Let the subject chew a small piece of clean black rubber-tubing to increase the secretion of saliva. At intervals of two minutes, beginning with the swallowing of the potassium iodide, empty the mouth into the corresponding test-tube, at once rinse the mouth with water, and begin a fresh collection. ${ }^{1}$

Note the moment at which the drug appears in the saliva.

\section{RESPIRATION}

\section{Chemis'try of Respiration}

Estimation of Oxygen, Carbon Dioxide, and water. ${ }^{2}$ - Weigh bottles 3,4 , and 5 (4 and 5

1 If the saliva secreted during two minutes cannot be held in the month with comfort and without loss by swallowing, the mouth may be emptied into a freshly washed porcelain dish, from which the saliva should be poured into the proper testtube at the end of each two-minute period.

2 Apparatus. - Two aspirator bottles, with box. A wooden tray, containing a jar for the gninea-pig, and six bottles, viz. : Nos. 1 and 4, filled with soda-lime, to absorb carbonic acid; Nos. 2, 3, and 5, filled with pumice stone soaked in sulphuric acid, to absorb moisture; No. 6, a Miuller valve, to prevent air being forced back through the series of bottles by a wrong coupling of the aspirator tubes. 
together). Place the guinea-pig in the jar and weigh. During one hour draw air through bottles 1 to 6 by placing an aspirator bottle on its box and allowing the water to flow from this bottle to the one remaining on the desk. The rubber connecting tube must be changed when the aspirator bottles are changed. After one hour weigh bottle 3 , and bottles 4 and 5 .

Tabulate results as follows:

grams

$\underset{6}{\text { Weight }} \underset{\text { a jar and guinea-pig at beginning }}{\text { a }}$

Loss

Wt. of bottle 3 (sulph. acid) at beginning "6

$$
\text { " " end . . }
$$

Gain (= water absorbed) . . .

Weight of bottles 4 and 5 at beginning . “ " " end . . .

Gain (= carbon dioxide absorbed)

Total water and carbon dioxide absorbed Loss in weight of jar and guinea-pig .

Difference (= oxygen absorbed) .

Respiratory quotient

\section{Metabolism}

Effect of Muscular Exercise on the Oxygen, Carbon Dioxide, and Water of the Respired Air. liepeat the estimation of oxygen, carbon dioxide, 
and water in the respired air (p. 277), slowly turning the guinea-pig jar from side to side, so that the animal shall be kept in gentle motion during an hour.

The excretion of carbon dioxide is increased by muscular exercise.

Individual Level of Proteid Metabolism. - Each group of eight students will select two subjects for experiment. They should be thin men in good health. Let each subject collect the twentyfour hours' urine in a thoroughly clean bottle of about 2000 c.c. capacity. Measure the quantity. Determine in a measured portion of the total mixed urine the quantity of urea (hypobrobromite method). Calculate the nitrogen in the urea. Add 2.5 grams for the nitrogen excreted in the fæces, sweat, and as uric acid in the urine.

Repeat these determinations for three days, the subject maintaining his usual diet and mode of life.

The excretion of nitrogen will probably be found to be fairly uniform in each individual though the different nutritive habits of different individuals may cause them to be on different proteid planes, characterized by high, medium, or low nitrogen excretion.

Nitrogenous Equilibrium. - When the daily 
excretion of nitrogen has been found to be fairly uniform, place the subject upon a simple diet of eggs, bread, milk, and butter, containing as much nitrogen as he excretes. ${ }^{1}$

The diet may be chosen from the following table. $^{2}$

The relative proportion of proteid, fat, and carbohydrate per day should be about as follows:

$$
\begin{aligned}
& \text { Proteid . . . . } 100 \text { grams } \\
& \text { Fat . . . . } \frac{100 \text { grams }}{\text { Carbohydrate . . }} \frac{250 \text { grams }}{450 \text { grams }}
\end{aligned}
$$

Repeat the determination of urea in the urine during four more days. ${ }^{3}$ Calculate the nitrogen

1 Owing to the variation in the nitrogen content of meats, they should be omitted.

Physiological heat vulues :-

$$
\begin{aligned}
& 1 \text { gram proteid }=4000 \text { small calories } \\
& 1 \text { " fat }=9423 \text { " " carbohydrate }=4182 \text { " " }
\end{aligned}
$$

Proteids contain about 16 per cent nitrogen. Hence to obtain the amount of metabolized proteid from the nitrogen in the urine multiply the latter by 6.25 .

In one pound there are 453.6 grams.

2 Coffee and tea contain so little nitrogen that they may be added to the diet in small anounts to suit the individual taste.

3 The twenty-four hours should begin in the moning immediately after passing the mine exereted during the night. 


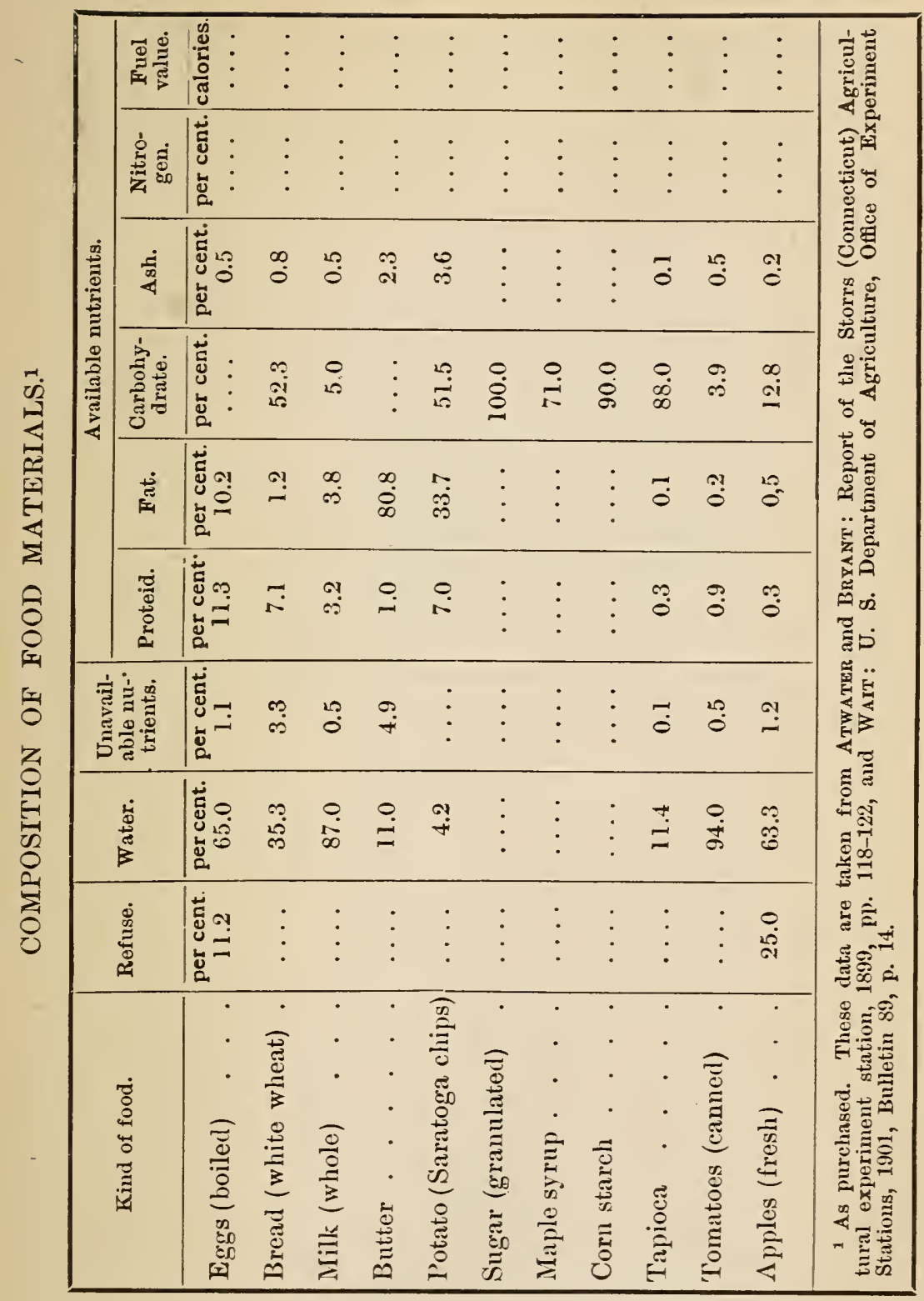


excreted in urea, adding 2.5 grams for the nitrogen of the freces, sweat, uric acid, etc.

It will probably be found that the nitrogen excreted equals that ingested (nitrogenous equilibrium).

Effect of Muscular Exercise on Proteid Metabolism. - On the third day of the preceding experiment, the subject should take an unusual amount of measurable exercise. Hill-climbing is a suitable form. The protocol of the experiment must contain an approximate estimate of the work done in foot pounds.

It will be found that muscular work, unless pushed to great fatigue, does not increase proteid metabolism, as measured by the nitrogen excreted. 


\section{PART III}

THE OUTGO OF ENERGY 



\section{P A R T III \\ THE OUTGO OF ENERGY \\ I ANimal Heat}

Regional Temperature. - Record the temperature taken with a clinical thermometer placed under the tongue, in the axilla, and in the rectum.

Effect of Hot and Cold Drinks on the Temperature of the Mouth. - Record the temperature taken under the tongue soon after drinking (1) , cold water, (2) warm water.

Hourly Variation. - Record the temperature taken under the tongue every two hours through the day and plot the results on clinical temperature paper.

Reaction of Cold and Warm Blooded Animals to Changes in the External Temperature. - 1. Record the temperature in the gullet of a frog placed in water of varying temperature. 2. Record the (rectal) temperature of a guinea pig in a bath at $40^{\circ}$, gradually cooled to $15^{\circ} \mathrm{C}$.

Chemical Action the Source of Animal Heat. Calculate the heat values observed by Rubner ${ }^{1}$

1 RUBNer : Zeitschrift für Biologie, 1894, xxx, p. 134. 
in a dog weighing $11.8 \mathrm{~kg}$., receiving 580 gms. flesh daily. ${ }^{1}$

\begin{tabular}{|c|c|c|c|}
\hline & Total & Fat & Calories \\
\hline 1890. & excreted. & $\mathrm{C}$ & from Proteid; from Fat. \\
\hline
\end{tabular}

$\begin{array}{rrr}\text { April } 11 & 18.75 & 14.6 \\ 12 & 17.39 & 18.2 \\ 13 & 19.43 & 13.7 \\ 14 & 18.35 & 17.5 \\ 15 & 18.57 & 17.5 \\ 16 & 18.50 & 16.8 \\ 17 & 18.31 & 17.1\end{array}$

Compare above results with those actually obtained by Rubner when the dog was placed in the calorimeter.

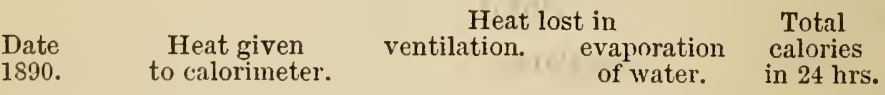

$\begin{array}{rrrrr}\text { April } 12 & 469.4 & 44.8 & 165.1 & 679.4 \\ 13 & 483.0 & 56.4 & 148.6 & 687.9 \\ 14 & 455.3 & 32.9 & 178.0 & 666.1 \\ 15 & 485.1 & 34.4 & 179.1 & 698.7 \\ 16 & 447.9 & 34.7 & 199.3 & 681.8 \\ 17 & 465.9 & 34.1 & 174.2 & 674.2 \\ 18 & 456.9 & 35.1 & 187.3 & \mathbf{6 7 9 . 4}\end{array}$

$11 \mathrm{gm}$. proteid $=4.1 \mathrm{cal}$. (Rubner used 4.0 cal.)

$$
1 \text { " fat }=9.3 \text { " ( " " } 9.423 \text { " ) }
$$

To find the fat from the carbon inultiply the carbon by 1.3 (fat contains 76.5 per cent of carbon). 


\section{II}

THE ELECTROMOTIVE PHENOMENA OF MUSCLE AND NERVE

The stored energy of muscle is set free in molecular movement, - heat, chemical action, and electricity, - and in mechanical work, the change in form. It will be convenient to consider the electromotive phenomena first.

\section{The Denarcation Current of Muscle}

Demarcation Current of Muscle. - 1. Mount two non-polarizable electrodes. Connect them to the capillary electrometer through a shortcircuiting key. Remove a sartorius muscle. Cut off each end with a sharp knife by a clean cut at right angles to the fibres. Observe that the muscle is thereby converted into a "muscle prism." It possesses two artificial cross-sections. at each of which the muscle has been injured, and is, in fact, dying, and an uninjured natural longitudinal surface. Place the muscle across the electrodes so that the cross-section rests on one electrode and the middle of the longitudinal surface rests on the other. Bring the meniscus of the capillary into the field. Note its position on the micrometer scale. Open the key. 
The meniscus will be displaced in the direction indicating a higher potential at the middle or "equator" of the longitudinal surface than at the cross-section. Note the divisions of the scale, traversed by the meniscus - the displacement is proportional to the difference of potential.

2. Move the electrode on the longitudinal sur.face a few millimetres towards the cross-section. Determine the difference of potential here. It will be less than before. Measure the potential in similar manner at intervals of $5 \mathrm{~mm}$. between this point and the cross-section. On co-ordinate paper set down on the abscissa the number of millimetres from equator to cross-section. Set down as ordinates the number of divisions of the micrometer scale traversed by the meniscus when the electrode on the longitudinal surface is placed successively on the equator, and at intervals of 5 $\mathrm{mm}$. between equator and cross-section. Draw the curve uniting the summits of the ordinates.

As the cross-section is approached, the curve of potential will fall more and more rapidly. The centre of the cross-section is negative towards the outer parts of the section. Points on the equator, or equidistant from it, have the same potential. Points on the longitudinal surface at different distances from the equator, and on the cross-section at different distances from the 
centre of the section, show a slight difference of potential.

Prove these several statements.

Oblique Section. - When the artificial crosssection is oblique to the long axis of the muscle, the maximum difference of potential is no longer at the equator and the centre of the cross-section. The most positive point is on the longitudinal surface near the obtuse angle made by the oblique section, and the most negative point is on the cross-section near the acute angle. The structure of certain muscles, the frog's gastrocnemius, for example, is such as to make their natural crosssection oblique. In consequence, their differences of potential are not distributed as in a regular parallel-fibred muscle like the sartorius. In the gastrocnemius, owing to the peculiar insertion of the muscle fibres into the tendon, the upper end of the muscle is really the middle of the longitudinal section, while the lower end is the acute angle of an oblique cross-section. When the ends are connected with an electrometer, a strong current is observed flowing (outside the muscle) from the upper to the lower end.

Uninjured Muscle. - Prepare a sartorius muscle with extreme care to prevent injury. Connect the tendon (the natural "cross-section") and the longitudinal surface with the electrometer 
through a short-circuiting key. Note the position of the meniscus on the micrometer scale. Open the short-circuiting key.

The meniscus will move but little. It will not move at all, provided the muscle has not been injured; kut the difficulty of preparation is such that some difference of potential will probably appear.

Close the key. Injure the muscle by drawing a hot wire across one end. Open the key.
A strong demarcation current will appear.

Stimulation by Demarcation Current. - 1. Make a nerve-muscle preparation (sciatic nerve and gastrocnemius muscle). Let the nerve near the muscle touch a cross-section of the sartorius. Now let the end of the nerve fall on the longitudinal surface near the equator.

The gastrocnemius will contract; the nerve acts as a conductor between the positive longitudinal surface and the negative cross-section.

It should be pointed out that the conclusion here drawn is not entirely free from criticism. The muscle is a conductor as well as the nerve, and may close the demarcation current of the nerve, as the nerve may close that of the muscle. Thus it is possible that the nerve is stimulated by its own demarcation current. The former explanation is the more probable. 
2. Place non-polarizable electrodes on the longitudinal surface and cross-section of the sartorius. Fasten the wires of the stimulating electrodes in the binding posts of the non-polarizable electrodes. Drop the nerve of the nervemuscle preparation across the electrode points.

The gastrocnemius will contract when the nerve bridges the space from one electrode to the other, and thus completes the circuit between the longitudinal surface and cross-section of the sartorius.

3. Place a little 0.6 per cent solution of sodium chloride in a porcelain dish. Fasten one end of the sartorius gently between two pieces of cork in the jaws of the muscle clamp. Bring the muscle over the saline solution. Make a fresh clean cross-section, and lower the clamp on its stand until the cross-section dips (not too far) into the solution.

The muscle will twitch. The twitch will pull the end of the muscle out of the solution. When the muscle relaxes, the contact between positive longitudinal surface and negative cross-section is once more made by the saline solution, the current of rest flows from the point of higher to the point of lower potential, and again stimulates the muscular tissue through which it passes. Thus the muscle is stimulated by its own cur- 
rent. A long series of contractions may be secured. Other liquid conductors will serve. When the solution touches only the cross-section, there is no contraction.

4. Prepare a fresh sartorius muscle with bony attachments. Fasten the pelvic end in the muscle clamp. Make a fresh cross-section in the first sartorius. Hold the tibial end of the second muscle in such a way that the muscle lies horizontally with its upper surface somewhat concave. Against this surface bring the fresh cross-section of the first sartorius. The longitudinal surface will naturally also touch to some extent.

The second muscle will close the circuit between longitudinal surface and cross-section of the first, and, if very irritable, both muscles will contract.

Interference between the Demarcation Current and a Stimulating Current; Polar Refusal. - Connect a dry cell through an open key with the 0 and 1 metre posts of the rheochord (Fig. 46). Mount two non-polarizable electrodes, and connect them through a pole-changer (with crosswires) to the positive post and slider of the rheochord. Tie a thick cotton thread to the foot of the positive electrode in such a way that the thread shall hang down in a small loop. 
Let a sartorius muscle rest on a clean glass plate. Make an artificial cross-section by drawing a hot wire across the muscle near the pelvic end. Pass the loop of thread on the positive electrode over the muscle about $5 \mathrm{~mm}$. from the thermal cross-section. Let the negative electrode rest on the cross-section. Arrange the rheochord for weak currents. Moisten the electrodes with normal saline solution. Close the key.

The usual closing contraction will be absent (polar refusal).

Note that the galvanic current is now passing through the muscle in an atterminal direction, $i$. e. towards the injured portion (admortal), while the demarcation current is passing through the muscle in

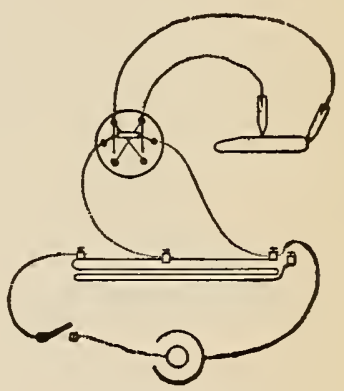

Fig. 46. the opposite direction. The two currents more or less compensate each other. Hence, the absence of the closing contraction. Observe, also, that opening the key will break the galvanic circuit, but that the circuit for the demarcation current will still be closed - through non-polarizable electrodes and rheochord.

Open the key.

An opening contraction will take place, obviously because the muscle curreut is no longer compensated. 
Reverse the pole-changer, so that the anode lies at the cross-section. Open and close the galvanic current.

Contraction will take place at closure only. The electrode at the cross-section again refuses.

2. Compensation Method. - The electromotive force of a current of injury may be expressed in fractions of a Daniell cell, or any other constant element, by bringing into the same circuit with the current of injury, but in an

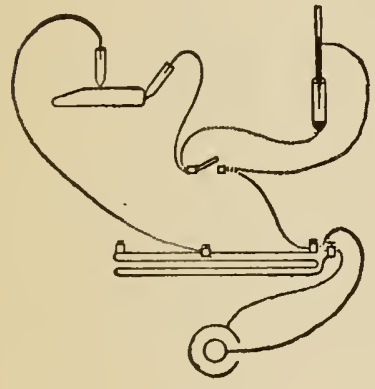

Fig. 47. opposite direction, so much of the current from the cell as will exactly balance the current of injury, $i$. e. so much as will keep the meniscus of the electrometer from moving in either a positive or negative direction when connected with the circuit.

Prepare a sartorius muscle. Connect a Daniell cell with the 0 and 10 metre posts of the rheochord. Connect the capillary electrometer to a closed short-circuiting key. From the post joined to the capillary lead to the 0 post of the rheochord. Connect the remaining post of the key to a non-polarizable electrode placed on the crosssection of the muscle. Join the slider of the rheochord to another non-polarizable electrode 
placed on the equator of the muscle (Fig. 47). Bring the slider to the zero post. Bring the meniscus into the field. Note its position on the micrometer scale. Open the short-circuiting key. When the meniscus comes to rest, move the slider along the rheochord until the meniscus returns to its original position. Read the number of millimetres between the positive post and the slider. This number divided by 10,000 is the fraction of the electromotive force of the Daniell cell (1.1 volt) necessary to balance the current of injury of the muscle (from 0.035 to 0.090 volt).

\section{Demarcation Current of Nerve}

Place non-polarizable electrodes on the crosssection and longitudinal surface of a long piece of sciatic nerve. Conrect the electrodes through a short-circuiting key with the electrometer. Bring the meniscus into the field and open the short-circuiting key.

The meniscus will move in a direction indicating a current in the nerve from cross-section to longitudinal surface, as in muscle.

Measure the electromotive force of this demarcation current.

The demarcation current is much weaker in nerve than in muscle, being in the former about 
0.025 volt, as against about 0.060 volt in muscle. The demarcation current of muscle is maintained in force for a long time, whereas that of nerve diminishes rapidly. The nerve current is restored on making a fresh cross-section.

The demarcation current from the cut branches of a nerve may reach electrodes placed on the main trunk, and thus confuse the electrometer measurements. To this same cause must be ascribed the increased irritability observed in the main trunk in the neighborhood of branches; the irritability is raised by the demarcation current of the severed branch.

Nerve may be stimulated by its own Demarcation Current. - On a glass plate make a U shaped wall of normal saline clay, each limb about $1 \mathrm{~cm}$. long and 3 or $4 \mathrm{~mm}$. wide. Carefully remove the moisture between the clay walls with filter paper. Lay the longitudinal surface of the nerve of a nervemuscle preparation on one limb of the $\mathrm{U}$, and with a glass rod let the cross-section fall on the other limb.

When the circuit between the cross-section and the longitudinal surface is completed by contact with the clay, the demarcation current will stimulate the nerve, and the resulting nerve impulse will cause the muscle to contract.

Other Examples. - The dropping of the central end of the severed vagus nerve into the wound from which it was lifted has caused the slowing 
of respiration, presumably by the stimulation of the nerve through the closure of its own demarcation current by the lymph or blood, though the possible influence of demarcation currents from the wounded tissues cannot be forgotten. Definite results, such as inhibition of the heart, have not been observed to follow the closure of the current of the peripheral segment. To avoid any chance stimulation from the closure of the de marcation current, nerves are sometimes severed physiologically by freezing, - a process which not only does not stimulate, but which does not destroy permanently the conductivity; the latter returns upon the restoration of the nerve to normal temperature.

The olfactory nerve of the pike shows a strong demarcation current, as does the optic nerve.

\section{Hypotheses Regarding the CaUsation of the Demarcation Current}

Make artificial cross-sections in a sartorius muscle, and test the difference of potential between the longitudinal surface and a cross-section with the electrometer. Divide the muscle, longitudinally, and make fresh cross-sections; test the difference of potential again.

However small the muscle prism may be 
made, the longitudinal surface will still be positive to the cross-section.

Molecular Hypothesis. - The fact that the smallest possible muscle prism is still positive on the longitudinal surface, and negative on the cross-section, suggested to DuBois-Reymond that muscle (and nerve) are composed of electrical particles or molecules something like the molecules of a magnet. A magnet has two poles, and, however it may be divided, the pieces still

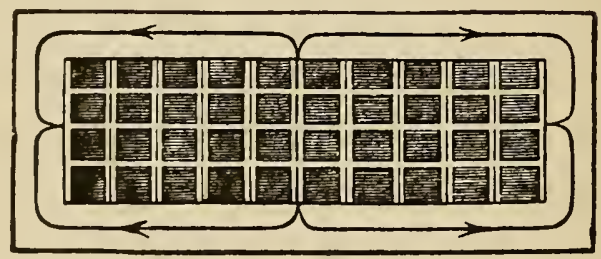

Fig. 48. Scheme of the inyomeres in a parallel-fibred muscle (Rosenthal).

possess a north and a south pole. The magnet is therefore believed to be composed of molecules, each possessing a north and a south pole. These molecules lie with the north poles all pointing in one direction, the south poles in the other. The structure of muscle favors, in a measure, a similar hypothesis; for it is known that a striated muscle consists of fibrillæ, each of which is composed of a row of particles arranged in quite regular fashion. The electromotive molecules, or 
myomeres, may be conceived to be positive on their longitudinal surfaces, and negative on their cross-sections (Fig. 48). They are assumed to have their negative surfaces turned towards the ends of the muscle or nerve, and the positive equatorial region turned towards the longitudinal surface. A non-electric conducting substance surrounds them. An electrode placed on the longitudinal surface would touch only the positive sides, while an electrode placed on the cross-

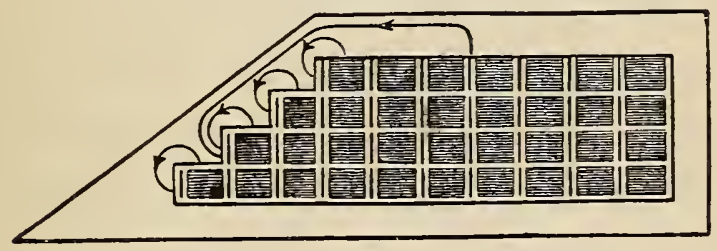

Fig. 49. Scheme of myomeres in an oblique section (Rosenthal).

section would touch only the negative poles. However small the muscle prism was made, the relation would still be the same. Thus the distribution of potentials would correspond with that actually observed.

When the cross-section is oblique, the myomeres at the cross-section are exposed as shown in Fig. 49, and the currents which pass from the longitudinal surface of each myomere to its crosssection are added to the main currents passing 
from the longitudinal surface to the cross-section of the whole muscle. The region of maximum positive potential is thereby brought towards the obtuse angle of the oblique cross-section, and the region of maximum negative potential is displaced towards the acute angle, as actually observed.

When it was found by Bernstein, Hermann, and others, that uninjured muscle showed no difference of potential, DuBois-Reymond assumed that in the natural, uninjured state the end of the muscle in contact with the tendon (the "natural cross-section") is composed of a layer of molecules which have their positive instead of negative surface turned towards the tendon.

The highly artificial and complicated structure which DuBois was compelled to erect on this foundation in order to explain all the electrical phenomena of living tissue, cannot be discussed here. The chief argument against the molecular theory of muscle and nerve currents is that the phenomena can be explained in a simpler way.

Alteration Theory. - This hypothesis, in the making of which Hermann and Hering have been especially active, explains the electromotive forces of nerve and muscle by alterations in the chemical composition of the tissue at the 
cross-section. When the cross-section is made, the tissue next the section passes through the series of catabolic changes which constitute muscle death; carbon dioxide is given off, lactic acid is developed, a soluble proteid is converted to a less soluble form, etc. The contact of this dying layer with the uninjured tissue is believed to create a difference of potential. The potential difference, therefore, appears at the demarcation between dying and uninjured tissue, - hence the term "demarcation current." The action current finds its explanation in the chemical changes acçompanying contraction. It would be interesting to consider here the parallel between the chemical transformations in contrac. tion and those which usher in the death of the muscle, but we must be content with mentioning the apparently close relationship. In its most general form, the alteration hypothesis rests on the fact that living substance is everywhere the seat of constant constructive and destructive changes. Where these are nearly in equilibrium, as, for example, in the resting uninjured muscle, the tissue is equipotential; where, on the contrary, either form of chemical change has the upper hand, as in the explosion which we term contraction, and in dying muscle, it is assumed that a difference of potential is created. 
For many years the weight of physiological opinion has been largely on the side of the alteration hypothesis; but it would be unsafe without further evidence to decide finally against the molecular theory.

\section{Action Current of Muscle}

The demarcation current (current of injury, current of rest) just studied has been shown to be due to the injury of the tissue. We have now to examine the electromotive forces which appear when a nerve or muscle becomes active.

1. Rheoscopic Frog. - Make two nerve-muscle preparations, $A$ and $B$. Let the nerve of $B$ rest on muscle $A$. Stimulate the nerve of $A$ with single induction shocks, and with the tetanizing current.

Muscle B will contract once for each contraction of $A$. The current of action of muscle $A$ stimulates the nerve of $B$.

Secondary contraction can take place also from muscle to muscle, but only under circumstances that suggest increased irritability, as, for example, through partial drying. No secondary contraction has been secured from voluntary muscular contraction.

2. That the stimulus to the nerve of the rheoscopic muscle is really an electrical current, is 
shown by the capillary electrometer. Place muscle $\mathrm{A}$ in the moist chamber upon two nonpolarizable electrodes. Let the tendon rest on one electrode and the equator on the other. Lead

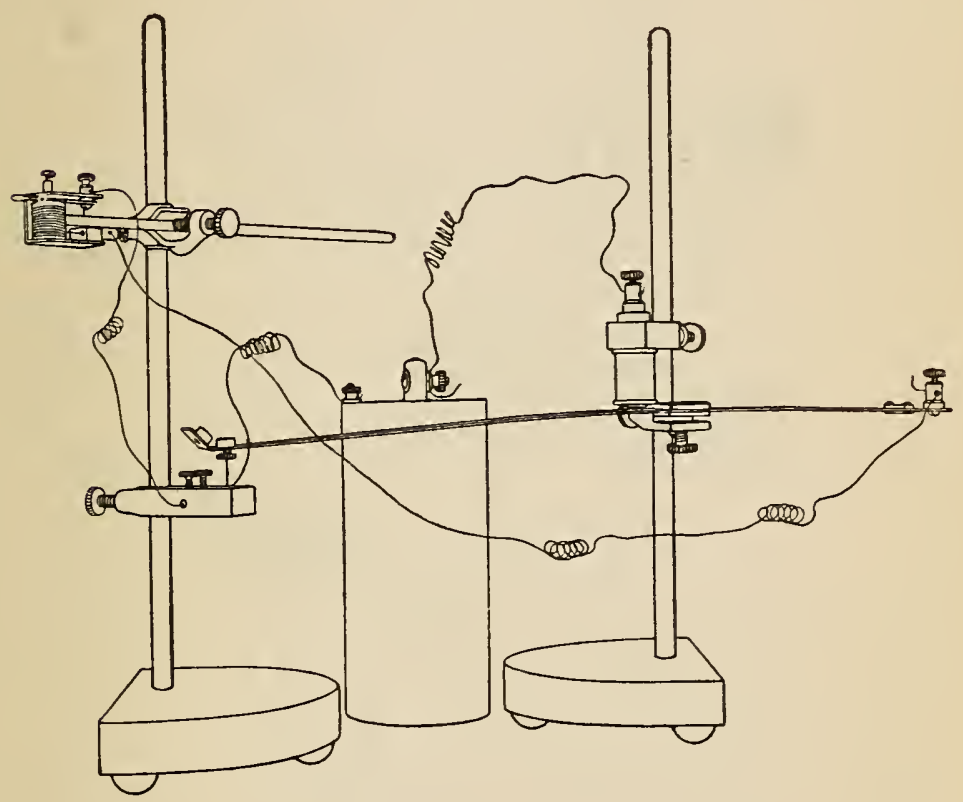

Fig. 50. The vibrating interrupter. A platinum wire on the end of a steel spring dips into a mercury cup. By varying the length of the spring, contacts from once a second to more than one hundred per second may be secured.

from the nom-polarizable electrodes through a closed short-circuiting key to the capillary electrometer (the tendon should be connected with 
the capillary). Lay the nerve on stimulating electrodes. Connect the latter with the secondary coil of an inductorium arranged for single induc-

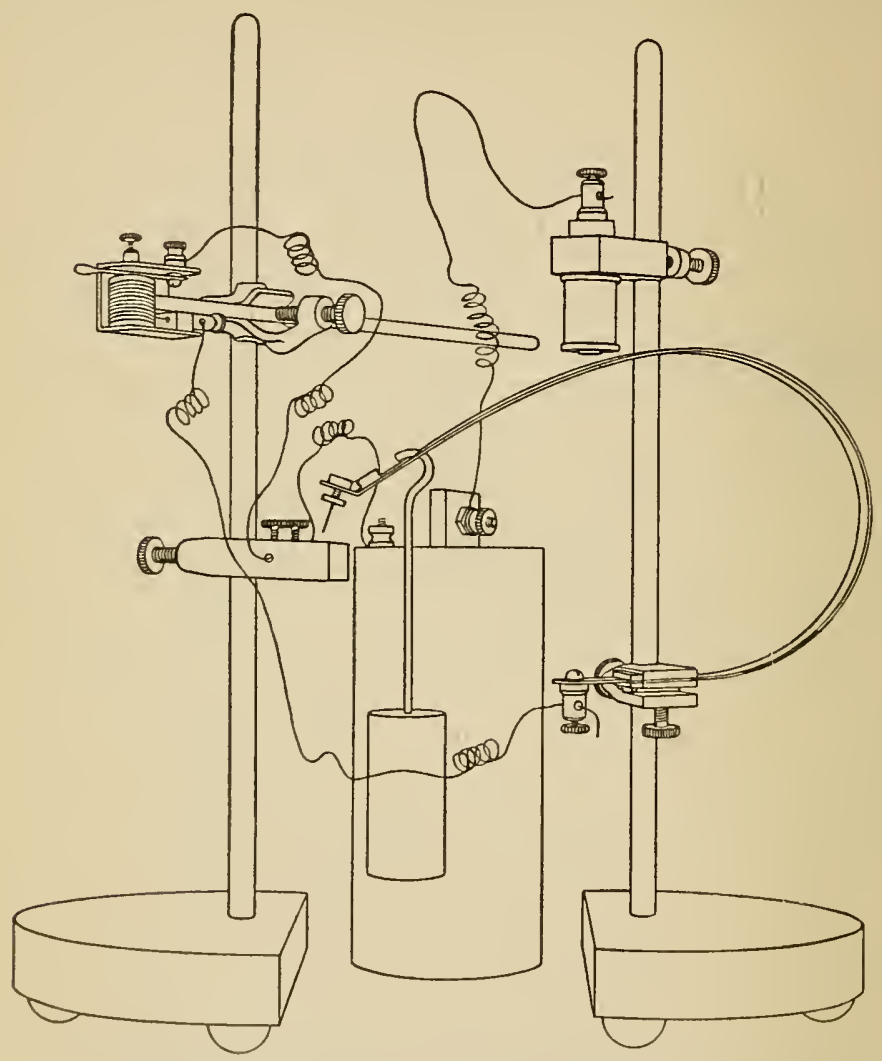

Fig. 51. The vibrating interrupter arrangerl to make one contact per second.

tion currents. Place the vibrating interrupter (Figs. 50, 51) in the primary circuit. Bring the meniseus into the field. Open the short-eireuiting 
key. The meniscus will be displaced by the demarcation current. When the meniscus has come to rest, stimulate the nerve with single and repeated induction currents.

With each stimulus there will be a negative variation (action current) of the demarcation current.

When the number of stimuli per second passes a certain point, which differs with different individuals, the hitherto separate excursions of the meniscus will be fused, and a gray blur will appear at the end of the vibrating column. Movements of this rapidity may of course be studied by photographing them on sensitive paper moving rapidly enough to draw the fused image out into a line in which its component oscillations are each distinct, or they may be observed directly by the stroboscopic method.

\section{The Action Current in Tetanus; Stroboscopic} Method. - 1. If a piece of thin black paper about $1 \mathrm{~cm}$. square is fastened vertically on the end of the electro-magnetic signal lever, and the signal placed in the primary circuit of the inductorium arranged for tetanizing currents, the piece of paper will move each time the primary current is made or broken by the vibrating hammer of the inductorium. The movement is so rapid that the paper seems stationary and a gray haze appears on its upper and lower border. 
Connect the electrometer with the secondary coil of the inductorium, and bring the vibrating meniscus into the field.

Bring the stroboscopic paper next the acid reservoir of the electrometer at such a height that the edge of the meniscus shall be seen through the gray blur. The meniscus will no longer appear blurred, but will be as sharp as if the mercury were stationary. This appearance is produced only when the stroboscopic paper and the object seen by its aid have the same periodicity of vibration. If the periodicity of the vibrations is unequal, interference results, and from this interference the rate of vibration of the observed body can be calculated. For example, if the observed body shows three vibrations per second, when observed through the stroboscope, its rate is three more per second than that of the stroboscope.

In the present instance, the meniscus remains apparently at rest. The number of action currents is therefore identical with the number of stimuli.

2. Rheoscopic Muscle Tetanus. - The same method may be applied to the analysis of the rheoscopic tetanus in the rheoscopic muscle.

Place two nerve-muscle preparations in the moist chamber. Place the tendon of muscle $\mathrm{B}$ 
on one electrode and the longitudinal surface on the other, and connect them through a shortcircuiting key with the electrometer. Lay the nerve of $\mathrm{B}$ on muscle $\mathrm{A}$. Place the nerve of $\mathrm{A}$ on electrodes connected with the secondary coil (the coil should be well over the primary). Bring the meniscus into the field, and open the short-circuiting key. Place the stroboscope, still in the primary circuit, near the meniscus. Tetanize the nerve of $\mathrm{A}$.

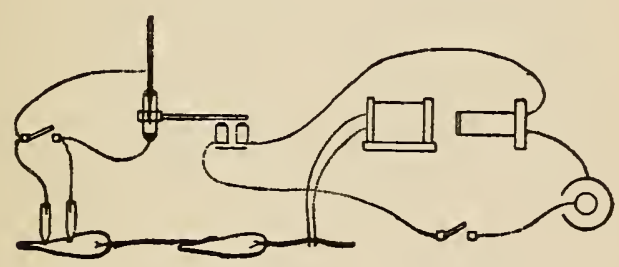

Fig. 52.

For each stimulus received from nerve $A$, muscle A contracts; the contractions are so frequent that they fuse into tetanus. At each contraction of $\mathrm{A}$, its current of action stimulates the nerve of $\mathrm{B}$, and $\mathrm{B}$ also contracts. At each contraction of $\mathrm{B}$, the action current displaces the meniscus, which falls therefore into very rapid oscillation. Observe the meniscus through the stroboscope. It will seem to be standing still. 
Thus the apparent continuous contraction of muscle B is in reality a series of simple contractions, as stated, corresponding in number to the make and break currents of the inductorium. For each contraction there is one action current in each muscle. ${ }^{1}$

When a muscle and its nerve are removed without injury to the muscle, electrodes placed on the latter will show no difference of potential, as already stated (page 289). Stimulation of such a muscle through its nerve causes a current of action to start at the point at which the nerve enters the muscle fibres. The contraction wave begins also at this point, as may be shown very beautifully by "fixing" the contraction in the muscles of certain insects by plunging the contracting muscle into a solution which arrests and "sets" the fibre instantly. In such cases fibres will be found in which the contraction wave is caught at its beginning in the neighborhood of the nerve end-plate.

The action current, beginning at the entrance of the nerve into the muscle fibre, passes in both directions along the fibre. As may be shown with the differential rheotome, or by photographing the meniscus of the capillary electrometer,

1 'The experiment also clemonstrates that the meniseus has no after vibrations, but follows unerringly the changes of potential. 
the current is diphasic. In the first phase, the current is directed away from the nerve, in the second phase, towards it. In extirpated muscle, the second phase is much weaker than the first. In normal muscle in situ (human muscle), this difference or decrement does not appear.

The direction of the current obtained with the electrometer from the whole muscle is determined by the position of the electrodes with reference to the nerve equator, namely, a transverse line drawn at the mean distance from the entrance of all the nerve fibres. Points nearer the equator are negative to points further away.

Action Current of Human Muscle. - Cover the brass electrodes with cotton saturated with saline solution, and connect them with an inductorium arranged for tetanizing currents. Close the short-circuiting key of the secondary coil. Tie about each of the non-polarizable electrodes a piece of well washed candle-wick a foot long. Saturate the wick with sodium chloride solution. Place one of these electrodes around the forearm near the elbow, the other around the wrist. (The nerve equator lies about the upper third of the forearm.) Connect the electrodes through a short-circuiting key with the capillary electrometer. Place the brass 
electrodes over the brachial plexus in the axilla. Bring the meniscus into the field. Open the short-circuiting key leading to the electrometer. If the meniscus is displaced by a skin (secretion) current bring it back by means of the pressure apparatus. Set the inductorium in action. Open the short-circuiting key of the secondary coil, thus stimulating the nerves.

The meniscus will be displaced by an action current.

Action Current of Heart. - 1. Expose the heart of a frog (page 112). Lay the nerve of an irritable nerve-muscle preparation on the beating ventricle.

During diastole, the rheoscopic muscle will be quiet; at each systole, it will contract.

2. Tie a cotton thread one inch long about the foot of each non-polarizable electrode, and let the ends, wet with normal saline solution, rest on the beating heart, one on the base, the other on the apex. These electrodes will follow the movements of the heart. Connect the electrodes through a short-circuiting key to the electrometer.

During the diastole, the meniscus will remain at rest. At each beat of the ventricle, the meniscus will move; first in a direction indicating that the base is negative to the apex, and then 
in the opposite direction. The action current passes over the heart from base to apex.

These experiments show not only that there is an action current at each systole of the heart, but are evidence also that the resting heart muscle is iso-electric ( $i$. e. of uniform potential).

The Action Current precedes the Contraction. Expose the heart. Fasten a very fine copper wire to the ventricle; the end of the wire may be thrust through the tip of the ventricle. Mount

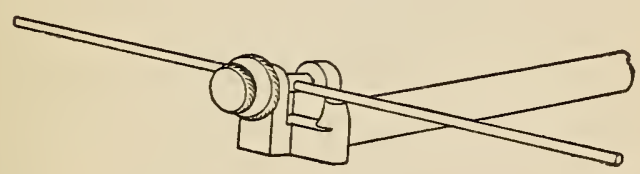

Fig. 53. The Heart Lever.

the heart lever (Fig. 53) on a stand so that the writing point will write on the smoked paper of the kymograph. Bring the free end of the wire to the heart lever, on which it may be fastened with a drop of colophonium cement. Make a nerve-muscle preparation. Fasten the femur in the upper side of the muscle clamp, at right angles to the long axis of the clamp. Bring the latter near the heart lever, so that the nerve may rest on the ventricle. Fasten the tendon Achilles to the muscle lever by a thread which passes over the pulley on the axis of the lever before being 
secured to the lever. Thus the muscle, though below the lever, will pull it upwards when contraction takes place. Let the two writing points be in the same vertical line. Start the drum at rapid speed. Two curves will be recorded: one by the contraction of the ventricle, the other by the rheoscopic muscle, stimulated to contract by the action current. The contraction of the rheoscopic muscle will slightly precede the contraction of the ventricle.

Current of Action of Human Heart. - Place normal saline solution in two beakers. In each let the foot of a non-polarizable electrode dip. Connect the electrodes through the usual shortcircuiting key with the electrometer. Bring the meniscus into the field. Let an assistant place a finger of each hand in the saline solution.

When the short-circuiting key is opened the meniscus will be displaced by the skin (secretion) current. Careful observation will show also a periodic variation synchronous with the systole of the heart.

The diphasic eharacter of the action current of the heart, shown so well by the eapillary electrometer to the unaided eye, appears even more clearly when the movenents of the meniscus are recorded by projecting them on a quickly moving photographic plate. By photography, too, the di- 
phasic character of the action current in the more rapidly contracting skeletal muscle is inade visible, and the form of the action current wave recorded. Before the capillary electrometer was used for

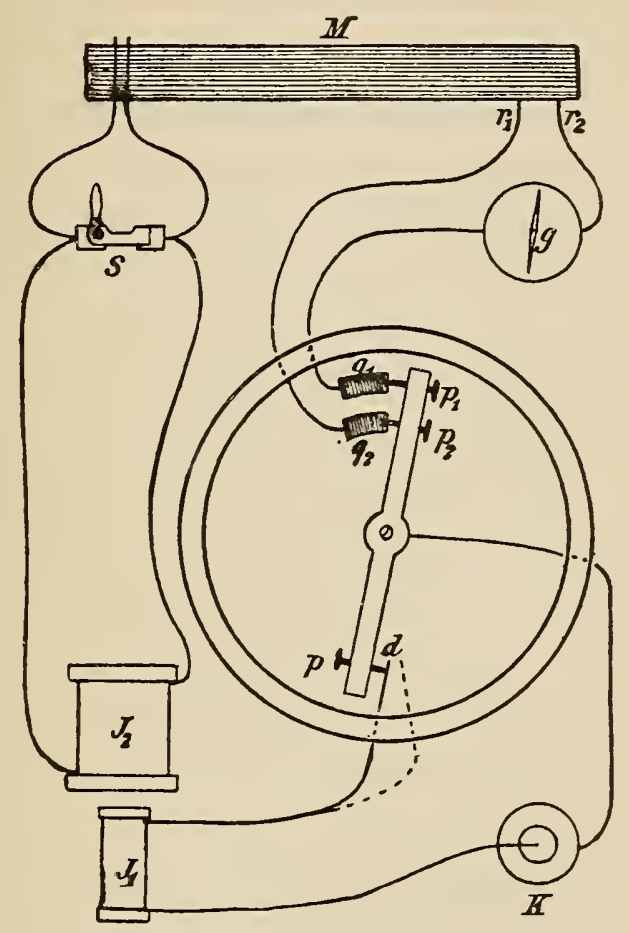

Fig. 54. Scheme of differential rheotome.

this purpose, the differential rheotome of Bernstein was employed. This celebrated invention consists of a wheel which revolves at uniform speed and carries contacts by which the primary circuit of an inductorium and a galvanometer 
circuit may be made. By means of the inductorium, the muscle is stimulated at one end. The galvanometer records the current of action by means of electrodes placed at the other end of the muscle. The position of the galvanometer contact on the wheel can be shifted nearer to or farther from the stimulating contacts; thus the interval between stimulation and the making of the galvanometer circuit may be chosen at will, and the electromotive force at any point in the action wave registered. By repeatedly changing the interval, the several portions of the wave can be investigated successively, and the results plotted. With Hermann's rheotachygraph, the whole electrical change may be recorded at one time. In this instrument the stimulating contacts revolve rapidly, and the galvanometer contact less rapidly, so that the interval between stimulation and the closure of the galvanometer continually alters. The effect of the electrical change on the galvanometer is thus prolonged so that the galvanometer mirror is able to follow it.

The results from these different methods agree in showing that the electrical change sweeps over the muscle (and nerve), in the form of a wave at a rate, in frog's muscle, of about three metres per second. The duration of the wave is from 0.003 .3 to 0.0040 second. The ascent is 
quicker than the descent. The latent period is probably absent; the process begins as soon as the stimulus reaches the muscle. The electromotive force of the action current for a single contraction of the frog's gastrocnemius is about 0.08 volt.

Direct stimulation of the whole of a normal uninjured muscle produces no action current whatever, because the whole muscle becomes active at the same moment.

\section{Action Currejt of Nerve}

1. Negative Variation. - Sever the nerve of a nerve-muscle preparation close to the muscle, and lay the nerve in the moist chamber on nonpolarizable electrodes placing the equator on one and a cross-section on the other. Lead them through a short-circuiting key to the capillary electrometer. Place a second pair of non-polarizable electrodes near the other cross-section of the nerve. Connect this second pair to the secnndary coil of an inductorium. Connect the primary coil through a key and the wheel interrupter with a dry cell. Bring the meniscus into the field. Open the short-circuiting key. The meniscus will be displaced by the demarcation current. Stimulate the nerve with induction shocks at different rates. 
A negative variation will be observed each time the nerve is stimulated.

2. The current of action is not dependent on the electrical stimulation, but is an expression of the changes in the nerve which constitute the nerve impulse. It follows mechanical as readily as electrical stimulation.

Lead to the capillary electrometer from nonpolarizable electrodes placed on the longitudinal surface and cross-section. Note the position of the meniscus. Stimulate the nerve mechanically by snipping the end with the scissors.

There will be a negative variation as before.

Positive Variation. - The direction of the current of action is not always opposite to that of the demarcation current. Biedermann obtained a current in the positive direction on stimulating the nerve to the abductor muscle in the lobster. In the tortoise, the cardiac auricle may be cut away from the sinus, without injury to the coronary nerve, which in this animal carries to the auricle the cardiac fibres of the vagus. After this operation, the auricle and ventricle remain motionless for a time. In a heart thus prepared, Gaskell made a thermal cross-section by immersing the tip of the auricle in hot water, and led the demarcation current to a galvanometer. The stimulation of the vagus in the neck - the 
heart still resting - caused a marked increase in the demarcation current, in other words, a positive variation. No visible change in the form of the heart was observed.

Positive After Current. - Compensate the demarcation current of nerve by the method described on page 294. When compensation is secured, note the position of the meniscus on the scale, and tetanize the nerve. The meniscus will be displaced by the current of action. Note the direction of the current. Break the stimulating current. The meniscus will return to and pass the position which it held when the demarcation current was compensated, showing thus a current opposed in direction to the action current.

The positive after current is absent in weakened or fatigued nerves.

Contraction secured with a Weaker Stimulus than Negative Variation. - Place the non-polarizable electrodes on the longitudinal surface of the nerve of a nerve-muscle preparation. Connect them through the usual short-circuiting key with the electrometer. Bring the meniscus into the field. Arrange the inductorium for break currents. Place the secondary coil some distance from the primary. Stimulate the nerve in the extrapolar region. Approach the coils 
until the threshold value is reached and the muscle contracts.

At the threshold value of inuscular contraction, the current of action in the nerve will not yet be demonstrable. The coils must be still nearer together before the action current becomes visible.

This experiment has a certain suggestive value. It would not, however, be safe to conclude from it that the action current is not an essential part in the passage from the resting to the active stage. The failure to recognize the action current probably lies in the method.

Current of Action in Optic Nerve. - Place two non-polarizable electrodes in the moist chamber, and connect them through a short-circuiting key with the capillary electrometer. Remove the eye of the frog, together with a portion of the optic nerve, and lay the preparation on the electrodes in the moist chamber, letting the edge of the cornea touch one electrode and the optic nerve the other. Cover the electrodes and the preparation with a black pasteboard box or other opaque screen to shut off the light. Note the position of the meniscus in the field of the microscope. Open the short-circuiting key. A demarcation current from the injured optic nerve to the cornea will be indicated. Re- 
move the box so that light shall fall on the retina.

The demarcation current will undergo a negative variation.

Shut off the light by replacing the box.

There will now be a positive variation.

Currents of action have also been demonstrated in the central nervous system. Gotch and Horsley find that when the spinal cord of the monkey is severed, and non-polarizable electrodes are applied to the longitudinal surface and the cross-section, a negative variation of the current of injury appears whenever the cortex of the cerebrum is stimulated in the neighborhood of the fissure of Rolando, - the "motor" region. A considerable degree of localization in the cord is possible. It may be shown that the negative variation from the motor region of the cortex descends the cord chiefly in the crossed pyramidal tract, - a collection of white fibres in the lateral column of the cord near the gray matter. It is known from pathological evidence that the nerve impulse from the motor cortical cells passes through these fibres, and the demonstration of their negative variation justifies the hope that this method may be useful in determining the course of other nerve fibres in the brain and cord. 
Errors from Unipolar Stimulation. - Attention already has been called to the danger of unipolar induction currents entering the electrometer circuit in observations of the action current with the capillary electrometer or galvanometer (page 74).

Place a nerve in the moist chamber. Connect the capillary electrometer through a short-circuiting key with non-polarizable electrodes placed on the longitudinal surface and cross-section, about $5 \mathrm{~mm}$. apart. Let a wire connected with one pole of the secondary coil rest on the nerve about $2 \mathrm{~cm}$. from the non-polarizable electrodes. Open the short-circuiting key. When the meniscus has come to rest, set the inductorium in action.

If the meniscus remains at rest, bring the secondary coil nearer the primary, until unipolar effects appear.

\section{Secretion Current}

Secretion Current from Mucous Membrane. Remove the skin from the lower jaw of a frog, the skull of which has been cut away. Be very careful not to touch the tongue with metal instruments or with fragments of skin. Make a normal saline clay electrode about $1 \mathrm{~cm}$. square and $3 \mathrm{~mm}$. thick on the glass of the cork clamp near the 
cork. Lay the denuded jaw on the glass, and turn the tongue forward with a glass rod until the tip can be secured in the clamp. Avoid all roughness. 'The normally upper surface of the tongue will now rest on the clay. Bring one non-polarizable electrode into contact with the clay, and let the other touch the upper (normally lower) surface of the tongue. Connect the electrodes through an open key with the capillary electrometer. Bring the meniscus into the field, and note its position on the micrometer scale. Close the key.

A strong difference of potential will be shown. The normal under surface is usually positive towards the normal upper surface.

The difference of potential thus demonstrated is probably chiefly due to secreting glands in the mucous membrane. If the "secretion current" is compensated after the general compensation method described on page $29 \dot{4}$, and the glossopharyngeal nerve then stimulated, the electrometer will show an electromotive force, in a direction opposite to the original difference of potential, - in other words, a "negative variation."

Negative Variation of Secretion Current. - Place a frog curarized until voluntary motion is just paralyzed back uppermost on the frog board. Strip the skin from one thigh, and expose 
the sciatic nerve of this side. Place non-polarizable electrodes on the bare muscle of the thigh and on the skin of the leg. Connect the electrodes to a rheochord arranged for compensation by the bridge method, as shown in Fig. 47. Place the capillary electrometer in a short circuit. Bring the meniscus into the field, and note its position. Open the short-circuiting key. Move the slider along the wire until the meniscus returns to its original position. Now stimulate the sciatic nerve with the tetanizing current.

A negative variation will be seen. If the skin current was slight, the variation may be positive.

The greater part of the skin current is doubtless a secretion current, but not all. Weak currents have been obtained from skin devoid of glands, for example, the eel's skin. Hermann attributes this current to the degeneration which accompanies the change of the nucleated cells of the corium to the dead scales of the outer epidermis.

A strong secretion current may be obtained from the skin of the foot (cat). On stimulation of the sciatic nerve, the current is increased (positive variation).

In the submaxillary gland, the hilus is positive to any point on the external surface of the gland. Stimulation of the chorda tympani nerve, secre- 
tory fibres from which are supplied to the gland, causes the surface to become still more negative, $i$. e. the secretion current is increased (positive variation). Stimulation of the sympathetic, which also sends fibres to the gland, causes the secretion current to lessen (negative variation).

\section{Electrotonic Currents}

It has already been showu that the irritability and conductivity of the nerve are altered by the

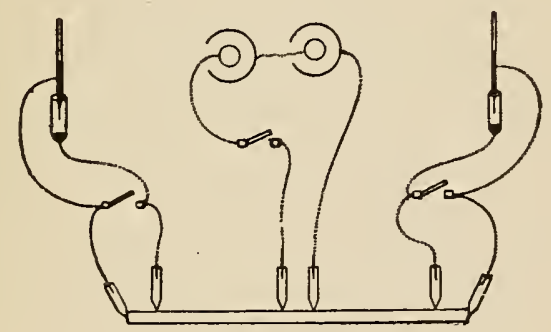

Fig. 55.

galvanic current. So also are the electromotive properties.

Place one pair of non-polarizable electrodes near the middle of a long piece of extirpated nerve, and one other pair at each end, on the cross-section and longitudinal surface as in Fig. 55. Connect the middle pair through a key with two dry cells. Connect each of the other pairs through a short-circuiting key with a 
capillary electrometer. Let one observer watch each meniscus, while a third experimenter manages the polarizing current. Note the position of each meniscus. Open the short-circuiting keys. In each electrometer, the meniscus will be displaced by the demarcation current. It should be noted that the demarcation currents are of opposite direction, flowing in the nerve from the cross-section towards the longitudinal surface. Make the polarizing current.

When the polarizing current enters the nerve, there will be a twitch in each electrometer, caused by the negative variation of the demarcation current; this may be neglected. Each meniscus will be displaced; on the side of the anode of the polarizing current, the demarcation current will be reinforced, but on the side of the cathode it will be diminished.

Thus the passage of the galvanic current through a part of the nerve has polarized the nerve on both sides of that part. The extrapolar region on the side of the anode becomes positive; the extrapolar region on the side of the cathode becomes negative; similar changes probably occur in the intrapolar region. In short, an electrotonic current is set up, having the same direction as the polarizing current. This electrotonic current angments the demarcation current 
on the side of the anode, but is opposed to that on the side of the cathode. It appears when any two points on the longitudinal surface are "led off" to the electrometer, and is entirely independent of the demarcation current.

The intensity of the electrotonic current depends on the intensity of the polarizing current. The greater the separation of the polarizing electrodes, the less the electrotonic effect, as might be expected from the great resistance of nerve. If this factor be excluded by placing in the circuit a much greater resistance than that of nerve, the electrotonic effect will be found to increase with the length of the intrapolar region. The electrotonic current is absent in dead nerves, in strongly cooled nerves, and in those ligated between the polarizing electrodes and the electrodes leading to the electrometer.

In muscle, the electrotonic currents are much stronger than in nerve.

Negative Variation of Electrotonic Currents; Positive Variation (Polarization Increment) of Polarizing Current. - Place the polarization electrodes near one end of the nerve. Connect them through a short-circuiting key with a dry cell. From the short-circuiting key lead to a capillary electrometer (Fig. 56). From the middle of the nerve lead off the electrotonic current through a short. 
circuiting key to a second capillary electrometer. Near the other end of the nerve place stimulating electrodes connected with the secondary coil of an inductorium arranged for tetanization. Make the polarizing current. Open the shortcircuiting key leading to the electrotonic electrometer, and note the position taken by the meniscus under the influence of the electrotonic current. Make the tetanizing current.

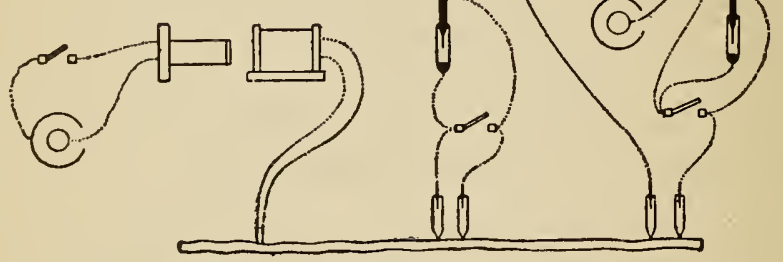

Fig. 56.

The strength of the electrotonic current will be diminished. At the same time the strength of the polarizing current will be increased (polarization increment).

These are in reality action currents.

The electrotonic currents are absent in nerves which lack a myelin sheath. This suggests that the myelin in some way divides the nerve into a core and a sheath. If a zinc wire connecting two electrodes is surrounded by a layer or sheath 
of saturated solution of sulphate of zinc, there will be no polarization, and the current will not spread to any extent beyond the electrodes. If, however, the wire is platinum instead of zinc, polarization will take place where the current passes from the electrodes through the electrolyte into and out of the wire, and the polarization may be recognized by connecting the extrapolar region with the electrometer as in the foregoing experiment. The resistance to the spread of the electrotonic current in a longitudinal direction is relatively slight, so that it passes almost instantly along the core.

In nerve, also, the greater resistance in the transverse direction (five-fold greater than the resistance in the longitudinal direction) would favor the spread of electrotonic currents lengthwise along the nerve.

Certain observations of Biedermann make it difficult to accept without reservation the simple physical explanation just offered. For example, the narcotization of a nerve with ether or chloroform causes the electrotonus to disappear a short distance from the electrodes, although still strongly present in their immediate neighborhood. These experiments cannot be discussed here, but they indicate that to the purely physical must be added a physiological electrotonus. 
The Electrotonic Current as a Stimulus. - As would naturally be expected, the electrotonic current may be an effective stimulus. Bring the end of an extirpated nerve A into contact with the distal portion of the nerve of a nerve-muscle preparation, B, as in Fig. 57, and place on the other end of A non-polarizable electrodes joined through a key to a battery of two cells. Make the galvanic current.

Muscle B will contract.

The galvanic current polarizes nerve $\mathrm{A}$, and

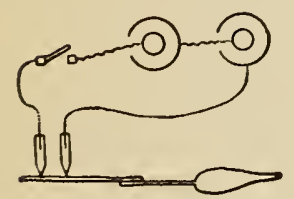

Fig. 57. the electrotonic current thereby set up passes into the nerve of $\mathrm{B}$ through the contact, and occasions in nerve $B$ an impulse which descends to the muscle and stimulates it to contract.

Paradoxical Contraction. - Expose the bifurcation of the sciatic nerve into tibial and peroneal branches. Polarize either of these branches. (The electrodes should not be placed too near the bifurcation.)

On making and breaking the polarizing current, the muscles supplied by each branch will contract.

In this instance, the extrapolar region of the branch polarized lies in part in the main trunk. The electrotonic current there spreads into the 
contiguous axis cylinders, among them those of the other branch.

\section{Electric Fish}

There are several species of fish which possess the power of discharging electrical currents when stimulated. The best known are Torpedo, a ray found on the coasts of Europe; Gymnotus, the electrical eel of South America; and Malapterurus electricus, a catfish found in the Nile and other African rivers. The electromotive force of these fishes is derived from a special organ placed beneath the skin. This electrical organ is bilateral and is formed of parallel plates. One side of each plate receives a branch of the electrical nerve, which in Malapterurus is a single great axis cylinder derived from a giant nerve cell. The side of the plate receiving the nerve becomes negative to the other side when the electrical organ is active; it behares like the negative plate of the ordinary cell. When the nerve is at rest, there is no difference of potential in the electrical organ. The discharge in the active state is periodic, and may rise to 200 per second. The electromotive force is considerable: in Torpedo, 30-35 volts, 5 volts for each cubic centimetre of the organ, 0.08 rolt for each plate. The fish itself 
is not injured by the current; its tissues are not easily excitable by electricity, though they respond readily to mechanical stimulation.

\section{Apparatus}

Normal saline. Bowl. Towel. Pipette. Glass plate. Sharp knife. Two dry cells. Four non-polarizable electrodes. Simple key. Capillary electrometer. Co-ordinate paper. Millimetre scale. Inductorium. Electrodes. Thirteen wires. Porcelain dish. Muscle clamp. Muscle lever. Stand. Cork. Rheochord. Normal saline clay. Filter paper. Wheel interrupter. Candle-wick. Electromagnetic signal. Pole-changer. Bent hooks. Black paper (stroboscope). Moist chamber. Large and small brass electrodes. Cotton. Common salt. Two beakers. Saturated solution of zinc sulphate. Cotton thread. Frog board. Heart-holder. Black box for covering retina. Bunsen burner. Glass slide. Cork clamp. Frogs. 


\section{III}

\section{THE CHANGE IN FORM}

THE change in form or the contraction of muscle is the most conspicuous of the several ways in which its energy is set free. It has already been shown that this change consists of a shortening of the contractile mass followed by a return to the original length. It is necessary now to determine whether the muscle becomes smaller on entering the active state or whether the alteration in form is simply a shifting - a translocation - of the muscular units.

\section{Volune of Contractiag Muscle}

Strip the skin from the hind limb of a frog. Hang the limb from the hooked electrode in the stopper of the volume tube (Fig. 58) and place the stopper loosely in the tube. Hook the electrode at the other end of the tube into the limb near the foot. Fill the tube absolutely full of boiled normal saline solution, slightly withdrawing the stopper for the purpose. Replace the stopper in the tube in such a way that all air 
bubbles shall be excluded. If the height of the

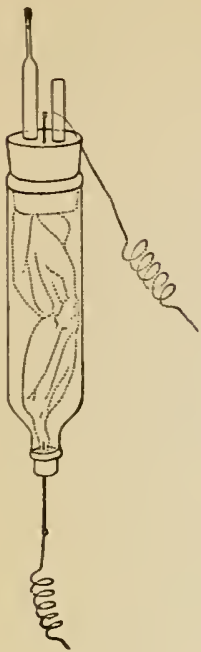

Fig. 5s. The volume tube. water-column in the capillary tube does not permit the meniscus to be readily observed, move the glass rod in the stopper in or out until the meniscus is adjusted. Connect the electrodes with the secondary coil of an inductorium arranged for single induction currents. Note carefully the level of the water in the capillary tube. Stimulate the muscle with a maximal break current.

The level of the water in the capillary will not change. The change in the form of the contracting muscle is not accompanied by a change in volume. ${ }^{1}$

\section{The Single Contraction or Twitch}

The change in the form of the muscle on entering the active state is usually studied from the graphic record made on a smoked surface by a writing lever the shorter arm of which is attached to the end of the muscle. Such a record, it should be remarked, gives the extent

1 This experiment must not be regitrded as cxcluding a very slight change in volume, becanse of the difficulty of expelling, by boiling or otherwise, all the air in the saline solution. 
and the time relations of the shortening, but not the thickening of the muscle. (See page 339.)

The Muscle Curve. Prepare a gastrocnemius muscle together with the distal third of the femur. Fasten the latter in the muscle clamp. Attach the tendo Achillis to the hook on the muscle lever by means of a fine copper wire which should be wrapped round the hook and the end then carried to the binding post on the muscle lever. Place a ten-gram weight in the scale-pan. Connect the posts on the clamp and the lever with the secondary coil of an inductorium arranged for maximal induction currents. In the primary circuit place an electromagnetic signal. Bring the writing points of the signal and the muscle lever against the smoked paper in the same vertical line. Start the drum at its most rapid speed. Stimulate the muscle with a maximal break current.

The muscle will shorten and then extend, marking a period of rising energy and a period of sinking energy. Note that the period of rising energy is shorter than the period of sinking energy. Close observation will show that the lever does not begin to move at the instant the muscle is stimulated, - there is here an interval or latent period.

The Duration of the Several Periods. - Turn to 
the right the screw at the top of the sleeve bearing the recording drum until the sleeve is raised from the friction bearing. The drum can now be "spun." Start the tuning fork vibrating, spin the drum, lay the writing point of the tuning fork on the smoked paper near the line traced by the electro-magnetic signal, and stimulate the muscle with a maximal induction current.

An interval will be found between the moment of stimulation (marked by the electromagnetic signal) and the beginning of contraction. This interval is the mechanical latent period. Measure its duration by means of the tuning fork curve. Measure also the duration of the period of rising energy and the period of sinking energy.

Helmholtz, who first measured the latent period of frog's muscle, found a mean duration of 0.01 sec., while the phase of rising energy measured 0.04 sec., and the phase of sinking energy 0.05 sec. More recent measurements by Tigerstedt and others have reduced the latent period given by Helmholtz to from 0.0025 to 0.005 sec. The interval observed grows less as the intensity of stimulation is increased from the threshold to the maximal value; further increase in intensity (supermaximal stimulation) causes no further diminution in the latent period. 
The period is shorter at high temperatures than at low, with maximal break induction currents than with make induction currents, with break induction currents than with closure of the galvanic current. Changing the load of the muscle is without effect on the latent period.

When the muscle is stimulated through its nerve the latent period is longer by about 0.002 sec. than when the electrodes are placed on the muscle itself (Bernstein), due allowance being made for the time occupied by the passage of the nerve impulse along the trunk of the nerve from the point of stimulation to the muscle. The additional time is taken perhaps in the passage of the impulse through the end plate into the contractile substance.

Griitzner has shown that the striated muscle tibres, particularly of vertebrates, differ in their histological elements. Some are rich in sarcoplasm, and when seen by transmitted light appear cloudy and granular; others have less sarcoplasm and are relatively translucent. This difference in structure is associated with a striking difference in the character of the contraction. The muscles composed chiefly of turbid fibres contract slowly, while "clear" muscles contract rapidly (compare page 178). Thus in the rabbit the duration of the contraction of the red soleus 
muscle, which is rich in sarcoplasm, is about 1.0 sec., while in the white gastrocnemius --a "clear" muscle - it is $0.25 \mathrm{sec}$. In the frog, the contraction period of the hyoglossus is 0.205 , the gastrocnemius 0.120 , and the gracilis 0.108 sec. (Cash). The latent period is longer in the red muscles. The amplitude of contraction is less in the red than in the white.

The mixture of quickly and slowly contracting fibres in the same muscle is sometimes obviously an advantage. Thus in certain bivalves the quick fibres in the shell-closing muscle close the shell rapidly, and the slow fibres keep it closed after the contraction of the quick fibres has ceased.

The form of the contraction is influenced by the mixture of fibres. The clear fibres reach their maximum shortening sooner than those rich in sarcoplasm. In some instances, indeed, the contraction curve may show two summits. These differences may perhaps explain the characteristic differences in the form of the contraction wave of different muscles, observed by Cash and others. The white fibres are more easily fatigued than the red. Thus the triceps humeri of the rabbit contracts at the beginning of stimulation like an unmixed white muscle (quickly), but later like a red muscle (slowly).

The Excitation Wave.-- Prepare a plate of 
cork one inch long and just narrow enough to be held in the Gaskell clamp (Fig. 26). Smoke a drum. Raise the drum off the friction bearing by turning to the right the milled screw at the top of the shaft. Fasten the end of a curarized sartorius muscle to the cork plate by means of two needles to the ends of which conducting wires are soldered. Place the preparation in the Gaskell clamp in such a way that the clamp shall compress the equator of the inuscle sufficiently to prevent the passage of a contraction wave from one part of the muscle to the other, but not sufficiently to prevent the passage of the excitation. Let a second pair of needle electrodes rest on the muscle near the upper side of the clamp. Fasten the clamp to the iron stand. Connect the two pairs of electrodes to the end cups of a pole-changer (without cross-wires), the side cups of which are connected with the secondary coil of an inductorium arranged for single maximal induction currents. In the primary circuit of the inductorium place the electromagnetic signal. Fasten the tibial end of the muscle to a muscle lever. Bring the writing point against the smoked surface exactly underneath the point of the electro-magnetic signal. "Spin" the drum slowly. Place the writing point of a vibrating tuning fork against the 
smoked paper below the recording levers. Stimulate the muscle with a maximal break current first through one pair of electrodes and then through the other. In each of the resulting curves measure the interval between stimulation and contraction (for method see page 184).

This interval will be longer when the muscle is stimulated farther from the portion the contraction of which is recorded. The difference is the time taken by the excitation to traverse the part of the muscle lying between the two pairs of electrodes. Measure the distance and calculate the speed of the excitation.

The nature of the excitation process is unknown. The current of action has been shown to precede the visible change in form of muscle. It is usually assumed to be a manifestation of the excitation process, but the precise relation between the two has never been ascertained. The speed of the excitation is the same as that of the contraction wave.

The Contraction Wave. - Remove from a curarized frog the long parallel-fibred muscles extending along the inner side of the thigh from the pelvis to the tibia. Let the preparation rest horizontally on a glass plate supported on a stand. With fine wire fasten near the axle of each of two heart levers a small piece of cork into which 
the point of a long pin has been thrust. Place the levers so that the head of the first pin rests on the muscle near one end, while the head of the second pin rests near the other end. Place needle electrodes at one end of the muscle. Bring the writing points of the two levers against a smoked drum in the same vertical line. Let a tuning fork write its curve near that of the muscle levers. Set the tuning fork vibrating. Let the drum revolve rapidly. Stimulate the muscle at one end with a maximal make induction current.

The lever near the point of stimulation will begin to rise before that farther away. Evidently the contraction starts at the point stimulated and spreads along the muscle in the form of a wave (compare pages 308 et seq.).

Determine the speed per second of the wave of contraction by measuring with the tuning-fork curve the time occupied by the wave in passing along the muscle from one lever to the other.

It is evident that a lever resting on a horizontal muscle will register the change in form of the cross-section on which the lever lies, while a lever attached to the end of a muscle suspended vertically will be moved by the change in form of all the cross-sections of which the muscle is composed. The curves secured by the two procedures 
are similar in form, but different in duration. The curve of thickening is shorter by the difference between the time taken by the contraction wave to pass over the single cross-section, on the one hand, and the whole length of the muscle on the other.

An extirpated muscle is apt to remain shortened after contraction. To bring muscles back to their original length it is usually necessary to weight them, or - as in the body - to submit them to the pull of antagonists. Even the weighted muscles may return very slowly and imperfectly to their normal length. This contracture, as it is termed, is seen especially in strong direct stimulation, in poisoning with veratrine, and as death comes on. Contracture is not the result of fatigue, for when the muscle is repeatedly stimulated contracture diminishes, instead of increasing. During contracture, the irritability of the muscle for stimulation through the nerve is diminished.

Relation of Strength of Stimulus to Form of Contraction wave. - Fasten the femur of a gastrocnemius preparation in the muscle clamp and attach the Achilles tendon to the muscle lever with a fine copper wire the end of which should be carried to the binding post on the landle of the lever. Connect this post and that on the 
muscle clamp with the secondary coil of the inductorium. Bring the writing point against the smoked drum. Stimulate the muscle with break induction currents of varying intensity and record the contraction curves.

It will be found that the contraction is longer with weak stimuli than with strong.

\section{Influence of Load on Height of Contraction. -} Attach a curarized gastrocnemius preparation to the muscle lever and bring the writing point against a smoked drum. Connect the binding posts on the lever and the muscle clamp with the secondary coil of the inductorium. Load the muscle with the lever and scale-pan only. With the drum at rest record the contraction on stimulation with a maximal induction current. Turn the drum by hand one millimetre. Place a one-gram weight in the scale-pan, and record the contraction produced by a make induction current of the same intensity as before. Continue to add gram weights and to record the contractions until ten one-gram weights have been placed in the scale-pan. Now increase the load each time by ten grams, recording the contraction after each increase, until the muscle is weighted with one hundred grams. (Care should be taken not to fatigue the muscle by stimulating it oftener than is necessary to obtain the record.) 
Within certain narrow limits the height of the contraction will be increased by the increase in the load. With increasing loads the height of contraction diminishes at first quickly, and then more slowly.

\section{Influence of Temperature on the Form of the} Contraction. - Prepare a gastrocnemius muscle together with its attachment to the femur. Fasten the femur in the "muscle warmer" (Fig. 59). Tie the end of a fine copper wire about ten centimetres long around the Achilles tendon. Bring the wire through the opening in the muscle warmer and fasten the wire around the pulley of the muscle lever. If the pulley is of metal the muscle lever should be supported on a stand separate from that bearing the muscle warmer or should be otherwise insulated. Connect the muscle warmer and the muscle lever with the secondary coil of an inductorium arranged for single induction currents. Fill a beaker with cracked ice and add a little salt. Immerse the muscle warmer in the beaker and support the latter on a suitable stand. Bring the writing point of the muscle lever against a smoked drum. Let the drum revolve at fairly rapid speed. Stimulate the cooling muscle at intervals of $5^{\circ}$ with a maximal break current. 
The Muscle Warmer. ${ }^{1}$ - A disk, supported by a rod, bears three pins (Fig. 59). One of the three pins is prolonged and bent at a right angle near its lower end. To the bend is fastened one end of the muscle under experimentation. About the other end is tied a fine copper wire which passes through a hole in the disk to reach a muscle lever. A second opening in the disk is provided with a short metal tube, in which a thermometer is held by a piece of rubber. The bulb of the thermometer may be placed on a level with the belly of the muscle. When these adjustments are complete, a glass cylinder is brought against the under surface of the disk, where it is held

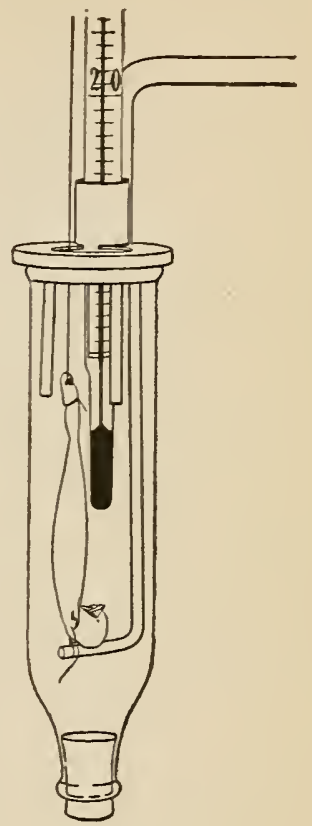

FIG. 59. The muscle warmer; an apparatus for studying the influence of temperature on muscular contraction.

in position by the "spring" of the three pins. A beaker or other vessel containing water is now placed beneath the cylinder and raised until the cyclinder is sufficiently immersed. The temperature of the muscle is altered by heating or cooling this

1 American Journal of Physiology, 1904, x, p. xliii. 
water. Direct electrical stimulation of the muscle may be made by connecting one electrode with the metal parts of the apparatus and the other with the copper wire attached to the upper end of the muscle.

Note that as the temperature falls the contraction curve becomes longer. The phase of rising energy is lengthened more than the relaxation. The earlier portion of the relaxation is lengthened less than the later; the muscle shows a tendency to contracture (see page 340 ).

Place fresh paper on the drum. Let the drum revolve very slowly. Place a lighted Bunsen burner under the arm of the muscle warmer. At intervals of $5^{\circ}$ stimulate the muscle with a maximal break current. Note the changes in the contraction.

The height of contraction is least at the freezing point of the muscle $\left(-5^{\circ}\right)$. It rises from the freezing point to $0^{\circ}$; falls from $0^{\circ}$ to $19^{\circ}$; increases to $30^{\circ}$, which is the maximum; from $30^{\circ}$ to $45^{\circ}$ diminishes again; and at $45^{\circ}$ the frog's muscle nsually enters into a state called rigor caloris; the muscle becomes opaque, inelastic, resistant to the touch, shortens very considerably, and undergoes chemical changes of great importance. The duration of contraction lessens with the rising temperature, being least at $30^{\circ}$. Above $30^{\circ}$ the duration remains approximately unchanged. The latent period is increased at low 
temperatures, diminished at high. Above $30^{\circ}$ the excitability to electrical stimuli diminishes steadily; it disappears almost entirely before rigor is reached.

Influence of Veratrine on the Form of the Contraction.- With a capillary pipette inject in the dorsal lymph sac 5 d̀rops of a 1 per cent solution of veratrine sulphate or acetate. After a few minutes, test for symptoms of veratrine poisoning by pinching the foot from time to time.

Soon the mechanical stimulation will be followed by prolonged contraction of the extensor muscles and still more prolonged relaxation.

Make a gastrocnemius muscle preparation. Fasten the muscle to a muscle lever and bring the writing point against a smoked drum. Record a single contraction.

Note the increased height of the phase of shortening, and the prodigious increase in the duration of the plase of relaxation. This contracture (page 340 ) is lessened by repeated stimulation, but reappears if the muscle be allowed to rest. Cooling or warming usually causes the veratrine effect to disappear temporarily.

A quick initial contraction may precede the characteristic veratrine contraction, possibly because the veratrine affects differently the red and the clear fibres. 


\section{Tetanus}

Superposition of Two Contractions.- Arrange a gastrocnemius muscle to write on a smoked drum. Connect the binding posts on the muscle lever and muscle clamp with the secondary coil of an inductorium. In the primary circuit (posts 1 and 2) place the electro-magnetic signal and a simple key. Let the drum revolve at a rapid rate. Send two maximal induction currents through the muscle at varying intervals, beginning with the shortest interval possible. The secondary should be at such a distance from the primary coil that both make and break currents shall cause maximal contraction.

If the second stimulus fall in the latent period of the first contraction, the stimulus will be without effect. If the second stimulus fall between the beginning of shortening and the end of relaxation caused by the first stimulus, the contraction following the second stimulus will not begin from the base line, but will be superposed on the first, as if the state of shortening from which the second contraction begins were the resting stage of the muscle. The height reached by the second contraction will be greater than that reached by the first. The summed height is 
usually greatest when the second contraction starts from the summit of the first, but this rule is not invariable. The summit of the summed contraction does not necessarily coincide with the summit of the second contraction; the higher the summed contraction, the quicker the summit is reached.

Superposition in Tetanus. - Place the vibrating interrupter (Fig. 50) in the primary circuit. Repeat the preceding experiment, but use a series of stimuli instead of only two. It will be observed that a third contraction may be superposed on the second, a fourth on the third, and so on. The shortening of muscle, however, has a limit; and when this is reached, further stimulation merely maintains this maximum degree of shortening until fatigue sets in. When the interval between successive stimuli is very brief the successive contractions appear to fuse together and the contraction curve becomes a continuous line. The more rapid the contraction, the shorter must be the interval between successive stimuli in order to cause the disappearance of the individual contractions. Thus a more rapid rate of stimulation is necessary to produce complete fusion in fresh, highly irritable muscles than in those the irritability of which has been diminished by cold or fatigue. For this reason 
contractions which at the beginning of the stimulation period are marked by notches in the curve fuse completely as longer stimulation brings on fatigue. Here also the differences in the structure of muscles already mentioned play an important part. Thus the red muscles of the rabbit are thrown into tetanus by a much smaller number of stimuli per second than are the more quickly contracting white muscles.

Relation of Shortening in a Single Contraction to Shortening in Tetanus. - 1. Record side by side the contractions of a muscle unloaded except by the muscle lever. Stimulate with a single maximal induction current; stimulate with a brief tetanizing current.

The shortening of the single twitch of the unloaded muscle is as great as the shortening in tetanus.

2. Load the muscle with ten grams and repeat Experiment 1.

The shortening in tetanus will now be considerably greater than that of the single twitch.

3. Load the muscle with ten grams but support the weight by the after-loading screw, so that the weight cannot pull on the muscle until the contraction begins. Record one contraction on a stationary drum in response to a maximal make induction current. 'Turn the drum one 
millimetre. Raise the writing point of the lever one millimetre by means of the after-loading screw. Stimulate the muscle with a make induction current of the same intensity as before. Again turn the drum and raise the point of the lever one millimetre, and stimulate the muscle as before. Continue this until the after-loading screw is raised so high that the muscle no longer shortens sufficiently to raise the lever.

Obviously in this experiment the weight is artificially supported during a progressively greater portion of the contraction. It will be found that the total shortening of the muscle loaded only during the latter portion of the contraction is as great as the shortening of a loaded muscle in tetanus.

\section{The Isometric Method}

Thus far we have observed the development of energy in a muscle stretched by a small, unvarying load. The principal part of the energy set free in this isotonic process appears as the mechanical energy of a visible change in form; a small part of the energy of the muscle is converted into tension. Fick has pointed out that if the muscle be made to pull against a strong spring, the change in the length of the muscle 
will be very slight, and the greater portion of the energy will be converted into tension and stored in the spring. If the excursion of the spring be recorded by a writing lever, the curve will be practically a record of the course of transformation of energy into tension, and will be only to a slight extent the record of a change in form.

In order to determine the amount of energy converted into tension in the isometric contraction, it is necessary to graduate the spring against which the muscle pulls.

Graduation of Isometric spring. - Attach the large scale-pan to the strong spring of the apparatus shown in Fig. 60. Place a long straw on the end of the spring. Bring the writing point against the smoked paper of a kymograph. Turn the drum once round to record an abscissa. Return the drum to its former position, and place 80 grams in the scale-pan attached to the spring. When the spring is stretched tirn the drum once round to record the bending under 100 grams' weight. ${ }^{l}$ Restore the drum to its former position, add 100 grams, and make record of the extension at 200 grams. Continue the record up to 1000 grams. Preserve the curve for reference (page 363).

1 The scale-pan weighs about 20 grams. 
The Heavy Muscle Lever. ${ }^{1}$ - It is sometimes necessary to after-load a muscle lever with weights far in excess of those that a light muscle lever will bear without "springing" and thus altering the abscissa. Such heavy loads are borne by the heavy muscle lever illustrated in Fig. 60. A tripod of japanned malleable iron, 27 $\mathrm{cm}$. high and $17.5 \mathrm{~cm}$. broad at the base, supports a femur clamp and a muscle lever. The latter is a steel tube $5 \mathrm{~cm}$. long, pierced by a steel axle $9 \mathrm{~mm}$. long, revolving between heavy brass posts. The lever weighs about 2.5 gms. The aluminium scale-pan weighs about 20 gms. ; it holds one hundred 10-gram weights. The lever may be turned completely over in a backward direction, and

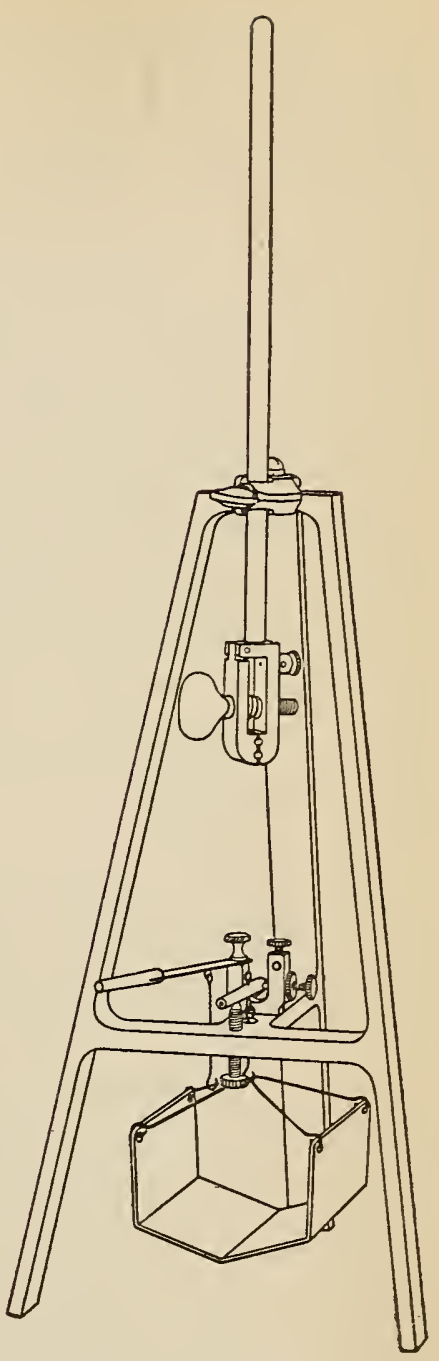

Fig. 60. The heary muscle lever.

1 American Journal of Physiology, 1903, viii, p. xl. 
thus be entirely out of the way. The steel spring shown upon the left of Fig. 60 may then be turned to the right to bring its wire hook into the opening through which the scale-pan is reached. The scalepan may then be attached to this isometric spring and the spring empirically graduated. When the graduation scale has been written, the milled screw that holds the isometric spring upon the left-hand post (Fig. 60) may be loosened, the spring turned with the hook up, and the screw made fast again. The lower end of the muscle may now be attached to the hook upon the spring and an isometric curve written.

The screw clamp holding the muscle clamp is insulated. A binding post upon the muscle clamp, and another binding post upon the right-hand post supporting the axle of the lever, allow direct stimulation of the muscle.

This lever serves especially well for the double abductor preparation of Fick, consisting of the semimembranosus and gracilis of both sides.

Isometric Contraction. - Invert the spring. Fasten the femur of a gastrocnemius preparation in the muscle clamp, and the Achilles tendon to the spring. Connect the binding posts on the lever and the clamp with the secondary coil of the inductorium, arranged for single maximal induction currents. Let the drum revolve at a rapid speed. Stimulate the muscle with a maximal break current. 
An isometric contraction will be recorded.

Remove the spring, and attach the tendon to the lever weighted with ten grams. Stimulate the muscle with a break induction current of the strength used before.

The usual isotomic curve will be written. Comparison of the isometric and isotonic curves reveals as a rule in the isometric curve a longer phase of rising energy and a flattened summit or plateau. The muscle reaches its maximum tension sooner than its maximum shortening and maintains the maximum tension longer than the maximum shortening.

\section{Contraction of Human Muscle}

Simple Contraction or Twitch. - Place the middle, ring, and little fingers in the support of the ergograph (Fig. 61). Let the adjustable rod rest on the index finger near the distal end of the middle phalanx. Place the point of the rod in the hole nearest the free end of the spring. Adjust the writing point to write on a smoked drum revolving at moderate speed. With the brass electrodes covered with wet cotton (page 129), stimulate the abductor indicis with a single maximal break induction current. Compare the form of the curve thus obtained with the contraction curve of the skeletal muscle of the frog. 
The Ergograph. ${ }^{1}$ - A flat, steel spring provided with a writing point is fastened in a stont iron support clamped to the table (Fig. 61). The second, third, and fourth fingers of the subject's hand are fastened with tapes to the wooden support. Upon the index finger

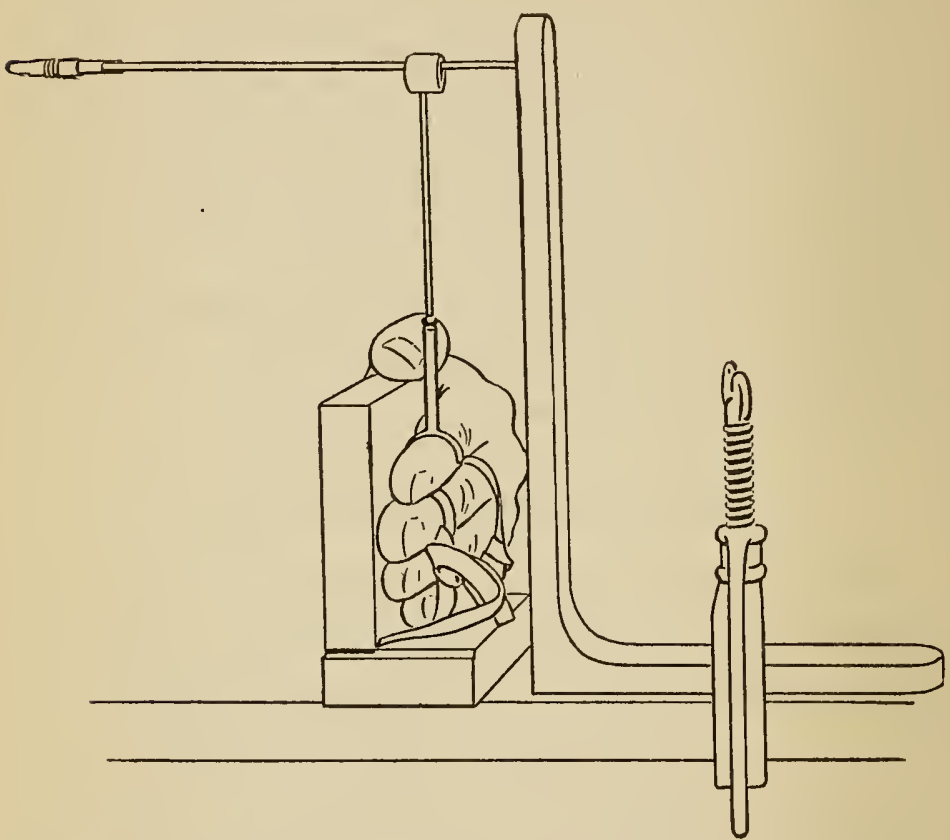

Fug. 61. The ergograph; also employed for recording the isometric and isotonic contractions of human muscle.

near the distal end of the middle phalanx is placed a rod the length of which is adjustable. The point of the rod passes through a rubber ring and presses against the under sille of the spring. When the point

1 First describerl in the "Introduction to Physiology," 1901, p. 220. 
is near the free end of the spring, contractions of the abductor indicis muscle with voluntary and electrical stimuli may be recurded. When the point of the rod is placed near the cast-irnn support of the spring, the movements of the spring will be so much less that almost none of the energy of the muscle will be converted into mechanical motion. An isometric contraction will be recorded.

Isometric Contraction. - Place the point of the adjustable rod in the hole nearest the cast-iron support of the spring. The movement of the spring is so much less at this point that almost none of the energy of the muscle will be converted into mechanical motion. Stimulate the muscle as before with a maximal break induction current. Compare the isometric curve thus recorded with the largely isotonic curve previously obtained.

Artificial Tetanus. - Replace the adjustable rod in its former position (isotonic arrangement). Stimulate the abductor with the tetanizing current of the inductorium. Compare the curve with the tetanus of frog muscle.

Natural Tetanus. - 1. Contract the abductor by voluntary impulse. This also gives a tetanus curve. When the natural tetanus is prolonged, it frequently is marked by oscillations having a periodicity of about ten per second. 
2. Place the adjustable rod in the hole nearest the iron support (isometric arrangement). Stimulate the muscle (1) with the tetanizing current of the inductorium; (2) by voluntary impulse.

It will be seen that the energy set free by the natural stimulus is much greater than when the muscle is stimulated artificially.

\section{Smooth Muscle}

Spontaneous Contractions. - Make two cuts, $5 \mathrm{~mm}$. apart, through the frog's stomach at right angles to the long axis. Pass a bent hook through the ring (i.e. through the cavity of the stomach), and fasten the hook in the muscle clamp. Pass a second hook around the lower margin of the ring and attach it by means of a fine copper wire to the straw of the heart lever (Fig. 53). Contraction of the circular fibres. can thus be made visible. Bring the writing point against a drum revolving about once an hour. Wrap filter paper saturated with normal saline solution about the muscle ring. Keep this thoroughly moist. Proceed to the remaining experiments, observing the stomach preparation from time to time.

Spontaneous rhythmic contractions will appear. Note the changes in tonus. 
Simple Contraction. - Prepare a second ring of frog's stomach in the manner described in the preceding experiment. Attach the lower margin of the ring to the muscle lever by means of a fine copper wire. Carry the end of the copper wire to the binding post on the muscle lever. Connect this post and the post on the muscle clamp with a dry cell, interposing a simple key. Place the electro-magnetic signal in the primary circuit. Bring the writing points of the muscle lever and the signal against a smoked drum in the same verticle line. Let the drum move at slow speed. Stimulate the muscle by making and breaking the galvanic current once, not oftener.

Compare the duration of the latent period with that of skeletal muscle. Compare the form of the contraction curve with that of skeletal muscle.

Tetanus. - Determine how frequent the stimuli must be in order that the separate contractions may be fused into a smooth curve.

Usually the muscle after contracting loses its irritability for several minutes. If this occur, the ring may be laid aside, covered with filter paper saturated with normal saline solution. Excellent curves are often obtained from muscle preserved in this way for half an hour or more. 


\section{The Work Done}

Influence of Load on Work done. - In the tracings obtained in the experiments on page 341 with loads of 10 grams and upwards measure the distance from the summit of each curve to the abscissa. Calculate the gram-millimetres of work done at $10,30,50,70$, and 90 grams, using the formula $W=\frac{w h}{m}$ in which $W$ is work done, in gram-millimetres; $w$, the weight lifted in grams, $-i$. $e$. the weight of the scale-pan and lever (about 12 grams) plus the weight put into the scale-pan (the weight of the muscle itself may be neglected); $h$, the height, in millimetres, to which the load is lifted; $m$, the magnification of the lever.

Write the results on the smoked paper.

Note that within wide limits an increase in the load increases the work done by the muscle.

Absolute Force of Muscle. - Secure the femur of a gastrocnemius muscle preparation in a muscle clamp and fasten the tendon to the rigid muscle lever. After-load the muscle until it just fails to lift the load when stimulated with tetanizing induction currents.

The load which neither extends a contracting muscle nor allows it to shorten is a measure of the "absolute force" of the muscle. 
Total Work done; the Work Adder. - Attach a scale-pan to the cord that passes over the pulley on the axle of the work adder (Fig. 62).

The Work Adder. ${ }^{1}-\mathrm{A}$ wheel of aluminium (Fig. 62) bears upon its axle a counterpoised muscle lever ending in a pawl through which the wheel is caused to revolve when the lever is pulled upward by the attached muscle. A second pawl prevents the wheel from turning back when the muscle relaxes. The axle of the aluminium wheel bears on the other side a pulley, from which the weight is suspended. The turning of the wheel winds the suspending cord upon the pulley and thus raises the weight.

Clamp the work adder to a stand in such a way that

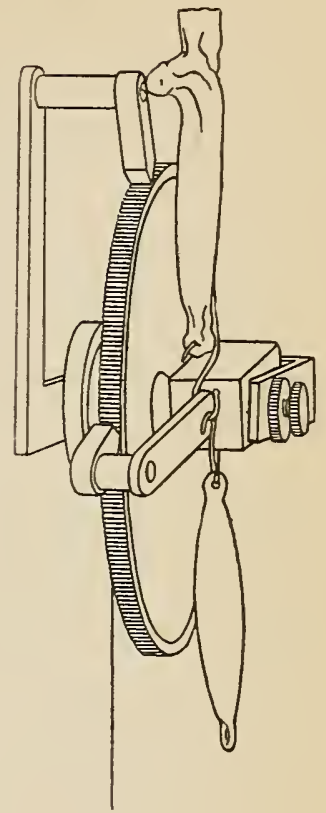

Fig. 62. The work adder; about six-sevenths the original size. The handle is not shown. The muscle preparation is the double abductor suggested by Fick.

the scale-pan hangs free of the table. Fasten the tendon of the gastrocnemius muscle preparation

1 Introduction to Physiology, 1901, p. 225. The first work adder was devised by Fick. 
to the lever of the work adder at a distance from the axis of the pulley equal to the radius of the pulley. Connect the muscle with the secondary coil of an inductorium arranged for single maximal induction currents. Measure the distance of the pulley weight from the level of the axis of the pulley. Stimulate the muscle with induction currents at intervals of one second until the fatigued muscle ceases to contract. (Stimulation may be made by opening and closing a simple key in the primary circuit in unison with the beat of a metronome.)

Measure the height in millimetres to which the pulley weight has been lifted. Multiply this height by the weight. The product is the total work done in gram-millimetres.

Total Work done estimated by Muscle Curve. The total work done by the muscle may also be estimated by measuring in millimetres the height of each successive contraction recorded on the smoked paper, adding the several heights together, dividing the sum by the number of times the distance from the fulcrum of the recording lever to the point of attachment of the muscle is contained in the distance from the fulcrum to the writing point, and multiplying this quotient by the sum of the pulley weight plus the weight of the lever. 
In tetanus no weight is raised and no visible mechanical work is performed. That internal work is performed is shown by the rise in temperature.

Time Relations of Developing Energy. - The simple muscle curve is a graphic record of the mechanical energy set free by the muscle in lifting a certain load. It is desirable to measure the maximum energy that the muscle can set free at each moment from the beginning of contraction to the point at which the greatest shortening is reached.

Place the electromagnetic signal in the primary circuit of an inductorium arranged for maximal make induction currents. Arrange a tuning fork to write on a smoked drum beneath the line drawn by the writing point of the signal. Fasten the femur of a gastrocnemius muscle in the muscle clamp and attach the tendon to the heavy muscle lever. Place the three writing points in the same vertical line. Connect the binding posts on the muscle clamp and the lever with the posts of the secondary coil of the inductorium. "Afterload " the muscle with 50 grams. Set the tuning fork vibrating. Spin the drum. Stimulate the muscle with a single maximal make induction current.

The muscle will not shorten until the energy 
set free is sufficient to lift a load of 50 grams. Turn the drum until the writing point of the signal rests in the line made by the signal when the muscle was stimulated. Let the drum be stationary. Set the tuning fork vibrating. Its writing point will mark a line synchronous with that drawn by the signal during the experiment. Revolve the drum a little farther, until the writing point of the muscle lever reaches the point at which contraction began. Set the tuning fork vibrating again. Its writing point will mark a line synchronous with the beginning of contraction. The number of vibrations in the tuning fork curve between the two points just recorded is the interval between the stimulation of the muscle and the point at which the energy set free was sufficient to move a load of 50 grams. Note this interval.

After-load the muscle with 100,150, 200, 250, and 300 grams, and repeat the above experiment after each addition of 50 grams.

On coordinate paper set down as ordinates the several loads employed and along the abscissa the time intervals in hundredths of a second. Place a dot at the junction of the 50-gram line with the perpendicular cutting the abscissi at the figure indicating the interval observed between stimulation and the moment when the energy 
developed sufficed to raise the load. Repeat this with other loads. Join the dots. The resulting line is a curve showing the absolute force of the muscle at successive intervals from the beginning to the end of the phase of rising energy.

Record with this same muscle an isometric contraction (page 350). With the aid of the graduation scale of the isometric spring ascertain the maximum tension developed in the isometric contraction. Compare this result with that secured in the experiment just concluded on the time relations of developing energy.

\section{ELasticity and Extensibility}

Elasticity and Extensibility of a Metal Spring. Clamp the ergograph (Fig. 61) to the table in such a way that the writing point of the ergograph spring shall rest against a smoked drum. Attach a scale-pan to the spring near the free end. Turn the drum once round by hand, thus describing an abscissa on the smoked paper. With the forceps place 2 ten-gram weights very carefully on the scale-pan.

The spring extends. Turn the drum $2 \mathrm{~mm}$. and add another 20 grams to the scale-pan.

A further extension of the spring will be recorded. 
Turn the drum $2 \mathrm{~mm}$. again. Continue to record the extension of the spring after each addition of 20 grams until a load of 200 grams has been reached.

It will be found that the extension eurve is a straight line. The extension is directly proportional to the weights employed.

Remove the weights 20 grams at a time, turning the drum $2 \mathrm{~mm}$. after each lightening.

The spring will return to its former length. Its elasticity (within the limits of extension here used) is perfect.

Of a Rubber Band. - Place the muscle clamp in the stand of the heavy muscle lever (Fig. 60). Seeure a rubber band in the jaws of the elamp and fasten the other end of the band to the musele lever. Repeat the preceding experiment, using 10-gram loads instead of 20 -gram loads.

The extension curve will again be a straight line. The return to the original length will not be complete. The elasticity of the rubber band is not perfect. An "extension remainder" is present. After a considerable time the extension remainder will disappear and the band will return to its former length, provided the extension was not too violent nor too long-continued.

Of Skeletal Muscle. - Isolate in both limbs the 
mass of long, parallel-fibred muscles extending along the inner side of the thigh from the pelvis to the tibia. Separate from the remainder of the pelvis the portion to which the muscles of both sides are attached. Remove the muscles of both sides together with the part of the tibia and the pelvis in which they are inserted. The muscles of the two sides thus form practically one long muscle held together in the middle by the small piece of bone into which they both are inserted (Fick's preparation, Fig. 60).

Repeat the preceding experiment, using this preparation in place of the rubber band.

The extension curve is no longer a straight line, but approximately a parabola. In organic bodies, the increase in length is not proportional to the extending weights, but grows smaller as the weight increases.

A perfectly fresh muscle weighted lightly (e.g. 10 grams) usually returns to its original length when the extending weight is removed. With larger weights, the return is not at first complete: an extension remainder is observed, and the original length is reached only after a considerable time.

Extensibility increased in Tetanus. - With the gastrocnemius muscle (unloaded except by the writing lever and scale-pan) draw an abscissa (1) 
with the muscle at rest; (2) with the muscle tetanized. These abscissie record the length of the practically unloaded muscle in the resting and the active states. Place 10 grams in the scale-pan and again record the length of the muscle (1) at rest; (2) tetanized. Make similar records for each 10 grams up to 100 .

It will be found that the extension curve falls more rapidly in the active than in the resting muscle; the extensibility is increased in tetanus.

\section{Fatigue}

Skeletal Muscle of Frog. - 1. Let a gastrocnemius muscle loaded with 10 grams write its contractions on a very slowly moving drum. Connect the secondary coil with the binding posts on the muscle clamp and the muscle lever. Stimulate the muscle once in two seconds with a maximal induction current, using make and break currents alternately. The correct interval may be obtained by listening to the beat of a metronome. Continue to record the contractions until the muscle will no longer shorten when stimulated (exhaustion).

State the characteristic features of the fatigue curve.

2. With a fresh muscle repeat the stimulation 
every two seconds until the height of contraction has diminished about one half. Now record the duration of the latent period, phase of rising energy, and phase of sinking energy (page 334) on a rapidly moving drum.

Note the absolute and relative duration of these periods as compared with those of muscle not fatigued.

3. Stimulate a sartorius from the same frog continuously with tetanizing currents and record the tetanus curve.

State the differences between the fatigue curve thus secured and the curve obtained by less frequent stimulation.

Attention has already been called to the differences which depend on the relative proportion of red and clear fibres (page 336). The latter are more easily fatigued.

Human Skeletal Muscle. - 1. Arrange the ergograph to record the contractions of the abductor indicis, as directed on page 353. Place the point of the adjustable rod in the hole nearest the free end of the spring.

Prepare also the large and small brass electrodes for artificial stimulation of the muscle and place them in position.

Bring the writing point against a very slowly moving drum. Contract the muscle voluntarily 
twice every second, keeping time with the beat of a metronome, until two hundred contractions have been made.

Now stimulate artificially every two seconds, using maximal make and break currents alternately, until two hundred contractions have been made.

State the characteristics of the two fatigue curves, and compare the curves with those obtained from frog's skeletal muscle.

2. From a fresh subject obtain a fatigue curve by artificial stimulation of the abductor indicis, using maximal make and break induction currents alternately every two seconds, as directed in the preceding experiment. When the muscle has been stimulated two hundred times, contract it voluntarily every two seconds until two hundred contractions have been made.

Compare the curves with those obtained in Experiment 1.

Explain these paradoxes.

It has been pointed out on page 357 that smooth muscle loses its irritability much more rapidly than striated muscle.

\section{Apraratus}

Normal saline. Bowl. Towel. Pipette. Glass plate. Volume tube. Bunsen burner. Inductorium. 'Two dry 
cells. Wires. Muscle clamp. Fine copper wire. One hundred ten-gram weights. Musele lever. Electro-magnetic signal. Kymograph. Tuning fork. Cork clamp. Four needle electrodes. Pole-changer. Pin. Cork. Two stands with clamps. Ten one-gram weights. Musclewarmer. Split shot. Ice. One per cent solution of veratrine acetate. Wheel-interrupter. Vibrating reed. Straw $36 \mathrm{~cm}$. long with platinum contact. Mercury cup. Rigid muscle lever. Spring ergograph with rod. Hand clamp. Ergograph clamp. Large weight pan. Cotton. Two bent hooks. Heart-holder. Filter paper. Simple key. Work adder. Co-ordinate paper. Rubber band. Metronome. 


\section{IV}

THE CENTRAL NERVOUS SYSTEM

\section{Simple Reflex Actions}

The Spinal Cord a Seat of Simple Reflexes. 1. By means of a hook or thread passed through the lower jaw suspend vertically a frog the brain of which has been destroyed with the seeker; the legs must not touch the table. Pinch a toe with the forceps.

The leg will be drawn up.

A stimulus to the skin has caused the contraction of muscles. The afferent impulse set going by the sensory stimulus is changed into a motor efferent impulse. This is an example of reflex action.

2. Destroy the spinal cord with the seeker. Stimulate the skin of the right leg electrically and mechanically.

In no case will the sensory stimulus call forth the reflex contraction of a skeletal muscle. Yet the nerves coming from the skin and going 
to the muscles are still intact. Only the spinal cord has been destroyed.

The conversion of sensory into motor impu'ses for skeletal muscles is a function of the central nervous system.

\section{Influence of Afferent Impulses on Reflex Action.-}

Destroy the brain of a strong frog with the seeker. Gently pinch a toe of the right foot.

Only the right leg will be drawn up.

Pinch a toe of the left foot.

Only the left foot will be drawn up.

Pinch a finger.

Only the corresponding arm will move.

Pinch the whole foot sharply.

More extended movements will be made.

The character and location of the stimulus affect the resulting contraction.

Threshold Value Lower in End Organ than in Nerve-Trunk.-1. Carefully expose the sciatic nerve. Determine the least strength of tetanizing current that will cause a crossed reflex when applied to the skin of the foot. Now apply the same stimulus to the trunk of the nerve.

As a rule, the intensity required to produce reflex action is less when the stimulus is applied to the peripheral endings of the sensory nerves than when the nerve-trunks are stimulated. 
2. Divide the skin over the back in the median line. Raise the skin on one side until the small nerves which pass across the dorsal lymph sac to innervate the skin come into view. Sever from the surrounding skin a piece about one centimetre square containing the endings of one of the nerves. Let the isolated piece with its nerve endings remain connected with the body only by the trunk of the nerve. As before, determine the least strength of tetanizing current that will cause a reflex movement when applied to the nerve-endings in the skin and to the nerve-trunk respectively.

The threshold value for reflex action will again be found lower in the nerve-endings than in the nerve-trunk.

Summation of Afferent Impulses. - Pass two fine copper wires about the frog's foot a centimetre apart and connect them with the secondary coil. Connect the primary coil through a simple key with a dry cell. Stimulate with regularly repeated make induction currents of such strength that single stimuli cause no reflex contraction.

Summation of the subminimal stimuli will finally cause reflex contraction.

Determine that the number of stimuli necessary to produce a reflex becomes smaller when (1) 
the strength of the induction currents is increased, and (2) when the interval between the stimuli is lessened.

Segmental Arrangement of Reflex Apparatus. 1. Gently pass the seeker over the abdominal walls on one side.

The muscles in that region only will twitch.

Repeat the stimulus, but use a stronger pressure.

The area contracting will increase in extent approximately in proportion to the increase in the stimulus. The afferent nerves from any one region are more closely related to the efferent nerves of that same region than to those of other regions. The fact that both afferent and efferent fibres spring from the cord at the same level suggests that their nerve cells lie also at approximately the same level. On increasing the stimulus the afferent impulse spreads from segment to segment of the cord. Further evidence of the segmental arrangement will be gained by the following experiment.

2. With a clean, sharp knife make transverse sections of the spinal cord, beginning in the cervical region. A short time after each section test the reflexes from the hind limb by mechanical stimulation. 
Note the level below which no section can be made without rendering the reflex impossible. The nerve cells concerned in this reflex lie on the caudal side of this line.

Now in a second frog make transverse sections, beginning at the caudal end of the cord, and test the reflexes as before, until the level is reached beyond which a section will destroy the reflex.

Observe that the portion of the cord comprised between the two levels determined forms a seginent which contains the central apjaratus concerned in the reflex studied.

Reflexes in Man. - 1. From the Slin. - Rub the plantar surface of the foot gently with some hard object.

The foot will be retracted reflexly.

Similar results may be obtained by rubbing the skin of the inside of the thigh, which will cause contraction of the cremaster muscles; or by rubbing the skin of the abdomen, which will be followed by contraction of the abdominal muscles.

These reflexes are of importance in clinical diagnosis becanse by means of them the seat of a discased area in the central nervous system may sometimes be defined, since the reflex depends on the integrity of the corresponding reflex are.

2. Cornea lirflex. - Touch the cornea gently with a thread. 
The eye will be closed involuntarily.

3. Throat Reflex. - Touch the posterior wall of the throat.

The movements of swallowing will usually follow.

4. Pupil Reflexes; Light Reflex. - Close one eye for several seconds, then open it quickly.

Note the contraction of the pupil.

5. Consensual Reflex. - Close one eye as before, but watch the pupil of the other eye when the first is opened again.

The pupil will contract.

6. Accommodation Reflexes. - Look alternately at a near and a far object. The pupil will contract when the eye adjusts itself to see the near object.

\section{Tendon Reflexes}

Knee Jerk. - Sit in such a position that the knee is bent at a right angle, and the foot hangs free. Let an assistant strike the patellar ligament with the side of the hand.

Note the sudden contraction of the extensors of the thigh, the so-called knee jerk.

Flex the knee at different angles and determine in which position the resulting contraction is greatest.

Knee jerk can be obtained only within certain limits of extension. 
Let the subject immediately before the stimulus is applied forcibly contract some other group of muscles; clench the hand, for example.

The knee jerk is reinforced.

Ankle Jerk. - Bend the foot at right angles to the leg, and strike the tendo Achillis. The experimenter should hold the end of the foot in his left hand.

Contraction of the gastrocnemius muscle will be observed.

Gower's Experiment. - Strike the side of the tendo Achillis.

A contraction will result.

Support the other side of the tendon so that the gastrocnemius muscle will not be stretched by the blow. Repeat the experiment.

No contraction follows. The tendon jerk requires for its production a rapid stretching of the muscles involved in the contraction.

Try to obtain tendon jerks from other muscles; for example, the triceps humeri, flexors of hand, and masseter muscles.

Normally no response will be obtained.

The experiments are of value in diagnosis of aiseases of the central nervous system. 
Effect of Strichnine on Reflex Action

Inject with a glass pipette a few drops of 0.5 per cent solution of sulphate of strychnine into the dorsal lymph sac of a frog the brain of which has been destroyed with a seeker.

After a few minutes, very weak afferent impulses will be sufficient to call forth general spasmodic reflex actions. Note that (1) the strychnine reflexes are paroxysmal, (2) the muscles fall into more or less prolonged rigidity (tetanus), and (3) the extensors overcome the flexors, the limbs being strongly extended.

The characteristic action of strychnine is evidently not dependent on the brain.

Destroy the spinal cord with a seeker.

Stimulation of muscles and nerves will not cause spasmodic contractions.

Strychnine acts on the spinal cord, but not on the muscles or the peripheral nerves.

\section{Complex Co-ordinated Reflexes}

Removal of Cerebral Hemispheres. - Place a frog under a glass jar containing a small sponge wet with ether. Be very careful not to kill the frog. When insensibility is complete, place the animal on a frog-board. Cut through the skin in the median line of the skull, from the nose to the 
vertebral column. Connect the front margins of the two tympanic membranes by a transverse incision through the skin. This transverse line will pass over the junction of the cerebral lobes with the optic lobes. Strip off the parietal bones with forceps, beginning at the anterior end opposite the anterior margin of the orbit. When the cerebral hemispheres are uncovered, they may be removed from before backwards. Avoid injuring the optic lobes. Work rapidly but carefully. If the ether effect diminish before the operation be finished, replace the frog under the glass jar for a few moments. As soon as the hemispheres are removed, sew up the wounds in the skin.

Note the signs of profound inhibition.

If the operation be done carefully, the shock will gradually pass away, and the functions possible in the absence of the cerebrum may then be determined. Put the frog aside, moistening his skin occasionally, but not otherwise disturbing him, and prepare a second frog for the experiinent upon the "croak reflex" (page 379). When this operation is completed, resume the observations on the first frog, while the second frog recovers from the shock.

1. Posture, etc. - Write down the differences between the fing from which only the cerebral hemispheres have been removed and a frog in 
which the whole brain has been destroyed with the seeker, in respect to posture, power to regain feet when laid on back, respiratory movements, position of eyelids, leaping and swimming.

2. Balancing Experiment. - Place the frog on a somewhat roughened board, about 20 inches long, 8 inches wide, and 1 inch thick. Tilt the board gradually.

The frog remains motionless until his centre of gravity is disturbed. He then moves forward in an attempt to reach a stable position. By careful management, he can be made to climb up the inclined board, perch upon the narrow edge, and, the board still turning, descend head-first on the opposite side.

3. Retinal Reflex. - Place the frog deprived of cerebral hemispheres in front of a bright light; for example, an incandescent electric lamp. Interpose some object, such as a small instrument case, between the light and the frog, so that a strong shadow is cast upon the frog's eyes. Stimulate the frog by pinching the skin of the back.

The frog will jump, but will avoid the object which casts the shadow.

4. Croak Reflex. - Sever the large hemispheres from the remainder of the brain of another frog by passing a knife through the cranium to the 
base of the skull from side to side in a line joining the anterior margins of the tympanic membranes. (Where possible, a male frog should be selected for this experiment. Males may be recognized by the cushion-like thickening on. the innermost digit of the manus, or hand; the male Rana esculenta possesses bladder-like, resonating pouches connected on each side with the mouth cavity.) After the immediate shock of the operation has passed, stroke the back over the anterior half of the spinal cord.

Reflex croaking will be observed.

The croak reflex can be inhibited by simultaneous pinching of one of the limbs or other strong stimulation. (Compare page 384.)

If the experiments on the frog in which the cerebral hemispheres were extirpated were not satisfactory, repeat them on this frog in which the hemispheres were simply separated from the remainder of the brain.

These observations teach that very complicated co-ordinated actions are possible in the absence of the large hemispheres. Only simple reflexes are possible when the whole brain is removed. Consequently, the seat of these complicated reflexes must lie in the brain between the cord and the cerebral hemispheres. 


\section{Apparent Purpose in Reflex Action}

1. Destroy the brain of a frog with the seeker. Dip small pieces of filter paper in strong acetic acid. Remove the superfluous acid, lay the paper bearing the acid on (1) the frog's thigh, (2) the foot, (3) the back. After each stimulation note the character of the reflex movement, and then carefully wash the acid from the skin.

The movements are related to the areas stimulated in a certain purposeful way. Efforts are made apparently to brush away the acid paper.

2. Place the acid on the flank of the right leg. Usually the leg stimulated strives to brush away the paper. Hold this leg fast.

The other leg (the left) will be used to remove the acid from the opposite limb. (This experiment succeeds best in strong, lively frogs.)

3. Place an uninjured frog in an evaporating basin containing sufficient water to immerse the frog to the neck and covered with wire gauze to keep him from jumping out. Warm the water.

As the temperature rises to from $20^{\circ}-30^{\circ} \mathrm{C}$. the frog will attempt to escape.

Repeat the experiment with the frog the brain of which has been destroyed.

No movements of escape will be noticed. 
About $35^{\circ}$, muscular twitchings will be seen. At $38^{\circ}-40^{\circ}$ death takes place and the muscles become rigid (heat rigor).

This observation shows that volition in all probability is absent in the brainless frog. It follows that reflex actions are not volitional; their "purpose" is only apparent.

\section{Reflex and Reaction Time}

Reflex Time. - Destroy the brain of a frog with the seeker. Hold one leg of the frog aside with the glass rod. Bring beneath the other a small beaker almost full of dilute sulphuric acid (2:1000). Raise the beaker until the foot is immersed to the ankle. Count the seconds between the application of the stimulus (sulphuric acid) and the withdrawal of the foot.

This interval is the reflex time.

Wash the foot carefully in the bowl of water.

Reaction Time. - Smoke a drum. Raise the drum off its friction bearing by turning the screw at the top of the shaft. Place the writing point of an electromagnetic signal against the smoked paper. Arrange a tuning fork to write its curve near that of the signal. Connect the signal through two simple keys and a dry cell with the primary coil of an inductorium arranged for maximal single induction currents (posts 1 and 
2). Let stimulating electrodes pass from the secondary coil (bridge up) to the tongue of the subject. Let the subject hold one key closed until he feels the stimulus on the tongue.

Direct the subject to shut his eyes. Let the observer start the tuning fork, spin the drum, and stimulate the subject by completing the primary circuit. The instant the subject perceives the stimulus, he will break the circuit by releasing his key. By means of the tuning fork curve determine the interval between stimulation and response. This interval is the reaction time plus the errors of observation; for example, the latent period of the electromagnetic signal. Repeat the experiment three times and take the mean of the results.

In the laboratory note-book make a list of the links in the chain between stimulus and response, and state as far as possible the errors of observation.

Reaction Time with Choice. - Connect the side cups of a pole-changer (without cross wires) to the posts of the secondary coil. Connect one pair of end cups with the usual stimulating electrodes, the other pair with large brass electrodes covered with wet cotton. Let the ordinary electrodes touch the foreliead, the other pair the hand of the subject. The other connections should re- 
main as before. Repeat the preceding experiment but tell the subject to signal only when the tongue (or hand) is stimulated. In order to do this he must add to his former reaction a decision as to the part stimulated.

Reaction time with choice is longer than simple reaction time. In general, the more complicated the mental processes involved, the longer will be the reaction time.

\section{INHibition OF RefleXes}

Through Peripheral Afferent Nerves. - Expose the left sciatic nerve for a distance of about 15 $\mathrm{mm}$. in a frog the brain of which has been destroyed. Tie a thread around the distal end, and sever the nerve at the peripheral side of the ligature. Place the central stump of the nerve on the electrodes of the inductorium, the short-circuiting key being closed. Make the primary circuit, and set the hammer vibrating. Now open the short-circuiting key, bring the right foot of the frog into the dilute sulphuric acid up to the ankle, and count the seconds from the moment of immersion to the moment of withdrawal, continuing meanwhile the stimulation of the central end of the left sciatic nerve.

The latent period will be much prolonged.

Wash off the acid carefully. 
Reflex actions may be inhibited by the simultaneous stimulation of sensory nerves.

\section{Through Central Afferent Paths; the Optic} Lobes. - 1. Expose the brain according to the directions already given (page 377). Immediately posterior to the cerebral hemispheres lie the optic lobes, two gray spherical bodies. Separate the cerebral hemispheres from the optic lobes by a transverse incision, and carefully remove the hernispheres. Wait until the shock of the operation has passed. Now suspend the frog so that the tips of the toes hang above a shallow dish containing water made strongly sour to the taste with dilute sulphuric acid. Determine the reflex time. Wash off the acid and, after a moment's rest, sprinkle a very little finely powdered common salt on the cut surface of the optic lobes. Again determine the reflex time.

The reflex time will be found to be markedly increased by the stimulation of the optic lobes.

2. Prepare a second frog in the same manner. Determine the reflex time. Now instead of stimulating the optic lobes, remove them, and again determine the reflex time.

The removal of the optic lobes shortens the reflex time. 


\section{The Roots of Spinal Nerves}

Destroy the brain of a strong, large frog with a seeker. Divide the skin over the vertebral column from the upper end of the urostyle to the level of the fore limbs. Hook back the flaps of skin. Remove the longitudinal muscles on either side of the spines of the vertebræ, thus exposing the bony arches. Saw through the arches of the 8 th, 7 th, and 6th vertebræ (there are ten vertebra in the frog, counting the urostyle) in the order named. Clear away the bone and the underlying tissues until the last three or four pairs of roots shall be plainly seen. Grasp the filum terminale and cantiously lift the cord until the spinal nerve roots are clearly displayed.

The anterior roots are hidden by the large, superficial posterior roots. The conspicuous posterior root which seems to be the last is, in reality, the 9th, the next to the last; the last, or 10 th, is smaller and lies close to the filum terminale. Place a silk ligature about the middle of an anterior and a posterior root on the right side. With single induction currents as stimuli observe that (1) the stimulation of only the central end of the posterior root calls forth a (reflex) movement, and (2) the stimulation of only the peripheral segment of the anterior root causes, movement. 
On this same side cut all the posterior roots.

No stimulus applied to the right leg will now discharge a reflex action. But stimuli applied to sensory nerves elsewhere may still cause reflex movements of the right leg. Motor impulses still pass to these muscles. But only the anterior roots remain.

Hence the anterior roots of spinal nerves transmit motor impulses from the spinal cord towards the muscles (efferent impulses); the posterior roots transmit sensory impulses from sensory surfaces towards the spinal cord (afferent impulses.)

Ludwig's Demonstration. - Destroy the brain of a large frog with the seeker. Remove the thoracic and abdominal viscera, taking care not to injure the sciatic nerve plexus. Remove the 7 th and Sth vertebræ, taking the greatest pains not to injure the nerve roots. Divide the body transversely at this level, so that the anterior and posterior halves shall remain connected only by the anterior and posterior sciatic roots. Keep the roots moist with normal saline solution.

Demonstrate again that the anterior roots transmit efferent, and the posterior roots afferent impulses.

Localization of Movements at Different Levels of the Spinal Cord. - Separate the three roots which form the sciatic nerve. After tying a thread 
about each root sever it from the spinal cord by a cut on the proximal side of the thread. Stimulate each nerve with a very veak tetanizing current. Note the different results obtained from nerves arising at different levels of the cord. Stimulation of the most anterior root causes marked flexion of the limb; stimulation of the middle roots, extension and internal rotation; and of the most posterior, simple extension.

In a frog whose nerves have not been cut expose the spinal cord and stimulate it at different levels in both directions along its length. The various movements of the hind limbs are localized at different levels of the cord.

\section{Distribution of Sensory Spinal Nerves}

Destroy the brain of a large frog with the seeker. Expose the lower half of the spinal cord by the method already described. On one side cut the dorsal sensory root of the Sth spinal nerve and on the other cut the sensory root of the 7 th, 9 th, and 10th. After the section of each root test the cutaneous sensibility of the limbs by placing upon the skin small pieces of filter paper (two mm. square) moistened, not dripping, with 0.2 per cent sulphuric acid. Make a map of the anæsthetic areas in each leg, and note the lack of correspondence. 
Many skin areas are supplied by fibres from at least two sensory roots. The fields of distribution overlap.

\section{Muscular Tonus}

Brondgeest's Experiment. - Fasten a lightly etherized frog back uppermost on the froy-board.

In a line between the ilium and the coccyx open the pelvic cavity by cautiously dividing the skin, fascia, and muscle. Divide the sciatic nerve roots on the operated side. Pass a hook or thread through the jaw and hang the frog up.

Observe that the limb the nerves of which have been cut is relaxed, so that the toes hang lower than those of the limb which still retains its connection with the central nervous system.

\section{Apparatus}

Normal saline. Bowl. Towel. Pipette. Stand. Muscle clamp. Bent hook. Inductorium. Dry cell. Electrodes. Large brass electrodes. Cotton. Key. Frog-board. Fine copper wire. One-half per cent solution of strychnine sulphate. Glass jar with ether and sponge. Balancing board. Strong acetic acid. Filter paper. Evaporating basin. Wire gauze. Bunsen burner. Thermometer. Dilute sulphuric acid (0.2 per cent). Beaker. Kymograph. Electro-magnetic signal. Tuning fork. Pole-changer. Vertebral saw. 


\section{V \\ THE SKIN}

\section{Sensations of Temperature}

Hot and Cold Spots.-With a lead-pencil point carefully explore an area about an inch square on the back of the wrist or hand. Mark with black ink the places where a distinct sensation of cold is felt, and with red ink those where the sensation is one of warmth.

The places indicated are the so-called hot and cold spots.

Outline. - Attempt to define more exactly the outline of one of the cold spots.

The spots are of irregular shape, - blotches rather than points.

Mechanical Stimulation. - 1. Gently tap one end of a small wooden rod the other end of which is placed on a well-defined cold spot.

The mechanical stimulation of the cold spot will give a sensation of cold.

2. Stimulate a warm spot mechanically.

Chemical stimulation. - Rub a menthol pencil over a small area on the back of the hand. 
A sensation of cold will be perceived. This is due to chemical irritation of the cold spots. The temperature of the area does not fall.

Electrical Stimulation. - It has been found that the stimulation of a well-defined cold or warm spot with moderately strong induced currents causes a sensation of cold or warmth respectively.

Temperature After-Sensation.-Stimulate a cold spot mechanically with a pencil point. Remove the point.

The sensation of cold outlasts the stimulus.

Balance between Loss and Gain of Heat. - Provide three beakers of water. Heat them to $20^{\circ}$, $30^{\circ}$, and $40^{\circ} \mathrm{C}$., respectively. Place a finger of one hand in the water at $20^{\circ}$, and a finger of the other hand in the water at $40^{\circ}$. After the respective sensations of cold and warmth have disappeared, place both the fingers in the water at $30^{\circ}$.

The finger from the cold water will seem warm and that from the warm water cold. The temperature of the skin equals the balance between its heat loss and heat gain. When this temperature is raised or lowered, the warm spots or cold spots respectively are stimulated.

Fatigue. - Provide three beakers containing water at $10^{\circ}, 32^{\circ}$, and $45^{\circ}$ C. respectively. Place a finger of one hand in the beaker at $32^{\circ}$, and a 
finger of the other hand in the beaker at $45^{\circ}$. After 45 seconds place both fingers in the water at $10^{\circ}$.

The finger taken from the water at $32^{\circ}$ (which is about the normal temperature of the hand) will feel colder than the other finger. Extreme temperatures of heat or cold fatigue the temperature spots.

Relation of Stimulated Area to Sensation.-Insert a finger of one hand in a beaker of warm or cold water. Note the sensation. Insert a finger of the other hand in the water.

The intensity of the sensation will increase with the extent of the surface stimulated.

Perception of Difference. - Provide two beakers of water, one at $30^{\circ}$, the other slightly warmer or colder. By introducing a finger first into the one and then into the other, and varying the temperature of the water, ascertain how small a difference in temperature can be detected.

Usually a difference of $0.5^{\circ} \mathrm{C}$. is easily recognized.

Relatively Insensitive Regions. - 1 . Compare the temperature sensation perceived on touching with a pencil point the median line of the forehead, nose, and chin with that perceived on touching the skin on either side of the median line. 
The skin in the median line of the body is comparatively insensitive to temperature variations.

2. Similarly compare the mucous membrane with the skin.

The mucous membranes are much less sensitive than the skin.

\section{Sensations of Pressure}

Pressure Spots. - Explore the surface of the forearm by bringing the blunted point of a needle gently in touch with the skin.

At certain spots a distinct sensation of contact will be perceived. Other spots will give only dull sensations. Pressure, like heat and cold, is appreciated by scattered sense-organs in the skin, not by diffuse general sensation.

Note the relation of the pressure points (1) to the hair follicles, and (2) to the warm and cold spots mapped out in previous experiments.

Threshold Value. - Take from the human head several straight, strong hairs. Cement each to the end of a little stick of soft pine to serve as a handle. Provide a special lever, made as follows: With a hot pin burn a small hole at the middle of a straw about $25 \mathrm{~cm}$. in length. Pass a needle through this hole into a cork held in the muscle clamp. Press the free end of the hairs 
against different parts of the skin of the hand, arm, and face. Select hairs which when pressed against the skin of the respective regions give no sensation of pressure. Shorten the hairs until the pressure is just perceptible. This will be the "pressure threshold." Make a loop in a short silk thread and pass the loop about the lever exactly one millimetre from the axis. Hang on the end of the thread a light bent hook. Counterpoise the lever very exactly, so that the slightest force applied to the end of the straw will raise the lever from the after-loading screw. By counterpoising in this way, the lever becomes a balance. On the bent hook hang a ring of German silver wire weighing one decigram (0.1 gram). Find a point on the lever $100 \mathrm{~mm}$. from the axis. The weight of one decigram suspended $1 \mathrm{~mm}$. from the axis of the lever will be raised by a force of $\frac{1}{10} \overline{0}$ of a decigram, equal to one milligram (0.001 gram) applied $100 \mathrm{~mm}$. from the axis. At $50 \mathrm{~mm}$. from the axis, 0.1 gram, suspended $1 \mathrm{~mm}$. from the axis, will be lifted by a force of $\frac{1}{5} \bar{y}$ gram $(0.002$ grami $)$. Find the distance from the axis at which each testing-hair, when pressed vertically against the lever, will just fail to lift the lever; in other words, the point at which the pressure will be just sufficient to bend the hair. The number of millimetres 
between this point and the axis of the lever, multiplied by one-tenth, will give the bending pressure of the hair in the fraction of a gram. Make ten observations on each hair and mark the mean bending value on the wooden handle.

Touch Discrimination. - 1. Close the eyes and let an assistant test the difierent parts of the skin of the hand, arm, and face for discriminating power. For each test separate the points of the resthesiometer until they can be felt as two (ordinary drawing dividers or compasses can be used for an æsthesiometer).

Record your results in millimetres for fingertips, palm of hand, back of fingers, back of hand, back of wrist, flexor and extensor surfaces of forearm, forehead, cheeks, lips, and tongue.

2. Separate the points of the xsthesiometer about $20 \mathrm{~mm}$., and draw them gently side by side along the extensor surface of the forearm from the elbow to the wrist. Repeat the experiment on the flexor surface. Try the same for the cheek and lips, beginning near the ear and drawing the points so that one shall go above and the other below the month.

Describe the sensation in each case, and suggest an explanation.

Weber's Law. - Place the hand palm upward in a comfortable position on the table. Close 
the eyes. Let' an assistant place on the last phalanx of the middle and index fingers a small round box containing ten small shot.

When the subject has formed a clear perception of the weight, let an assistant add or subtract shot, and record the number of shot corresponding to the smallest difference in weight perceived by the subject (whose eyes of course should be kept closed). Repeat the experiment with $20,30,40$, and 50 shot in the box respectively. Determine in each instance the ratio of the number of shot added or subtracted to the number with which each experiment was begun.

This ratio will be approximately constant. The degree of stimulation necessary to cause the perception of difference always bears the same ratio to the degree of stimulation already applied. Weber's law is less true for very small and very large weights than for those of medium value. It is a general law and holds good for visual judgments, etc.

After-Sensation of Pressure. - Place a rubber band about the head and allow it to remain for several minutes.

On removing the band, a distinct after-sensation of pressure will be felt.

Temperature and Pressure. - I'lace on the back of the hand supported on the table a coin the 
temperature of which has been made such that it feels neither warm nor cold. Compare the pressure sensation (apparent weight) of this "normal" coin with that of similar coins warmed and cooled.

The hot or cold coin will seem heavier than the "normal" coin of equal weight.

Touch Illusion; Aristotle's Experiment. - Cross the right middle finger over the right index finger and place them on the palm of the left hand. Place a small shot between the crossed fingers in such a way that it shall touch the ulnar side of the middle finger and the radial side of the index finger. Roll the shot in the palm of the hand.

A sensation of two objects will be felt.

\section{Apparatus}

Black and red ink. Small wooden rod. Menthòl pencil. Inductorium. Dry cell. Electrodes. Key. Three beakers. Stand. Ring. Wire gauze. Bunsen burner. Thernometer. Needle with blunted point. Muscle lever. Gram and ten-gram weights. German silver ring weighing 0.1 gram. Silk thread. Four small wooden handles for pressure-hairs. Bent hook. Drawing dividers (as æsthesiometer). Small round box containing at least 50 shot. Rubber band large enough to go around the head. 


\section{VI \\ GENERAL SENSATIONS \\ TICKLE}

Irradiation. - Gently touch the skin near one nostril with a dry camel's-hair brush.

Note (1) the strong sensation produced by the slight stimulus; (2) the irradiation beyond the spot stimulated.

After Image. - Repeat the stimulus of the preceding experiment.

Measure in seconds the time during which the sensation outlasts the stimulus (after image).

Topography. - Test the tickle sensation at various points on the skin of the face, hands, and forearms. Determine whether the sensation is greatest about the several openings, where skin joins mucous or serous membranes; $c . g$.; the nostrils, the conjunctival sac, the auditory canal. Do the results indicate a protective mechanism?

summation. - In one of the sensitive areas found in the preceding experiment determine the difference between the response to a single stimulus and to successive stimuli.

Fatigue. - In any sensitive area determine (1) the quickness with which the apparatus for the sensation of tickle is fatigued; (2) the duration of fatigue. 


\section{PaIN}

Threshold Value. - Arrange an inductorium for tetanizing currents. Place the electrodes on the tip of the tongue, and move the secondary toward the primary coil until no farther movement can be made without causing the stimulation to become painful. Determine for this region and for others of the mucous membrane of the mouth and of the skin what distance of the secondary coil from the primary separates the stimulus at which pain is just perceived from that at which the pain is distinct.

Latent Period. - In several individuals measure approximately the interval between the application of the stimulus (single break shock) and the resulting painful sensation.

summation. - Determine the number of subminimal stimuli necessary to produce pain.

Topography. - Map upon the skin of the face and arm the areas specially sensitive to pain.

Individual Variation. - Compare the reactions of several individuals, and note the differences in threshold value, latent period, summation, and topography.

Temperature Stimuli. - Fill two bowls or large beakers with water twenty-five degrees respectively, hotter and colder, than the temperature 
of the hand. Determine whether the increase or the corresponding decrease in temperature is the more painful to the immersed hand.

\section{Motor Sensations}

Judgment of Weight. - Lift the same weight twice, at first very slowly and then quickly.

The weight will appear lighter when raised quickly.

Sensation of Effort. - " Hold the finger as if to pull the trigger of a pistol. Think vigorously of bending the finger, but do not bend it.

"An ummistakable feeling of effort results.

"Repeat the experiment, and notice that the breath is involuntarily held, and that there are tensions in other muscles than those that would move the finger." (Sanford.)

Sensation of Motion. - Let the forearm and hand rest upon a table. Bring the four fingers of the hand together, and turn the hand so that it shall rest upright upon the unar side of the little finger. Close the eyes. Abduct the first finger.

The second, third, and fourth finger will seem to move in a direction opposite to the movement of the first. 


\section{VII}

\section{TASTE}

Threshold Value. - Prepare solutions of cane sugar of the following strengths : $1: 1000,1: 800$, $1: 600,1: 400,1: 200,1: 100$. Take half a teaspoonful of the weakest solution into the mouth, roll it upon the tongue, and swallow it. Note whether a sweet taste can be perceived. Rinse the mouth thoroughly. Proceed with solutions of increasing strength until the sweet taste is just perceptible.

Topography. - 1. Select a solution of sugar slightly more concentrated than that just perceived to be sweet. With a small camel's-hair brush apply this solution to the several parts of the tongue and the palate. Determine the regions sensitive to taste. The mouth must be rinsed frequently. 2. Dry the upper surface of the tongue with a handkerchief. With a finely pointed camel's-hair brush apply a twenty per cent sugar solution to the individual fungiform papillie and to the mucous membrane between them. Determine whether only the papilla perceive taste.

Relation of Taste to Area stimulated. - Swallow a very small quantity of a minimal solution of sugar, as determined in the experiment upon 
- threshold value. Rinse the mouth, and then swallow a much larger portion of the solution.

The taste will be perceived more strongly, the larger the area stimulated.

Electrical Stimulation. - 1. Connect two small zinc electrodes through a simple key to a battery of four dry cells. Apply one electrode to an indifferent region, the other to the tongue. Close the key.

Note the sour taste at the positive pole and the alkaline taste at the negative. 
INTRODUCTION TO PHYSIOLOGICAL OPTICS 403.

\section{VIII}

\section{INTRODUCTION TO PHYSIOLOGICAL OPTICS}

ALL visible objects give out light, either of their own making, like the sun, or that which comes from some external source and falls upon their surfaces. Rays that fall upon any surface may disappear (absorption), or be thrown back from the surface (reflection), or, if the body be transparent, pass into it, in which case they are often bent from their course (refraction).

\section{Reflection from Plane Mirrors}

Angles of Incidence and Reflection. - Place in front of the condenser in the lantern (Fig. 63) the diaphragm with $2 \mathrm{~mm}$. aperture. Cover the round window in the optical box with the plain glass slicle. Remove the cork from the tin cylinder. Put a piece of lighted Japanese incense in 


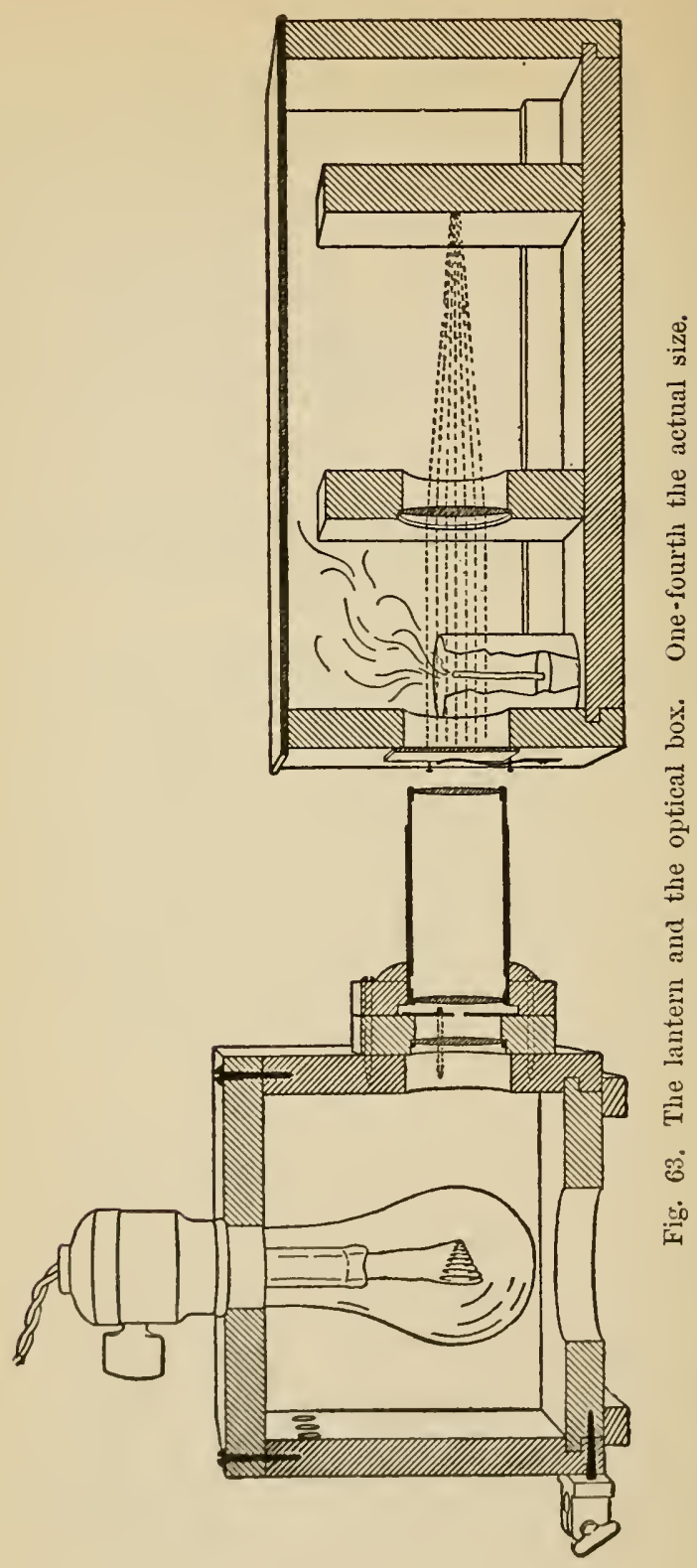


the hole in the cork. Put back the cork. Place the incense-holder in the optical box and put the glass lid on the box. Arrange the lantern to throw a beam of light through the window into the box. The smoke will be made luminous by the light so that the path of the rays can be seen. Make the rays parallel by pushing in the drawtube holding the outer projecting lens of the lantern. Set the plane mirror against the side of the box. Let the rays fall obliquely upon the mirror. Accurate measurement would show (1) that the incident ray, the reflected ray, and the perpendicular to the point of incidence, all lie in the same plane, and (2) that the angle between the incident ray and the perpendicular - angle of incidence - is equal to the angle between the perpendicular and the reflected ray - angle of reflection (Hero of Alexandria, about 100 B. C.).

\section{Reflection fron Concave Mirrors}

Principal Focus. - 1. Place the concave mirror (the polished inner surface of the segment of a sphere of $5 \mathrm{~cm}$. radius) at right angles to the pencil of parallel rays.

The rays will be reflected to a point $2 \frac{1}{2} \mathrm{~cm}$. from the mirror. ${ }^{1}$ This point, to which parallel

I Much smoke will make the rays less visible. The incident 
rays are converged, is the principal focus of the concave mirror. The distance between the principal focus and the reflecting surface is termed the principal focal distance; it is one half the radius of curvature. Accurate measurement would show that the angle between the incident ray and the perpendicular, ${ }^{1}$ in this case the radius of the spherical surface, equals the angle of reflection.

2. Take the mirror from the box and set it in the principal axis of the beam coming from the lantern. Replace the $2 \mathrm{~mm}$. diaphragm by the diaphragm with $L$-shaped aperture. At the principal focus of the concave mirror hold the small round screen with slender handle.

The inner rays of the beam will be intercepted by the screen. The outer rays will be reflected from the mirror and an inverted, real image of the L-shaped aperture will be seen upon the screen. The image will be smaller than the object. When the distance between the mirror and the object is less than the radius of curvature but greater than the focal distance, the image is real, inverted, and larger. With concave mirrors, real images are always inverted.

and the reflected bean may he compared by turning the mirror slightly, so that they lie side by sicle.

1 It is assumed that the spinerical surface is composed of an infinite number of plane surfices. 
3. Obtain a luminous point as follows. Insert in front of the condenser the diaphragm with $2 \mathrm{~mm}$. aperture. Place the glass slide over the window of the box. Pull out the draw-tube to make the pencil of rays convergent. Throw this convergent pencil into the box. Determine its focus by finding the place at which a clear image of the aperture of the diaphragm is formed upon a screen. This focus will serve as a luminous point.

After converging to the focus, the rays will diverge again. Place the mirror $2.5 \mathrm{~cm}$. from the luminous point. The luminous point will then lie at the principal focus of the mirror. Turn the mirror at a small angle with the axis of the pencil.

The reflected rays will be parallel.

Conjugate Foci. - 1. Place the mirror at a distance from the luminous point greater than the radius of curvature of the mirror.

The diverging incident rays will be reflected from the spherical surface to a point between the luminous point and the mirror. At this point a real image of the luminous point will be seen.

The point from which the rays diverge, and the point to which they converge by reflection from the mirror are termed conjugate foci.

2. Draw back the lantern and thus increase 
the distance between the luminous point and the mirror.

As the distance between the luminous point and the mirror increases, the distance between the mirror and the image diminishes.

Move the lantern towards the optical box, and thus bring the luminous point towards the mirror.

As the distance between the mirror and one conjugate focus diminishes, the distance between the mirror and the other conjugate focus increases. As one focus approaches the mirror, the other recedes.

3. Place the mirror $5 \mathrm{~cm}$. from the luminous point. The luminous point is now at the centre of the sphere of which the reflecting surface is a segment. The incident rays are therefore all radial, i. e. perpendicular, to this surface. Consequently all the rays will be reflected to the point of origin. The incident and the reflected rays will coincide. The centre of the reflecting surface and its optical image will also coincide. The conjugate foci coincide.

Virtual Image. - 1. Place the mirror at a distance from the luminous point less than the principal focal distance.

The reflected rays will diverge. They will appear to proceed from a point lying behind the mirror. The distance between this unreal or 
virtual image and the mirror will be greater than the distance between the mirror and the luminous point. As the luminous point approaches the mirror, its virtual image will also approach.

2. Hold a small object nearer the mirror than its principal focal distance.

Note that the image is virtual, upright, and larger than the object.

Construction of Image from Concave Mirrors. Determine by construction the length of the image of an arrow $2 \mathrm{~cm}$. long, placed $10 \mathrm{~cm}$. from the middle point of a concave mirror of $5 \mathrm{~cm}$. radius of curvature.

Draw a horizontal line. With any convenient point on this line as a centre describe an arc of $5 \mathrm{~cm}$. radius, that shall intersect the line. This arc will be the section of a concave mirror. The horizontal line will be the principal axis, and the intersection of the principal axis and the arc, the middle point of the mirror. The principal focus of the mirror will lie halfway between the centre of curvature and the middle point. At right angles to the principal axis and $10 \mathrm{~cm}$. from the middle point draw a vertical arrow $2 \mathrm{~cm}$. long. Determine first the position of the image of the point of the arrow. Draw from the point to the mirror an incident ray parallel to the principal axis. This parallel ray 
will be reflected through the principal focus. Draw a second incident ray from the arrow point through the centre of curvature. This ray will be perpendicular to the spherical surface and will be reflected in the same line. The intersection of these two reflected rays will be the image of the point of the arrow.

Determine in like mamner the position of the image of the other end of the arrow.

\section{Reflection from Convex Mirrors}

The laws of reflection from convex mirrors may be deduced from those already stated for concave mirrors. The image reflected from convex mirrors is virtual, upright, and smaller than the object.

Determine by construction the length of the image of an arrow, $2 \mathrm{~cm}$. long, placed $10 \mathrm{~cm}$. from the middle point of a convex mirror of $5 \mathrm{~cm}$. radius.

\section{Pefraction}

1. Place the diaphragm of $2 \mathrm{~mm}$. aperture in front of the condenser. Push in the draw-tube of the lantern until a heam of parallel rays enter the box. In the box lay the square glass bottle on its side upon a wooden block and at right 
angles to the pencil of light. Neglect the reflected rays.

Observe that the incident rays pass through the bottle and its contents, and are not bent from their course. Light, passing from one medium into another of different density, is not refracted, provided the course of the ray be perpendicular to the surface separating the media.

2. Turn the bottle so that the incident ray shall enter it at an angle.

On passing from the air into the denser medium of the glass and the contained liquid, the incident ray will be bent from its course. On passing from the denser medium into the air again, the ray will once more be bent from its path. Imagine a perpendicular erected at the points of incidence and emergence. The refracted ray will be bent toward the perpendicular on passing into the denser medium, and away from the perpendicular on leaving the denser medium.

Turn the bottle and thus alter the angle between the incident ray and the perpendicular (angle of incidence).

The angle between the refracted ray and the perpendicular (angle of refraction) increases with the angle of incidence. Exact measurements made by Snellius and Descartes, about 1621, 
showed that - (1) the refracted ray lies in the same plane with the incident ray and the perpendicular, and (2) the sine of the angle of incidence stands in an unalterable relation to the sine of the angle of refraction.

The sine of the angle of incidence is to the sine of the angle of refraction as the velocity of the light ray in the first medium is to its velocity in the second, or refracting medium. The ratio of the velocity of light in a vacuum to its velocity in any medium is termed the index of refraction, or refractive power of that medium. If the velocity of light in a vacuum be taken as 1 , that of light in air at $0^{\circ}$ temperature and $760 \mathrm{~mm}$. pressure, will be 0.9997 , a difference so slight that the velocity in air is usually taken as the unit. The law of refraction is commonly expressed as follows: Let $n$ represent the index of refraction, $a$ the angle of incidence, and $b$ the angle of refraction; then

$$
\sin a=n \sin b, \text { or } n=\frac{\sin a}{\sin b} \text {. }
$$

As a rule, the physically denser medium is also optically denser. Thus the refractive index for the Frauenhofer line ${ }^{1} \mathrm{D}$, on passing from air

1 White light is composed of rays of different refrangibility; hence the use in such measurements of pure spectral rays. 
into crown glass, of which spectacle lenses are made, is 1.530 ; into flint glass, 1.635 ; into water at $15^{\circ} \mathrm{C} ., 1.332$.

\section{Refraction by Prisms}

A refracting medium bounded by two plane surfaces not parallel is termed a prism. The planes are termed the refracting surfaces. The angle which they make with each other is termed the refracting angle of the prism.

1. Place a prism in the optical box in the beam of parallel rays. The beam will be bent from its course on entering and on leaving the prism. The emerging pencil will be divergent, for the homogeneous rays, the union of which produces the sensation of white light, are not equally refracted, - the rays towards the red end of the spectrum are bent less strongly than those towards the violet end, the order being red, orange, yellow, green, blue, violet.

Construction of the Path of a Ray passing through a Prism. - Draw a horizontal line $5 \mathrm{~cm}$. in length. Upon this line construct the section of a prism. ${ }^{1}$

1 To construct the scction of a prism: Let the horizon. tal line be the base of the prism. Place the brass leg of the drawing compasses at one end of the base line. Draw a circle of $3 \mathrm{~cm}$. radius. Place the brass leg at the other end of the base line and draw a circle of the same radius. A line joining the intersections of the two circles will be perpendicular to the 
Draiv with ink a ray incident to the refracting surface. ${ }^{1}$ Find the sine of the angle of incidence. $^{2}$ For the Frauenhofer line $\mathrm{D}$ passing from air into crown glass the ratio of the sine of the angle of incidence an to the sine of the angle of refraction $b n$ is

$$
\text { an }: \text { bn }:: 1.53: 1
$$

For the same light passing from crown glass into air the ratio of the sine of the angle of incidence to the sine of the angle of refraction is the reciprocal of the ratio from air to crown glass

$$
\text { an }: \text { bn }:: 1: 1.53
$$

middle of the base line. Let a point on this perpendicular $5 \mathrm{~cm}$. above the hase line he the apex of the section. Join the apex with the ends of the base line. Ink the boundary lines of the cross-section thus obtained. Erase the pencil construction lines.

1 For convenience let the incident ray come from the prolonged base line of the prism $10 \mathrm{~cm}$. from the nearest refracting surface. Let the point of incidence - the point at which the incident ray meets the refracting surface - be about the midtle of the refracting surface.

2 To find the sine: With the point of incidence as a centre draw a circle of convenient radins (2 emr.). Construct a radius of this circle perpendicular to the refracting surface at the point of incidence. From the intersection of the circle with the ineident ray draw a line perpendieular to the radius (a line drawn from the roint of intersection parallel to the refracting surface will be perpendicular to the radius). This is the sinus line of the angle. The ratio of this line to the radius of the circle is the sine of the incident angle. 
INTRONUCTION TO PHYSIOLOGICAL OPTICS 415

Measure the length of the sine of the angle of incidence in millimetres. Suppose that an in the present instance is $13 \mathrm{~mm}$. Then with - equation (1)

$$
1.53: 1:: 13: x
$$

$x=8.5 \mathrm{~mm}$., the sine of the angle of refraction.

Find on the construction circle within the prism a point $8.5 \mathrm{~mm}$. in a perpendicular line above the diameter at right angles to the refracting surface. Continue the ray through this point to the second refracting surface. Ink the path of the ray within the prism. Erase the construction lines within the prism, but leave untouched those without the prism. Find the sines of the angles of incidence and refraction at the second refracting surface. Draw with ink the path of the emergent ray. Preserve all these construction lines. Write the equations in ink in the upper left-hand corner of the paper, and the four sines in the upper right-hand corner.

The degree to which light is refracted on passing through a prism depends on the refracting power of the substance of the prism, the size of the refracting angle, and the size of the angle of incidence. 


\section{Refraction by Convex Lenses}

Principal Focus. - 1. Place the diaphragm of 2 min. aperture in the lantern. Throw a beam of parallel rays into the optical box. Place the double convex lens in the axis of the beam about $5 \mathrm{~cm}$. from the window of the box.

The parallel rays will be brought to their principal focus about $10 \mathrm{~cm}$. (4 inches) from the lens. Note the increase in intensity as the rays converge.

Place the black wooden screen at this point.

A real image of the luminous aperture of the diaphragm will be perceived.

2. Place the diaphragm of $2 \mathrm{~mm}$. aperture over the window of the box. Direct the light of the lantern upon the opening in the diaphragm. From the illuminated opening rays will diverge in all directions. Place the lens $10 \mathrm{~cm}$. from this luminous body, so that it shall lie in the principal focus of the lens.

The diverging rays will be rendered parallel. Rays diverging from the principal focus are rendered parallel by passing through a convex lens.

A lens may be regarded as an infinite series of prisins. In a convex lens the refracting angle 
of each hypothetical prism is directed to the periphery of the lens. As the periphery is approached the refracting angles increase, and hence refraction increases. The increased refraction of the outer rays diverging from a luminous point compensates in part for their greater angle of incidence, and hence most of the rays converge approximately to the same focus.

Estimation of Principal Focal Distance. - Remove from the lantern the tubes holding the projection lenses. Place in front of the condensing lens the diaphragm with L-shaped aperture each limb of which is $5 \mathrm{~mm}$. long and $1 \mathrm{~mm}$. broad. Place the convex lens in the axis of the pencil emerging from the illuminated slit and at a distance from it a little greater than the principal focal distance as determined roughly in the preceding experiment. On the other side of the lens place a screen at such a distance as to give a strongly enlarged clear picture of the $L$. Measure

$l=$ the length of one limb of the $L$,

$L=$ the length of its image,

$A=$ the distance of the screen from the lens,

$f=$ the principal focal distance of the lens,

then ${ }^{1}$

$$
f=A \frac{l}{L+l}
$$

1 This formula is derived as follows; Let $a$ be the distance. 
The principal focal distance of a double convex Iens is approximately equal to the radius of curvature.

Conjugate Foci. - Place in the lantern the diaphragm of $2 \mathrm{~mm}$. aperture. Remove the tubes holding the projecting lenses. Place the convex lens against the window of the optical box. Place the black screen twice the focal distance from the lens. Move back the lantern until a clear image of the luminous aperture appears on the screen.

The point from which rays passing through a lens diverge, and the point to which they converge, are termed conjugate foci. Measure the distance of the luminous aperture from the lens. It will be found to be twice the focal distance. When the point of divergence is separated from the lens by twice the focal distance, the point of convergence is equally distant from the other

of the object from the principal surface of the lens (see prage 46); then $\frac{1}{A}+\frac{1}{a}=\frac{1}{f}$. The relation between the size of the image and the size of the object is $L: l:: A: a$; then $\frac{1}{a}=\frac{L}{A l}$, and, by substitution, $\frac{1}{f}=\frac{1}{A}+\frac{L}{A l}$, whence $f=A \frac{l}{L+l}$. Compared with the thickness of the lens, the distance of the object from the lens is so great that it may he used in place of the unknown distance from the principal surface (Kohlrausch: Leitfarlen der praktischen Physik, 1887, p. 142). 
side of the lens, - the conjugate focal clistances are equal.

Move the lantern farther from the lens.

The conjugate focus will approach the lens. As one conjugate focus recedes the other approaches the lens.

Virtual Image. - 1. Place the $2 \mathrm{~mm}$. diaphragm over the window of the optical box. Let the diverging rays pass through the convex lens placed at a distance from the luminous point less than the principal focal distance.

After passing through the lens, the diverging rays will continue to diverge though the degree of divergence will be less. Prolonged backwards, they would unite in a virtual image on the same side of the lens as the luminous object. The virtual image is farther from the lens than the object, is never inverted, and is always enlarged. (Compare the construction, directions for which will be given on page 421 .)

2. Look through the convex lens at printed words placed between the lens and its principal focus.

The image is virtual and enlarged.

Construction of Image obtained with Convex Lens. - The line which joins the centres of curvature of a double convex lens is termed the principal axis or optical axis. In every lens there 
are in the principal axis two points so placed that when the entering ray is directed toward the first, the emergent ray will appear to come from the second in a direction parallel to the entering ray. These are termed nodal points. In ordinary glass lenses the distance between the two nodal points is about one third the thickness of the lens. When this distance is so small that it may be disregarded, the two nodal points may be assumed to meet in an intermediate point termed the optical centre (compare page 444) of the lens. A ray directed to the optical centre is not refracted but passes through the lens in a straight line. The position and size of an image formed by a lens can be found by drawing one line from each extremity of the object through the optical centre, and another from each extremity parallel with the principal axis to the lens and thence through the principal focus. The intersections of these lines mark the position of the image and its upper and lower limit. It is necessary to remember that parallel rays are refracted through the principal focus only when the aperture of the lens does not exceed approximately ten degrees.

Draw a horizontal line to serve as the principal axis. Let a point near the middle of the line be the optical centre of a double convex lens of $10^{\circ}$ aperture and $5 \mathrm{~cm}$. radius. The radius of curva- 
ture being $5 \mathrm{~cm}$., the principal focus will lie approximately $5 \mathrm{~cm}$. from the optical centre. Draw through the optical centre a line $10 \mathrm{~mm}$. long at right angles to and bisected by the primcipal axis. Connect the ends of this line with the principal focus. The angle included will be approximately $10^{\circ}$. The vertical line will then represent a double convex lens of $10^{\circ}$ aperture, assumed to be without thickness in order that the nodal points may coincide with the optical centre. At any distance greater than $5 \mathrm{~cm}$., draw an arrow at right angles to and bisected by the principal axis. The height of the arrow must not exceed the diameter of the lens, so that rays emitted from the ends of the arrow parallel to the principal axis of the lens shall pass through the lens. From the ends of the arrow draw to the lens and thence through the principal focus incident rays parallel to the principal axis. From each end of the arrow draw a line through the optical centre of the lens.

The intersections of these lines mark the upper and lower limits of the image. Note that the image is real and inverted. If the object be situated at twice the focal distance from the lens, the image will be the size of the object; if at less than twice the focal distance, the image will be 
larger than the object; if at more than twice the focal distance, the image will be smaller than the object; finally, if the object be situated between the principal focus and the lens, the image will no longer be real, but virtual and larger than the object, as mentioned on page 419 .

\section{Refraction by Concave Lenses}

Place the diaphragm with $2 \mathrm{~mm}$. aperture in front of the condenser. Throw a pencil of parallel rays into the box. Let the rays fall upon a concave lens.

The parallel rays will be rendered divergent.

Look through the concave lens at printed words. The image is virtual, upright, and smaller than the object. It is nearer the lens than the object, and is always within the principal focal distance.

\section{Refraction by Segments of Cylinders}

1. Place the diaphragm with $2 \mathrm{~mm}$. aperture in front of the condenser. Throw a pencil of parallel rays into the box. Place the cylindrical lens in the axis of the pencil in such a position that the curvature shall be from side to side, $i$. $e$. in the horizontal meridian. 
INTRODUCTION TO PHYSIOLOGICAL OPTICS 423

The image of the circular aperture in the diaphragm will be a vertical line with blurred convex ends.

Turn the cylinder so that the curvature shall be in the vertical meridian.

The image will be a horizontal line with blurred convex ends.

2. Place the diaphragm with horizontal slit in the lantern. Throw parallel rays into the box. Place the cylinder in the axis of the pencil with its curvature vertical.

The horizontal line is a fusion of illuminated points. From each point rays diverge in all directions. Those passing from any point in vertical planes throigh the cylinder convex in its vertical meridians will be focussed by the convex surface in a corresponding point in the image. The overlapping of such points will form a horizontal line with clear upper and lower edge. The rays passing from any point in the illuminated line in horizontal planes through the cylinder with vertical curvature will be refracted by plane glass surfaces and will not come to a point but will form a faint horizontal line. The overlapping in the image of the bright points in which unite the rays passing in vertical planes and the faint horizontal lines formed by rays passing in horizontal planes will 
form upon the screen a horizontal line with blurred ends.

Place the vertical slit in front of the condenser.

A broad, faint, horizontal line with blurred ends will be observed. Draw a diagram illustrating the formation of this image.

Turn the cylinder, so that the curvature shall lie in the horizontal meridian.

The horizontal rays are at once united in a narrow sharply defined vertical line with blurred ends.

\section{Refraction through Combined Convex and}

\section{Cylindrical Lenses}

Thus far segments of perfect spheres or cylinders have been considered separately. In the eye both the cornea and the lens are frequently more convex in one meridian than in another. Such surfaces can be obtained by combining a convex with a cylindrical lens.

1. Place the diaphragm of $2 \mathrm{~mm}$. aperture in front of the condenser. Throw parallel rays into the box. Place the convex lens in the axis of the pencil next the window. Receive the image of the illuminated aperture upon a screen placed at the principal focus. The image will be a well-defined circle. Place the cylindrical lens 
INTRODUCTION TO PHYSIOLOGICAL OPTICS 42 כ̃

as close as possible to the convex lens. Let the curvature of the cylinder be in the vertical meridian.

The circle will give place to a vertical line.

Move the screen about $4 \mathrm{~cm}$. nearer the lenses.

The image of the circle will now be a horizontal line.

Place the screen half way between the nearer and the farther focal lines.

The image will be circular. At other points in the focal interval or space separating the two focal lines the image will be an ellipse.

2. Hang the block containing the cylindrical lens on the end of the draw-tube of the lantern. Leave the convex lens in its former position. Fill the box with smoke. Let the currature of the cylindrical lens be in the vertical meridian. Observe the pencil of rays.

The pencil will be drawn out to a vertical line at the farther focus. Seen from above the crosssection of this line will be a bright spot. At the nearer focus the pencil will be flattened to a horizontal line.

Rotate the cylinder through $90^{\circ}$. The curvature will now be in the horizontal meridian. Watch the pencil as the lens turns.

As the cylinder revolves the contour of the pencil will change. When the curvature is 
finally horizontal the nearer focal line will be vertical, the farther focal line will be horizontal.

Rotate the cylinder through $45^{\circ}$.

By looking at the pencil first from one side of the box and then from the other, the focal lines may readily be seen in profile, as well as in cross-section.

\section{Aberration}

Spherical Aberration by Reflection. - In Fig. 64 a concave mirror, $A B$, has the centre of curvature, $C$, and the principal focus, $F . \quad D E$ is one of several incident parallel rays. $C E$ is perpen. dicular to the point of incidence. $E F$ is the ray reflected from $E$ to the principal focus, $G$ the

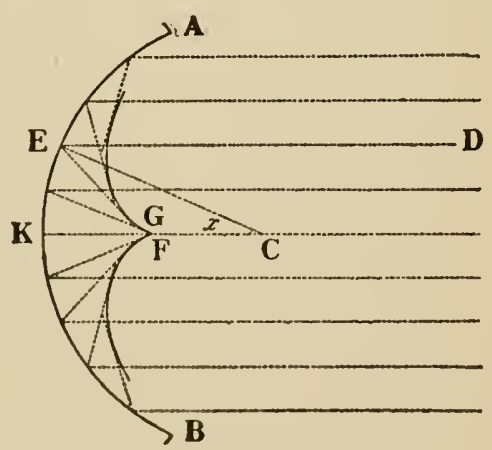

Fig. 64 .

point at which the reflected ray cuts the axial line $C K . \quad D E \| C G$, therefore $\angle G C E=C E D$ 
$=C E G$, and $C G E$ is an isosceles triangle. Then if $r$ be the radius and $x$ the angle formed by the perpendicular $C E$ with the axial ray $C G, C G$ $=\frac{r}{2 \cos x}$. So long as angle $x$ is small, cosine $x$ will be nearly 1 . $C G$ will then be nearly one half the radius $C K$. Hence incident rays near the axial ray $C K$ will be reflected approximately to the principal focus $F$, which lies half way between the centre of curvature and the mirror. As the aperture ${ }^{1}$ of the mirror increases, angle $x$ also increases. The larger $x$, the smaller will be the denominator of the expression for $C G$, and the greater the distance of $G$ from $C$. Rays reflected from the outer portion of a mirror of larger aperture meet the principal axis nearer the mirror than those refiected from the central portion. The intersection of the reflected rays produces a curved line - the caustic curve or focal line. By revolving Fig. 2 about the axis $C K$, a caustic or focal surface will be obtained. ${ }^{2}$

Spherical Aberration by Refraction. - 1. The observations just made concerning concave mirrors are applicable also to lenses. Rays entering

1 The aperture is the angle included between lines drawn from the principal focus to the margins of the mirror or lens.

2 Jochmann and Hermes. Grundriss der Experimentalphysik, 1890, p. 153. 
a lens with aperture greater than $10^{\circ}$ are not refracted to the principal focus but cross the principal axis between the principal focus and the lens. The caustic surface formed by the intersection of these peripheral rays may readily be shown with any lens or cylinder of small radius of curvature.

2. Place the diaphragm with $2 \mathrm{~mm}$. aperture in front of the condenser. Throw parallel rays into the optical box. Set in the box near the window the cylindrical bottle of clear glass filled with water. The bottle will serve as a powerful refracting cylinder.

The circular pencil of parallel rays will be brought to a focus in a vertical line (compare page 422). The outer rays of the pencil pass through the outer portion of the cylinder, and are therefore more strongly refracted than those near the optical axis. Each refracted ray intersects the refracted rays nearer than itself to the principal axis. These intersections form two curved surfaces extending from the principal focus - in this case a vertical line - towards the cylinder. On regarding these surfaces from above, their curvature will be apparent.

3. Remove the projecting lenses; place the ground glass plate and the diaphragm with $2 \mathrm{~mm}$. aperture in frout of the condenser. Let the rays 
diverging from the illuminated aperture pass through the refracting cylinder.

The curvature of the caustic surfaces will be more noticeable than in Experiment 2.

Dispersion Circles. - 1. Let the parallel rays pass through the double convex lens. Place a screen at the principal focus. A clear image of the circular aperture in the diaphragm will be seen. Move the screen away from and then towards the lens.

When the screen is either nearer or farther from the lens than the principal focus, the image will be larger and less distinct. The screen will cut the pencil in the one case before it has converged to the focus, and in the other case after it has passed the focal point and is diverging. Under such circumstances the image of a point becomes a circle, termed a dispersion circle or circle of confusion.

2. Substitute the diaphragm with L-shaped aperture for that with circular aperture. Place the screen a little nearer or farther than the focal point.

The image will be a broad blurred line with convex ends. The pencils proceeding from each luminous point in the line will fall upon the screen in dispersion circles. The broad line is caused by the overlapping of the dispersion cir- 
cles. Similar blurring by dispersion circles is caused by the rays which pass through the outer parts of a lens coming to a focus sooner than the axial rays.

Myopia. - In the normal eye at rest parallel rays are brought to a focus upon the retina. In the myopic eye parallel rays, and even rays to a certain degree divergent, are brought to a focus in the vitreous, whence they fall in dispersion circles on the retina. The most common cause of myopia is the abnormal length of the anteroposterior diameter of the eye. The defect can be remedied by placing a concave lens before the eye. The entering rays are thereby rendered divergent, or their divergence is increased, so that their focus is displaced backwards towards the retina. The degree of the myopia is measured by the strength of the concave lens which, placed before the eye, will bring the principal focus exactly to the retina.

Let parallel rays pass through the convex lens of $10 \mathrm{~cm}$. (4 inch) focal distance placed against the window of the optical box. Find the principal focus and then move the screen $2.5 \mathrm{~cm}$. farther from the lens.

The image will be blurred. The screen will intersect the rays diverging from the focal point. Hold the weak concave lens, marked -2 , in 
INTRODUCTION TO PHYSIOLOGICAL OPTICS 4.31

front of the window. This lens has a focal distance of two dioptres or one-half metre (see page 435 ).

The image will be clear again. The myopia in this case is therefore $-2 \mathrm{D}$.

Hypermetropia. - In the hypermetropic eye at rest parallel rays and even those to a certain degree convergent meet the retina before they have come to a focus. The most frequent cause of hypermetropia is the abnormal shortness of the antero-posterior diameter of the eye. The defect can be remedied by placing a convex lens before the aye. The entering rays are thereby rendered convergent, or their convergence is increased. The degree of the hypermetropia is measured by the strength of the convex lens which, placed before the eye, will so increase its convergent power that parallel rays will come to a focus on the retina.

Place the screen $2.5 \mathrm{~cm}$. nearer the lens than the principal focus.

The image will be blurred. The screen will intersect the rays before they have converged to the focal point.

Hold the weak convex lens, marked +2 , in front of the window.

The image will be clear. The hypermetropia in this case is therefore $+2 \mathrm{D}$. 
Myopia and hypermetropia will be further considered under refraction in the eye.

Chromatic Aberration. - The velocity of the homogeneous spectral rays composing white light is believed to be the same in a vacuum and in gases, but differs in transparent liquids and solids. The front of the light wave strikes the refracting surface obliquely. As the wave front enters the medium, its speed lessens. Thus the part of the front which enters first travels in the refracting medium at a speed less than the remainder which has not yet entered. The wave front is therefore bent towards the retarled portion. The shorter the wave length, $i . c$. the greater the wave number, the slower will the wave advance in the refracting medium. Hence the wave front of the violet ray moves more slowly in the medium than the front of the red ray, and is therefore bent more from its course. The reverse of this process takes place when the wave emerges from the refracting medium. The violet rays are therefore more refrangible than the red, and on entering a refracting medium pursue a different path. Thus each spectral ray passing through a lens has its own principal focus. In other words, the images for the several spectral colors do not coincide precisely. The order in which the refracted spectral rays cross the principal axis is that of 
their refrangibility; violet crosses nearest the lens, then blue, green, yellow, orange, and red in the order named. The principal focus will thus be a line of colors lying in the principal axis, the end nearest the lens being violet. The peripheral portions of a lens refract rays parallel to the principal axis more strongly than the axial portion. Hence the chromatic aberration will increase with the aperture of the lens.

Put the ground glass plate and the diaphragm with $2 \mathrm{~mm}$. aperture in front of the condenser. Let the rays from the illuminated spot of ground glass pass through the $10 \mathrm{D}$ lens placed about $15 \mathrm{~cm}$. in front of the ground glass, i.e. a distance somewhat greater than the focal distance of the lens $(10 \mathrm{~cm}$.). Place a white screen about $15 \mathrm{~cm}$. in front of the lens.

The image of the white spot upon the ground glass will be a disk with violet centre and red margin.

Remove the white screen farther from the lens. At a distance of about $30 \mathrm{~cm}$. the centre of the image will be red and the border violet.

The image in this experiment is blurred because the rays which pass through the peripheral portion of the lens cross the principal axis sooner than the rays which pass through the axial portion. If the screen be placed at the focus of the 
more axial rays, this focal point in the image will be surrounded by dispersion circles made by the rays which have been refracted from the periphery through foci nearer the lens and which are now diverging from these foci. If the screen be placed at the principal focus for the peripheral rays, this focal point will be surrounded by dispersion circles male by the rays that have not yet converged to the principal axis. (Compare spherical aberration, page 428.)

Aberration avoided by a Diaphragm. - Place before the condenser the paper diaphragm with $1 \mathrm{~cm}$. aperture.

The image at once becomes distinct, and the colors practically disappear. The outer rays, which when refracted would cross the principal axis far enough from the principal focus to cause dispersion circles, have been cut off. When the aperture of a lens or mirror is reduced by a diaphragm to $10^{\circ}$, the greater part of the spherical aberration is prevented.

Spherical aberration is still further reduced by combining several lenses in an objective (aplanatic system).

With an achromatic lens, consisting of a collecting lens of crown glass united with a dispersing lens of flint glass, all the spectral rays may be brought to the same focus, and chromatic aberration altogether avoided. 


\section{Numbering of Prismis and Lenses}

Numbering of Prisms. - Prisms may be numbered according to refracting angles or according to the extent to which they turn the light ray from its course (angular deviation). Angular deviation is expressed by the methods of Dennett and of Prentice.

Dennett's method. - The length of an are of $57.295^{\circ}$ equals its radius of curvature. A prism which will bend the ray one hundredth part of this arc is called one centrad. The angular deviation produced by the prisms are by this methor expressed in hundredths of the radius measured on the arc.

Prentice's method. - The unit of comparison is a prism-dioptre, i.e. a prism that deflects a ray of light one centimetre at a plane one metre distant, or, in other words, the hundredth part of the radius measured on the tangent.

Numbering of Lenses. - Lenses are numbered according to their refractive power. The unit is a leus with a focal distance of one metre. This unit is termed a dioptre, D. A lens of two metres focus is one half the refractive power, or $\frac{1}{2} \mathrm{D}$. The lenses ordinarily employed in ophthalmic practice extend from $0.12 \mathrm{D}$ to $22 \mathrm{D}$. The 
principal focal distance of any lens in the dioptric system may be found by dividing one metre, or $100 \mathrm{~cm}$., by the number of dioptres; thus the focal distance of a lens of $4 \mathrm{D}=\frac{10}{4} \underline{0}=25 \mathrm{~cm}$.

Convex lenses are marked + , concave lenses -. If two or more lenses are placed together, the dioptric power of the system thus formed equals the algebraical sum of the dioptric powers of the lenses in the system. 


\section{IX \\ REFRACTION IN THE EYE}

The Eye as a Camera Obscura. - 1 . From the eye of an ox remove the posterior part of the sclerotic and choroid coats over an area about $1 \mathrm{~cm}$. in diameter near the outer (temporal) side of the optic nerve. Cover the retina with a watch glass. Turn the cornea towards an incandescent lamp.

A small, real, inverted image of the lamp will be seen upon the transparent retina. In the white rabbit the choroid has so little pigment that the retinal image may be seen without removing the outer coats of the eye.

2. In a darkened room, direct a blond, blueeyed individual to turn the eyes so that one cornea shall lie in the outer angle of the eye. Hold a candle near the temporal side of that eye.

The small, inverted retinal image of the candle can often be seen shining through the sclerotic coat at the nasal side of the eye. 


\section{The Schematic Eye}

In passing from the external air to the retina, the rays of light undergo refraction at the layer of tears on the anterior surface of the cornea, the surfaces bounding layers of unequal refractive power in the substance of the cornea, the anterior surface of the aqueous humor, the anterior surface of the lens, the surfaces bounding layers of unequal refractive power in the substance of the lens, and the anterior surface of the vitreous humor. To determine the size and position of visual images, it is fortunately not necessary to calculate refraction at each of these many surfaces. The problem is much simplitied by the following considerations.

The irregularities in the refractive power of the different parts of the cornea are small; and the refractive index of the layer of tears which covers the anterior surface is almost identical with the index of the substance of the cornea and that of the aqueous humor. Practically therefore the layer of tears, the cornea, and the aqueous humor may be regarded as a single refracting medium. Further, although the layers of which the lens is composed increase in refracting power towards the centre of the lens, it is 
known that the error introduced by assuming the lens to be homogeneous is unimportant. Thus the simplified dioptric system of the eye consists of three refracting surfaces: the anterior surface of the cornea, ${ }^{1}$ the anterior surface of the lens, and the anterior surface of the vitreous humor. The index of refraction of the aqueous and vitreous humors is practically the same.

The several refracting surfaces of this optical system are approximately "centred," $i$. e. placed with their centres of curvature upon a right line, the optical axis. The diaphragm (iris) is of such a size and position that the rays entering the eye intersect the axis at small angles; the aperture of the system is therefore small. Under such conditions it is possible to find upon the principal axis of the system certain cardinal points, discovered by Gauss, by the aid of which the situation and size of the visual images may be determined. The cardinal points are (1) the anterior principal focus, (2) the anterior principal point, (3) the posterior principal point, (4) the anterior nodal point, (5) the posterior nodal point, (6) the posterior principal focus. These points are reciprocal.

As the dioptric system of the eye consists of a

1 For convenience, the anterior surface of the cornea will be held to include the layer of tears. 
spherical surface ${ }^{1}$ (the cornea) and a double convex lens (the crystalline lens) it will be advisable to consider first the cardinal points of the cornea (System A), next those of the lens (System B), and finally those of the two combined as in the eye (System C).

\section{Cardinal Points of the Cornea (System A)}

Construction Drawing of System A. - Draw a horizontal line to serve as the optical axis. ${ }^{2}$ Take any point, $k$, in this line for a centre of curvature. $^{3}$ From this point describe an arc

1 The cornea is not strictly a spherical surface, but more nearly that produced by the revolution of an ellipse about its major axis.

2 This construction drawing should be placerl near the top of the page, in order to permit the construction drawings for the lens and the compound optical system to be marle beneath it. All these drawings will be the natural size.

3 The following list will be found convenient:

$k$, centre of curvature.

$r$, rarlius of curvature.

$h_{1}$, intersection of the first spherical surface of any system with its principal axis ("first" is used in the sense of nearest the source of light).

$h_{2}$, intersection of the second spherical surface with its principal axis.

$n_{1}$, the first medium, that which bounds the refracting surface on the sille from which the ray comes; also the refractive index of this medium.

$n_{2}$, The second medium, that which bounds the refracting surface on the side from which the ray emerges; also the refractive index of this medium. 
with the radius $r=7.829 \mathrm{~mm}$., the radius of curvature of the cornea. The intersection of the arc with the axis is termed the principal point $h_{1}$ of the axial ray. The spherical surface separates two media: $n_{1}$, the air, and $n_{2}$, the aqueous humor.

Principal Focal Distances. - 1. Rays passing from the first medium through the cornea into the second medium unite nearly in a point, the posterior principal focus, $\phi_{2}$. The distance, $h_{1} \phi_{2}$, between this point and the principal point is the posterior principal focal distance, $F_{2}$. Rays passing from the aqueous humor through the cornea

$\phi_{1}$, anterior principal focus.

$\phi_{2}$, posterior principal focus.

$F_{1}$, anterior focal distance.

$F_{2}$, posterior focal distance.

$f_{1}$, anterior conjugate focal distance.

$f_{2}$, posterior conjugate focal distance.

$o$, optical centre.

$K_{1}$, first nodal point.

$K_{2}$, second nodal point.

$H_{1}$, first principal point.

$\mathrm{H}_{2}$, second principal point.

$s$, point between two lefracting surfaces at which an object must be in order that the inages of the ohject formed by the refracting surfaces shall be sinilar, $i . e_{\text {. }}$, inuages of one another, lying therefore in the principal surfaces.

Where confusion might arise in applying these terms to System $\mathrm{A}, \mathrm{B}$, or $\mathrm{C}$, they will be distinguisher by placing with them the letters $A, B, C$, respectively. Thus the anterior focal distance of the lens will be written $F_{1} B$, wherever it might otherwise be confused with that of System $\mathrm{A}$ or $\mathrm{C}$. 
to the air, parallel to and near the axis, unite in front of the cornea nearly in a point, the anterior principal focus, $\phi_{1}$. The distance, $h_{1} \phi_{1}$, between this point and the principal point is the anterior principal focal distance, $F_{1}$. The principal focal distances are proportional to the coefficients of refraction of the first and last media. The posterior principal focal distance is calculated by the formula ${ }^{1}$

$$
F_{2}=\frac{n_{2} r}{n_{2}-n_{1}}
$$

In comparing refractive powers the air, $n_{1}$, is taken as the unit. Thus the formula becomes

$$
F_{2}=\frac{n_{2} r}{n_{2}-1}
$$

The anterior principal focal distance is calculated by tne formula

$$
F_{1}=\frac{n_{1} r}{n_{2}-n_{1}}
$$

As $n_{1}=$ unity, the formula becomes

$$
F_{1}=\frac{r}{n_{2}-1}
$$

The refractive index, $n_{1}$, of the air $=1$; accord-

1 For the derivation of the formulas in this chapter the reader is referred to the works of Donders (Accommodation and Refraction of the Fye) and Helmholtz (Handbuch der pliysiologischen Optik). 
ing to Helmholtz, ${ }^{1}$ the refractive index, $n_{2}$, of the aqueous humor is 1.3365 ; the ratio is $\frac{3}{4}$.

Calculate $F_{1}$ and $F_{2}$. The result is, $F_{1}=23.266$, $F_{2}=31.095$.

2. The principal foci may also be approximately found by construction. Erect at the principal point and the nodal point ${ }^{2}$ perpendiculars to the optical axis. Set off on each perpendicular distances from the optical axis proportional to the rapidity of light in the first and second medium. The ratio in the case of the air and the aqueous humor is $4: 3$. Mark therefore points $20 \mathrm{~mm}$. and $15 \mathrm{~mm}$. from the axis. Draw a line from the $20 \mathrm{~mm}$. point of the first perpendicular through the $15 \mathrm{~mm}$. point of the second, and produce the line to the optical axis. Its intersection with the optical axis is the posterior principal focus. Find in a similar way the anterior principal focus. Indicate upon the axis the cardinal points, remembering that the construction drawing is to be the natural size.

Construction of Image. - About $10 \mathrm{~cm}$. in front of the cornea draw an arrow, $i j$, which shall intersect the optical axis at right angles.

1 The figures for this and subsequent calculations under "Pefraction of the Eye," are those given by Helmholtz. They are collected in a convenient Table on pages 461 and 462 .

2 The centre of curvature is the nodal point of a system consisting of a single spherical surface. 
Draw a line from the point $i$ of the arrow through $k$. This line, since it passes through the centre of curvature, will coincide with the perpendicular to the refracting surface, and therefore will not be refracted. Draw from the point $i$ to the cornea a line parallel to the axis. This parallel ray will be refracted through the posterior principal focus $\phi_{2}$. The two rays will unite at their point of intersection, $i_{2}$, which point is the image of $i$ and is its conjugate focus. The arrow $i j$ was vertical to the axis. Hence its image will also be vertical to the axis. Draw, therefore, from $i_{2}$ a line vertical to the axis. From the end $j$ of the arrow draw a line throngh 7. The intersection of this line with the vertical line just drawn will be the image $j_{2}$ of the point $j$.

Calculation of the Position of the Conjugate Foci. - The conjugate foci may be found by the following formulas. Let $f_{1}$ be the conjugate focal distance $h_{1} i$, and $f_{2}$ be the conjugate focal distance $h_{1} j_{2}$.
(3 a) $f_{1}=\frac{f_{2} F_{1}}{f_{2}-F_{2}^{\prime}}$
(4 a) $f_{2}=\frac{f_{1} F_{2}}{f_{1}-F_{1}}$

For virtual images the formulas become
(3 b) $f_{1}=\frac{F_{1} f_{2}}{F_{2}^{\prime}-f_{2}}$
(4 b) $\quad f_{2}=\frac{F_{2} f_{1}}{F_{1}^{\prime}-f_{1}}$ 


\section{Cardinal Points of the Cristalline Lens} (Systen B)

Construction Drawing of System B. - When the lens is accommodated for distant vision the radius of the anterior surface is about $10 \mathrm{~mm}$., the radius of the posterior surface about $6^{\circ} \mathrm{mm}$; the thickness at the principal axis $3.6 \mathrm{~mm}$. The index of refraction of the lens is 1.4371. The index of the aqueous and vitreous humors is 1.3365 .

Beneath the construction drawing of the cardinal points of the cornea (System A) draw a horizontal line parallel to the optical axis of the cornea. This line will serve as the optical axis of the lens. From the intersection of the cornea with its optical axis let fall a perpendicular to the optical axis of the lens.

The anterior surface of the lens intersects the optical axis $3.6 \mathrm{~mm}$. posterior to the cornea. The radius of the anterior surface of the lens is $10 \mathrm{~mm}$. Find therefore on the optical axis a point $3.6+10=13.6 \mathrm{~mm}$. behind the cornea. From this point as a centre describe an are with a radius of $10 \mathrm{~mm}$. that shall intersect the optical axis $3.6 \mathrm{~mm}$. behind the cornea. A segment of this arc will represent the anterior surface of the lens. 
The thickness of the lens, accommodated for distant objects, is $3.6 \mathrm{~mm}$. Mark this point. Here the posterior surface of the lens intersects the optical axis. The radius of the posterior surface is $6 \mathrm{~mm}$. Find therefore a point on the optical axis $6 \mathrm{~mm}$. in front of the posterior surface of the lens. With this point as a centre describe with a radius of $6 \mathrm{~mm}$. the segment of the are that shall represent the posterior surface of the lens. Mark upon this drawing the cardinal points of the lens, as follows.

Optical Centre. - In the cornea, a simple spherical surface, rays directed to the centre of curvature, $k$, were found to pass through the refracting surface unchanged in direction. In thin convex lenses having a long focal distance it is generally assumed that any ray passing through a point within the lens termed the optical centre, $o$, is not refracted. In thick lenses, on the contrary, every ray excepting that coinciding with the principal axis is refracted (see Nodal Points).

The optical centre is situated in the principal axis within the lens. In a lens bounded on both sides by media of equal refracting power, for example, the crystalline lens bounded by the aqueous and vitreous humors, the optical centre is found by dividing the axis of the lens, $i$.e., the distance between the refracting surfaces on 
the principal axis, into two parts proportionate to the radii of the refracting surfaces.

Then

$$
10+6: 3.6=6: x
$$

$x=1.35 \mathrm{~mm}$., the distance of $o$ from the posterior refracting surface.

Then

$$
3.6-1.35=2.25 \mathrm{~mm} \text {, }
$$

the distance of $o$ from the anterior refracting surface.

Nodal Points. - In thick lenses (such as the crystalline) with short focal distance, all the rays except that which coincides with the principal axis are refracted at one or both of the spherical surfaces. In order to determine the path of rays passing through the lens it is necessary to find the nodal points. These are two points so placed that a ray directed to the first point appears on leaving the lens to have come from the second point, in a direction parallel to the entering ray. All rays coming from the optical centre, $o$, to the anterior refracting surface will after refraction appear to have come from the first nodal point, $K_{1}$, situated within the lens on the principal axis, between $o$ and $h_{1}$. Similarly, all rays from $o$ to the posterior refracting surface will appear to have come from the second nodal point, $K_{2}$, situ- 
ated between $o$ and $h_{2}$. Thus $o$ and $K_{1}$ are conjugate foci for the surface $h_{1}$, and $o$ and $K_{2}$ are conjugate foci for the surface $h_{2} . \quad K_{1}$ and $K_{2}$ are virtual images of $o$; that is, if $o$ were observed through the surface $h_{1}$ the image would appear to be $K_{1}$, while if $o$ were observed through $h_{2}$ the image would appear to be $K_{2}$. As the nodal points are images of the same point $o$, they must therefore be images of each other.

The first nodal point, $K_{1}$, which is the virtual image of the optical centre, $o$, formed by rays passing from $o$ through the anterior refracting surface, $h_{1}$, and which lies at the conjugate focus of $o$, is situated $2.126 \mathrm{~mm}$. behind the anterior surface of the lens (accommodated for distant objects). The second nodal point, $K_{2}$, the virtual image of $o$ formed by rays passing from $o$ through the posterior refracting surface, $h_{2}$, is situated $1.276 \mathrm{~mm}$. in front of the posterior surface of the lens (accommorlated for distant objects). The distance between the two nodal points is $0.198 \mathrm{~mm}$.

Principal Surfaces. - Within a double convex lens are two parallel planes, termed the principal surfaces. They are perpendicular to the principal axis and are so placed that the emerging ray appears to come from a point in the second principal surface that exactly corresponds to the point in the first principal surface to which the 
entering ray is directed. Thus the point in the second principal surface from which the emergent ray appears to come is the same distance from the axis as the corresponding point in the first principal surface to which the entering ray appears to pass. In short, each principal surface is the image of the other, and is of equal size.

To determine the position of the principal surfaces there must be found between the two refracting surfaces a point, $s$, at which an object will form similar images with each refracting surface. These images being similar are images of each other and of equal size. The planes in which they lie are the principal surfaces.

The Point s. - The point $s$ lies between the two refracting surfaces at distances proportional to the principal focal distance of each. It will be remembered that the point $o$ was found by dividing the distance between the two refracting surfaces into two parts, proportional to the radii of curvature of the two surfaces. In System B the two refracting surfaces are the anterior and posterior surfaces of the crystalline lens, which is bounded by the aqueous and vitreous humors, media of equal refractive power. The focal distances of the refracting surfaces are in this case proportional to the radii of curvature. Thus the 
division of the distance between the refracting surfaces is the same for both $s$ and $o$, and therefore $s$ and $o$ coincide. In System C, on the contrary, the first medium is the air, and the last the vitreous humor. The principal focal dis tances are proportional to the coefficients of refraction of the first and last media; they are no longer proportional to the radii of curvature; therefore $s$ and $o$ no longer coincide, and their images, lying in the principal surfaces and at the nodal points, respectively, no longer coincide, but must be found separately.

Principal Points. - At the intersection of the principal surfaces with the principal axis lie the principal points, ${ }^{1} H_{1}$ and $H_{2}$. The second principal point is the image of the first. Rays which in the first medium are directed to the first principal point are directed to the second principal point in the last medium, $i$. e., after the last refraction. The anterior principal focal distance is calculated from the first principal point, and the posterior principal focal distance from the second principal point.

Principal Focal Distances. - The posterior focal distance of the lens (accommodated for distant

1 The principal points, $H_{1}$ and $H_{2}$, coincide with the nodal points, $K_{1}$ and $K_{2}$, when the first and last media of the optical system have the saine refractive power. 
objects) is $50.617 \mathrm{~mm}$. The anterior focal distance is the same, for the lens is bounded by media of equal density.

\section{Cardixal Points of the Eye (Sys'tem C)}

Examine construction drawings of System A (the cornea) and System B (the Jens). System C must be a combination of $A$ and $B$.

Note: 1. With System C as with System A the first and last media have different refractive powers. Therefore the principal points cannot coincide with the nodal points. 2. The relation between the nodal point $k$ of System $A$ and the nodal points $K_{1}$ and $K_{2}$ of System $\mathrm{B}$ is such that the nodal points of System $\mathrm{C}$ will lie near the posterior surface of the crystalline leus. 3. The principal point $h_{1}$ of System A lies on the anterior surface of the cornea, and the principal points $H_{1}$ and $H_{2}$ of 'System $\mathrm{B}$ lie in the lens. Hence those of System C must lie in the aqueous humor. 4. In System $\mathrm{C}$ the collecting power of System B is added to that of System A. The focal distances in System C will therefore be less than those of $\mathrm{A}$ or $\mathrm{B}$.

Principal Surfaces. - The principal surfaces are found from the point $s$. If a perpendicular be drawn at $s$ the image of that perpendicular formed 
by the cornea will be of equal size with the image of it formed by the crystalline lens. These similar images will lie in the principal surfaces. The image which the cornea forms of the point $s$ will be the first principal point, $H_{1}$ of System $\mathrm{C}$, and the image which the lens forms of $s$ will be the second principal point, $H_{2}$ of System C. The point $s$ lies between $h_{1}$ of System $\mathrm{A}$ and $H_{1}$ of System B, at distances proportional to the posterior focal distance $\left(F_{2}=31.095 \mathrm{~mm}\right.$.), of System $\mathrm{A}$ and the anterior focal distance $\left(F_{1}=50.617\right.$ $\mathrm{mm}$.) of System B. The distance between $h_{1}$, which lies at the anterior surface of the cornea, and $H_{1} B$, which lies $2.126 \mathrm{~mm}$. behind the anterior surface of the lens, is $3.6 \mathrm{~mm}$. (the distance between the cornea and the lens) plus $2.126 \mathrm{~mm}$. $=5.726 \mathrm{~mm}$. This distance is to be divided in the proportion $50.617: 31.095$.

$$
50.617+31.095: 31.095:: 5.726: x \text {. }
$$

$x=2.179$. Hence $s$ lies $2.179 \mathrm{~mm}$. behind the cornea, and $5.726-2.179=3.547 \mathrm{~mm}$. in front of the anterior principal point of the crystalline lens.

The first or anterior principal point of the eye, $H_{1} C$, is the virtual image of $s$ formed by the cornea; it lies at the conjugate focus of $s$, and its position is determined by the formula $(3 \mathrm{~b})$, page 444. 


$$
\begin{aligned}
F_{1} A & =23.266 \mathrm{~mm} . \\
F_{2} A & =31.095 \mathrm{~mm} . \\
f_{2} A^{*} & =2.179 \mathrm{~mm}
\end{aligned}
$$

The first principal point, $H_{1} C$, is $23.266 \times 2.179$ $\frac{31.095-2.179}{3.05} \mathrm{~mm}$. behind $h_{1}$ the anterior surface of the cornea.

The second or posterior principal point of the eye, $H_{2} C$, is the virtual image of $s$ formed by the lens. It also is found by formula $(3 \mathrm{~b})$.

$$
\begin{aligned}
F_{1} B & =50.617 \mathrm{~mm} . \\
F_{2} B & =50.617 \mathrm{~mm} . \\
f_{2} B \dagger & =3.547 \mathrm{~mm} .
\end{aligned}
$$

The second principal point, $H_{2} C$, is $50.617 \times 3.547$ $\frac{50.617 \times 3.547}{50.617-3.547}=3.814 \mathrm{~mm}$. before the posterior principal point of the lens; this point lies 5.924 mm. behind the cornea; hence $H_{2} C^{\prime}$ lies 5.924 $-3.814=2.11 \mathrm{~mm}$. behind the anterior surface of the cornea. The distance between the two principal points is $2.11-1.75=0.36 \mathrm{~mm}$.

Nodal Points. - The nodal points are virtual images of the point $o$, which divides the distance between the nodal points of System A and Sys-

* The distance of the object $s$ from the refracting surface, in this case, the cornea.

$\dagger$ The distance of the object $s$ from the anterior principal point of the crystalline lens. 
tem $\mathrm{B}$ into two parts, proportional to the anterior focal distance of the cornea $(23.266 \mathrm{~mm}$.) and the focal distance of the lens $\left(50.617 \mathrm{~mm}\right.$.). As $K_{1} A$ lies $7.829 \mathrm{~mm}$. and $K_{1} B 5.726 \mathrm{~mm}$. behind the cornea, the distance between them is $2.103 \mathrm{~mm}$. This is to be divided in the proportion $23.266: 50.617$. $23.266+50.617: 50.617:: 2.103: x . \quad x=1.4408$. Thus $o$ lies $1.440 \mathrm{~s} \mathrm{~mm}$. behind the first principal point of the crystalline lens (System B) and consequently

$$
5.726+1.4408=7.167 \mathrm{~mm} \text {. }
$$

behind the cornea.

By formula ( $3 \mathrm{~b}), K_{1} C$, the first nodal point or the image of $o$ formed by the cornea, is found to be $23.266 \times 7.167$ $\frac{23.266 \times 7.167}{31.095-7.167}=6.97 \mathrm{~mm}$. behind the cornea.

The second nodal point, $K_{2} C$, or image formed of 0 by the crystalline lens, is $\frac{50.617 \times 1.4408}{50.617-1.4408}$ $=1.401 \mathrm{~mm}$. behind the second principal point of the crystalline lens, and consequently 5.924 $+1.401=7.33 \mathrm{~mm}$. behind the cornea.

The distance $K_{1} K_{2}$ between the first and second nodal points of System $\mathrm{C}$ is $7.33-$ $6.97=0.36 \mathrm{~mm}$. The distance $H_{1} H_{2}=K_{1} K_{2}$.

Principal Foci. - Rays falling on the cornea parallel to the principal axis are refracted by $l_{1} A$ and 
converged to the point $\phi_{2} A$, situated $31.095 \mathrm{~mm}$. behind the cornea. On their way they are further refracted by System B. $H_{1} B$ is $5.726 \mathrm{~mm}$. behind the cornea. The point $\phi_{2} A$ is $31.095-5.726$ $=25.369 \mathrm{~mm}$. behind $H_{1}$. Calculated from $H_{2} B$, the posterior principal focal distance $F_{2}$ of System $\mathrm{B}$ is $50.617 \mathrm{~mm}$. The posterior focal distance of System C is calculated by the formula

$$
f_{2}=\frac{f_{1} F_{2}}{f_{1}+F_{1}} f_{2}=\frac{25.369 \times 50.617}{25.369+50.617}=16.899 \mathrm{~mm} \text {. }
$$

behind $H_{2} \quad B$, and hence $16.899+5.924=$ $22.823 \mathrm{~mm}$. behind the comea, and $22.823-$ $2.11=20.713 \mathrm{~mm}$. behind $H_{2}$ of System C. The posterior principal focal distance of the eye is therefore $20.71 \mathrm{~mm}$.

Parallel rays falling on the posterior surface of the lens are refracted by the lens and converge at a point $\phi_{1} B=50.617 \mathrm{~mm}$. in front of $H_{1} B$. They meet the anterior surface of the cornea $50.617-5.726=44.891 \mathrm{~mm}$. from $H_{1} B$. They are further converged by the cornea to

$$
\frac{23.266 \times 44.891}{31.095+44.891}=13.75 \mathrm{~mm} \text {. }
$$

before the cornea, or $13.75+1.75=15.5 \mathrm{~mm}$. before $H_{1} C$. The anterior principal focal distance of the eye is therefore $15.5 \mathrm{~mm} .^{1}$

${ }^{1}$ In this discussion I have followed closely, in some places 


\section{Calculation of the Situation and Size of Dioptric Images}

Draw perpendiculars through the optical axis of System C at the following points: the anterior principal focus, $\phi_{1} C$, the first principal point, $H_{1} C$, the second principal point, $H_{2} C$, and the posterior principal focus, $\phi_{2} C^{\prime}$ (retina). Mark on the optical axis the first and second nodal points, $K_{1} C$ and $K_{2} C$.

The following facts should be borne in mind: 1. Every ray which in the first medium is directed to the first nodal point appears in the last medium to come from the second nodal point and is parallel to its original direction. 2. The point at which the ray cuts the second principal surface is the same distance from the optical axis as the point at which the ray cuts the first principal surface; between the principal surfaces the ray is parallel to the optical axis. 3. All rays parallel in the first medium unite in one point in the second or posterior focal surface (the plane passing through the posterior principal focus vertical to the optical axis); if

almost literally, the valuable works of Domrlers (Accommodation and Refraction of the Eye, New Sydenham Society, I,ondon, 1864) and Helnholtz (Handbuch der physiologischen Optik, 2te Auflage, 1896). 
these rays be parallel to the axis they will unite in the posterior principal focus. Conversely, all rays parallel in the second medium unite in one point on the first or anterior focal surface, and if parallel to the axis they unite at the anterior principal focus (Gauss).

1. Find the course in the vitreous humor of any ray, $a b$, which enters the eye.

Draw in the first medium a ray, $a^{\prime} b^{\prime}$, parallel to $a b$, directed to the first nodal point. In the second medium draw this ray, parallel to its original direction, from the second nodal point to the posterior focal surface. Then $a b$ must also meet the posterior focal surface at this same place; for all rays parallel in the first medium converge in the second medium to one point in the posterior focal surface. Produce $a b$ to the first principal surface, thence, parallel with the optical axis, to the second principal surface, thence, through the vitreous humor, to the point already found in the posterior focal surface.

2. Let $i$ be any point in the first medium (the air). Find its image (for convenience $i$ should be placed at least $10 \mathrm{~cm}$. in front of the cornea).

Draw from $i$ a ray, $i_{1} j_{1}$, through the first and second nodal points, as directed above. Draw from $i$ a second ray, $i_{2} j_{2}$, parallel with the optical axis. This ray will cut the second focal surface 
at the principal focus. Produce $i_{2} j_{2}$ until it meets $i_{1} j_{1}$. The point of intersection will be the image of the point $i$.

\section{Reduced Eye}

The distance of less than one fourth millimetre which separates one principal point from the other is so small that it may be neglected without any error of practical importance. Thus the two principal points may be combined in one point lying $2.34 \mathrm{~mm}$. behind the anterior surface of the cornea of the normal. ${ }^{1}$ Similarly the two nodal points may be combined in one point lying $0.48 \mathrm{~mm}$. in front of the posterior surface of the lens, or about $16 \mathrm{~mm}$. in front of the retina. The nodal point $k$ of the cornea (System A) is about $14 \mathrm{~mm}$. in front of the retina. The nodal point of the lens and that of the cornea are combined in the reduced eye in a nodal point situated $15 \mathrm{~mm}$. from the retina. The lens may therefore be omitted. Indeed, the cornea is normally the principal refracting surface; its focal distance is $31.095 \mathrm{~mm}$., while that of the crystalline lens is $50.617 \mathrm{~mm}$.; if the lens were not present, parallel rays entering the eye would be focussed by the

1 Listing: Wagner's Handwörterbuch der Physiologie, 1853, iv., p. 495 . 
cornea in a point about $10 \mathrm{~mm}$. behind the retina. Thus the eye is reduced to a single refracting surface, the cornea, separating two media, the air and the vitreous humor. The index of refraction of these media is $\frac{4}{3}$. The principal focal distances are proportional to the coefficients of refraction of the first and last media; $F_{1}$ is $15 \mathrm{~mm}$. and $F_{2}$ $20 \mathrm{~mm}$., measured from the principal point. The visual axis (from the cornea to the retina of the reduced eye) is therefore $20 \mathrm{~mm}$. In order to bring parallel rays to a focus at $20 \mathrm{~mm}$., the index of refraction being $\frac{4}{3}$, the radius of curvature of the cornea of the reduced eye should be $5 \mathrm{~mm}$.

In such a reduced eye the retinal images have the same position and size as in the ordinary eye. The reduced eye is shown in normal size in Fig. 65.

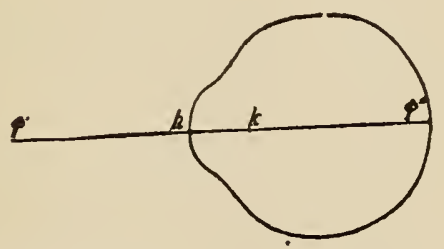

Fig. 65. The reduced eye. Normal size (Donders).

$K$ is the optical centre or nodal point.

$h$, the principal point.

$K h=5 \mathrm{~mm}$., the radius of curvature of the refracting surface. 
$\phi_{1}$, the anterior principal focus, - the focus of rays parallel in the vitreous.

$\phi_{2}$, the posterior principal focus, - the focus of rays parallel in the air.

$h \phi_{1}=F_{1}$, the anterior focal distance, $=15 \mathrm{~mm}$.

$h \phi_{2}=F_{2}$, the posterior focal distance, $=20 \mathrm{~mm}$.

$$
\frac{n_{2}}{n_{1}}=\frac{4}{3}=\frac{F_{2}^{\prime}}{F_{1}}=\frac{20}{15} .
$$

With the reduced eye many calculations may be rapidly and easily performed. 


\section{AVERAGE MEASUREMENTS OF NORMAL (EMMETROPIC) EYE $^{1}$}

Diameter of cornea .

Mean diameter of pupil

Thickness of cornea . . . . . . . . . . 1

Thickness of lens accommodated for near objects

Thickness of lens accommodated for distant objects . . . . . . . .

Thickness of retina at fundus . . . . .

Distance between retinal ressels and rod and cone layer

Diameter of optic disk $: \quad . \quad . \quad . \quad$.

Diameter of yellow spot . . . . . . .

Diameter of forea centralis . . . . .

Diameter of cone in forea . . . . . .

Diameier of rod . . . . . . . . .

$\mathrm{mm}$.

\section{4}

4

3.6

$0.2-0.3$

$0.2-0.3$

1.5

1.25

0.22

$0.004-0.005$

0.0018

\begin{tabular}{|c|c|c|}
\hline & \multicolumn{2}{|c|}{ Accommodated for } \\
\hline & $\begin{array}{l}\text { Distant } \\
\text { objects. }\end{array}$ & $\begin{array}{c}\text { Near } \\
\text { objects. }\end{array}$ \\
\hline $\begin{array}{l}\text { Refractive index of aqueous } \\
\text { and vitreous humors } \\
\text { Total refractive index of crys- } \\
\text { talline leus } \\
\text { Radius of curvature of cornea. } \\
\text { Radius of curvature of aute- } \\
\text { rior surface of lens. } \\
\text { Radius of curvature of poste- } \\
\text { rior surface of lens }\end{array}$ & $\begin{array}{l}1.3365 \\
1.4371 \\
7.829 \\
10.00 \\
6.0\end{array}$ & $\begin{array}{l}1.3365 \\
1.4371 \\
7.829 \\
6.0 \\
5.5\end{array}$ \\
\hline
\end{tabular}

1 It should be understood that the figures given in this table are the mean of numerous obserrations. The variation in different eyes is considerable, though in most cases not great enough to be of practical importance. 


\begin{tabular}{|c|c|c|}
\hline & \multicolumn{2}{|c|}{ Accommodated for } \\
\hline & $\begin{array}{l}\text { Distant } \\
\text { objects. }\end{array}$ & $\begin{array}{c}\text { Near } \\
\text { objects. }\end{array}$ \\
\hline $\begin{array}{l}\text { Distance from anterior surface } \\
\text { of cornea to anterior sur- } \\
\text { face of lens }\end{array}$ & 3.6 & 3.2 \\
\hline $\begin{array}{l}\text { Distance from anterior surface } \\
\text { of cornea to posterior sur- } \\
\text { face of leus }\end{array}$ & 7.2 & 7.2 \\
\hline \multicolumn{3}{|l|}{ Calculated } \\
\hline $\begin{array}{l}\text { Anterior principal focal dis- } \\
\text { tance of cornea }\end{array}$ & 23.266 & 23.266 \\
\hline $\begin{array}{l}\text { Posterior principal focal dis- } \\
\text { tance of cornea }\end{array}$ & 31.095 & 31.095 \\
\hline $\begin{array}{l}\text { Anterior and posterior princi- } \\
\text { pal focal distance of lens } \\
\text { Distance of anterior principal }\end{array}$ & 50.617 & 39.073 \\
\hline $\begin{array}{l}\text { point of lens from auterior } \\
\text { surface of lens }\end{array}$ & 2.126 & 1.989 \\
\hline $\begin{array}{l}\text { Distance of posterior principal } \\
\text { point of lens froin poste- } \\
\text { rior surface of lens. }\end{array}$ & -1.276 & -1.823 \\
\hline $\begin{array}{l}\text { Distance of the two principal } \\
\text { points of lens from each }\end{array}$ & & 0188 \\
\hline Posterior principal focal dis- & 0.198 & \\
\hline $\begin{array}{c}\text { tance of eve } \\
\text { Anterior principal focal dis- }\end{array}$ & 20.713 & 18.689 \\
\hline tance of eye . . . & 15.498 & 13.990 \\
\hline $\begin{array}{l}\text { Distance from anterior sur- } \\
\text { face of cornea to }\end{array}$ & & \\
\hline First principal point & 1.753 & 1.858 \\
\hline Second principal point . & 2.106 & 2.257 \\
\hline First nodal point . . & 6.968 & 6.566 \\
\hline Second nodal point . . & 7.321 & 6.965 \\
\hline Anterior principal focus & -13.745 & -12.132 \\
\hline Posterior principal focus & 22.819 & 20.955 \\
\hline Distance upon optical axis & & \\
\hline $\begin{array}{l}\text { from anterior surface of } \\
\text { cornea to retina }\end{array}$ & 23.0 & 23.0 \\
\hline
\end{tabular}

In accommodation a clear image of an object $152 \mathrm{~mm}$. in front of the cornea, or $140 \mathrm{~mm}$. in front of the anterior principal focus, will be formed upon the retina. 


\section{Relations of the Visual Axis}

It has already been stated that the refracting surfaces of the eye are centred, often imperfectly, upon a right line, the optical axis. This line normally meets the retina between the yellow spot and the optic papilla or exit of the optic nerve. To see a luminous point clearly, the image of the point must fall on the centre of the yellow spot. The line passing from the centre of the yellow spot through the nodal point to the luminous point is termed the visual axis. Unless the luminous point already lie in the visual axis, it must for distinct vision be brought there by the rotation of the eyeball. The object is then said to be "fixed" by the eye. The point about which the eye rotates is the centre of rotation. The line between the luminous point and the centre of rotation is the line of fixation.

The line of fixation and the visual axis should nearly coincide. Generally, the visual axis and the optical axis do not coincide. In other words, the visual axis is generally a secondary axis, and the planes of the refracting surfaces are oblique to it. The optical axis passes to the inner side of the yellow spot. It intersects the visual axis at the nodal point. Hence 
the nodal point becomes the vertex of an angle, the angle gamma, $\gamma$, the legs of which are the anterior portion of the optical and visual axes. The angle $\gamma$ usually reaches $5^{\circ}$, but may reach $10^{\circ}$.

In emmetropia and hypermetropia, the visual axis passes through the cornea on the inner side of the optical axis; angle $\gamma$ is then positive. The eyeball must rotate outwards in order to fix an object. Thus the visual axes seem to diverge. Hence the angle $\gamma$ must be considered in estimating the degree of a divergent squint.

In myopia, the visual axis may coincide with the optical axis or pass through the cornea on the outer side of the optical axis. In the latter case, angle $\gamma$ is negative. In this condition the eyeball must rotate inwards in order to fix the object. The deviation inwards may be confused with convergent squint.

Draw a diagram showing angle $\gamma$.

Visual Angle. - Draw an arrow in front of a diagram of the reduced eye. Draw lines from the nodal point through and beyond the two extremities of the object.

The angle included between the lines drawn from the nodal point to the extremities of the object is termed the visual angle.

Apparent Size. - Within the lines marking the 
visual angle draw a second arrow parallel to the first and twice its distance from the nodal point. Produce the visual lines from the nodal point to the retina.

Observe that the retinal images of the large and the small arrow are of equal size. The two objects subtend the same visual angle. Thus the apparent size of an object depends upon the visual angle.

Size of Retinal Image. - In the emmetropic eye (the eye accommodated for distant vision) the size of the object $B$ is to the size of the retinal image $b$ as the distance from the object to the nodal point of the reduced eye, $g_{1}$, is to the distance from the nodal point to the retina, $g_{2}$.

$$
B: b:: g_{1}: g_{2}
$$

The retinal image is smaller than the object by the number of times $g_{2}=15 \mathrm{~mm}$. is contained in the distance, in millimetres, of the object from the nodal point.

Calculate the size of the retinal image of a post one metre high placed 300 metres from the observer's eye.

Acuteness of Vision. - Draw upon the visual axis of the reduced eye a series of arrows of equal size, each bisected by the axis. Draw lines from 
the extremities of these arrows to the nodal point.

Observe that as the object recedes from the eye the visual angle and the retinal image become smaller. When the visual angle is less than one minute, the retinal image will be too small to be perceived; the limit of perception will be reached.

Smallest Perceptible Image. - On a black card gum one millimetre apart, and parallel with each other, two slips of white paper one millimetre in width. Place the card about six metres in front of a window or other sufficient light. Face the card and move backward until the millimetre space between the two white slips disappears because the slips can no longer be seen separately. Measure the distance $g_{1}$ from the object to the nodal point. Calculate the size of the retinal image (formula 5). Compare this result with the diameter of the cones in the region of distinct vision (page 461).

Measurement of Visual Acuteness. - Taking $1^{\prime}$ as the average smallest visual angle at which an object is perceptible, Snellen built up a set of test letters by combining small squares each of which subtends an angle of $1^{\prime}$. Thus the lines of which the letters are formed subtend an angle of $1^{\prime}$. The spaces between the lines also subtend 
this angle. Only such letters are used as can be drawn approximately within a square that shall contain twenty-five of the smaller squares, and shall subtend an angle of $5^{\prime}$. Thus the strokes and, so far as possible, the spaces between the strokes are one fifth the size of the letter. The size of the letter the perception of which constitutes normal vision at a given distance (that is, the letter that subtends a visual angle of $5^{\prime}$ at the given distance) is obtained by multiplying the distance by $0.001454 \mathrm{~mm}$., which is the tangent ${ }^{1}$ of the angle of $5^{\prime}$. At the distance of one metre the size of the standard letter is $1000 \times 0.001454=1.45 \mathrm{~mm}$. Near each of Snellen's test letters is recorded the distance viewed from which the letter will subtend a visual angle of $5^{\prime}$ in the emmetropic eye.

As some of the letters are not easily recognized by the astigmatic eye ( $D$, for example, being sometimes mistaken for $B$ ), the acuteness of vision should not be pronounced normal unless each letter of the entire series can be read at the dis-

1 To obtain the tangent of an angle draw a circle with the vertex of the angle as the centre. The two legs of the angle are radii of the circle. Draw a perpendicular (tangent line) from the end of one radius to the prolongation of the other: Divide the length of the perpendicular by the length of the radius; the quotient is the function called the tangent of the angle- 
tance corresponding to the number of the series. The acuteness of vision is expressed by $\frac{d}{D}$; where $d$ is the greatest distance at which the letters in any line are seen distinctly by the eye examined, and $D$ the distance at which they can be seen by the normally acute eye.

Place the subject in a well-lighted room six metres (approximately 20 feet) in front of a card of Snellen's test types. Rays from an object six metres distant are practically parallel. At this distance the letters numbered VI should be read. If they are clearly visible, $V=\frac{6}{6}$; acuteness of vision is normal. If the subject at 6 metres cannot see distinctly letters larger than those marked XVIII metres (approximately 60 feet), $\mathrm{V}=\frac{6}{18}$; acuteness of vision is one third the normal.

In some eyes vision is so acute that types constructed with a visual angle of 4 minutes ( $\frac{5}{4}$ the normal angle) can be seen clearly. 


\section{ACCOMMODATION}

Accommodation. - Look at any distant object. The object will be seen clearly. The (practically) parallel rays proceeding from the object are brought to a focus on the retina.

Look at an object ten inches from the eye.

The rays proceeding from this object are evidently divergent, yet the object is seen clearly. The divergent rays have also been focussed on the retina. This power of voluntarily bringing divergent rays to a focus on the retina is termed accommodation.

Scheiner's Experiment. - With a fine needle pierce in a card two holes at a distance from each other a little less than the diameter of the pupil (average $4 \mathrm{~mm}$.). Hold the card with the holes horizontal and near the pupil. Look through the holes at a pin or needle held vertical about $15 \mathrm{~cm}$. (6 inches) in front of the eye.

The needle will be seen clearly.

Move the index finger over one of the holes.

There will be no change except that the visual field will be darker.

Fix a distant object, for example, a cloud.

The needle will appear double, and each image will be rendered indistinct by dispersion circles.

Move the index finger orer the left-hand hole. 
The right-hand image will disappear.

Hold the needle about $100 \mathrm{~cm}$. away and fix some nearer object. The needle will appear double. Close the left-hand hole.

The left-hand image will disappear.

Draw a diagram to explain these observations. Remember that a separate image of the needle will be formed by the rays passing through each hole in the card.

Dispersion Circles. - Place a printed page about two feet in front of one eye, and shut the other eye. Observe the letters through a piece of wire gauze held six inches in front of the eye.

Either the wire or the letters can be distinctly seen, but not both at once. If the letters are seen clearly, each wire will appear as a broad indistinct line made up of superposed dispersion circles and vice versa.

Diameter of Circles of Dispersion. - 1. If the eye be accommodated for objects at an infinite distance (practically twelve metres or more), the image of a near object will fall behind the retina. The imagre will lie in the conjugate focus of the olject, and the position of the image can be calculated by the formula for conjugate foci (page 444). From this formula may be derived

$$
y=\frac{F_{2} F_{1}}{g}
$$


$y=f_{2}-F_{2}$, the distance from the retina to the image behind it.

$g$, the distance from the anterior focus $\phi_{1}$ to the object.

$\phi_{1}$ lies $20 \mathrm{~mm}$. from the nodal point $K$.

$F_{2} F_{1}$, in the reduced eye, is $20 \times 15=300 \mathrm{~mm}$. from $K$.

Find the distance behind the retina of an image whose object is $320 \mathrm{~mm}$. from $K$.

The distance is $1 \mathrm{~mm}$.

2. If $y$ is known, the diameter of the dispersion circles can be calculated. In the example just given, the pencil of rays diverging from each luminous point in the object was reunited in a single point one millimetre behind the retina. At the retina, the converging cone had a certain section, i.e. the circle of dispersion. The base of the cone is evidently the pupil, which in the reduced eye is taken to be $19 \mathrm{~mm}$. in front of the retina and $4 \mathrm{~mm}$. in diameter. ${ }^{1}$

The length of $y$ divided by the length of the whole cone $(19 \mathrm{~mm}$.$) , gives the proportion in$

1 The diameter of the cone is not precisely that of the pupil. The rays in the vitreous would appear to come from the image of the pupil formed by the lens. Thus the diameter changes from 4 to $4.23 \mathrm{~mm}$. At the same time the position of the base is changed from $3.6 \mathrm{~mm}$. (the distance of the plane of the pupil behind the cornea) to 3.7 behind the cornea. This brings the base of the cone $19 \mathrm{~mm}$. in front of the retina, which is the position assumed for it in the reduced eye. 
which the diameter of the cone at its base ( $4 \mathrm{~mm}$.) is reduced at the retina. In the example taken $y=1 \mathrm{~mm}$. Then the proportion sought is $1: 19+1=\frac{1}{20}$. Thus the diameter of the dispersion circle is $\frac{1}{20}$ of $4 \mathrm{~mm} .=\frac{1}{5} \mathrm{~mm}$.

3. Calculate the size of the dispersion circle produced by an object twelve metres from the emmetropic eye. At this distance the dispersion circles are so small as to cause no perceptible lack of clearness in the image.

Accommodation Line. - Hold a needle two inches from a printed page. Bring the eyes as near the needle as is possible without causing the image of the needle to blur. When the needle is at this "near point" of accommodation it will be seen clearly, but the printed words will be indistinct. Draw back the eyes gradually.

Soon a point will be reached at which both needle and print will be seen distinctly. The greatest distance between two objects on the visual line at which the two may both be seen clearly while the eye is accommodated for either, is called the accommodation line. The length of the accommodation line increases as the object is removed from the eye. 


\section{Mechanisy of Accommodation}

Narrowing of Pupil. - 1. Watch the pupil while the subject accommodates first for a distant and then for a near object.

In accommodation for near objects the pupil contracts.

2. Hold a pencil about thirty centimetres in front of one eye. Close the other eye. The pencil is seen clearly. More the pencil towards

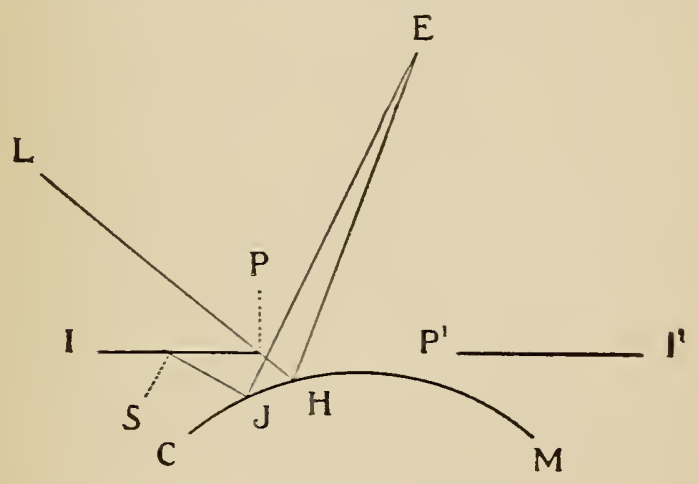

Fig. 66 .

the eye until its image becomes indistinct from dispersion circles. Now observe the pencil through a pin-hole in a card placed in front of the pupil.

The image is sharper. The size of the dispersion circles is diminished by making the apert:ure smaller and thus cutting off the rays 
that meet the refracting surfaces at a distance from the optical axis (compare page 434).

Relation of Iris to Lens. - 1. Stand the convex mirror upright on a level with the eye of the observer. Over the mirror (Fig. 66, CM) place a diaphragm of black paper, $\mathrm{II}^{\prime}$, with an aperture, $\mathrm{PP}^{\prime}$, four millimetres in diameter. Let this aperture be the pupil and the convex mirror be the crystalline lens. The wooden block in which the mirror is held will support the diaphragm so that there will be a space between the border of the pupil and the surface of the mirror. Let the lamp, L, be on one side of the aperture and the observer's eye, E, on the other. By means of the convex lens of $6.5 \mathrm{~cm}$. focal distance furnished with the ophthalmoscope concentrate the light upon the margin of the pupil in the direction LH. It will pass the margin $\mathrm{P}$ and be reflected from the mirror to the eye in the direction H E. No rays from $\mathrm{L}$ can reach the mirror between $\mathrm{H}$ and $\mathrm{C}$. This portion of the mirror will reflect the posterior side of the diaphragm. Thus the light from $S$ falling on the mirror at $J$ will he reflected in the direction $J \mathrm{E}$ to the observer's eye, and a dark band, the image of the back of the diaphragm, will appear in the mirror between the inage of $\mathrm{L}$ at $\mathrm{H}$ and the margin of the p"ipil. 
Depress the paper diaphragm until the margin of the pupil lies against the mirror. The black line will disappear, because the ray $\mathrm{J} \mathrm{E}$, reflected from the back of the diaphragm, is intercepted. The space $\mathrm{PH}$ is closed (Helmholtz).

2. In a dark room repeat Experiment 1 upon the eye. The iris will be the diaphragm, I I', and the anterior surface of the crystalline lens will be the convex mirror. The light and the observer's eye should be placed as in Fig. 66 .

The bright image of the light formed by the cornea, should be neglected. Near this image are two others, very much fainter. The larger of the two is indistinct and upright; it is reflected from the anterior surface of the crystalline lens. The smaller is a sharp, inverted image from the posterior surface of the lens. By moving the glass lens the light may be thrown at will on all parts of the border of the pupil.

No dark line or image of the posterior surface of the iris will be seen. The margin of the iris lies upon the lens.

Changes in the Lens. -1 . Direct the subject to cover one eye. Place a needle at the near point of the other eye in line with some distant object that can be clearly seen. The two objects must be kept accurately in line throughont the experiment. Let the observer stand at one side of and 
a little behind the subject, so that he shall see about half of the corneal image of the black pupil of the subject's eye projecting beyond the corneal border of the sclera. Note, from within outwards, the optical section or profile of the margin of the sclera, the anterior half of the pupil, a clear portion of the cornea, and finally a dark stripe which is the most anterior portion of the cornea. ${ }^{1}$

Watch carefully the clear interval between this dark stripe and the profile of the pupil while the subject, keeping the eye steadily in one position, accommodates first for the distant and then for the near object.

The interval between the corneal stripe and the border of the pupil diminishes on acconmodation for near objects. Hence the border of the pupil moves forward. If this were not the case, the interval would become larger, for the pupil narrows in accommodation. Accidental turning of the subject's eye towards the observer would also cause the interval to appear larger. As the margin of the iris lies upon the lens, this observation is evidence that the anterior surface of the

1 The sclera projects over the iris. The inner surface of the projecting portion is in sharlow. The profile view of the image formed of this projecting portion by the refraction of the cornea is the dark line observed in the alove experiment. It is dark by contrast with the image of the well-lighted iris. 
lens moves forward in accommodation (Helmholtz).

2. Place the diaphragm with L-shaped aperture in the lantern. In a dark room place the lantern in front and to the inner side of the subject's eye, so that the rays shall make an angle of about $40^{\circ}$ with the visual axis of the eye directed forwards. Let the observer's eye be in a corresponding position to the outer side of the subject's eye. In the visual axis of the subject's eye place an object at the near point and one at the far point (six metres). Let the subject accommodate for the far point.

Note the sharp, very bright, upright image reflected from the cornea, the indistinct, faint, upright, slightly larger image from the convex anterior surface of the crystalline lens, and lastly, the sharper, faint, inverted, small image reflected from the concave posterior surface of the lens. ${ }^{1}$ The image from the anterior surface of the lens lies apparently $8-12 \mathrm{~mm}$. behind the pupil, and therefore disappears behind the border of the iris upon slight changes in the position of the light or the observer's eye. The image from the posterior surface lies apparently about $1 \mathrm{~mm}$.

1 These images may he masnified with aclvantage by looking at them through the lens of $7.5 \mathrm{~cm}$. focal distance furnished with the ophthalmoscope. 
behind the pupil, and therefore is not much displaced towards the pupil and the corneal image upon slight movements of the light or the observer's eye.

Let the subject accommodate for the near point.

The image from the anterior surface of the lens will become considerably smaller, and usually it will approach the middle of the pupil. The image formed by a convex mirror becomes smaller the smaller the radius. Hence in accommodation the anterior surface of the lens becomes more convex. ${ }^{1}$

The image from the posterior surface also becomes smaller, but the change is too slight to be observed by the method employed in this experiment. Some diminution in size would be expected from the shifting of the cardinal points in accommodation. Exact measurements with the ophthalmometer show that the change is too great to be explained in this way.

Thus in accommodation the focal distance of the lens is shortened and its principal points move forwards.

1 If in accommodation the anterior surface approached the cornea, the image would become smaller through refraction in the cornea, even though the anterior surface did not become more convex. Calculation shows that the change thus produced is very small relative to that actually observed in the above experiment. 


\section{Measurement of Accommodation}

Far Point. - The most distant point of which the eye at rest, $i$. $e$. the ciliary muscle entirely relaxed, can form a clear image on the retina was termed by Donders the far point (punctum remotum $=r)$. The distance of $r$ from the eye $=R$. In the emmetropic eye parallel rays are brought to a focus on the retina; $r$ is theoretically at an infinite distance. Practically, if the accommodation be kept at rest by voluntarily relaxing the ciliary muscle or by paralyzing the innervation of the muscle with atropine, the far point will be found at twelve metres, at which distance objects produce dispersion circles so small as to cause no perceptible lack of clearness in the image.

In the myopic eye, $r$ is a short distance in front of the eye.

In hypermetropia, only convergent rays can be focussed on the retina of the eye at rest. Parallel and divergent rays can be focussed only by use of the accommodation mechanism; $r$ is therefore negative.

Determination of Far Point. - Place the subject in a well-lighted room six metres in front of a card of Snellen's test-types. At this distance the 
normal eye can read the letters numbered VI. If the subject sees these letters clearly the acuteness of vision is normal, and $R$ is infinite. If the subject reads I at one metre, II at two metres, but cannot read VI at six metres, bring the test card towards the eye until a point is reached at which the letters numbered VI are seen clearly. This is the far point.

Near Point. - 1. Look through the holes in the card used for Scheiner's experiment at a needle placed vertically about $30 \mathrm{~cm}$. (twelve inches) in front of one eye. Obtain a single clear image of the needle. Bring the needle nearer the eye. As the distance between the needle and the eye becomes shorter, the rays proceeding from the needle become more divergent and require a greater convexity of the lens to bring them to a focus on the retina. A point will be reached at which the divergence exceeds the utmost converging power of the dioptric apparatus and the images received through the two holes in the card can no longer be made to coincide on the retina; the needle will then appear double. This is the near point of accommodation ( punctum proximum $=p$ ). The distance from $p$ to the eye $=P$.

Determination of Near Point. - Hold in front of the eye a test card containing print so small that 
it shall subtend the standard angle of $5^{\prime}$ when placed $25 \mathrm{~cm}$. from the cornea. The distance from the eye at which this type can be read clearly $=P$.

Range of Accommodation. - The range of accommodation is the expression of the total accommodative power of the eye. With the eye at rest rays diverging from the far point $r$ are brought to a focus on the retina. With the ciliary muscle fully contracted, rays diverging from the near point $p$ are focussed on the retina. To bring the more divergent rays from $p$ to the same focal plane as the less divergent rays from $r$, an auxiliary lens must be employed, as in the following experiment.

Place the diaphragm with L-shaped aperture in front of the condenser. Remove the tubes holding the projecting lenses. Rays will now diverge from the illuminated $L$. Place this illuminated object at a convenient far point, for example, $26 \mathrm{~cm}$. in front of the convex lens of $10 \mathrm{~cm}$. focal length. Place a screen at the conjugate focus. Note the clear image. Move the object $8 \mathrm{~cm}$. nearer the lens. Let this be the near point. The conjugate focus now falls behind the screen and the image is blurred by dispersion circles. Place in front of the $10 \mathrm{D}$ lens an auxiliary lens of +2 D. The image will be clear. The rays diverg- 
ing from the near point will be united by the two lenses in the same focal plane in which the rays diverging from the far point were united by the first lens. The second lens has "accommodated" the optical system to the distance $R-P$.

In this experiment the power of the $+2 \mathrm{D}$ lens represents the distance $R-P$, or range of accommodation. The power of a lens is inversely proportional to its focal distance $A$. Consequently, the range of accommodation ${ }^{1}=1: A$ or $\frac{1}{A}$. Then

$$
\frac{1}{A}=\frac{1}{P}-\frac{1}{R}
$$

In the eye the auxiliary lens necessary for focussing the rays diverging from the near point is provided by an increase in the convexity of the crystalline lens. The difference in refractive power of the two lenses (the crystalline in its least convex form and the crystalline in its most convex form) is the measure of the range of accommodation of the eye. If the lens remain in its least convex form, an auxiliary lens must be placed before the cornea in order to bring rays diverging from an object at the near point to a focus on the retina. The strength of this auxiliary lens becomes then the practical measure of

1 When $1=1$ inetre. 
the range of accommodation, and $\frac{1}{A}=\frac{1}{P}-\frac{1}{R}$ becomes its numerical expression. ${ }^{1}$

In myopia $P$ may be $10 \mathrm{~cm}$. and $R 25 \mathrm{~cm}$. Then $\frac{1}{P}=\frac{100}{10}=10 \mathrm{D}, \frac{1}{R}=\frac{100}{25}=4 \mathrm{D}$. The myopia is of 4 dioptres. $\frac{1}{A}=10 \mathrm{D}-4 \mathrm{D}=6 \mathrm{D}$. In this case $P$ is greater than $A$.

In hypermetropia $P$ may be $50 \mathrm{~cm}$. and $R$ negative, $-25 \mathrm{~cm}$. Then $\frac{1}{P}=\frac{100}{50}=2 \mathrm{D}$, and $\frac{1}{R}=\frac{100}{-25}$ $=-4 \mathrm{D}$. The hypermetropia is of $4 \mathrm{D} \cdot \frac{1}{A}=$ $2-(-4)=2+4=6 \mathrm{D}$, which is the sum of $P$ and $R$.

In emmetropia $P$ may be $20 \mathrm{~cm}$. and $R$ infinite. Then $\frac{1}{A}=\frac{1}{P} . \quad \frac{1}{P}=\frac{100}{20}=5$ D. In

1 Theoretically the auxiliary lens should be placed in the eye and not in front of it, and its second nodal point should coincide with the first nodal point of the eye. The placing of the auxiliary lens in front of the cornea alters the position of the cardinal points of the combined system, and also alters the focal distance of the auxiliary lens. Put the actual changes in the lens during accommodation are nearly proportional to $\frac{1}{A}=\frac{1}{P}-\frac{1}{R}$, so that the formula serves practically for proportional magnitudes, i. c., for comparing reciprocally the different values of the range of accommodation under different circumstances. 
other words, a convex lens of $5 \mathrm{D}$ must be placed before the cornea in order to enable the eye with ciliary muscle relaxed to see clearly an object situated at the near point.

The near point recedes as the lens becomes harder with advancing age until about the seventieth year, when $R=$ infinity, and accommodation is lost.

RANGE OF ACCOMMODATION AT DIFFERENT AGES

\begin{tabular}{|c|c|c|}
\hline $\begin{array}{c}\text { Age } \\
\text { in years. }\end{array}$ & $\begin{array}{c}P \\
\text { in dioptres. }\end{array}$ & $\begin{array}{c}p \\
\text { in cm. }\end{array}$ \\
\cline { 1 - 3 } 15 & 12.0 & 8.3 \\
25 & 8.5 & 12 \\
35 & 5.5 & 18 \\
45 & 3.5 & 28 \\
55 & 1.75 & 55 \\
65 & 0.75 & 133 \\
70 & 0 & $\infty$ \\
\hline
\end{tabular}

OPHTHALMOSCOPY

Reflection from Retina. - 1. Copy the construction used to find the image of the point $i$ formed by the dioptric system C (page 457). Assume that the image is itself luminous, and that rays 
in the last medium are passing from the image to the second (now the first) refracting surface. Find the point at which these rays unite in the first medium (now the second).

The rays will unite at the original luminous point. If the eye be accommodated for a light placed in front of it, an image of the light will be formed upon the retina. A portion of the light rays entering the eye will be reflected from this image. Passing back over their original course, they will form in turn an image which will exactly coincide with the luminous object.

2. Draw a horizontal line as a visual axis. Upon this visual axis draw two reduced eyes, normal size (Fig. 65), facing each other a convenient distance apart $(5 \mathrm{~cm}$.). Let the left be the observer's eye, and the right the eye of the subject. On the visual axis behind the observer's eye draw a lamp flame. Assume that the subject's eye is accommodated for this flame.

The construction shows that were the observer's eye away, an image of the flame would be formed on the retina of the subject's eye. The image would reflect light toward the flame. This reflected light would enter by the observer's eye, and the illuminated area of the subject's retina thus be made visible, were it not that the observer's eye is necessarily placed in the visual 
axis, and thus intercepts the rays from the source of light to the subject's eye. The interior of the eye is therefore not illuminated, and the pupil remains dark.

3. Three millimetres behind the principal point of each of the two reduced eyes draw a diaphragm (iris) with an aperture (pupil) four millimetres in diameter. Assume that the subject's eye is accommodated for the pupil of the observer's eye.

Note that a dark image of the pupil of the observer's eye will be formed on the retina of the subject's eye. The rays reflected from this image will form a second dark image which will exactly coincide with the pupil of the observer's eye. Thus the observer will see only the reflection of his own black pupil in the subject's eye.

4. Throw light into the subject's pupil from a lamp held as near the observer's eye as possible. The subject should not look at either the observer or the light, and his eye should be accommodated for a distance much less or much greater than that of the observer or the light.

Part of the pupil will appear red. It has been shown in Constructions 2 and 3 that the pupil ordinarily appears black. When, however, a part of the imarge of the light on the retina of the subject cuincides with that of the pupil of the observer, 
and when the subject's eye is not accommodated for either the light or the observer's pupil, some of the light reflected from the subject's retina will reach the retina of the observer (Helmholtz).

\section{Influence of Angle between Light and Visual} Axis. - 1. Draw a reduced eye with pupil of four millimetres diameter as described above. Draw to the margins of the pupil an illuminating pencil of parallel rays that shall make with the visual axis an angle of about $20^{\circ}$. Draw the course of these rays from the pupil to the retina (see page 457). On the opposite side of the visual axis mark the nodal point of the observer's eye in such a position that the observer's visual axis shall make also an angle of about $20^{\circ}$ with the visual axis of the subject's eye. Draw rays from this nodal point to the pupil, and thence to their focus on the retina.

The portion of the interior of the eye visible to the observer will be that included between the outermost rays of the two conical pencils, the comnon base of which is the pupil. Note that the apex of the cone is a short distance behind the nodal point. The visible portion includes therefore only a part of the anterior chamber, a small portion of the lens, and a very small portion of the vitreous. ${ }^{1}$

1 'This matter is clearly' presented by Dr. John Green in his 
2. Repeat Construction 1, but bring the light nearer the observer's eye.

Diminishing the angle between the axis of the observer's eye and the axis of the illuminating pencil increases the length of the cone formed by the intersection of the illuminating pencil and the pencil to the observer's eye. Thus the observer sees a larger cross-section of the lens and vitreous, and sees farther into the eye.

3. Repeat Construction 1, but place the observer's nodal point in the axis of the illuminating pencil.

The point of the cone will reach the retina. The light reflected will emerge from the emmetropic eye in parallel rays which will enter the observer's eye and form upon his retina an image of the illuminated area of the subject's retina.

Influence of Size of Pupil. - Repeat Construction 1 of the preceding section, but enlarge the diameter of the pupil to eight millimetres.

The visible portion of the interior of the eye is greater with a large pupil than with a small one.

Influence of Nearness to Pupil. - Repent Construction 1, but draw the observer's eye nearer the subject's eye.

Note that rays from a larger portion of the article on the Ophthalmoscope printed in the first edition of Wood's lieference Handhook of the Melienl Sciences. 
subject's retina enter the pupil of the observer when the eyes are near.

Ophthalmoscope. - 1. The eye of the observer cannot be placed in the axis of the illuminating pencil without shutting off the illuminating rays. This difficulty was obviated by the invention of the ophthalmoscope.

Place the electric lamp at the same height as the artificial eye, and a little in front of and to one side of it, so that the axis of the illuminating pencil shall be at right angles with the visual axis of the artificial eye. In front of the artificial eye set a clear glass plate at an angle of $45^{\circ}$ to the axis of the illuminating pencil. A portion of the rays which fall upon this plate will pass through the transparent glass and be lost. Another portion will be regularly reflected, and will be thrown into the artificial eye. A portion of the light returning from the interior of the observed eye will be reflected by the glass plate and lost. Another portion will be transmitted through the glass plate in the direction of the visual axis of the observed eye, and may be received by the eye of an observer placed in this axis, as shown in the preceding construction (Helmholtz).

2. Examine the Loring ophthalmoscope. Its essential parts are (1) the mirror of concave 
glass, silvered, pierced at its middle point with an aperture of about $2.5 \mathrm{~mm}$., pivoted to turn to either side; (2) two rotating disks carrying a series of concave and convex lenses in front of the aperture.

The silvered mirror reflects more light than the mirror of transparent glass. Further, it allows the lamp to be placed at the side of the eye to be examined, and at any required distance from the mirror. The turning of the mirror upon a pivot permits the more or less oblique incident rays to be thrown into the eye without tilting the disks carrying the lenses, and thus rendering the lenses astigmatic by placing them at an angle to the optical axis which passes from the subject's retina through the aperture of the mirror and through the lens behind the aperture into the observer's eye.

The disks may be used singly or in combination. A series of concave lenses (marked -) from $1 D$ to $24 D$, and a series of convex lenses (marked + ) from $1 D$ to $23 D$, are thus secured.

\section{Dinect Method}

Emmetropia. - 1. Remove from the lantern the tubes holding the projecting lens. Place the ground glass screen before the condenser. See 
that the inner tube of the artificial eye is drawn out to the line marked zero upon its scale; the eye is then accommodated for distant vision. Set the eye at the level of the observer's eye and near the edge of the table. Place the light on the right side of the artificial eye and slightly behind it. Hold the ophthalmoscope in the right hand close to the right eye at a distance of about fifty centimetres from the artificial eye, and look through the aperture in the mirror. The elbow should be close to the side. The head should be vertical so that the observer's eye and the artificial eye may have the same risual axis. Keep the reflected light upon the pupil of the artificial eye. It will be illuminated by the red reflection from the choroid coat. With the pupil illuminated, approach the artificial eye until the lens-bearing disk lies in the anterior principal focus $(50 \mathrm{~mm}$. in front of this eye, $13 \mathrm{~mm}$. in front of the cornea of the normal human eye; see prge 494.) The artificial eye is accommodated for distant objects. The observer's eye must also be accommodated for distant rision. The power of voluntarily relaxing the ciliary muscle is attained by practice; the observer should endeavor to look through and beyond the eye at some distant object. If the observer be myopic or hypermetropic, his refractive error should be corrected 
by placing the appropriate lens before the opening in the mirror.

As the eye is approached, the details of the fundus will come into view. Find the optic disk. Trace the branches of the central artery and vein which perforate the disk. The image of these parts is virtual, magnified about sixteen times, and erect.

2. Copy Construction 2, page 485 , in which two reduced eyes are placed on the same visual axis facing each other. At the anterior principal focus of the subject's eye draw a concave mirror of $175 \mathrm{~mm}$. focal distance with an aperture of $2.5 \mathrm{~mm}$. through which passes the visual axis.

The rays converging from the mirror and passing throngh the pupil are still further converged, and are brought to a focus in the vitreous, whence they diverge to fall in dispersion circles upon the retina, a large area of which is thus illuminated.

Draw rays reflected from the retina to the pupil of the subject's eye. They must emerge from the eye parallel. Entering the observer's eye, with accommodation relaxed, they will be brought to a focus on the retina. Show by a construction that the image of the optic disk formed in the observer's eye will be inverted but 
will appear to be upright and of its natural size (1.5 mm.).

The apparent size of this image depends upon a visual judgment. The observer knows that small objects are usually held about $250 \mathrm{~mm}$. in front of the nodal point. The size of an object which at this distance would give a retinal image $1.5 \mathrm{~mm}$. in diameter, can be found by formula 5 , page 465 .

$$
B: 1.5:: 250: 15
$$

$B=25 \mathrm{~mm}$., the apparent size of the optic disk viewed by the direct method.

Ametropia; Qualitative Determination. - 1 . Let an assistant make the artificial eye ametropic by moving the draw-tube until the optical axis is shorter or longer than normal. The observer should not know which form of ametropia has been produced. Examine the retina with the ophthalmoscope held from 30 to $50 \mathrm{~cm}$. in front of the artificial eye.

If the details of the fundus can be seen, the eye is either myopic or hypermetropic.

Move the head with the ophthalmoscope from side to side.

If the vessels appear to move in the same direction, the eye is hypermetropic; if in the opposite direction, the eye is myopic.

Measurement of Myopia. - The accommoda- 
tion of the observer's eye and the eye to be examined should be relaxed. The observer's eye must be emmetropic; if it be myopic or hypermetropic, the defect should be corrected by the proper glass before the subject's myopia can be measured. The correction may be made with spectacles, or with one of the lenses in the disk of the ophthalmoscope. The ophthalmoscope should be placed in the anterior focal plane of the eye examined $(13 \mathrm{~mm}$. in front of the cornea of the human eye, $50 \mathrm{~mm}$. in front of the artificial eye). If the observer cannot reach this point, in examining the human eye, the distance between the correcting lens and the anterior principal focus must be subtracted from the focal distance of the correcting lens in order to find the degree of hypermetropia, and be added to the focal distance of the correcting lens in order to find the degree of myopia. Viewed from the anterior principal focus, the fundus will be blurred.

If myopia be present, turn the disk until that concave lens is found which will render clear the image of some one of the vessels ${ }^{1}$ near the border of the optic disk. The rays emerging from the myopic eye are convergent. This lens makes

1 The error introduced by neglecting the distance between the vessels and the nerve elements of the retina is inconsiderable. 
them parallel, and its focal power is the measure of the myopia.

Measurement of Hypermetropia. - If the image of the fundus be blurred by hypermetropia, place convex lenses before the eye until the strongest convex lens is found through which the observer can see clearly the retinal vessel or other point selected. The rays emerging from the hypermetropic eye are divergent. This lens renders them parallel, and its focal power is the measure of the hypermetropia.

Measurement of Astigmatism. - Set the retinal tube of the artificial eye at zero. The eye is now emmetropic. Place before the eye the cylindrical lens of $+2 \mathrm{D}$. Examine the fundus with the ophthalmoscope. The cbserver's accommodation must be relaxed.

The optic disk will no longer appear circular, but will be elongated in the direction of the meridian of greatest curvature. The retinal ressels will not all be in focus. If a horizontal vessel be seen distinctly, and the rertical ressel at right angles to it is blurred, the eye is astigmatic in the horizontal meridian (compare page 423 , and remember that the breadth of the image of the vessel is determined by means of the rays passing through that meridian of the cornea which lies at right angles to the vessel's course.) With the aid 
of the graduated circle on the front of the artificial eye determine the meridian in which the eye is astigmatic. Find the lens which will make the blurred vessel distinct. If the lens, for example, have a focal power of $+2 \mathrm{D}$, there is simple hypermetropic astigmatism of $+2 \mathrm{D}$ in the given meridian. If a lens of $-2 \mathrm{D}$ be required, there is simple myopic astigmatism of $-2 \mathrm{D}$ in the given meridian.

In compound astigmatism, the eye is asymmetrical in more than one meridian. Thus a clear image of the vertical vessels may be obtained with a convex lens of $+2 \mathrm{D}$, while the horizontal ressels may require a lens of $+1 \mathrm{D}$.

The ophthalmoscopic measurement of astigmatism in the human eye is exceedingly difficult, and should always be corrected by more reliable methods.

\section{INDIRECT METHOD}

1. Arrange the light and the artificial eye as directed for the examination by the direct method. Hold the ophthalmoscope $30 \mathrm{~cm}$. from the artificial eye. With the other hand hold a convex lens of $20 \mathrm{D}$ at its own focal length of $50 \mathrm{~mm}$. in front of the cornea. The rays returning from the fundus pass through this lens and form an image in the air between the observer 
and the lens. Examine this image through a magnifying glass of $+5 \mathrm{D}$ placed behind the aperture of the mirror. If the observer be myopic in moderate degree, the aerial image will lie near his far point, and he will need no magnifying or correcting glass; if the myopia be excessive, a weak concave glass should be used. If the observer be hypermetropic, the degree of his hypermetropia should be added to the focal distance of the magnifying glass. The confusing bright reflexes from the surfaces of the $20 \mathrm{D}$ lens may be avoided by holding the lens slightly oblique to the optical axis.

The subject's eye and the $20 \mathrm{D}$ lens form a refracting system like the objective of the compound microscope; the ophthalmoscopic lens plays the part of the ocular.

The image is real, inverted, and magnified. But it will appear to be upright. In it all the relations of the retinal objects are reversed. If the observer move, the image will move in the opposite direction. T'he size of the image is found by formula 5, p. $61 . \quad B$ is the size of the aerial image, $b$ the size of the optic disk $=1.5 \mathrm{~mm} ., g_{1}$ the focal distance of the $20 \mathrm{D}$ lens $=50 \mathrm{~mm}$., $g_{2}$ the distance from the nodal point to the retina $=15 \mathrm{~mm}$. Then

$$
B: 1.5:: 50: 15 \text {, and } B=5 \mathrm{~mm} \text {. }
$$


Thus the enlargement of the retinal details is less than with the direct method. When the aerial image is viewed through the ophthalmoscopic lens of $+5 \mathrm{D}$, an enlarged virtual image of the first image is formed, as in the microscope.

2. Draw constructions showing the formation of the image in the direct and indirect methods. Remember that in the indirect method the rays from the mirror come to a focus before reaching the convex lens. Their second focus is in the vitreous. 


\section{X}

\section{VISION}

Mapping the Blind spot. - Fasten a rod fifteen inches from the table. Beneath the rod place a well-lighted sheet of white paper (a page of the laboratory note-book will serve). Make a small black cross near the left margin. Rest the chin upon the rod in such a way that the right eye shall look directly down at the cross. Place the hand over the other eye. A straw bearing a black pin-head will be drawn by an assistant from the cross along the horizontal meridian toward the temporal side of the eye under observation. The assistant will mark the point where the black object ceases to be visible, and the point at which it reappears. These are the boundaries of the blind spot of the right eye in the horizontal meridian. Determine the boundaries in other meridians. Obtain similarly the outlines of the blind spot of the left eye.

Yellow spot. - Close the eyes for half a minute, and then look at the clear sky or a brightly lighted surface through a solution of chrome alum in a glass bottle with parallel sides. The yellow spot will appear rose-colored 
in the blue-green-red solution. The yellow pigment absorbs some of the blue and green rays. The remaining rays form rose color.

Field of Vision. - Fasten in a vertical position a sheet of white paper about $50 \mathrm{~cm}$. high and $60 \mathrm{~cm}$. broad. (It may be pinned to the wooden stand set on edge upon the electrometer box.) About $20 \mathrm{~cm}$. from the left margin and $30 \mathrm{~cm}$. from the lower margin of the paper mark a small cross. Let the subject rest his chin upon a rod clamped to the iron stand in such a way that the right eye shall look directly at the cross. Cement the squares of black, red, green, and blue papers to the ends of separate straws. Carry the black square from without inwards along the horizontal meridian intersecting the cross. Mark the point at which the black object enters the field of vision. This point is the temporal boundary of the visual field in the horizontal meridian. Determine in the same way the boundary on the nasal side. Repeat for several other meridians. A line joining the points obtained will bound the visual field.

Determine the visual field for red, green, and blue. Always pass the test color from without inwards. The subject should be ignorant of the color to be used, and should name the color as soon as it enters his visual field. 


\section{Color Blindness}

The three large skeins show the test colors.

1. Light Green. - Palest (lightest) shade of very pure green, - neither yellow-green nor blue-green to the normal eye. Light green is chosen because, according to the Young-Helmholtz theory, it is the whitest of the colors of the spectrum, and, consequently, is most easily confused with gray. Light shades are employed because it is difficult to distinguish between strongly illuminated shades.

2. Purple (Rose). - A skein midway between lightest and darkest purple. Chosen because purple combines two fundamental colors which are normally never confounded.

3. Red. - A vivid, slightly yellowish red. Chosen because it represents the color-group in which red (orange) and violet (blue) are combined in nearly equal proportions.

Method of Examination and Diagnosis. - Place the Berlin worsteds on the white cloth in which they are wrapped. They should be well mixed, and not spread out too much. Lay a skein of the first test-color in a well-lighted position two or three feet from the group. Inform the person examined: 
(1) That he must not speak during the test.

(2) That the skeins are not to be fingered or tossed about. A skein should be touched only after its selection.

(3) That he must endeavor to pick out skeins resembling the test skein, i.e., a little lighter or darker in shade; the resemblance cannot be perfect, as no two shades are exactly alike.

Green T'est.--The subject must pick out all the other skeins approximately the same shade.

The color-blind selects some shade of gray.

Purple (Rose). - The subject should pick out the skeins of the same color, as before.

(1) He who is color-blind by the first test, and who, upon the second test, selects only purple skeins, is incompletely purple-blind.

(2) He who, in the second test, selects with purple only blue and violet, or one of them, is completely red-blind.

(3) He who, in the second test, selects with purple only green and gray, or one of them, is completely green-blind.

Remark. - The red-blind never selects the colors taken by the green-blind, and vice versa. Often the green-blind places a violet or blue skein by the side of the green, but only the brightest shades of these colors. This does not influence the diagnosis. 
Red. - This test is applied to those completely color-blind. Continue the test until the person examined has placed beside the specimen all the skeins belonging to this shade, or else, separately, one or more "colors of confusion."

The red-blind chooses (besides the red, green, and brown) shades which to the normal sense seem darker than red. The green-blind selects opposite shades, which seem lighter than red.

Violet Blindness. - Very rare. Recognized by a confusion of purple, red, and orange, in the purple test (see 2). Much care is required to diagnosticate this form.

The Respiration Scheme. ${ }^{1}$ - The glass cylinder (Fig. 67) represents the thorax. The surface of the water in the glass cylinder represents the diaphragm and movable chest walls; its level may be changed by raising or lowering the large rubber tube, in the free end of which is placed a second glass cylinder, not shown in Fig. 67. The interior of the cylinder above the water represents the thoracic cavity, and the rubber balloon the lungs. The paraffined cork is pierced by a pleural and a tracheal tube. The upper end of the pleural tube enters a rubber tube, in the wall of which is a small hole closed by a short glass rod. Through this hole the pleural cavity may be opened to the atmospheric air. The tracheal tube

1 American Journal of Physiology, 1904, x, p. xlii. 
opens below into the lung, above into a rubber tube in the wall of which is a small opening, which represents the glottis, and which may be partly or wholly closed by a glass rod. The left manometer shows the intrathoracic pressure, the right manometer the intra-

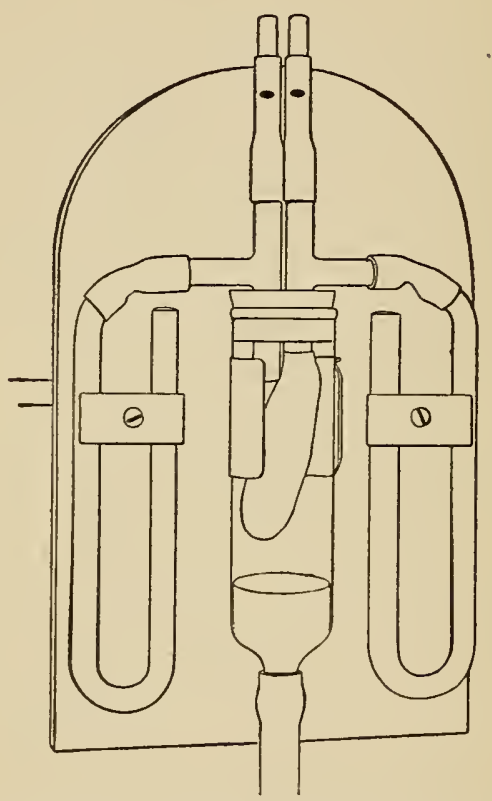

Fig. 67. The respiration scheme; abnut one-third the actual size.

pulmonary pressure. The normal relations between intrathoracic and intrapulmonary respiration may be reproduced with this apparatus. The pressure changes in forced respiration, obstructed air passages, asphyxia, coughing, sneezing, hiccough, and perforation of the pleura may also be studied. 


\section{Xi Mechanics of Riespiration}

Artificial Scheme. - Raise the left glass rod above the opening in the rubber tubing (Fig. 67). Hold the lower end of the free cylinder even with the rubber balloon, and pour in water till the level just reaches the balloon. Lower the left glass rod to cover the opening.

The surface of the water in the attached cylinder represents the diaphragm and movable chest-walls; the interior of the cylinder above the water, the thoracic cavity; and the rubber balloon, the lungs. The left manometer shows the intra-thoracic pressure; the right manometer shows the intra-pulmonary pressure. The left glass rod closes the entrance to the cylinder, i. e. makes the thoracic cavity a closed cavity, as is normal; the right glass rod, with its lower end partly covering the opening in the rubber tubing, controls the entrance to the balloon (the respiratory passages).

Inspiration. - Nearly close the respiratory passage. Lower the water level to the base of the thoracic cylinder.

Note the change in the size of the lung, and in the pressure in the lung and in the thorax. Give reasons for these changes.

Expiration. - Widen the respiratory passage 
slightly. Raise the water level slowly till the lung is slightly but evenly distended.

Note the pressure in the pleural cavity. Is it positive or negative? Why?

Normal Respiration. - Slowly and rhythmically raise and lower the diaphragm (water level) between the inspiratory and expiratory level, taking care that the lung never becomes even slightly collapsed at the end of expiration.

Give reasons for the changes in the intrapulmonary pressure.

Forced Respiration. - Raise and lower the diaphragm more quickly.

Observe that the differences in pressure are increased.

Obstructed Air Passages. - Diminish the inlet in the respiratory tube by moving the glass plug. Raise and lower the diaphragm.

The differences of pressure will be increased.

Asphyxia. - Close the entrance to the lungs entirely.

Note the effect of movements of the diaphragm upon the intra-thoracic and intra-pulmonary pressures.

Coughing: sneezing. - Remove the glass rod from the respiratory passage. Bring the lung to full inspiration. Close the respiratory opening with the moistened thumb. Raise the diaphragm 
half-way toward expiration. Suddenly open the respiratory passage.

Air is quickly and forcibly expelled from the lung (cough, sneeze).

Hiccough. - Lower the diaphragm quickly toward full inspiration, and while the lung is expanding close the respiratory opening with the moistened thumb (hiccough).

Note the sudden changes of pressure in the two cavities.

Perforation of the Pleura. - Open the inlet to the pleura.

Note the effect of the opening into the pleural cavity upon the lung and upon the intra-pulmonary and intra-thoracic pressure.

Observe the result of movements of the diaphragm. 


\section{XII}

THE CIRCULATION OF THE BLOOD

\section{The Mechanics of the Circulation}

The spaces between the cells of which the body is composed are filled with a liquid called the lymph, from which the cells take their food and into which they pour their waste. The materials and the products of metabolism diffuse from lymph to cell and from cell to lymph. In animals in which the division of labor has produced separate organs for digestion, excretion, and the like, the lymph serves as a medium of exchange. For this purpose the relatively slow processes of diffusion are not sufficient. Food must be more rapidly brought and waste more rapidly removed. A circulation must be provided. There are many ways in which the necessary circulation is secured. In Cyclops a flow is caused by movements of the alimentary canal. In Daphnia, the lymph enters a hollow muscle and is then expelled. In the higher animals the provision for rapid exchange is twofold. The intercellular spaces are traversed by a 
countless number of tubes of capillary size, the walls of which are so thin that substances in solution pass through them with great ease. These capillaries are, the ultimate branches of a single tube, and, after fulfilling their function, the capillaries unite into a single tube again. A closed system is thus formed. This system is filled with a modified lymph called the blood, which is kept in coustant circulation. Thus the lymph in the intervascular spaces is in intimate contact with a continually changing liquid. Further provision for rapid exchange is found in the circulation of the lymph itself. The spaces between the cells are drained by channels which gradually become definite tubes, the lymphatics, and these finally join to form two ducts which empty into the blood vessels.

The unbranched portion of the vascular tube is dilated into a cavity with thickened muscular walls termed the ventricle of the heart. The ventricle contracts rhythmically. Each contraction raises the pressure in the ventricle until it is higher than the pressure in the remaining blood vessels. The blood in the ventricle is thereby 'forced into the blood vessels against the resistance of friction. The high pressure in the ventricle during contraction is transmitted into the blood vessels and through them. At each cross- 
section of the vascular system some of the pressure is lost in overcoming resistance; hence the pressure gradually falls. The blood flows from the area of higher pressure, near the ventricle, to the area of lower pressure. Thus the contractions of the ventricle establish a difference of pressure in the blood vessels, which causes a movement of the contained liquid.

At the two points at which the vascular tube joins the ventricle membranous valves are placed. One of these valves opens into the ventricle. It is an inflow valve. The inflow valve closes when the ventricle contracts. Consequently the contractions cannot drive the blood through this orifice. The ventricle can drive the blood only through the remaining orifice. Thus the ventricle becomes a pump and its contractions move the blood always in one direction. The vessels by which the blood is carried from the ventricle to the capillaries are called arteries; those which bring the blood from the capillaries back to the ventricle are called veins. Adjoining the ventricle the great veins meet in a common enlargement called the auricle. It is at the junction of the auricle with the ventricle that the inflow valve is placed.

The outflow valve is placed at that orifice of 
the ventricle which opens into the arteries. When the ventricle, having by its contraction raised the pressure in the arteries, begins to relax, the pressure within its cavity becomes less than that in the arteries. The outllow valve then shuts. Otherwise the arteries would be placed in direct communication with an area of low pressure and the relaxation of the ventricle would undo in part the work of the contraction, the purpose of which was the creation of a pressure in the arteries great enough to force the blood through all the blood vessels.

It is obvious from these general considerations that the problems of the circulation are in the first instance those presented by any system of closed tubes through which liquid is driven by a pump.

The Circulation Scheme. ${ }^{1}$ - The artificial scheme (Fig. 68) to illustrate the mechanics of the circulation in the highest vertebrates cousists of a pump, a system of elastic tubes, and a peripheral resistance. The inlet and the outlet tubes of the pump are furnished with valves that permit a flow in one direction only. The peripheral resistance is the friction which the liquid undergoes in flowing through the minute channels of a piece of bamboo. To this must be added

1 Science, 1905, xxi, pp. 752-754. 
the slighter resistance due to friction in the rubber and glass tubes.

In this system the pump represents the left ventricle; the valves in the inlet and outlet tubes, the

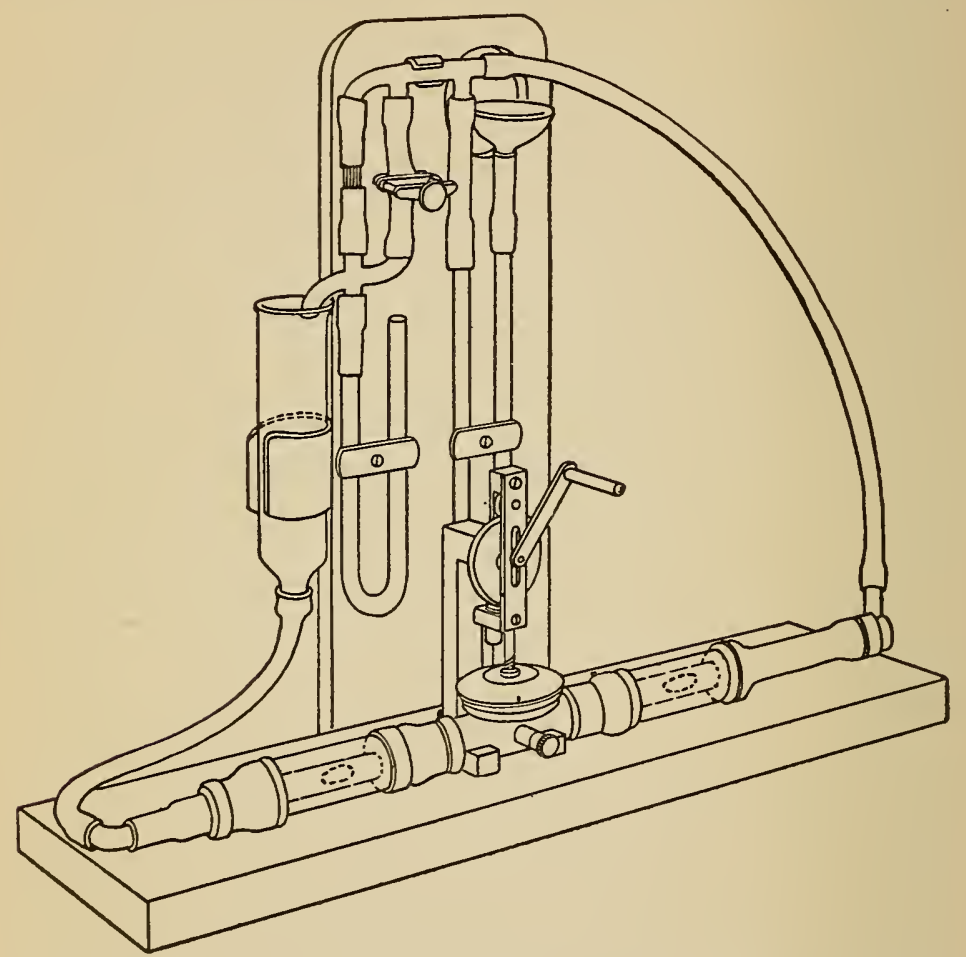

Fig. 6S. Quantitative circulation scheme; about one-fourth the actual size.

mitral and aortic valves, respectively; the resistance of the channels in the bamboo, the resistance of the small arteries and capillaries. The tubes between the pump and the resistance are the arteries; those on the distal side of the resistance are the veins. 
The side branch substitutes a wide channel for the narrow ones, and thus is equivalent to a dilatation of the ressels.

The pressure in the ventricle is varied through a tambour covered with rubber membrane. The membrane is grasped between two disks, one below and one above. The upper disk is screwed down upon the lower until the membrane is tightly held. To these disks is fastened a rod which ends in a yoke. The yoke rests upon a small wheel, which in turn is supported by a brass plate ecentric in form. This brass plate is revolved by turning a handle attached to the axle. As the plate revolves the small wheel bears upon the eccentric rim and rises and falls with the rise and fall in the rim of the plate. The motion of the small wheel is transferred through the yoise, rod, and disk to the rubber membrane, and thus to the interior of the ventricle.

The rim of the eccentric brass plate reproduces the intraventricular pressure curve in the dog. In projecting this curve upon the plate the periphery is divided into fractions of a second, and the radii are divided into millimetres of mercury pressure.

Each revolution of the eccentric plate reproduces in the ventricular tube both the time and the pressure relations of the ventricular cycle in the dog. The intraventricular pressure curve may be written by connecting the side tube with a membrane manometer, and clamping off the arterial mercury manometer to be mentioned shortly. 
When the pressure rises in the ventricle to a sufficient height the contents of the ventricle will be discharged through the aortic valve into the aorta, and thus (through a convenient metal tube) into the arterial tube, leading to the capillary resistance. Here two paths may be taken: the liquid may pass either through the capillary channels in the cane, thus meeting with a high resistance, or this resistance may be lessened to any desired degree by unscrewing a clamp and thus opening the side tube. Both paths lead to the venous tubes, whence the liquid passes through the mitral valve into the ventricle. The mitral and aortic valves are of a modified Williams type. Metal tubes closed at one end conduct the liquid respectively to or from the ventricle. The liquid enters or leaves the valve-tube through a hole covered by a rubber valve-flap, not shown in Fig. 68. Each valve is surrounded by a glass tube through which the working of the valve may be inspected.

Mercury manometers measure the pressure in the arteries and veins near the capillary resistance. The arterial manometer is provided with a glass thistletube to catch any mercury that may be driven out by a careless operator.

If the arterial mercury manometer be replaced by a membrane manometer, or if it be provided with a float and writing point arterial-pressure curves may be written, identical with those obtained from the carotid artery of the dog. 
Normal sphygmographic tracings may be obtained by using a sphygmograph on the aortic tube.

Palpation of the arterial tube will give a pulse the "feel" of which cannot be distinguished from that of the pulse in the normal subject; the pressure waves in the quantitative scheme and in the living animal are identical in respect of both time and pressure.

\section{The Conversion of the Intermittent into A Continuous Flow}

When a pump forces water or any other incompressible fluid through tubes with rigid walls, the inflow and outflow are equal and in the same time. The outflow ceases the instant the inflow ceases. The same is true in a systern of elastic tubes so short and wide that friction between the liquid and the walls causes practically no resistance to the flow. Here the quantity received from the pump can still escape from the distal end of the system during the stroke of the pump. When the resistance is increased by narrowing the tubes, or by increasing their length, or in both these ways, not all the liquid received from the pump can pass by the resistance during the stroke of the pump, - the remainder must pass during the interval between one stroke and the next. The portion which cannot pass during the stroke finds room be- 
tween the pump and the resistance in the dilatation of the containing vessels. To effect the dilatation the force or pressure transmitted from the pump presses out the vessel walls until this pressure is held in equilibrium by the elastic reaction of the walls. As the pressure from the pump wanes, the energy stored by it in the tension of the vessel walls is reconverted into mechanical motion, and the walls return towards their original position, driving the liquid out of the tube past the resistance.

1. Open the side branch by unscrewing the pressure-clip. See that the tubes are well filled with water. Make a single brief gentle pressure on the ventricle.

Note (1) that practically all the liquid driven out by the stroke escapes through the side branch, in which the resistance is low, rather than through the high capillary resistance. (2) Only a portion of the liquid escapes during the stroke. (3) The portion which cannot escape by the resistance during the stroke finds space in a very evident dilatation of the tubes nearer the pump, $i$. e. between the pump and the principal resistance. (4) A membrane manometer coupled to the side tube of the ventricle would show a sudden rise and fall indicating a sudden rise and fall in the intraventricular pressure. (5) Close observation shows that on 
the stroke of the pump the tubing just distal to the aortic valve begins to expand sooner than that farther away. Eviclently the change of pressure produced by the stroke of the pump is transmitted from point to point through the liquid in the tubes. (6). The arterial manometer shows a sudden rise and fall. Observe that the rise is not synchronous with the stroke of the pump, but begins an instant later. This interval is occupied by the transmission of the pressure change from the pump to the mercury column, and in part by the time required to overcome the inertia of position of the mercury. The oscillations of the mercury following the primary rise and fall are due to inertia. (7) Observe the action of the valves (they consist of a metal tube, closed at one end, and pierced with a hole which is covered with a rubber flap tied on both sides of the hole). (8) Place a finger on the "aorta" near the valve and note the pressure wave (pulse) as it passes along the vessel.

2. With the side branch open as in Experiment 1, compress the bulb rhythmically and gradually increase the frequency of stroke.

It will be found that at about twenty strokes to the minute the stream will be intermittent. As the interval between the strokes is shortened the liquid received from the pump in any one 
stroke cannot all escape by the resistance during the stroke and the succeeding interval. The next stroke comes before the outflow from the preceding stroke is finished, and the stream becomes remittent.

Still further increase the frequency of the stroke. A rate will be reached at which onehalf the quantity received from the pump will pass by the resistance during the stroke of the pump and the remaining half will pass in the interval between that stroke and the next; the intermittent will be converted into a continuous flow.

Observe that the duration of the intervals is greater than the duration of the strokes of the pump. Thus the time during which the circulation is carried on by the energy stored by the pump in the elastic walls of the vessel is greater than the time during which it is carried on by the direct stroke of the pump.

Note that the arterial pressure remains low even after the stream becomes continuous. An increase in the frequency of the beat has little influence on the blood pressure where the peripheral resistance is very slight.

3. Close the side branch, so that the liquid must pass through a high peripheral resistance. Compress the bulb at such a rate that the outflow shall be continuous. 
The frequency required to make the flow continuous is now much less than when the peripheral resistance was low.

\section{The Relation between Rate of Flow and Widtì OF BeD}

In a frog slightly paralyzed with curare destroy the brain by pithing, with the least possible loss of blood. Lay the frog back down on the mesentery board. Open the abdomen in the median line. Draw the intestine over the cover glass upon the cork ring so that the mesentery may lie upon the glass evenly and without stretching. The mesentery must be kept constantly moist with normal saline solution. Examine the blood vessels in the mesentery with No. 3 Leitz objective.

Note the swift flow in the larger vessels and the slow movement of the blood through the capillaries.

The combined cross-sections of the capillaries in the body are vastly greater than the cross-section of the arteries or the veins. The total quantity of blood passing in a unit of time through the arteries or veins and the capillaries is the same. If less passed through the capillaries than throngh the arteries, the capillaries would soon be gorged 
to bursting. If more, the arteries would soon be empty. As the quantity passing through the capillaries and the arteries and veins in a unit of time must thus be the same, it follows that where the combined cross-section of the channel or "bed" is small, the blood must flow faster than where the cross-section is large. A river rushes rapidly through a gorge, but moves sluggishly where meadow-lands afford a wider channel. Thus the blood flows with great velocity in the great arteries, less rapidly in their branches, and very slowly indeed in the capillaries, the combined width of which is so great compared to that of the arteries. And as the capillaries unite into the smaller veins, and these into the larger veins, the combined cross-section or bed becomes ever smaller and the blood moves ever more swiftly. Were the slow passage of the blood in the capillaries due simply to friction, the blood would move still more slowly in the veins because the retarding influence of the friction in the veins would be added to that of the capillaries. There is an inverse relation between the rate of flow and the area of bed. 


\section{The Blood-Pressure}

The Relation of Peripheral Resistance to BloodPressure. - Revolve the disk of the artificial. scheme at a rate that will produce a continuous outflow.

With each successive stroke the portion of liquid unable to pass the resistance during the stroke and the succeeding interval is added to that left behind from preceding strokes. The arteries become more and more full. The arterial manometer registers a higher and higher pressure. At length the pressure ceases to rise. The mercury remains at a mean level broken by a slight accession at each stroke. The pump now merely maintains the constant high arterial pressure. This pressure suffices to drive through the resistance during each stroke and the succeeding interral all the liquid received from the pump during the stroke.

The venous pressure remains very low. The capillary resistance (to which must especially be added the resistance of the smallest arteries) almost entirely exhausts the pressure in the arteries. Hence the sudden and profound difference observed between the arterial and the venous pressure. A second arterial manometer placed near the aorta would show that the 
loss of pressure between the ventricle and the smallest arteries is relatively slight.

The pulse is absent on the venous side of the resistance.

The Curve of Arterial Pressure in the Frog. Expose the heart of a frog, the brain of which has been pithed without hæmorrhage. Provide a fine cannula with a short piece of rubber tubing. Fill cannula and tube with one per cent sodic carbonate solution, and close the end of the tube with a small glass rod. Tie a ligature about one aorta as far as possible from the junction of the two aortæ. Knot the ends of the ligature together. Pass a second ligature beneath the same aorta, but do not tie it. Lift the vessel by the second ligature so that the vessel is constricted by lying across the thread. Between the two ligatures open the aorta with sharp scissors and introduce the cannula. Fasten the cannula in place by means of the ligature. Place the frog-board on the wooden stand to bring the heart on a level slightly higher than the level of the mercury in the mercury manometer (Fig. 69). See that the proximal limb of the manometer is filled with one per cent sodic carbonate solution to the exclusion of air. Bring the writing point of the manometer against a smoked drum and revolve the drum once by hand to record a line of atmos- 
pheric pressure. Close the aorta containing the cannula by gentle pressure with a forceps the blades of which are covered with rubber tubing. Join the cannula-tube to the manometer, excluding air bubbles. Remove the forceps.

The mercury will fall in the proximal and rise in the distal limb until the blood-pressure in the aorta is balanced by the column of mercury. With each ventricular beat, the column rises a short distance above the mean level and sinks again.

Record the blood-pressure curve on a very slowly moving drum. To get the actual pressure in millimetres of mercury multiply by two the mean height of the curve above the atmospheric pressure line.

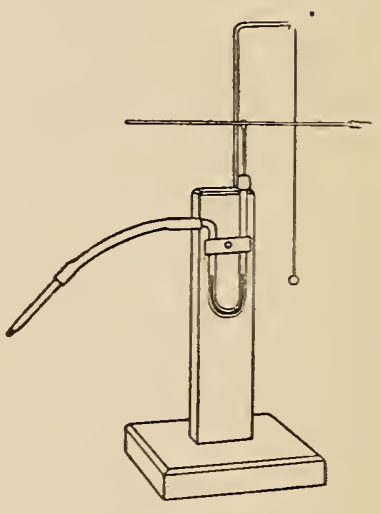

Fig. 69. The small mercury manonieter.

The Effect on Blood-Pressure of Increasing the Peripheral Resistance in the Frog. - The peripheral resistance may be increased by the narrowing of the small arteries which follows the stimulation of special vaso-constrictor nerve fibres. The vaso-constrictor nerves may be stimulated directly or reflexly. The latter method is chosen here. 
Expose the sciatic nerve. Tie a ligature about the nerve near the distal end of the wound, and sever the nerve on the distal side of the ligature. Stimulate the central end with a tetanizing current of moderate strength.

The afferent impulses set up by the stimulation proceed to the spinal cord and thence to the bulb, where they excite nerve cells which discharge impulses that cause the smaller arteries (and probably the veins) to constrict. This narrowing causes the arterial pressure to rise.

Changes in the Stroke of the Pump; Inhibition of the Ventricle. - While the arterial pressure in the artificial scheme is at a good height $(120 \mathrm{~mm}$. $\mathrm{Hg}$ ) arrest the ventricular stroke (the ventricle in animals may be thus inhibited by stimulation of the vagus nerve, page 316 ).

So soon as the ventricle ceases to beat, the less distended arteries will empty themselves through the peripheral resistance, and the arterial manometer will show a continuous fall in bloodpressure.

Resume the ventricular beats.

The mercury in the arterial manometer will rise in large leaps, corresponding to the ease with which the early strokes of the pump distend the lax arteries (the inertia of the mercury somewhat exaggerates the rise at each stroke). As the 
blood-pressure rises, however, the excursion of the mercury for each ventricular stroke becomes less and less, corresponding to the smaller and smaller difference between the pressure in the arteries and the maximum pressure within the ventricle, until at length equilibrium is restored between the peripheral resistance and the force and frequency of the ventricular beat.

The Effect of Inhibition of the Heart on the Blood-Pressure in the Frog. - Arrange an inductorium for strong tetanizing currents. Insert the electromagnetic signal in the primary circuit and bring its writing point beneath that of the manometer. Raise the heart gently. Note the white "crescent" between the sinus venosus and the right auricle. Put the points of the electrodes on the crescent, and close the circuit for a moment. After one or two beats the heart will stop.

Observe the great fall in blood-pressure. Cease the stimulation.

The mercury returns in leaps to its former level.

\section{The Heart as a Pump}

The Opening and Closing of the Valves. - Secure a high arterial pressure $(120 \mathrm{~mm} . \mathrm{Hg})$ in the artificial scheme. Now greatly slow each ven- 
tricular beat and at once observe closely the action of the valves.

It will be seen that the mitral valve closes as soon as the ventricle begins to contract, but the aortic valve does not open until the intraventricular pressure has risen above that in the aorta. Time is required for this rise in the pressure in the ventricle. During this period both mitral and aortic valves are closed. When the ventricle begins to relax, the intraventricular pressure speedily falls below that in the aorta, and the aortic valve shuts, but the intraventricular pressure normally must fall at least $100 \mathrm{~mm} . \mathrm{Hg}$ farther before it shall be lower than that in the auricle. During this fall all the heart valves are again closed; the aortic valves are already shut, and the mitral not yet open.

The Period of Outflow from the Ventricle. - Tie a rubber membrane over the smaller thistle-tube of the sphygmograph (Fig. 70) and cement a bone button in the centre. Connect a membrane manometer ${ }^{1}$ with the side tube of the ventricle. Bring the writing points of the recording tam-

1 If such a manometer is not at hand, carry a thin wire from the yoke of the disk of the circulation scheme to a light muscle lever, counterweighted from the pulley or pulled gently upward by a rubber band attached to the lever. This lever will record the up and down movement of the disk and thus mark the beginning of the ventricular stroke. 
bour and the manometer into the same vertical line against a smoked drum. Let the drum revolve at a fast speed.

Place the button of the receiving tambour on the aorta. It will record the arrtic pulse and the membrane manometer will record the intraventricular pressure. Let the ventricle pump with the usual force and frequency. When the two curves have been writ-

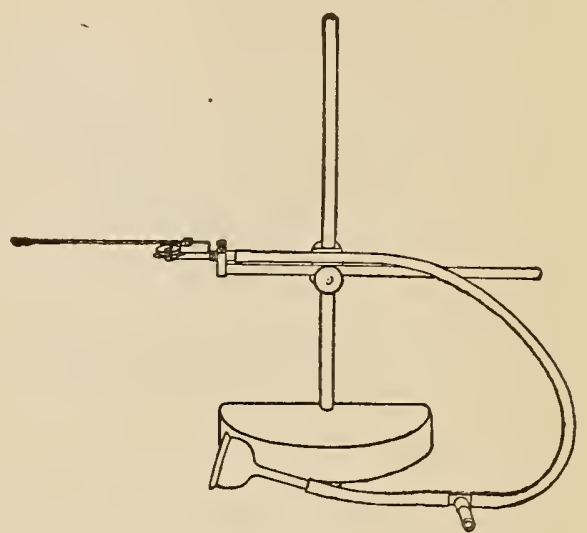

Fig. 70. The sphygmograph. ten stop the clockwork and turn back the drum until the point of the lever recording the ventricular pressure lies at the exact beginning of the upstroke in the aortic pulse curve. Cause each lever to write an ordinate on the stationary drum. These ordinates will indicate synchronous points and will mark the beginning of the "outflow" period.

The Sphygmograph Tambour. ${ }^{1}$ - This small and very sensitive tambour (Fig. 71) is mounted upon a

1 First Catalogue of Harvard Physiological Apparatus, 1901, p. 47. 
hollow tube through which the air waves reach the rubber membrane. A right-angled piece of aluminium transmits the motion of the membrane to the writing lever. The moving parts are of the lightest

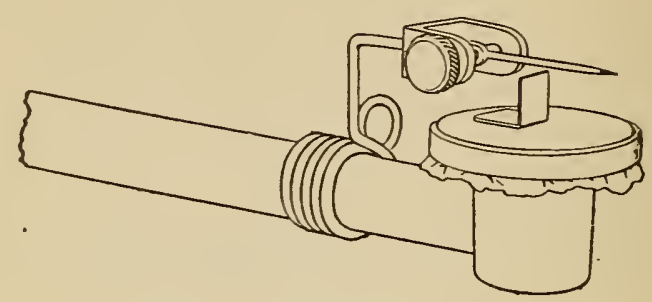

Fig. 71. The sphygmograph tambour; about twice the actual size.

construction. The axle of the writing lever is held in a yoke, the distance of which from the fulcrum of the lever is readily adjustable. The rubber membrane is not tied, but is held in place by a removable ring, a time-saving device.

If a small glass thistle-tube placed over the carotid artery be connected with this tambour by a rubber tube, preferably with a side branch, admirable pulse tracings may be recorded. By covering the thistletube with a rubber membrane upon which a bone button is cementer, sphygmograms may be taken from the radial artery or from the tubes of the circulation scheme. The same tambour is used with the plethysmograph tube. 
Now turn the drum until the point of the aortic lever lies beneath the notch seen in the down stroke of the pulse curve (the dicrotic notch, see page 546). Describe synchronous ordinates. It is known that the dicrotic notch in the aortic pulse curve corresponds closely to the moment of closure of the aortic valves. It marks, therefore, the end of the outflow period. Note that this point is reached soon after the ventricle begins to relax. Thus the period during which the intraventricular pressure is higher than the pressure in the aorta embraces part of the relaxation as well as part of the contraction of the ventricle. It includes approximately the highest third of the intraventricular pressure curve.

Observe also the considerable interval between the beginning of ventricular contraction and the opening of the aortic valve, as shown by the upstroke in the pulse curve consequent upon the entrance of liquid into the aorta.

The Visible Change in Form. - Expose the heart of a frog. Observe the great veins, the auricles, the single ventricle, the two aortæ, and the dilatation, or bulbus, by which the aortie are connected with the rentricle. All these parts except the two aortæ are contracting. The veins contract first; the auricles next; then the ventricle; 
last the bulbus. Note the pallor of the contracted, empty ventricle.

Graphic Record of Ventricular Contraction. Pass a fine wire through the tip of the ventricle and fasten the free end to the heart lever (Fig. 53). Let the lever write on a slow-moving drum.

Note the characteristics of the curve.

\section{The Heart Muscle}

All Contractions Maximal. - Inhibit the heart by a Stannius ligature (see page 562). Find the least strength of stimulus that will cause the ventricle to contract. Increase the strength of the stimulus, but do not stimulate oftener than once in ten seconds (to avoid the staircase contractions described below).

The force of ventricular contraction will remain the same, notwithstanding the increased stimulus.

If the heart responds at all to a stimulus, it responds by a maximum contraction. There is no interval between the minimal and maximal value (compare page 175).

Staircase Contractions. - Find the least stimulus that will cause the ventricle to contract. 
Repeat this minimal stimulus every 5 seconds, recording the contractions on a drum turned about $5 \mathrm{~mm}$. by hand after each contraction.

The contractions of the ventricle will be successively stronger, so that the apices of the curves will form an ascending line ("staircase"). The form of the staircase is always an hyperbola. Successively stronger responses to repeated stimuli of uniform strength can also be obtained from the curarized gastrocnemius of the frog, perfused with blood, and from mammalian and invertebrate muscles. The contraction appears to increase the irritability. Thus the same stimulus causes a greater contraction after a brief tetanus than before. Rossbach and Bohr have observed this after-effect continuing more than thirty minutes.

The Isolated Apex; Bernstein's Experiment.-Draw a ligature about the ventricle halfway between base and apex tightly enough to crush the tissues without wholly separating them. The anatomical continuity between the two halves of the ventricle will thereby be maintained, but the physiological continuity will be lost. Release the ligature.

The isolated "apex" as a rule does not contract. The exceptions can probably be explained 
as the effect of a constant stimulus (see page $533)$.

The apical half of the normal ventricle contains no nerve cells. Consequently its failure to contract after its separation from the remainder of the heart would indicate that the adult heart muscle is incapable of spontaneous rhythmical contraction. It has been shown, however, that the "apex" of the mammalian heart will beat after its complete removal from the remainder of the heart, provided the circulation in the extirpated piece is maintained by supplying it with blood.

Rhythmic Contractility of Heart Muscle.- Further evidence of the rhythmic contractility of the heart muscle is found in the bulbus arteriosus.

Place very small pieces of the bulbus arteriosus in normal saline solution under the microscope.

They will contract rhythmically.

Histological examination shows that nerve cells seldom occur in the bulbus. It is scarcely credible that they are present in each of the small pieces seen contracting under the microscope.

Constant Stimulus may cause Periodic Contraction. - In a frog with ventricular apex isolated by Bernstein's ligature, compress one or both aortix, thus raising the pressure in the ventricle. 
The increased intracardiac pressure acts as a constant stimulus to the cardiac muscle and the hitherto inactive apex begins to contract again.

Thus a constant stimulus may discharge periodic contractions in a muscle habituated to periodic contractions (compare page 144); the galvanic current and chemical stimuli, such as delphinin, are further examples of constant stimuli which call forth rhythmic contractions of the heart muscle.

The Inactive Heart Muscle still Irritable. - Stimulate the inactive "apex" mechanically and with single induction shocks.

The apex, though incapable of spontaneous rhythmic contractions, is still irritable, and will respond by a single contraction to each stimulus.

Refractory Period; Extra-Contraction; Compensatory Pause. - Put the electromagnetic signal in the primary circuit. Connect the binding posts on the heart-holder to the secondary coil of the inductorium. Arrange the latter for single induction currents. Place the rentricle on the heart-holder. Send maximal make and break induction currents through the ventricle from time to time in each phase of the cardiac cycle.

Note that (1) the stimulus sometimes calls forth an extra-contraction; (2) at other times the stimulus causes no contraction, having fallen 
into the ventricle during the period in which it is refractory towards stimuli; (3) the extra-contraction is followed by a pause, called the compensatory pause because it usually restores the rate of beat to that existing before the extracontraction took place.

Using induction currents of equal intensity, find the limits of the refractory period and note them on the drum. Note also the point in the cardiac cycle at which the maximum extracontraction can be obtained.

The Transmission of the Contraction Wave in the Ventricle; Engelmann's Incisions. - The action current of the heart is taken to be an expression of the excitation process, although the nature of the latter is not yet understood. It has already been shown (page 310) that the action current sweeps rapidly over the ventricle preceding the contraction. The excitation might be propagated by nerves or by muscle fibres. The following experiment affords some evidence that the transmission is by means of muscular tissue.

Leaving the heart in situ, cut the ventricle into a zigzag strip by obliquely transverse incisions beginning near the apex. The nerve fibres in the ventricle will thereby be severed at some part or other of their course, but muscular continuity will be preserved. 
The contraction wave will pass over the entire zigzag strip. Normally the wave starts at the base and proceeds to the apex, but by artificial stimulation it can be made to pass from the apex towards the base. A similar result can be secured with the auricle.

The Transmission of the Cardiac Excitation from Auricle to Ventricle; Gaskell's Block. - The contraction wave can be seen to begin normally in the sinus and thence to pass rapidly over the auricle; on reaching the auriculo-ventricular junction there is a distinct pause termed the auriculo-ventricular interval; finally, the excitation reaches the ventricle, and the contraction wave is seen to traverse the ventricular muscle as noted above. The auriculo-ventricular interval may be lengthened by any natural or artificial hindrance to the passage of the excitation wave.

1. Place the Gaskell clamp about the auriculoventricular junction. Very cautiously turn the screw until the rubber edge makes a gentle pressure on the cardiac tissues at that point.

With careful work a degree of pressure will be reached that diminishes the conductivity of the muscle fibres joining the auricle and ventricle so far as to permit only every second or every third excitation to pass. The auricle will beat with- 
out change of frequency, but the ventricle will contract only when the excitation succeeds in passing the block.

2. Divide the auricles in two pieces connected by a small bridge of auricular tissue. Stimulate one piece.

The stimulation of one piece will be followed immediately by the contraction of that piece, and, after an interval, by the contraction of the other. The smaller the bridge, the longer the interval.

Gaskell has pointed out that a natural block is furnished by the small number of the muscle fibres joining the auricle to the ventricle, and that this natural block explains the auriculoventricular interval, $i$. $\iota$. the delay which the excitation experiences in passing from the auricle to the ventricle.

3. Repeat Experiment 1, but place the screwclamp across the middle of the ventricle.

The passage of the excitation from one part of the ventricle to another will be delayed or interrupted by the lowering of the conductivity in the compressed portion.

Many irregularities in the frequency and force of the heart can be explained by variation in the conductivity of its several parts. They can be 
explained also by variations in the irritability of the several parts. In the latter case, the excitation would pass as usual, but its action on any part, for example the ventricle, would be increased or diminished by changes in the irritability of the cardiac muscle in that region. Engelmann has found that ventricular systole lowers the conductivity of the ventricle for a time.

Tonus. - Pass the very fine copper wire through the wall of the auricle of the tortoise and attach the wire to the heart lever, so that the contractions of the auricle may be recorded. Let the drum move so slowly that the individual contractions will be nearly but not quite fused.

Two sorts of contractions can be distinguished, (1) the usual frequent contraction or beat of the auricle, (2) the tonus oscillations. The tonus oscillations include from twenty to forty beats. In the tortoise auricle, the beats usually become less extensive during the rise of tonus.

The Influence of "Load" on Ventricular Contraction. - Record the contractions of the frog's ventricle. Increase the intraventricular pressure (i.e. the load against which the ventricular muscle contracts) by clamping the aortre with forceps 
the blades of which are covered with rubber tubing.

The force of the individual contractions will be increased but their frequency will be diminished.

The Influence of Temperature on Frequency of Contraction. - Let the drum move at such a speed that the individual heart-beats in the curve shall be close together, but yet separate and distinct. Surround with normal saline solution at $25^{\circ} \mathrm{C}$.

The frequency of contraction will be increased.

Replace the warm solution with normal saline solution at $5^{\circ} \mathrm{C}$.

The frequency of contraction will be diminished.

The Action of Inorganic Salts on Heart Muscle.Sever the apical two-thirds of the ventricle of the tortoise heart from the remainder of the ventricle by a cut parallel with the auriculo-ventricular. furrow. With a second parallel cut remove from the severed portion a ring two or three millimetres wide. Divide the ring to form a strip. Fasten one end of the strip to the short limb of a glass rod bent at a right angle. By means of a silk thread connect the other end of the strip to a heart lever arranged to record the 
contractions of the strip on a very slowly moving drum.

Sodium. - Immerse the strip of ventricular muscle in a beaker containing 0.7 per cent solution of sodium chloride.

After a latent period, which may be protracted, but usually is brief, a series of rhythmic contractions will be observed. The contractions soon reach a maximum and then gradually die away. Sodium, although an important stimulus to contraction, cannot maintain the ventricle in continued activity.

The tonus of the heart muscle is diminished by sodium chloride.

Calcium. - Surround a strip of contracting ventricular muscle with a solution of calcium chloride isotonic with 0.7 per cent-sodium chloride solution (approximately 1.0 per cent).

Contractions will cease. Calcium added to solutions of sodium chloride, however, will lengthen the period during which the heart muscle contracts and will increase the strength of the individual contractions. Strong solutions of calcium chloride greatly increase the tonus.

Potassinm. - Surround a non-beating strip of ventricular muscle with a solution of potassium chloride isotonic with 0.7 per cent sodium chloride solution (approximately 0.9 per cent). 
Contractions will not be produced. If potassium be applied to a contracting strip, the contractions will cease.

Combined Action of Sodium, Calcium, and Potassium. - Surround the ventricular muscle with a solution containing sodium chloride $(0.7$ per cent), calcium chloride (0.0026 per cent), and potassium chloride ( 0.035 per cent). This is a modified "Ringer" solution.

Long-continued, rhythmic contractions will be secured.

Observers are not entirely agreed as to the action of potassium and calcium on heart muscle. The matter is of importance because there is much probability that the rhythmic contractions of the heart are the result of the constant chemical stimulus of inorganic salts present in the blood. Most observers are agreed that the interaction of salts of sodium, calcium, and potassium is essential.

The fact that the contraction of the heart begins normally in the sinus may be due to a greater sensitiveness of that part to chemical stimulation. 


\section{The Heart Sounds}

With a binaural stethoscope auscultate the chest over its entire extent during normal respiration and while the subject holds his breath.

1. Note that two sounds are heard in the heart region.

2. Determine at what point each of the sounds is most distinct.

It will be found that one, termed the "first sound," will be most distinct where the ventricle comes nearest the surface, near the apex of the heart, in the space between the fifth and sixth ribs, about $2.5 \mathrm{~cm}$. below and $2.5 \mathrm{~cm}$. within the left nipple. Close inspection of this region in persons not too fat will show that the chest wall is raised at each contraction of the heart. The cardiac impulse, as it is called, may be felt distinctly by one or two fingers laid in the fifth intercostal space. It is caused by the rapid increase in the tension of the ventricle.

The "second sound" will be heard most distinctly immediately over the aortic arch, near the junction of the second right costal cartilage with the sternum.

3. Observe the two sounds with relation to their duration, pitch, intensity, and quality. 
The first sound in comparison with the second is of longer duration, lower pitch, and greater intensity. The quality of the first sound is dull, booming; that of the second is sharp, valvular.

4. With one finger feeling the cardiac impulse observe the sounds with reference to systole and diastole.

The first sound will be found to be systolic, $i$. e. it occurs with the contraction of the ventricle, while the second sound is diastolic, being heard at the beginning of ventricular relaxation. The interval between the first and second sounds is therefore very brief. The pause after the second sound before the first is heard again, is considerably longer.

The first sound can be heard in the extirpated, bloodless heart (dog). The contraction of the ventricular muscle is therefore alone sufficient for its production. But the sound is modified or replaced by a murmur when the auriculo-ventricular valves are sufficiently injured. It is probable, therefore, that the sudden increase in the tension of the auriculo-ventricular valves contributes to its production. The second sound obviously is due to the sudden increase in the tension of the semilunar valves. It is replaced by a murmur when these valves are rendered incompetent. 
Ordinarily the ratio between the blood-pressure in the pulmonary artery and right ventricle so nearly equals the ratio between the blood pressure in the aorta and left ventricle that the semilunar valves in the pulmonary artery and aorta close together, or nearly together, and their respective sounds are heard as one. Pathologically, for example in distention of the right heart from prolonged violent exercise, these relations may be so altered as to produce between the two sounds an interval perceptible to the ear. The sound is then said to be reduplicated.

\section{The Pressure-Pulse}

Frequency. - Palpate the radial pulse by laying on the artery at the wrist the ball (not the tip) of the first, second, and third fingers of the right hand. The forearm of both subject and observer should be supported in a comfortable position. Count the pulse in four successive periods of fifteen seconds. The counting of the observer's instead of the subject's pulse may be avoided by noting whether the subject's supposed pulse is synchronous with the observer's heartbeat.

Note the frequency per minute when the subject is standing, sitting, lying, swallowing, holding the breath; and before and after exercise; 
for example, before and after lifting the weight of the body ten times by rising on the toes.

Sex, eating, the time of day, the temperature, and many other factors also influence the frequency of the pulse.

Hardness. - When pressure is made upon an artery in any part of its course, the pressure is transmitted in all directions through the liquid contained in the peri-arterial tissues, and the artery becomes smaller. Part of the pressure is used upon the peri-arterial tissues themselves. When the remaining pressure equals the maximum blood-pressure in the artery at the point of compression, the blood-pressure on the distal side of this point will sink to the level of the blood-pressure in the nearest anastomosis. If the anastomosis is of capillary size, the pulse will disappear. A pulse which is obliterated by slight pressure is termed "soft;" if the pressure required is relatively considerable, the pulse is termed "hard." The hardness of the pulse is therefore a measure of the maximum bloodpressure at the point of compression, less the variable and unknown quantity required for the compression of the elastic tissues.

Form. - 1. The vibrations which follow the primary pulse wave cannot ordinarily be recognized by the palpating finger. When, however, 
the usual amplitule of the principal secondary vibration is much increased and the interval between the primary and this secondary vibration is not too brief, the pulse may be felt to be double, or "dicrotic." For example, dicrotism can be felt in some cases of continued fever.

2. A pulse which is felt to reach its maximum slowly is called a "slow pulse" (pulsus tardus). One which reaches its maximum rapidly, giving the palpating finger the sensation of a quick push, is said to be a "quick pulse" (pulsus celer). Quick and slow pulses should be carefully distinguished from frequent and infrequent pulses.

Volume. - The extent to which the arterial wall is driven from its position of equilibrium (volume or size of pulse) is a function of the output of the ventricle, the outflow period, the peripheral resistance, and the elasticity of the arteries. It is measured very inexactly by the palpating finger and the sphygmograph, accurately by the plethysmograph (page 552).

The Pressure-Pulse in the Artificial Scheme. Revolve the disk of the artificial scheme until the arterial pressure is maintained at $50 \mathrm{~mm}$. Hg. Close the tube leading to the arterial manometer, so that the oscillations of the mercury may not influence the curves to be taken. Attach the small thistle-tube (without 
rubber membrane) to the sphygmograph (Fig. 70 ) and adjust the tube upon the aorta. Close the side branch of the sphygmograph tube. Bring the writing point of the sphygmograph lever against a slow-moving, lightly-smoked drum. Record a series of pulse curves.

Note the quick upstroke, corresponding to the quick distention of the artery by the emptying of the ventricle, and the gradual downstroke, corresponding to the gradual emptying of the artery through the resistance during the diastole or interval between two beats. Near the apex of the more delicately written curves may be seen a slight depression, the dicrotic notch.

It is obvious that the changes observed in the size of the artery are the expression of changes in the blood-pressure. The pulse is a function of the blood-pressure at the point observed. Hence the term pressure-pulse.

The Human Pressure-Pulse Curve. - 1. Adjust the lever of the recording tambour so that it shall write with the least friction possible on a thinly smoked drum. Let the drum revolve slowly (two revolutions a minute). Be sure that the side branch is open. Place the larger thistletube, which serves as a "receiving tambour," over the carotid artery, anterior to the sternocleidomastoideus muscle, about the level of the 
thyroid cartilage. When the tambour (without rubber membrane) is pressed well down over the artery, let an assistant close the side branch. If the receiving tambour has been properly placed, the recording tambour will write a sharply marked pulse curve. If none such appears, open the side branch and move the receiving tambour into a better position.

Indicate the primary wave, the predicrotic elevation, and the dicrotic notch.

2. Cover the thistle-tube with a rubber membrane. Cement in the centre of the membrane a bone collar-button. Place the button upon the radial artery at the wrist and record the radial pulse.

It will be found that the degree of pressure must be carefully regulated in order to secure a satisfactory curve. The blood-pressure in the artery normally is held in equilibrium by the elastic tension of the wall of the artery and the surrounding tissues. The pressure of the sphygmograph increases the tension of the peri-arterial tissues and thus assists in holding the bloodpressure in equilibrium. The greater the pressure of the sphygmograph, the larger the part of the blood-pressure borne by it and the more completely will variations in the blood-pressure be made visible in the pulse curve. The record, 
however, is not a measure of the absolute bloodpressure, because it is not possible 'o estimate accurately how much of the blood-pressure is still held in equilibrium by the elastic tension of the arterial wall and the surrounding tissnes. The pulse curve does give with approximate correctness the variations in the blood-pressure. The correctness would be complete were it not that the part of the blood-pressure held in equilibrium by the elastic tension of the arterial wall varies with the size of the vessel, and the size of the vessel increases as the blood-pressure increases. Thus the portion of the blood-pressure which fails of record constantly varies. The error thus introduced is not important. The sphygmograph, therefore, gives a practically true record of the form of the pulse, i.e. the time-relations of the changes in blood-pressure. This knowledge cannot possibly be secured by the palpation of the pulse. The sphygmograph, it may be repeated, does not give a true record of the absolute blood-pressure (hardness) or of the amplitude (size) of the pulse. Both hardness and amplitude are better measured by the palpating finger.

In many sphygmographs, for example, Marey's and Dudgeon's, the pressure on the artery is made by a metal spring, the movements of which 
are recorded by a lever. In the record just taken from the radial artery, the pressure was made by the elastic tension of the rubber membrane closing the thistle-tube. In the case of the carotid artery, this membrane is replaced by the skin of the neck.

In every instance, the sphygmograph records the changes of blood-pressure in a section of the artery so short in comparison with the length of the whole arterial tree as to be practically a cross-section.

Low Tension Pressure-Pulse. - 1. In the artificial scheme open slightly the side-branch that permits the liquid in the arterial tubes to flow out without passing through the resistance. The arterial pressure will fall in consequence of the diminished peripheral resistance. Normally this effect is produced by a dilatation of the smaller arteries. Let the arterial pressure fall to about $20 \mathrm{~mm}$. Hg. Record a series of pulse curves.

Note that the oscillations of the mercury column with each ventricular beat are much higher than with normal pressure (120-150 mm.). Feel the pulse with the finger. With each beat the artery quickly expands and as quickly relaxes. The artery is "softer" than usual.

2. Feel the normal pulse in the radial artery. Note the normal " hardness." Let the subject 
inhale two drops (on no account more than two) of the nitrite of amyl (to be dropped on a handkerchief by one of the instructors). This powerful drug causes dilatation of the blood vessels, particularly the smaller arteries.

Observe that as the face flushes, indicating the vascular dilatation, the pulse will be softer.

Do not repeat the experiment.

Pressure-Pulse in Aortic Regurgitation. - Empty the principal tubes of the artificial scheme. Remove the rubber from about the aortic valve. Replace the valve tube. Fill the apparatus with water. Revolve the disk at the rate and with the force employed to imitate the normal circulation (page 545).

Feel the pulse with the finger.

After each systole the liquid streams back through the incompetent valve. The ventricle is thus fuller than normal at the beginning of the stroke, while the arteries are less than normally full. Consequently more than the usual quantity is discharged by the ventricle into relatively undistended arteries. The relatively lax artery is thereby quickly and largely expanded, as indicated by the quick thrust given the palpating finger and by the large excursion of the mercury in the arterial manometer.

Record pulse curves. 
The upstroke is unusually high and quick. It is at once followed by a great and sudden fall. Obviously a relatively empty artery has been suddenly filled by an unusually large inflow and has been suddenly emptied again through the broken valve and the capillaries. The pulsecurve shows low arterial tension, but is of greater amplitude than the pulse in which low tension results from lowering the peripheral resistance. In the body, the amplitude of the pulse in aortic regurgitation is increased by the greater force with which the ventricle contracts, as well as by the larger quantity discharged at each beat, for the back-flow from the aorta dilates the ventricle and usually causes the walls of the ventricle to increase in thickness (dilatation with hypertrophy of the ventricle).

Stenosis of the Aortic Valve. - Replace the rubber flap upon the aortic valve-tube, and tie a string around the flap and tube just over the opening in the tube. Stenosis, i.e. narrowing, of the opening will thus be secured. Put the valvetube in place, and compress the bulb at the usual rate. Record pulse curves.

The slow difficult emptying of the ventricle will be evident in the curve and to the hand. The movements of the arterial manometer are sluggish and of diminished amplitude. The 
pulse wave is small and the upstroke slow, corresponding to the small slow inflow through the stenosed valve.

Restore the valve to its normal state.

Incompetence of the Mitral Valve. - Remove the rubber flap from the mitral valve. Record pulse curves as before.

The pulse will be small, because the pressure in the auricle (in this case the reservoir of water) is always low, while the pressure in the arteries is always high. Hence the ventricle will partly empty itself through the incompetent mitral valve, in the direction of low resistance, before the pressure in the ventricle rises high enough to open the aortic valve against the high aortic pressure. The quantity remaining in the ventricle when the intraventricular pressure rises high enough to open the aortic valve is not sufficient to distend the arteries to the normal degree.

In mitral stenosis the pulse is also small because the narrowing of the mitral orifice permits less than the usual quantity of liquid to enter the ventricle.

\section{Tine Volume Pulse}

Renove the receiving tambour of the sphygmograph from its tube, and insert the plethysmograph cylinder (this is the tube used in the 
experiment on the volume of contracting muscle, Fig. 58). Place the middle finger in the cylinder, making sure that the rubber collar fits around the finger tightly, but without impeding the venous circulation. Close the side branch.

Periodical alterations in the volume of the finger will be recorded; they have the rhythm of the heart-beat. (The friction of the writing-lever must be very slight to insure success, and the curve at best will be small.)

Determine the effect of straining and forced respiration upon the curve.

\section{Apparatus}

Normal saline. Bowl. Towel. Pipette. Artificial scheme. Microscope. Mesentery board. Mercury manometer. Aortic cannula. One per cent solution of sodic carbonate. Ligature. Glass rod one inch long. Frogboard. Wooden stand. Kymograph. Inductorium. Dry cell. Electrodes. Key. Electromagnetic signal. Sphygmograph with large anu small thistle-tubes. Rubber membrane. Bone collar-button. IIeart-holder. Screwclamp. Muscle lever with scale-pan and weights. Stand. Fine copper wire. Tortoise with heart exposed. Ice. Solution of sodium chloride, 0.7 per cent. Solutions of calcium chloride, and potassium chloride, each isotonic with 0.7 per cent solution of sodium chloride. A solution containing sodium chloride, 0.7 per cent; calcium chloride, 0.026 per cent; and potassium chloride, 0.035 per cent. Binaural stethoscope. Nitrite of amyl. Plethysmograph. 


\section{XIII}

\section{THE INNERVATION OF THE HEART AND} BLOOD-VESSELS

THE quantity of blood required by the tissues varies from time to time. For example, the digestive organs require more blood when food is taken than at other times. Variations in the blood supply of the individual organs are accomplished chiefly by varying the size of their blood vessels. To this end the blood vessels are provided with muscular coats which are made to contract or relax, and thus to constrict or dilate the vessels. The impulse to contraction or relaxation is given by the vasomotor nerves. It is necessary, too, that the force and frequency of ventricular contraction should vary with the resistance to be overcome, the need for more rapid oxygenation of the blood, etc., and special nerves are provided for this purpose also. The control or innervation of the heart and blood vessels will now be considered.

The heart is provided with nerves that augment and nerves that inhibit its action. 


\section{The Augmentor Nerves of the Heart}

In the frog both the augmentor and the inhibitory nerves reach the heart through the splanchnic branch of the vagus. The augmentor fibres leave the spinal cord in the third spinal nerve, and pass through the ramus communicans of this nerve into the third sympathetic ganglion, where they probably end in contact with the body or processes of sympathetic cells. The axis-cylinders of these sympathetic cells pass up the cervical sympathetic chain to the ganglion of the vagus (Fig. 72), and thence down the vagus trunk to the heart. Thus in the greater part of its course the vagus cannot be stimulated without exciting both the augmentor and the inhibitory cardiac fibres. To excite either alone it is necessary to stimulate the respective nerves above their junction.

Preparation of the Sympathetic. - Cut away the lower jaw of a large frog, the brain of which has been destroyed by pithing, and continue the slit from the angle of the mouth downwarks for a short distance. Avoid cutting the vagus nerve (Fig. 73). Turn the parts well aside, and expose the vertebral column where it joins the skull. Remove the mucous membrane corering the roof of the mouth. The sympathetic is situated 
immediately under the levator anguli scapulæ muscle, which must be carefully removed. The nerve will then be visible. It is commonly pigmented and usually lies under an artery. Carefully isolate the nerve. Put a ligature around it

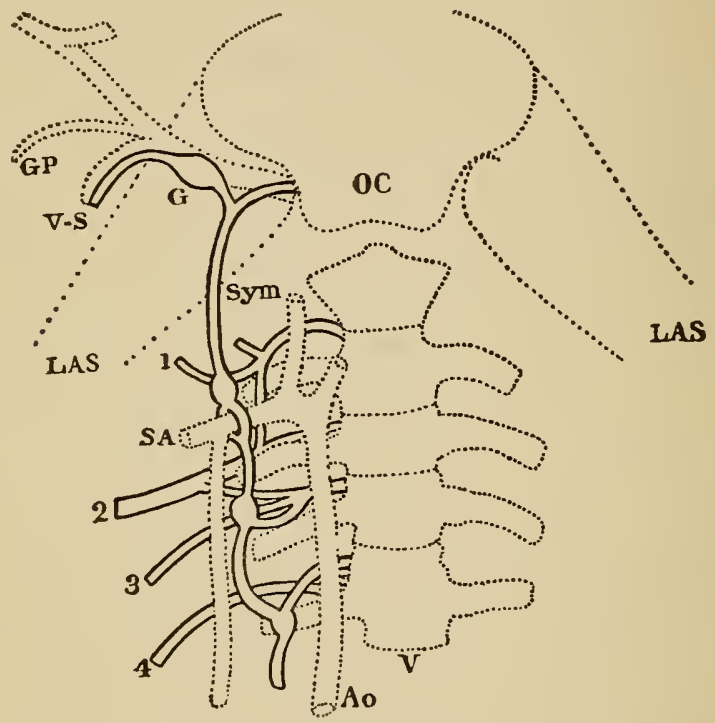

Fig. 72. Seheme of the sympathetic nerve in the frog. OC. Occiput. LAS. Levator anguli scapulæ. Sym. Sympathetic. GP. Glosso-pharyngeus. V-S. Vago-sympathetic. G. Ganglion of the vagus. Ao. Anrta. SA. Subelavian artery. (After Stirling's reproduction of Gaskell and Garlow's plate.)

as far away from the skull as practicalle, and cut the nerve caudal to the ligature.

Action of the Sympathetic on the Heart. Arrange the inductorium for weak tetanizing currents. In the primary circuit place the electro- 
magnetic signal. Prepare the sympathetic as directed above. Expose the heart (page 75). Place it in the heart-holder. Should the heart beat rapidly, slow it with ice. Let the writing point record above the point of the electromagnetic signal on a drum revolving so slowly that the individual beats shall appear in the curve very close together, yet far enough apart to be readily counted. Divide the observation into nine periods of twenty seconds each. Place the electrodes beneath the sympathetic, with the short-circuiting key closed. Adjust the heart lever to write its curve. Let the assistant call the beginning of each period as he marks it on the drum. At the beginning of the second period, open the short-circuiting key; at the beginning of the third period, close the short-circuiting key. Lower the drum when one circuit is completed.

Count the number of beats in each period. The frequency will be increased. The force of contraction will also be increased. ${ }^{1}$ The latent period of excitation is long and there is a prolonged after-effect. The former frequency is regained more rapidly after short than after long stimulations. The speed of the cardiac excitation wave

1 The stimulation of the augmentor fibres is difficult and often fails in winter frogss. 
(compare page 336) is increased and the time of its passage across the auriculo-ventricular groove is shortened, though this cannot be observed by the method used in the present experiment.

\section{The Inhibitory Nerves of the Heart}

The Preparation of the Vagus Nerve. - Fasten a large frog on the board, back down. Pass the

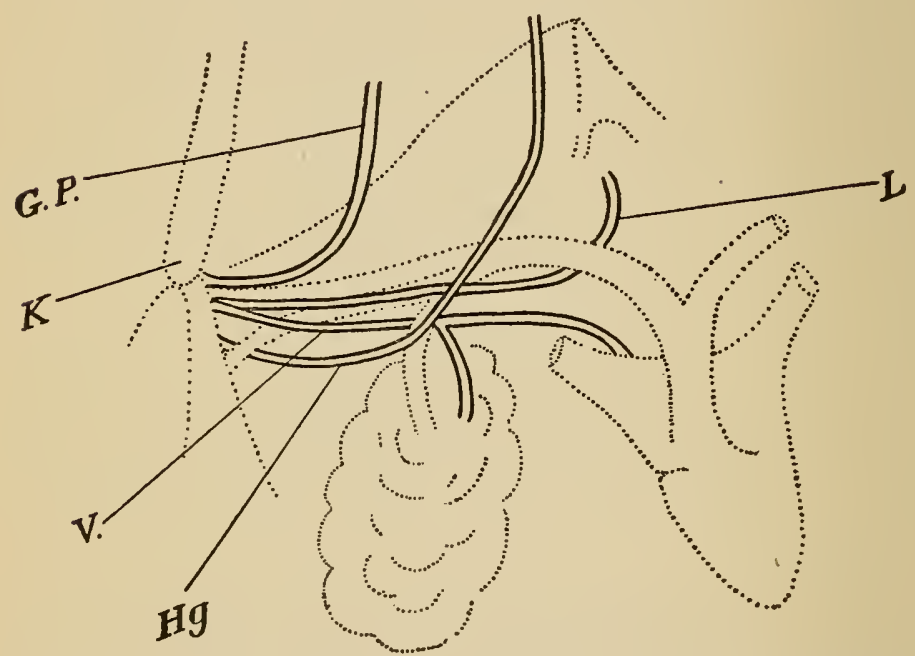

Fig. 73. Scheme of the cervical nerves in the frog (after Schenck). G. P. Glosso-pharyngeus. Hg. Hypoglossus. V. Vagus. L. Laryngeus. K. Posterior end of lower jaw. The glosso-pharyngeus bas been drawn to one side of the hypoglossus for the sake of clearness.

glass tube through the œosophagus into the stomach. Remove the muscles lying over the petrohyoid muscle, which passes from the base of the skull to the horn of the hyoid bone. Lying 
near the line between the angle of the jaw and the auricle are four nerves (Fig. 7:3): (1) The hypoglossus. This nerve is superficial. Near their energence from the skull it is the lowest of the nerres, but later, the uppermost. It crosses the remaining nerves and the blood-vessels, and passes forwards and inwards towards the tongue. (2) The glosso-pharyngeus, which soon turns forwards beneath the hypoglossus parallel to the ramus of the jaw. (3) The vagus, and (4) the laryngeus, the two lying almost parallel in the line between the angle of the jaw and the auricle. The laryngeus rests on the petrohyoid muscle, and passes upwards and inwards beneath the arteries towards the larynx. The vagus runs at first along the superior vena cava to the auricle; a branch is given off to the lungs. Clear the vagus, tie a silk thread around the nerve and sever the nerve on the cranial side of the ligature, so that the peripheral stump can be placed on the electrodes for stimulation. Divide the laryngeal branch. Keep the preparation moist with normal saline solution.

Stimulation of Cardiac Inhibitory Fibres in Vagus Trunk. - Arrange the inductorium for weak tetanizing currents. In the primary circuit place the electro-magnetic signal. Expose the heart. Place it in the heart-holder. Let the 
writing point record exactly above the point of the electromagnetic signal on a drum revolving so slowly that the individual beats shall appear in the curve very close together and yet far enough apart to be readily counted.

Lay the vagus nerve on the electrocles. Start the drum. As soon as good curves are writing, start the inductorium, and open the short-circuiting key for about twenty seconds. The heart will be inhibited. Note that the arrested heart is always relaxed, $i . e$. in diastole. The latent period is short (one or two heart-beats). A brief aftereffect is present. If the stimulus is continued, the heart will begin to beat even during the stimulation, showing that the inhibitory mechanism can be exhausted. The heart beats more rapidly, and usually more strongly, immediately after inhibition than before; this probably is due to the after-effect of the stimulation of augmentor fibres in the vagus trunk, as explained below.

Repeat the stimulation, but weaken the stimulating current by moving the secondary farther from the primary coil.

With a suitable strength of current, the heart will be slowed but not arrested. The duration of diastole will be markedly less, while the duration of systole will be changed but little if at all. A stronger excitation would lengthen both 
systole and diastole. The diminution in force often appears before the diminution in frequency.

Effect of Vagus Stimulation on the Auriculo-Ventricular Contraction Interval. - Counterpoise two inverted muscle levers. Place their writing points exactly above the writing point of the electromagnetic signal. Pass fine bent pins through the auricle and ventricle, respectively, and connect them by silk threads with the muscle levers ("Suspension method"). Let the drum revolve at its fastest speed. When good auricular and ventricular contractions are obtained, stimulate the vagus trunk with a current not quite sufficient to cause arrest.

Note that the inhibition affects both the auricle and the ventricle. Weak stimuli affect primarily the auricles. The auriculo-ventricular contraction interval is lengthened.

Irritability of the Inhibited Heart. - Arrest the heart by stimulating the vagus trunk. When complete inhibition is secured, touch the ventricle smartly with the point of the seeker.

The ventricle will respond by a single contraction.

When the inhibition is profound, the irritability may be so far reduced that the heart will not contract on direct stimulation.

In addition to the effects already enumerated, 
appropriate methods of observation would show that vagus excitation increases the intraventricular pressure during diastole, lessens the intake and the output of the ventricle, and diminishes the tonus of the heart muscle. The action of the vagus is accompanied by a positive electrical variation. The action on the sinus and on the bulbus does not differ essentially from that upon the ventricle.

It has already been pointed out that the vagus of the frog contains both inhibitory and augmenting fibres. The stimulation of the mixed nerve usually causes inhibition, as described above, but sometimes augmentation. The augmentation observed after cessation of the inhibitory effect is probably explained by the longer after-effect of the augmentor excitation.

Intracardiac Inhibitory Mechanism. - Arrange an inductorium for tetanizing currents. Close the short-circuiting key. Expose a frog's heart. Raise the heart with a glass rod. Note the white "crescent" between the sinus venosus and the right auricle. Set the inductorium in action. Put the points of the electrodes on the crescent, and open the short-circuiting key for a moment. After one or two beats the heart will stop.

Inhibition by Stannius Ligature. - Turn up the heart to expose its posterior surface, and note the 
line of junction of the sinus venosus and right auricle. Tie a ligature around the heart exactly at this line, passing the thread beneath the aortre, so that they shall not be included in the ligature.

The auricles and ventricle cease to beat, for a time at least, while the sinus venosus continues with unaltered rhythm. (The result is usually ascribed to inhibition, from the mechanical stimulation of the intracardiac inhibitory mechanism. If the ventricle begins spontaneously to beat, as may happen if the ligature is not accurately placed, tie a second ligature around the junction of sinus and auricle.)

Action of Nicotine. - Apply nicotine solution (0.2 per cent) to the ventricle. After a few minutes, stimulate the trunk of the vagus nerve. No curve need be written.

The heart is not inhibited.

Now lift the heart with a glass rod, and stimulate the intracardiac inhibitory nerves.

The heart is inhibited. Nicotine paralyzes some inhibitory mechanism between the vagus and the intracardiac inhibitory nerves. But it is known that nicotine does not paralyze nerve trunks. Hence it is probable that the cardiac inhibitory fibres do not pass to the cardiac muscle directly, but end in contact with nerve cells, which take up the impulse and transmit it 
through their processes to the muscular fibres of the heart.

Atropine. - With a clean pipette apply a few drops of a solution of atropine ( 0.5 per cent) to the heart. After a few moments lift the ventricle and stimulate the crescent.

The heart is not inhibited. Atropine paralyzes the intracardiac inhibitory nerves.

Muscarine. - With a fine pipette put upon the ventricle a few drops of normal salt solution containing a trace of muscarine (a poisonous alkaloid extracted from certain mushrooms).

The ventricle will gradually be arrested in diastole, much distended with blood.

Antagonistic Action of Muscarine and Atropine. - With a fresh pipette apply a little normal salt solution of atropine ( 0.5 per cent).

The heart will commence to beat again.

\section{The Centres of the Heart Nerves}

It has been shown that the heart receives inhibitory and augmenting nerve fibres. The situation of the inhibitory and augmenting "centres," $i$. e., the nerve cells from which the inhibitory and augmenting fibres spring, should now be considered.

Inhibitory Centre. - Place a frog and a small sponge wet with ether under a glass jar. Be very 
careful not to kill the frog by an overdose of ether. When insensibility is complete, place the animal, back uppermost, on a frog-board. Cut through the skin in the median line from the nose about half way to the urostyle. Carefully uncover the roof of the skull. Remove the longitudinal muscles on either side of the 1 st, $2 d$, and $3 d$ vertebræ. Strip off the parietal bones with forceps, beginning at the anterior end, opposite the anterior margin of the orbit. Clear away the occipital bones. Saw through the laminæ of the first three vertebræ, and remove the laminæ to expose the spinal cord. Expose the heart by cutting away the chest wall over the pericardium. Hold the frog in such a way that the heart can be observed while the brain and cord are stimulated. With

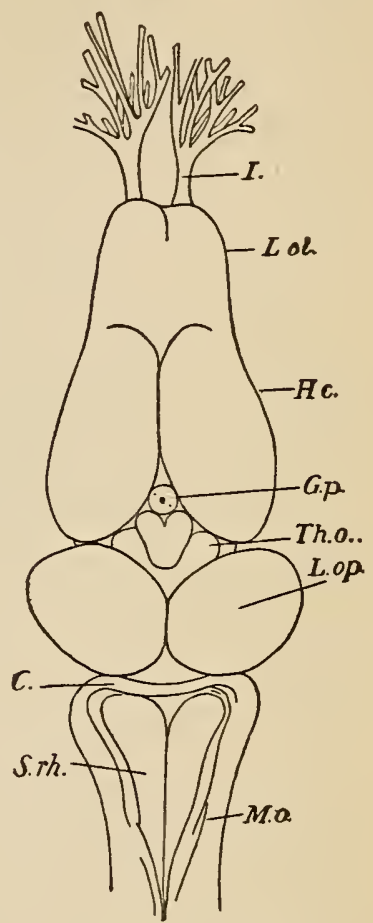

Fig. 74. View of the brain of a frog from above, enlarged. L.ol. Olfactory lobes. H.c. Cerebral hemisplieres. G.p. Pineal body. Th.o. Optic thalami. L.op. Optic lobes. C. Cerebellum. M.o. Medulla oblongata. S.rh. Sinus rhomboidalis. (After Foster's plate in BurdonSanderson's Handbook.) needle electrodes, the points of which should be 
one millimetre apart, stimulate the spinal cord with a tetanizing current of a strength easily borne on the tongue.

Stimulation of the spinal cord will not inhibit the heart. Stimulation of the cerebral hemispheres will be also ineffectual. Now stimulate the medulla oblongata. (Fig. 74.)

The heart will be inhibited.

This method of locating the cardio-inhibitory centre is unsatisfactory, because the inhibition produced may possibly be the result of the stimulation of nerve paths to or from the centre. Its results can be controlled by the method of successive sections, to be explained in connection with the vasomotor centre, page 565.

The cardio-inhibitory centre is always in action, for section of the vagi causes the heart to beat more frequently.

Augmentor Centre. - It is probable that this centre, like the inhibitory centre, is situated in the bulb, but the location is not definitely known. The constant activity of the augmentor centre is shown by the fall in frequency of beat after section of the vagi followed by bilateral extirpation of the inferior cervical and first thoracic ganglia in mammals.

The neuraxons, or axis-cylinder processes, of the augrnentor cells lying in the central nervous 
system pass out of the spinal cord in the white rami and terminate in the sympathetic ganglia (for example, the inferior cervical and stellate ganglia of the dog) in contact with sympathetic cells, the neuraxons of which convey the impulse to the heart.

The cardiac centres are readily affected by afferent impulses from many sources.

Reflex Inhibition of the Heart; Goltz's Experiment. - In a very lightly etherized frog, expose the pericardium by cutting away the chest wall over the heart. Count the number of beats in periods of twenty seconds. Continue the count while an assistant strikes gentle blows with the handle of a scalpel upon the abdomen at the rate of about 140 per minute.

The frequency will usually diminish and, in favorable cases, the heart will at length be arrested.

Cut both vagus nerves and repeat the experiment.

The reflex inhibition of the heart cannot be obtained after section of the vagi.

It has been shown by Bernstein that the afferent nerves in this experiment are abdominal branches of the sympathetic nerve. The stimulation of the central end of the abdominal sympathetic in the rabbit also produces reflex inhibition of the heart. 
Reflex Augmentation. - Count the human radial pulse during four consecutive periods of fifteen seconds. Let the subject sip cold water slowly. Repeat the count while the subject swallows.

The frequency will be increased.

Variations in the force and frequency of the heart-beat follow the stimulation of most afferent nerves, for example the central end of the divided vagus, the sciatic, and other mixed nerves, the nerves of special sense, and the afferent nerves which arise in the heart and pass to the bulb.

The most conspicuous of the nerves which bear impulses from the heart to the central nervous system in mammals is the depressor. This nerve occurs as an isolated trunk in the rabbit, and is found mixed with other fibres, for example in the vagus, in many other animals. The stimulation of the end of the severed depressor nerve in connection with the heart is without effect. The stimulation of the end in connection with the bulb slows the heart and dilates the blood-vessels, thus causing a great fall in the blood-pressure.

\section{Tire Innervation of the Blood-Vessels}

The Bulbar Centre. - 1. Lightly etherize a large frog. Expose and cut both vagus nerves (in order to exclude inhibition of the heart). It is of the first importance to avoid excessive hemor- 
rhage. Expose the brain and the anterior half of the spinal cord (page 565). Place the frog on the web-board. Note carefully the speed with which the corpuscles pass through the smaller vessels of the web. The rate of flow in the capillaries is the best practical index of the diameter of the small arteries. When the arteries constrict, the flow in the capillaries will be less rapid. Remove the cerebral hemispheres and the optic lobes. After five minutes or more (to allow the frog to recorer from the shock of the operation), note the condition of the web vessels.

There will be no significant change.

The removal of the brain anterior to the bulb has not destroyed the tonus of the blood-vessels.

Note the slow rhythmic changes in the diameter of the vessels. The changes are not uniform throughout the length of the blood-vessel.

2. Curarize the frog sufficiently to paralyze the motor nerves. Stimulate the bulb with very weak tetanizing currents.

The flow in the capillaries will be less rapid. Obviously the bulb contains nerve cells, the excitation of which causes the narrowing of the blood-vessels. These cells are termed the bulbar vasoconstrictor centre. Repeated sections show that the vasoconstrictor cells are placed (in the rabbit) on both sides of the median line from 
about one millimetre posterior to the corpora quadrigemina to a point about four millimetres posterior to those bodies.

The Vasomotor Functions of the Spinal Cord. 1. Divice the cord just posterior to the bulb. (A fresh frog may be required. In that case, remember to curarize.)

The division of the fibres connecting the vasoconstrictor centre with the cord will be followed by the dilatation of the vessels in the web (i.e. the flow will be more rapid).

2. Stimulate the peripheral segment of the divided cord.

The blood-vessels will constrict.

Thus the neuraxons (axis-cylinder processes) of the bulbar vasomotor cells pass through the spinal cord on the way to their respective bloodvessels.

It should now be determined whether these fibres pass to the blood-vessels without interruption, or whether they eud in contact with spinal vasomotor cells through which the connection with the blood-vessels is made.

3. Wait five minutes and then note the flow through the capillaries.

The dilatation observed immediately after the separation of the cord from the medulla has given place to moderate constriction. 'The tonus of the 


\section{INNERVATION OF HEART AND BLOOD-VESSELS 571}

blood-vessels has returned. The spinal cord has taken up the vasomotor function of the bulb. Evidently the spinal cord contains vasomotor cells, which ordinarily are subsidiary to those of the bulb, but which, when separated from their master cells, acquire the power of independent action.

Effect of Destruction of the Spinal Cord on the Distribution of the Blood. - Further evidence of the vasomotor function of the spinal cord is afforded by the following experiment.

Expose the heart, avoiding unnecessary loss of blooci. Lay bare the upper part of the intestine by an incision on the left side of the umbilical vein, which lies in the median line. Suspend the frog vertically. Note that the heart and the great vessels are filled with blood. Note also the size and number of the vessels in the walls of the stomach and intestines.

Bend the frog's head. Put the seeker into the vertebral canal and pass it gently downwards to destroy the spinal cord. The seeker will move easily, if really in the canal. Look at the heart and great arteries.

The heart will soon be bloodless, though beating regularly. Examine the vessels of the stomach and intestine. They are distended. Evidently, the contents of the heart and the great arteries 
have passed into dilated smaller arteries and veins. It would be found, on waiting, that this effect is not a passing consequence of inhibition. The destruction of the spinal cord has changed the distribution of the blood.

The Vasomotor Fibres leave the Cord in the Anterior Roots of Spinal Nerves. - 1. Remove the arches of the 5th, 6 th, 7 th, 8 th, and 9 th vertebre and lay bare the cord in a large frog in which the motor nerves have been paralyzed with curare. Note the capillary flow in the web. On the side on which the web-vessels are examined, tie a silk thread around each of the anterior roots near their origin from the cord, and sever the roots between the ligature and the cord.

The vessels will dilate.

2. Stimulate the peripheral ends of several of the divided roots.

Constriction will follow.

The vascular dilatation which follows the destruction of the spinal cord is not permanent. After a time the vessels regain their tonus. It is probable, therefore, that vasomotor nerve cells exist outside the spinal cord, and this conclusion is confirmed by the results gained on warm-blooded animals with the nicotine method. Langley has found that the injection of about ten milligrams of nicotine into a vein of a cat will prevent, for a 
time, the passage of nerve impulses through sympathetic cells. Painting the ganglia with nicotine has the same effect. In animals the sympathetic cells of which have thus been paralyzed, the stimulation of the lumbar nerves in the spinal canal produces no change in the vessels of the generative organs, though in animals not poisoned with nicotine this stimulation causes marked constriction. The lumbar vasomotor fibres must therefore end in connection with sympathetic nerve cells which transmit the constrictor impulse to the blood-vessel. Similar observations in other regions warrant the belief that all the vasomotor fibres emerging from the spinal cord end in like manner.

Thus the vasoconstrictor system probably consists of three neurons. The first is a sympathetic cell, lying apart from the central nervous system. Its neuraxon (axis-cylinder process) passes directly to the blood-vessel. The second is a spinal cell, the neuraxon of which leaves the cord and terminates in contact with the sympathetic cell or its branches. The third has its cell body in the bulb and its neuraxon terminates in contact with the second neuron.

Commonly, as for example in the nerves of the extremities, the sympathetic neuraxon passes from the ganglion along the gray ramus into the 
corresponding spinal nerve, in which it continues to its distribution.

Vasoconstrictor Fibres in the Sciatic Nerve. Curarize a frog sufficiently to paralyze the voluntary muscles (any excess of curare will paralyze the vasomotor fibres also). Carefully destroy the brain with the seeker, avoiding loss of blood. Expose the right sciatic nerve for a short distance on one side, using the greatest care not to injure the blood-vessels. Tie a thread tightly around the nerve near the upper end of the exposed portion. Lay the frog, back upward, on the web-board, placing the web of the right foot over the notch, and securing it with fine pins. Examine the web under a low power, to make sure that the circulation has not been interrupted by stretching the web. Place the secondary at such a distance from the primary coil that the induced current shall be barely perceptible to the tongue. Set the hammer vibrating, and close the short-circuiting key. Put the electrodes under the sciatic nerve on the peripheral side of the ligature. Let a second observer watch a sinall vessel of the web through the microscope. Open the short-circuiting key for a moment only.

The blood-stream slows from constriction of the supplying vessels, the contraction increasing during a few seconds and then subsiding. 
This experiment requires much care and close observation. The curare effect must be very slight; a small quantity of the drug should be given an hour before the observation is made. Great pains must be taken to use feeble currents and not to prolong the excitation, for the vasomotor nerves are rapidly exhausted. The narrowing of the arteries of the web is usually evident only in the slowing of the blood-stream during excitation.

Vasodilator Nerves. - 1 . Repeat the preceding experiment in a frog in which the sciatic nerve has been four days severed (without injury to the femoral vessels). On stimulation of the peripheral segment of the divided sciatic nerve, the ressels of the web will dilate instead of constricting.

Evidently the sciatic nerve contains vasodilator as well as vasoconstrictor fibres. When the sciatic fibres are separated from their cells of origin by the section of the nerve, the fibres distal to the section degenerate. But the degeneration does not proceed at the same rate in all the fibres. The vasoconstrictors die before the vasodilators. In ordinary stimulation of the normal nerve, the action of the constrictors overpowers that of the dilators. In the partially degenerated nerve, the same stimulation causes dilatation because the constrictor fibres are dead or dying. 
2. Note the rate of flow in the web-vessels in the uninjured limb. Stimulate the sciatic nerve with the single induction current repeated at intervals of five seconds.

The vessels of the web will dilate.

The vasoconstrictor and vasodilator fibres also react differently to cold. If the lind $\operatorname{limb}$ (cat) be cooled, the stimulation that normally causes vasoconstriction will cause vasodilatation.

Vasoconstrictor and vasodilator fibres are not always found in the same nerve-trunks; in the chorda tympani nerve, for example, there are only dilator fibres.

The central relations of the dilator nerves have not been sufficiently studied to warrant their discussion here.

Reflex Vasomotor Actions. - 1. Note the rate of flow in the vessels of the web in a lightly curarized frog. Stimulate the skin (not too near the bulb or cord) with tetanizing currents. The stimulus must not be repeated often, or fatigue will obscure the result.

Reflex constriction of the vessels will take place. The sensory inmpulse is carried by afferent fibres to the vasomotor centres.

Repeat the experiment, using in place of the electrical a mechanical stimulus, such as pinching the skin with forceps. 


\section{Apparatus}

Normal saline. Bowl. Towel. Pipette. Glass plate. Inductorium. Key. Wires. Dry cell. Electrodes. Needle electrodes. Frog-board. Electromagnetic signal. Heart-holder. Kymograph. Glass tube for oesophagus. Two muscle levers. Solutions of nicotine ( 0.2 per cent), atropine ( 0.5 per cent), muscarine (a trace in normal salt solution). Curare. Ether. Sponge. Glass jar. Vertebral saw. Web-board. Fine pins. Microscope. Frog, the sciatic nerve of which has been severed four days. Millimetre rule. Silk thread. 



\section{N D E X}

Aberration, chromatic, 432, 434; diaphragm, 434; spherical, by reflection, 426 ; spherical, by refraction, 427,434 .

Absolute force of muscle, 358 .

Accommodation, 469; angle between light and visual axis, 487 ; far point, 479 ; iris, 474 ; lens, $474,475,477$; line, 472 ; measurements, 479 ; mechanism, 473 ; pupil, 473 ; pupil, nearness, 488 ; pupil, size, 488 ; range, 471,484 .

Acuteness of vision, 465, 466 .

Action current, brain and cord, 319 ; decrement, 309 ; duration, 314; glands, 320 ; heart, 310, 312; threshold value, 318; human muscle, 309 ; muscle, 300 ; nerve, 315 ; optic nerre, 318 ; positive after current, 317 ; positive variation, 316 ; precedes change in form, 211 ; tetanus, 305 ; voltage, 315.

Afferent impulses, reflex action, 371 ; summation, 372 .

Alteration hypothesis of nerve and muscle current, 299.

Amalgamation, 46

Ametropia, determination of, 493.

Angle, construction of tangent, 467 ; incidence, 403 ; reflection, 403 ; refraction, 411 ; sine, 414 ; visual, 464 .

Angle gamma, 464 .

Animal heat, 285.

Ankle jerk, 376 .

Anodes and cathodes, physiological, 110.

Anterior roots, vasomotor fibres, 572 .

Aortic regurgitation, 550.

Aortic stenosis, 551 .

Aperture, 420, 427.

Apparatus, criticism of, 84 .

Arrhenius, theory of dissociation, 31 .

Artificial scheme, 511 .

Astigmatism, 464 ; measurement, 495.

Atropine, action on heart, 564 .

Angmentor centre, 566.

Axis, optical, 419; optical, eye, 439 ; principal, 419 ; visual 463. 
BaLANCING experiment, 379.

Bernstein's experiment, 531 ; rheotome, 313.

Blood pressure, arteriai in frog, 522; influenced by inhibition, 525 ; peripheral resistauce, 523.

Blood-ressels, innervation, 568.

Brain of frog, 565.

Brain, destruction by pithing, 97; dorsal view, 293.

Calcrum, in normal solution, 165 .

Calorimeter, Rubuer's experiment, 285.

Carbon dioxide, action on nerve, 172; apparatus, 173.

Caustic surface, $427-429$.

Cell, dry, 52: Daniell, 48; galvanic, 34.

Cell, in series, 133.

Centre, rotation, 463 ; optical, 420 ; optical, crystalline lens, 446 .

Centres of heart nerves, 564 .

Cerebral hemispheres, removal of, 378.

Chemical stimulation, 163.

Circle, dispersion, 429, 470-472.

Circulation, artificial scheme, 511 ; capillary, 262, 569 ; intermittent and continnous, 515 ; mechanics of, 508 ; mesentery, 519 ; rate of flow and width of bed, 519.

Clamp, double, 65 ; flat-jawed, 65 ; Gaskell, 103 ; round-jawed, 65.

Clausius, theory of dissociation, 29.

Closing contraction, 98.

Color blindness, 501 .

Compensation of demarcation current, 294.

Compensatory pause, 533.

Conductivity, 168; centripetal and centrifugal, 181; during constant current, 123.

Contraction, tonic, 141 .

Contraction, direction of current, 157 ; human muscle, 353; idiomuscular, 166; law, 113 ; load, 341 ; opening and clesing, 98 ; rhythms, 142 ; single, 332 ; temperature, 342 ; tonin., 107 , 140 ; veratrine, 345 ; wave, 338 ; heart muscle, 534.

Contracture, 340 .

Coördinated actions, 378 .

Croak reflex, 379.

Curare, 97 ; poisons end plates, 171.

Danieti, cell, 48.

I)ecrement of action current, 309 . '

Demarcation current, 287, 295; hypotheses, 297 ; interferes with stimulatiug current, 292 ; measurement, 292; muscle, 287 ; negative variation, 305 ; nerve, 296 ; stimulus, 289, 296.

Deunett's incthod, numbering prisms, 435. 
Depressor nerve, 568.

Deviation, angular, 435 .

Dicrotic notch, 545 .

Diffusion of gases, 14.

Dioptre, 435.

Dispersion circle, $429,470,471,472$.

Distance, focal, crystalline lens, 450 ; principal focal, cornea, 441 .

Distilled water, a chemical stimulus, 163.

Drying, l49, 164.

DuBois-Reymond, molecular theory, 298.

Duchenne's points, 127.

Duration of stimulus, 138.

ELASTICITy and extensibility, of a metal spring, 364 ; of a rubber band, 364 ; of skeletal muscle, 365 .

Electric fish, 329.

Electrical units, 35 .

Electrodes, for human nerves, 132 ; indifferent, 111 ; non-polarizable, 93 ; platinum, 65.

Electrolysis, 26.

Electrolytic solution pressure, 32.

Electrometer, 34.

Electromotive force, 34, 287 ; demarcation current, 292.

Electrotonic currents, 323 ; as stimulus, 328 ; negative and positive variation, 325 ; polarization increment, 325 .

Energy, set free in various forms, 10 ; stimulation, and irritability, 7 ; developing, 361 .

Emmetropia, 490; augle of, ${ }^{\prime} 464$.

Engelmann's incisions, 534 .

Ergograph, 354.

Excitation wave, 336 ; remains in original fibre, 181.

Extensibility, 364, 366 .

Extra contraction of heart, 533.

Eye, artificial, ophthalmoscopic, 489 ; as camera obscura, 437 ; normal measurements, 461 ; see optical box, 404 ; reduced, 458 ; schematic, 438.

Extra current in indnctorium, 68.

Fatigue, 367 ; human muscle, 368 ; polar, 147.

Fixation, line, 463.

Focus, conjugate, concave mirror, 427 ; conjugate, convex lens, 418 ; conjugate, cornea, 444 ; principal, concave mirror, 405; principal, construction, 443; principal, convex lens, 416; principal, eye, 454.

Focal distance, concave mirror, 406 ; principal, convex lens, 417. Focal line, 427. 
Food materials, composition of, 281.

Flexors and extensors, relative excitability, 177.

Frog board, 112.

Galvaxic cells, electromotive force in, 34 .

Galvanic stimulation may cause periodic impulses, 144 .

Galvanotropism, 137.

Gas chamber, 173.

Gaskell's block, 535; clamp, 103.

Goltz's experiment, 567.

Gower's experiment, 376.

Graphic method, 77.

HeART, action current, 310, 312 ; apex, isolated, 531 ; atropine, 564 ; augmentor centre, 566 ; augmentor nerves, 555 ; auriculo-ventricular interval, 561 ; Bernstein's experiment, 531 ; calcium, 539; change in form, 529; chemical theory, 540; compensatory pause, 533 ; constant stimulus, 532 ; contraction curve, 530 ; contraction wave, 534 ; excitation from auricle to ventricle, 535; exposure, 112; extra contraction, 533; Gaskell's block, 535; graphic record, 530; impulse, 541 ; inhibited, 559 ; inhibitory centre, 564 ; inhibitory mechanism, 562 ; inorganic salts, 538 ; irregularities, 536 ; irritable though inactive, 533 ; irritable though inhibited, 561 ; load, 537 ; maximal contraction, 530 ; monopolar stimulation, 111 ; muscarine, 564; nerve-free, 170 ; nicotine, 563 ; outflow period, 526; polar inhibition, 153; polar stimulation, 110; potassium, 539 ; pump, 525 ; reflex augmentation, 568 ; reflex inhibition, 567 ; refractory period, 533 ; rhythmic contractility, 532 ; sounds, 541 ; staircase contraction, 531 ; tonus, 537 ; Stannius inhibition, 562 ; sympathetic, 556 ; temperature, 538 ; vagus, 559 ; valves, $511,525,550,551,552$.

Heat values, calculation, 285.

HYpermetropis, 431 ; angle of, 464; measurement, 495.

InIO-MUSCULAR contraction, 166.

Image, concave milror, 405, 409; convex mirror, 410; convex lens, 419,420 ; cornea, 443 ; dioptric, 456,457 ; retinal, 437 ; retinal, actual size, 465 ; retinal, apparent size, 464 ; smallest perceptible, 466 ; virtual, concave mirror, 408 ; virtual, concave lens, 419.

Index of refraction, 412 .

Induction currents, 54 ; direction, 158 ; gap in resulting contraetions, 161 : maguetic, 56, 58 ; nerves, 69 ; stimulns, 66,158 ; unipolar, 71 .

Inductorium, 54 ; constrnction, 60 ; graduation, 83. 
Inhibition, galvanic, 153; heart, 559 ; polar, 155 ; reflex of heart, 567 ; ventricular, 524 .

Inhibitory nerves of heart, 558 .

Inhibitory centre, 564 .

Interrupter, 62, 303.

Ions, 28.

Iris, accommodation, 474 .

Irritability, 169 ; definition, 9 ; different points of same nerve, 180 ; flexor and extensor nerves, 177 ; muscle, independent, 169 ; nerve greater than muscle, 179 ; separable from conductivity, 172.

Isometric contraction, 352, 355 ; method, 349.

Isotonic method, 349 .

Isotony, 20.

KEY, short-circuiting, 46 ; simple, 45; rocking, 50.

Kinetic theory, 12.

Knee jerk, 375.

Kymograph, 79; long paper, 81.

LANTERN, 404.

Latent period of muscle, 334 .

Lens, accommodation, 474, 475 ; concave, 422 ; convex, 416 ; numbering, 435.

Lever, light muscle, 86 ; heavy or rigid, 351 ; writing, 87.

Light, spectrum, 413.

Line of fixation, 463 ; focal, 427 ; force, 57.

Load, influence on contraction, 537.

Magnetic field, 57 ; induction, 57.

Make or break current excluded, 70 ; stimuli, 67.

Manometer, mercury, 523.

Mechanical stimulation, 166 .

Mirror, concave, 405 ; convex, 410 ; plane, 403.

Mitral incompetence, 552 .

Moist chamber, 95 .

Molecular hypothesis of nerve and muscle current, 298.

Monopolar stimulation, 111.

Motor points, 128.

Muscarine, action on heart, 564 .

Muscle, action current, 300 ; clamp, 8 ; curre, 333 ; demarcation current, 287; form affects stimulation, 156; left hind limb of frog, 6, 99; lever, 86,351 ; tonus, 389 ; turbid and clear, 335 ; warmer, 343.

Myomeres, 298.

Myopia, 430 ; angle of, 464 ; measurement, 493. 
Negative variation, 321 ; electrotonic currents, 325 ; secretion currents, 321.

Nerve, action current, 315 ; cervical in frog, 558 ; conducts in both directions, 181 ; condnctivity, 120 ; conductivity and irritability, 172; demarcation current, 295; drying, 149; electrical resistance, 327 ; electromotive phenomena, 295; impulse, speed of, 184 ; induction, 69 ; inhibitory, 558 ; irritability, 116; irritability compared with muscle, 179; irritability, different points, 180 ; irritability, specific, 179 ; polarization, 323 ; polar stimulation, 113,131 ; stimulated by own demarcation current, 296.

Nerve-muscle preparation, 4.

Nicotiue, action on heart, 563 .

Nitrite of amyl, 550.

Normal saline solution, 165 .

OpHTHALMOSCOPE, 489.

Ophthalmoscopy, 484; direct, 490 ; indirect, 496.

Optic nerve, action current, 318.

Optical box, 404 .

Opening and closing contraction, 98, 125 ; tetanus, 147.

Ordinates, 91.

Osmometer, 18.

Osmotic pressure, 16; blood-corpuscle method, 22; blood serum, 21.

PAPER, smoked, method of using, 78.

Paradoxical contraction, 328.

Paramecium, galvanotropism, 137.

Partial pressure, 13.

Periodic contraction from chemical stimulation, 165 ; galvanic stimulation, $1+4$.

Peripheral resistance, 521.

Permeability, 24.

Pithing, 97.

Plasmolysis, 20.

Plethysinograph, 545.

Point, cardinal, cornea, 440; cardinal, crystalline lens, 445; cardinal, eye, 4.39, 451 ; far, accommodation, 479; far, determination, 479 ; near, accommodation, 480 ; near, determination, 480 ; nodal, 420 ; nodal, crystalline lens, 447 ; nodal, eye, 453 ; principal, crystalline lens, $450 ; s, 449,453$.

Polar excitation, 148 ; fatigue, 147 ; inhibition, 153,155 ; injured muscle, 151 ; refusal, 292; stimulation, 101, 113, 159.

Polarization, 46; current, 51, 145; increment, 325; positive variation, 146 .

Pole-changer, 49, 50. 
Positive after current, 317.

Yositive variation, action current, 316 ; polarization current, 146 ; polarizing current, 325 .

Prentice's method, numbering prisms, 435.

Prisms, 413; construction, 413; numbering, 435 ; path of entering ray, 413.

Pnlse, aortic regurgitation, 550; curve, 546; dicrotic, 545; form, 544; frequency, 543 ; hardness, 544 ; low tension, 549 ; pressure, 545 ; valvular disorders, 551 , 552; volume, 545, 552.

Pupil, accommodation, 473.

REACTION of degeneration, 135.

Reaction time, 382 .

Reflection, concave mirror, 405; convex mirror, 410; plane mirror, 403.

Reflex actions, 370 ; afferent impulses, 371 ; cornea, 374 ; iuhibition, 384 ; man, 374 ; pupil, 375 ; purpose, 381 ; segmental, 373; strychnine, 377; tendon, 375 ; threshold, 372; throat, 382 ; vasomotor, 576 .

Reflex time, 382 .

Refraction, 410 ; concave lens, 422 ; convex lens, 416 ; convex and cylindrical lenses, combined, 424 ; cylinders, 422 ; eye, 437 ; index, 412 ; prism, 413.

Refractory period, 534 .

Respiration, mechanics of, 505.

Respiration scheme, 505.

Retina, reflection, 484 .

Rheochord, 42.

Rheoscopic frog, 302, 306.

Rheotachygraph, Hermann, 314.

Rheotome, differential, Bernstein, 313.

Ringer solution, $5 \star 0$.

Ritter-Rollett phenomenon, 177.

SALTS, influence on contraction of heart, 538 .

Saturation, 16.

Scheiner's experiment, 469.

Sciatic nerve, vasomotor fibres, 574 .

Secretion current, 320 ; negative variation, 321.

Semi-permeable membrane, 17 .

Shortening in single contraction and in tetanus, 348 .

Sensation, effort, 400 ; general, 398 ; irradiation, 398 ; motion, 400 ; motor, 400 ; pain, 399 ; pressure, 393 ; taste, 401 ; temperature, 390 ; tickle, 398 ; touch, 395 ; Weber's law, 395.

Signal, electro-magnet, 105.

Size, apparent, $456,465$. 
Skin, hot and cold spots, 390 ; irradiation, 398 ; pressure spots, 393.

Smooth muscle, 356 .

Solution, gas in liquid, 16; normal, 165 ; solid in liquid, 16 ; teusion, 16.

Spectrum, 413, 432 .

Sphygmograph, 527.

Spinal cord, localization of movements at different levels, 387 ; destruction changes distribution of blood, 571 .

Spinal nerve roots, 386 ; sensory nerves, 388 .

Spontaneous contractions, 356.

Staircase contraction, heart, 531 .

Stannius ligature, 562.

Stimulation, 9 ; angle of curreut lines, 157 ; chemical, 163, 164 ; constant, may cause periodic contraction, 165; demarcation current, 290, 296; distilled water, 163; drying, 164; form of muscle, 10, 156; induction current, 158 ; intensity changes, 99 ; mechanical, 166; minimal and maximal, 175; monopolar, 111; polar, in heart, 110 ; summation of impulses, 176 ; threshold value, 174 ; unipolar, errors, 320.

Stroboscopic method, 305 .

Surface, caustic, 427, 428, 429; principal, crystalline lens, 418 ; principal, eye, 449.

Surface tension, 25,36 .

Summation of stimuli, 176 .

Superposition in tetauus, 347 .

Superposition of two contractions, 346 .

Sympathetic, action on heart, 556; frog, 556; preparation, 555.

Synchronous points, method of obtaining, 120.

System A, schematic eve, 440; B, schematic eye, 445; C, schematic eye, 451 .

Temierature, hourly variation, 285 ; mouth, affected by food, 285 ; reaction to variatious in, 285 ; regional, 285.

Tension indicator, 25.

'Tetanus, 69, 346 ; electrical phenomena, 305 ; natural and artificial, 355 ; opening and closing, 147 ; Ritter's, 149.

Threshold value of stimulation, 175 .

Tonus of heart muscle, 537.

Tradescantia discolor, 20.

Tuning fork, 88.

UNIPOLAR induction, 7l, stimulation, errors, 320.

VAN ILER WAAL's hypothesis, 14.

Van't Hoff's discoveries, 20. 
Vagus, preparation, 558; inhibits heart-beat, etc., 559, 560, 561 .

Vapor pressure, 15.

Vasodilator nerves, 575 .

Vasomotor centre, 570 ; fibres in anterior roots, 572 ; functions of cord, 570; reflexes, 576 ; sciatic, 574 .

Veratrine, influence on contraction, 345 .

Vision, acuteness, 465, 466; blind spot, 499; color blindness, 501 ; field of, 500 ; yellow spot, 499.

Volume of contracting muscle, 331.

Volume tube, 332.

WÖHLER'S discovery, 3.

Work adder, 359.

Work done, influenced by load, 358 . 





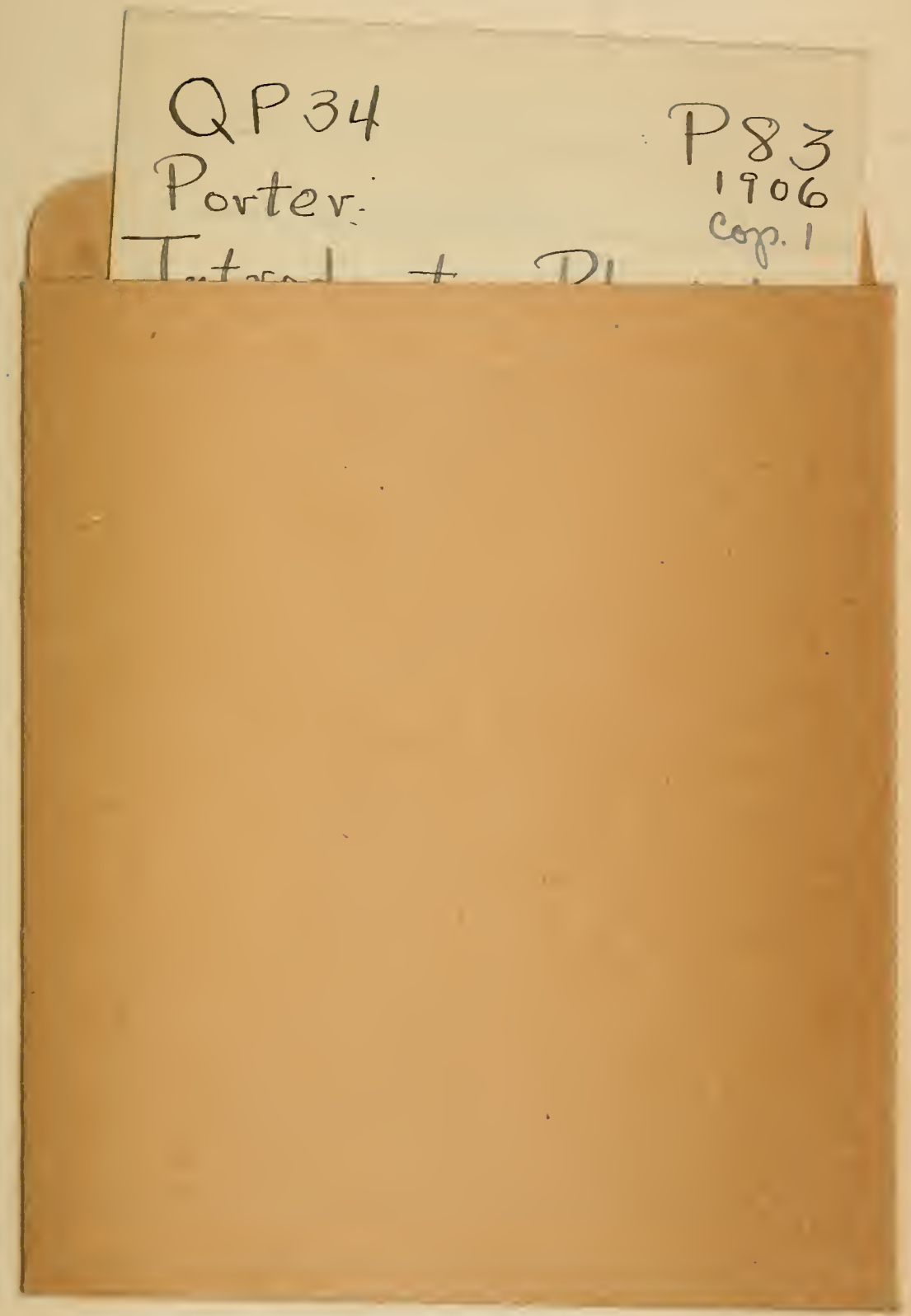

JUN 261928 
